\title{
Boundization, Closure and Convergence For The Ultra Extended Complex Banach and Hilbert Spaces with Applications
}

\author{
Salah Hamad \\ WVU, shamad@mix.wvu.edu
}

Follow this and additional works at: https://researchrepository.wvu.edu/etd

Part of the Other Applied Mathematics Commons

\section{Recommended Citation}

Hamad, Salah, "Boundization, Closure and Convergence For The Ultra Extended Complex Banach and Hilbert Spaces with Applications" (2018). Graduate Theses, Dissertations, and Problem Reports. 3709. https://researchrepository.wvu.edu/etd/3709

This Dissertation is protected by copyright and/or related rights. It has been brought to you by the The Research Repository @ WVU with permission from the rights-holder(s). You are free to use this Dissertation in any way that is permitted by the copyright and related rights legislation that applies to your use. For other uses you must obtain permission from the rights-holder(s) directly, unless additional rights are indicated by a Creative Commons license in the record and/ or on the work itself. This Dissertation has been accepted for inclusion in WVU Graduate Theses, Dissertations, and Problem Reports collection by an authorized administrator of The Research Repository @ WVU.

For more information, please contact researchrepository@mail.wvu.edu. 


\title{
Boundization, Closure and Convergence For The Ultra Extended Complex Banach and Hilbert Spaces with Applications
}

\author{
Salah Hamad \\ Dissertation submitted to the \\ Eberly College of Arts And Sciences \\ at West Virginia University \\ in partial fulllment of the requirements \\ for the degree of \\ Doctor Of Philosophy \\ in \\ Mathematics \\ Harry Gingold, Ph.D., Chair \\ Leonardo Golubovic, Ph.D. \\ Harumi Hattori, Ph.D. \\ Dening Li, Ph.D. \\ Jocelyn Quaintance, Ph.D. \\ Jerzy Wojciechowski, Ph.D. \\ Department of Mathematics \\ Morgantown, West Virginia \\ 2018
}

Keywords:Boundization,The Ultra Extended Banach and Hilbert Spaces

Copyright 2018 Salah Hamad 


\begin{abstract}
Boundization, Closure and Convergence For The Ultra Extended Complex Banach and Hilbert Spaces with Applications

Salah Hamad

We study Boundization, Closure and Convergence of Complex Banach and Hilbert spaces. We augment a Banach space with elements "infinity" and turn the augmented set into a metric space by appropriate distance functions. This new metric space is called the Ultra Extended Banach Space. A new family of bijections, motivated by nonlinear projections, take an Ultra Extended Banach Space into bounded subsets of a "larger" Banach space. We compare the family of new metrics induced and show which ones are equivalent and which ones are unexpectedly not equivalent. Some applications are provided.
\end{abstract}




\section{Acknowledgments}

I would like to express my deepest appreciation to my committee chair and advisor, Dr.Gingold, Harry, for giving me the opportunity to work with him. This dissertation would not be possible without his constant guidance and support. Dr. Gingold, constantly supervised my work and gave a lot of suggestions regarding my research. A special gratitude I give to Dr. Golubovic, Leonardo, Dr. Hattori, Harumi, Dr. Li, Dening, Dr. Quaintance, Jocelyn, and Dr. Wojciechowski, Jerzy, for being on my committee. I have been very fortunate to have had the opportunity to take courses with some of my committee members, and their teaching has been essential to my understanding of the subject. I would also like to thank the Department of Mathematics of West Virginia University for its support regarding the results obtained in this thesis. Furthermore I would also like to acknowledge with much appreciation professors Dr. Harvey Diamond, who have been a great help to me over the last few years. I would like to acknowledge my family who supported me during my time here. Last but not least, I wish to thank everybody with whom I had stimulating discussions that led to the inception of this thesis, and their suggestions for indicating ways that led to clarify the presentation, concepts and results. Finally, I would like to express my gratitude to my family. First to my wife, Fatma, for help me to regain hope after despair, resume life after obstructions, restart journeys after detours, revive strength after defeat and resurrect dreams after rejection. Thank you for keeping me going through all the stresses. I would be remiss if I did not also express my thanks to my parents, brothers and sisters for giving me the motivation to pursue higher studies and gave me lot of valuable suggestions which are helpful during my stay here in US. I want to say thanks to all of my friends at WVU, who stayed with me all the time and made my stay at WVU so memorable. Their support seemingly has no limit, and has been much appreciated throughout my life. I want to dedicate this thesis to parents of my wife Youssuf and Jamila, my son Malik, and my daughters Sandy and Sally. 


\section{List of Figures}

3.1 Sphere . . . . . . . . . . . . . . . . . 22

3.2 Paraboloid . . . . . . . . . . . . . . . . . . . . . . . . 29

3.3 Cone . . . . . . . . . . . . . . . . . . . . . 38

3.4 Hyparaboloid . . . . . . . . . . . . . . . . . . . 43

3.5 Ellipsoid . . . . . . . . . . . . . . . 51 


\section{Contents}

1 Introduction 1

1.1 Overview and History . . . . . . . . . . . . . . . . 1

2 The Ultra Extended Banach and Hilbert Spaces 7

2.1 The Ultra Extended Banach Space . . . . . . . . . . . . . 7

2.2 The Ultra Extended Hilbert Space . . . . . . . . . . . . . . . 9

2.3 Operations in the Ultra Extended Banach and Hilbert Spaces 11

2.4 Convergence in the Ultra Extended Banach and Hilbert Spaces 13

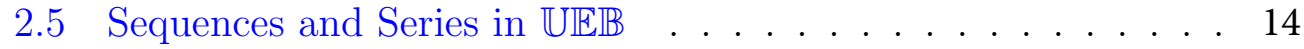

3 Boundization and Closure 16

3.1 What are Normalized Boundizations? . . . . . . . . . . . . 16

3.2 Closure . . . . . . . . . . . . . . . . . . . 17

3.3 Banach Spaces versus Hilbert Spaces . . . . . . . . . . . . . . 18

3.4 A General Surface Boundization and Geometrical Considerations ...................... . . 19

3.5 A Sphere Boundization . . . . . . . . . . . . 21

3.6 A Paraboloid Boundization . . . . . . . . . . . . . 29

3.7 A Cone Boundization . . . . . . . . . . . . . . . . 37

3.8 A Hyperboloid Boundization . . . . . . . . . . . . . . . 42

3.9 An Ellipsoid Boundization . . . . . . . . . . . . . . 51

4 A General Metric Induced 59

4.1 General Theorems . . . . . . . . . . . . . . . 59

4.2 Approximating Schemes in Banach spaces via Boundizations . 64

4.2.1 Consistency of approximations in Banach Spaces . . . . 65

4.2.2 Convergence in A General Surface Boundization . . . . 66

4.2.3 Examples of A General Surface Boundization . . . . . 69 
4.3 On A Surface of A Sphere . . . . . . . . . . . . . 70

4.3.1 The Sphere Metric Induced . . . . . . . . . . . . 71

4.4 On A Surface of A Paraboloid . . . . . . . . . . . . . . . 74

4.4.1 The Paraboloid Metric Induced . . . . . . . . . . . 75

4.5 On A Surface of A Cone . . . . . . . . . . . . . 78

4.5.1 The Cone Metric Induced . . . . . . . . . . . . 78

4.6 On A Surface of A Hyperboloid . . . . . . . . . . . . . 80

4.6.1 The Hyperboloid Metric Induced . . . . . . . . . . . 81

4.7 On a Surface of An Ellipsoid _ . . . . . . . . . . . . . 83

4.7.1 The Ellipsoid Metric Induced . . . . . . . . . . . . 83

5 Equivalence and Nonequivalence Between Extended Metrics 87

5.1 Metrics Nonequivalence and Metrics Equivalence . . . . . . 87

5.2 General Theorems for Metrics . . . . . . . . . . . . . 88

5.3 Equivalence Between An Extended Cone Metric and An Extended Paraboloid metric . . . . . . . . . . . . . . . . . 98

5.4 Equivalence Between Two Extended Cone Metrics . . . . . . . 103

5.5 Equivalence Between An Extended Cone Metric and An Extended Hyperboloid Metric . . . . . . . . . . . . . 106

5.6 Equivalence Between An Extended Sphere Metric and An Extended Ellipsoid Metric when $\alpha_{s} \neq 0$ and $\alpha_{e} \neq 0 \ldots \ldots . \quad 111$

5.7 Equivalence Between An Extended Sphere Metric with $\alpha_{s}=0$ and An Extended Ellipsoid Metric with $\alpha_{e}=0 \ldots \ldots . \quad 116$

5.8 Equivalence Between Two Extended Sphere Metrics when $\alpha_{s} \neq 0 \ldots \ldots \ldots \ldots \ldots \ldots \ldots \ldots \ldots \ldots$

5.9 Equivalence Between Two Extended Sphere Metrics when $\alpha_{s}=$ 0 . . . . . . . . . . . . . . . . . . . . . 129

5.10 Equivalence Between Extended Sphere Metric when $\alpha_{s} \neq 0$ And An Extended Cone Metric . . . . . . . . . . . . . . . 133

5.11 Nonequivalence Between An Extended Sphere Metric when $\alpha_{s}=0$ and An Extended Cone Metric ............ 138

6 Equivalence and Nonequivalence Between An Extended Metric and A Projection Metric 139

6.1 General Theorems . . . . . . . . . . . . . . . . . . . . . . 139

6.2 Equivalence Among Any Extended and Any Projection Cone Metric, Any Paraboloid Metric and Any Hyperboloid Metric. 141 
6.3 Nonequivalence Between A Projection Sphere Metric with $\alpha_{s} \neq$ 0 and A Projection Cone Metric . . . . . . . . . . . . . . . . 142

6.4 Nonequivalence Between A Projection Sphere Metric with $\alpha_{s}=$ 0 and A Projection Ellipsoid Metric with $\alpha_{e}=0 \ldots \ldots$.

6.5 Nonequivalence Between A Projection Sphere Metric with $\alpha_{s}=$ 0 and A Projection Cone Metric . . . . . . . . . . . . . . . 144

6.6 Nonequivalence Between A Projection Ellipsoid Metric with $\alpha_{e}=0$ and A Projection Cone Metric . . . . . . . . . 145

6.7 Nonequivalence Between The Projection Sphere Metrics with $\alpha_{s} \neq 0$ and The Projection Sphere Metrics with $\alpha_{s}=0 \ldots 14$

6.8 The Projection of Ellipsoid Metrics is Not Equivalent to The Extended Ellipsoid Metrics $\alpha_{e} \neq 0 \ldots \ldots \ldots \ldots \ldots$.

6.9 The Projection Sphere Metric with $\alpha_{s}=0$ is Not Equivalent to Its Extended Metric . . . . . . . . . . . . . . . . . . 151

6.10 The projection Ellipsoid Metric with $\alpha_{e}=0$ is Not Equivalent to Its Extended Metric . . . . . . . . . . . . . . . . . 152

7 Conformal Mapping and Triangles Similarity in Sphere Boundization

7.1 The Angle Measure Between The Images of Two Curves . . . 156

7.2 Similarity of Triangles . . . . . . . . . . . . . . . 171

7.3 Reformulation of The Main Theorem and Its Proof . . . . . . 173

7.4 Examples and Utility of The Multi Parameter Family . . . . . 174

7.5 The Angle Measure Between The Images of Two Curves in Case $q_{0}=\infty U \ldots \ldots \ldots \ldots \ldots \ldots \ldots$ 


\section{Chapter 1}

\section{Introduction}

\subsection{Overview and History}

Numerous problems of mathematical physics, e.g. mechanics, fluid mechanics, quantum mechanics, electricity magnetism and general relativity possess unbounded solutions. Therefore, a main goal of our research is to study objects that may have the untraditional form ; $\infty\left[\begin{array}{l}1 \\ 2 \\ 3 \\ 4\end{array}\right], \infty U$, where $U$ is a nonzero element of a function space. An unbounded object in a function space could also be a function $h$ on a finite or infinite interval. For example:

$$
h(s)=\exp \left(s-\frac{a+b}{2}\right)^{-2} \text { if } a \leq s \leq \frac{a+b}{2} \text {, and } h(s)=\infty \text { if } \frac{a+b}{2}<s \leq b .
$$

Bringing unbounded objects together with finite or bounded objects under one framework could reward the mathematical sciences. A rigorous treatment of such objects requires the extension of a Banach or Hilbert space into a closed metric space. Various metrics play a decisive role. We will compare them and show which ones are equivalent and which one are unexpectedly not equivalent. We will demonstrate the usefulness of our study by applications for example the approximation of unbounded functions. A formidable tool that is used throughout to achieve this goal is the construction of a plethora of nonlinear projections that take unbounded sets to bounded sets via certain bijections. This is in a nutshell the crux of this dissertation. 
The concept of a stereographic projection, where points of the "heavenly sphere" are matched in a one-to-one manner to the points of a plane passing through the center of the sphere, was known to Ptolemy about $200 \mathrm{CE}$ (T. J. Heath [29], page 292). Ptolemy (Claudius Ptolemaeus, born c. $100 \mathrm{CE}$ - died c. $170 \mathrm{CE}$ ) was motivated by astronomy. This is the earliest reference we could find where the unbounded set of points lying in a plane are mapped in a one-to-one manner onto the bounded set of points of a sphere (except the single fixed projection point that is either the north or south pole). In text books of complex function analysis, this sphere is called the Riemann sphere and the mapping is known as the stereographic projection. See e.g. L. V. Ahlfors [2] (page 19) and E. Hille [31] (pages 38-44) for derivations. In 1881, Poincare proposed a different mapping where the fixed projection point coincides with the center of the sphere. These two types of projection mappings are also referred to as "compactifications," as they map all points of the non-compact plane onto a bounded set of a sphere. Even though some of these projections have been known for approximately two thousand years, new applications have been found in the previous century and in this new millennium. The stereographic projection and and half sphere ( today knows as Poincaré's compactification) have been used to great advantage in $\mathbb{R}^{n}$ in theory and applications. For example, the Riemann sphere is utilized in $\mathbb{R}^{2}$ as a model for the extended complex plane. An induced metric is defined that provides a unified framework for the definition of convergence of bounded as well as unbounded sequences in $\mathbb{R}^{2}$. The stereographic projection has been applied to the study of systems of differential equations (I. Bendixon [8]). Several authors of ordinary differential equations textbooks, e.g. $[4,33,38,45,51]$, favor the utilization of the Poincaré compactification. Several researchers (C. Chicone and J. Sotomayor [11]; A. Cima and J. Lelibre [12]) utilized Poincaré's compactification to study solutions of systems of differential equations in the vicinity of infinity. The stereographic projection has also found relevance to the theory of quantum computation and quantum information, to conformal mappings, and to string theory in theoretical physics (J-w Lee et al. [37]), G. Najarbashi ,B. Seifi, and S. Mirzaei [41] and G. Najarbashi and B. Seifi, [42]. The human visual system is based on the sensation of light in the $3 \mathrm{D}$ physical world projecting onto the inner surface of our eyes. 2D artwork depicts 3D shapes via various forms of projection. Artists and computer graphics researchers study the projection methods employed in the past $[1,32]$. Artists and researchers employ nonlinear projection methods to create $2 \mathrm{D}$ images that match an artist's intent, 
such as to depict a scene as viewed from multiple points-of-view simultaneously or to better match human perceptual expectations $[1,10,58,61]$. The stereographic projection has been used for artistic purposes to create photographic effects [46]. Optical lenses produce non-linear projections [59]. These projections are investigated for the creation of affordable head-mounted displays for virtual and augmented reality [53,60]. The behavior of angles under projection (conformality) is of key importance in these applications. It was known to Ptolemy that the stereographic projection is conformal. A proof of the converse theorem, that if a projection of a two dimensional plane on a surface is conformal then the surface must necessarily be a sphere, was undertaken by E. Kasner and J. De Cicco [34].

New nonlinear projections-compactifications and new applications were found as well. See H. Gingold [24], where a paraboloid projection compactification was introduced and applied to approximation theory. See U. Elias and H. Gingold [15] for the application of the parabolic compactification to the "blow up" of solutions of systems of differential equations. Applications of a "parabolic projection" to nonlinear systems of finite differences were taken up by H. Gingold in [19-21]. The parabolic and other well known projectionscompactifications can be shown to be part of a larger framework of "radial compactifications" in $\mathbb{R}^{n}$ proposed in [15] . The blow up of solutions of systems of differential equations is an imporatnt criteria in Combustion theory. We defer to the references in J. Hell [30] and in A. Takayasu et al. [54]. Numerical validation of blow-up solutions of ordinary differential equations were obtained in A. Takayasu et al. [54]. New insights into the celebrated Lorenz system were found by H. Gingold and D. Solomon [22,23]. For comparisons among the stereographic projection, such as Poincare compactification, and the parabolic compactification, see Hell J. [30]. See also A. Takayasu et al. [54]. Projections of $\mathbb{R}^{n}$ on bounded sets have become indispensible tools for the definition and analysis of critical points "at infinity" of dynamical systems.

It is noteworthy that the new metric $\frac{d(h, \hat{h})}{1+d(h, \hat{h})}$ derived from the metric $d(h, \hat{h})$ of a given metric space is related to the mapping $\frac{x}{1+\|x\|}$ where \|\| is a suitable norm in a Banach space or a Hilbert space. It is a well-known powerful and favored tool in functional analysis. See e.g. E. Kreyszig [35] p.17 and H. L. Royden [50] page p. 186 and p.203. It is shown in here that the boundization $\frac{x}{1+\|x\|}$ emanates from a projection of a Hilbert space into a bounded subset of a "cone". 
The several boundizations and "compactifications" in use today in mathematics proper and in the mathematical sciences raise several questions of great importance. Are the metrics induced by the cone projections, the "Half Sphere" (Poincaré boundization) and the Stereographic Projection, equivalent? Does convergence to a critical point of a dynamical system in one of these metrics imply automatically convergence in the other metrics ?. If these limited number of boundizations are not equivalent, how can one make available in the mathematical sciences larger families of boundizations for comparison and use? At the core of this framework lies an extension of a Banach and Hilbert space to certain metric spaces with new elements of unconventional form and use and endowed with a new metric. The metric spaces being constructed by nonlinear transformations. In this respect our work is a contribution to nonlinear analysis. The source of these nonlinear transformations are no other than surfaces defined in a setting of an extended Hilbert space that originate geometrically from the conic sections defined in a Cartesian plane by certain distances from foci or by distances from a focus and a straight line.

A comment on the term "compactification" is necessary. Whilst it is proper to call a mapping of $\mathbb{R}^{n}$ into a bounded set a "compactification", it could be misleading and confusing to continue to call a mapping of an infinite dimensional space into a bounded set a "compactification". It is well known that the unit ball in a Hilbert space is not a compact set, and the image of the unit ball may not be compact either. This echoes J. Hell [30] who expressed concern about the usage of the word "compactification". Therefore, we add a definition of a boundization. In 2007, Y. I. Gingold and H. Gingold [26] proposed and studied a family of nonlinear projections of the points of $\mathbb{R}^{2}$ onto subsets of a sphere in $\mathbb{R}^{3}$ that were "bowl" shaped. This family of projections depended on one real valued parameter. It turns out that the Poincaré projection and the radically different stereographic projection are obtained from two different particular values of this one parameter family by varying this parameter continuously in an interval. The relevance of these projections to perspective were discussed in [27]. In 2009 J. Hell [30] published her Ph.D. dissertation entitled "Conley Index at Infinity". Her dissertation suggested studying the stereographic projection and the Poincaré projection in the setting of a real Hilbert space.

It is also noteworthy that the extension of Euclidean and non-Euclidean geometry to complex Hilbert spaces attracted a substantial amount of attention. See Hahn [28] for Trigonometry on the unit ball of a complex Hilbert 
Space. This and other works mentioned in here should come as no surprise, given the special role of the sphere in the mathematical sciences.

The importance of angles in Euclidean geometry, in Hilbert spaces, and in the mathematical sciences, can hardly be over estimated. While the definition of an angle and its measure in Euclidean geometry and in a real Hilbert space is well established and accepted, the same does not hold true in a real Banach space and in a complex Hilbert space. The latter were subject to numerous investigations. A partial list includes J. E. Valentine and S. G. Wayment, [57], A. Galantai and Cs. J. Hegedus [17], and more recently V. W. Thurey [55], V. Balestro, H. Martini, and R. Teixeira, [6,7] and their references. Throughout this dissertation we adopt a definition of the measure of an angle $\mathcal{X}$ in a complex Hilbert space as follows:

Definition 1.1.1 Let $<,>$ be an inner product in a given complex Hilbert space $\mathcal{H}$. Let $\mathcal{V}, \hat{\mathcal{V}} \in \mathcal{H}$ be two unit vectors. Then, the measure of the angle $\mathcal{X}$ between any two arrows representing them is given by

$$
\mathcal{X}=\cos ^{-1}\left[\frac{\langle\mathcal{V}, \hat{\mathcal{V}}\rangle+\langle\hat{\mathcal{V}}, \mathcal{V}\rangle}{2}\right], \text { where } \cos (\mathcal{X})=\frac{\langle\mathcal{V}, \hat{\mathcal{V}}\rangle+\langle\hat{\mathcal{V}}, \mathcal{V}\rangle}{2}, 0 \leq \mathcal{X} \leq \pi
$$

Notice that $\cos (\mathcal{X})$ is a monotone decreasing function on the interval $0 \leq$ $\mathcal{X} \leq \pi$ and hence invertible. It is readily observed that $\cos (\mathcal{X})=\operatorname{Real}\langle\mathcal{V}, \hat{\mathcal{V}}\rangle$ and is real even in a complex Hilbert space. Moreover, in a real Hilbert space $\cos (\mathcal{X})=\langle\mathcal{V}, \hat{\mathcal{V}}\rangle$ is compatible with the definition of an angle in a real Hilbert space. Furthermore, (1.1.2) is consistent with the definition of an angle in Euclidean geometry. This definition of an angle in a complex Hilbert space and its ramifications are studied in chapter 7 and are also discussed at length in a publication by H. Gingold, Y. Gigold and S. Hamad [25] that is part of this dissertation.

Here is a chapter by chapter summary of the highlights of the thesis: In Chapter 2 the Ultra Extended Banach and the Ultra Extended Hilbert spaces and their properties are developed. We also investigate operations in the Ultra Extended Banach and Hilbert spaces. In chapter 3 Boundization and closure are defined and developed. We give five examples, motivated by conic sections in the plane. A Sphere, a paraboloid, a cone, a hyperboloid and an ellipsoid. In chapter 4 we discuss each example these families of boundization and we derive particular metrics for each one. Also we present approximating schemes in Banach spaces via boundizations. In chapter 5 we study the 
equivalence and non equivalence between two extended metrics. In chapter 6 we study the equivalence and non equivalence between extended metrics and a projection metrics. In chapter 7 we consider a family of nonlinear projections that map a complex Hilbert space onto a bounded bowl" shaped subset of a sphere. Our main result states that a projection is conformal if and only if it is the stereographic projection and if and only if the projection renders all certain pairs of triangles induced by the projection to be similar. It follows that various so called boundizations that are given in the literature are special members of this family of nonlinear projections. These include the stereographic projection and the Poincaré compactication. Background and motivation are discussed and several examples illustrating the results are provided.

Throughout this work we adopt the convention that $\mathbb{F}$ stands for the field $\mathbb{R}$ or $\mathbb{C}$. 


\section{Chapter 2}

\section{The Ultra Extended Banach and Hilbert Spaces}

\subsection{The Ultra Extended Banach Space}

In this chapter we extend a given Banach space $\mathbb{B}$ over $\mathbb{F}$. Denote by $I D$ the continuum of ideal points:

$$
I D=\{\infty U: U \in \mathbb{B} \text { with }\|U\|=1\}
$$

We will discuss in the sequel why every element $\infty U \in I D$ actually represents an equivalence classes. A few examples follow.

Example 2.1.1 If $\mathbb{B}=\mathbb{R}$ over $\mathbb{R}$ then

$$
I D=\{\infty(-1), \infty(1)\}
$$

Example 2.1.2 $\mathbb{B}=\mathbb{R}^{2}$ over $\mathbb{C}$ then

$$
I D=\left\{\infty e^{i \phi}, 0 \leq \phi<2 \pi\right\}
$$

Definition 2.1.1 the set $\mathbb{B} \cup I D$ is called the Ultra Extended Banach space and denoted by $\mathbb{U E B}$.

Let $q \in \mathbb{B}$ and denote by $\|q\|$ its norm. In the sequel we employ three related linear vector spaces $\mathbb{B}, \mathbb{B}_{N}, \mathbb{S}_{N}$. Let $u$ be a fixed non zero-element of $\mathbb{B}$. Denote by $\mathbb{B}_{N}$ a new set defined by $\mathbb{B}_{N}:=\{c u\} \times \mathbb{B}$ where $c \in \mathbb{F}$. It is easily recognized that the subset of elements of the form $(\overrightarrow{0}, q) \in \mathbb{B}_{N}, q \in \mathbb{B}$, 
to be denoted by $\mathbb{S}_{N}$, forms a subspace of $\mathbb{B}_{N}$, then $\mathbb{S}_{N}=\{\overrightarrow{0}\} \times \mathbb{B}$. Moreover, $\mathbb{S}_{N}$ is isomorphic to $\mathbb{B}$. Let $Z \in \mathbb{B}_{N}$, we view $Z$ as a point in $\mathbb{B}_{N}$ as well as a vector. Each elements in $\mathbb{B}_{N}$ has the form $Z=(c u, z) \in \mathbb{B}_{N}$ for all $z \in \mathbb{B}$. We define a new additional operation $\boxplus$ in $\mathbb{B}_{N}$ as follows

$$
Z \boxplus \hat{Z}=(c u+\hat{c} u, z+\hat{z})
$$

We also add a new multiplication operation $\odot$ in $\mathbb{B}_{N}$ defined as follows

$$
a \odot Z=(a c u, a z) \text { where } a \in \mathbb{F} \text {. }
$$

It is easy to check that with the addition and scalar multiplication defined above in $\mathbb{B}_{N}$, all axioms of a linear space hold. Henceforth we replace in $\mathbb{B}_{N}$ the new operations symbols $\boxplus, \odot$ with the old symbols of,.+ in $\mathbb{B}$. We denote by $\overrightarrow{0}$ the identity element in $\mathbb{B}$ with respect to the addition operation + . This, with the understanding that it is not to be confused with the + the addition operation in $\mathbb{F}$ and with its 0 identity element in $\mathbb{F}$. We define a new norm in $\mathbb{B}_{N}$ as follows. Let $Z \in \mathbb{B}_{N}$ such that $Z=(c u, z)$

$$
\|Z\|_{\mathbb{B}_{N}}=\|c u\|+\|z\|
$$

It's easy to check that this defines a norm in $\mathbb{B}$. For any $Z=(c u, z), \hat{Z}=$ $(\hat{c} u, \hat{z}), c, \hat{c} \in \mathbb{F}$ we have

1. If $\|Z\|_{\mathbb{B}_{N}}=\|c u\|+\|z\|=0$ then $c=0$ and $z=0$ therefore $Z=\hat{0}$, the neutral element in $\mathbb{B}_{N}$.

2. Since $\|c u\| \geq 0$ for all $u \in \mathbb{B}$ and $\|z\| \geq 0$ for all $z \in \mathbb{B}$ then $\|Z\|_{\mathbb{B}_{N}} \geq 0$ for all $Z \in \mathbb{B}_{N}$

3. $\|a Z\|_{\mathbb{B}_{N}}=\|a c u\|+\|a z\|=|a|(\|c u\|+\|z\|)=|a|\|Z\|_{\mathbb{B}_{N}}$

4. $\|Z+\hat{Z}\|_{\mathbb{B}_{N}}=\|c u+\hat{c} u\|+\|z+\hat{z}\| \leq\|c\|+\|\hat{c}\|+\|z\|+\|\hat{z}\|=\|Z\|_{\mathbb{B}_{N}}+\|\hat{Z}\|_{\mathbb{B}_{N}}$

Proposition 2.1.1 Let \|\| be the norm of a Banach space $\mathbb{B}$ over the field $\mathbb{F}$. Let $u \in \mathbb{B}$ be any non zero fixed element. Then, the Cartesian product $\{c u\} \times \mathbb{B}$, where $c \in \mathbb{F}$, is a Banach space over $\mathbb{F}$ with the following induced norm.

$$
\|Z\|_{\mathbb{B}_{N}}=\|c u\|+\|z\|
$$


Proof We are left to prove completeness in $\mathbb{B}_{N}$. Let $Z_{n}=\left(c_{n} u, z_{n}\right)$ be a Cauchy sequence in this norm. Let $\epsilon>0$ and let $N \in \mathbb{N}$ be such that for all $m, n \geq N$ we have

$$
\left\|Z_{n}-Z_{m}\right\|_{\mathbb{B}_{N}}<\epsilon
$$

Then by the definition of our norm we must also have

$$
\left\|c_{n} u-c_{m} u\right\|+\left\|z_{n}-z_{m}\right\|<\epsilon,
$$

and hence $c_{n} u$ and $z_{n}$ are Cauchy sequences in $\mathbb{B}$ so they are convergent therefore $Z_{n}$ is convergent in $\mathbb{B}_{N}$. The result follow.

\subsection{The Ultra Extended Hilbert Space}

Let $q \in \mathbb{H}$ and denote by $\|q\|=\sqrt{\langle q, q\rangle}$ the norm of $q$. In the sequel we employ three related linear vector spaces $\mathbb{H}, \mathbb{H}_{N}, \mathbb{S}_{N}$. Let $u \neq \overrightarrow{0}$ be a unit element of $\mathbb{H}$, namely $\langle u, u\rangle=1$. Consider the one dimensional subspace $\mathbb{K}$

$$
\mathbb{K}=\{c u: u \in \mathbb{H},\|u\|=1, c \in \mathbb{F}\} .
$$

Denote by $\mathbb{H}_{N}$ a new set defined by $\mathbb{H}_{N}:=\mathbb{K} \times \mathbb{H}$. Let $Z \in \mathbb{H}_{N}$ we view $Z$ as points in $\mathbb{H}_{N}$ as well as vectors. Every elements in $\mathbb{B}_{N}$ has the form $Z=(c u, z) \in \mathbb{H}_{N}$ for all $c \in \mathbb{F}$ and $z \in \mathbb{H}$. Define in $\mathbb{H}_{N}$ the operation

$$
\langle Z, \hat{Z}\rangle_{H_{N}}=\langle z, \hat{z}\rangle+\langle c u, \hat{c} u\rangle=\langle z, \hat{z}\rangle+c \overline{\hat{c}} .
$$

It can be verified that with the above definition all axioms of an inner product space hold in $\mathbb{H}_{N}$. Let $Z, \hat{Z}$ and $\tilde{Z}$ belong to $\mathbb{H}_{N}$ such that $Z=(c u, z)$, $\hat{Z}=(\hat{c} u, \hat{z})$ and $\tilde{Z}=(\tilde{c} u, \tilde{z})$. Then,

1.

$$
\begin{aligned}
\langle Z \boxplus \hat{Z}, \tilde{Z}\rangle_{H_{N}} & =\langle z+\hat{z}, \tilde{z}\rangle+\langle c u+\hat{c} u, \tilde{c} u\rangle \\
& =\langle z, \tilde{z}\rangle+\langle\hat{z}, \tilde{z}\rangle+\langle c u, \hat{c} u\rangle+\langle\hat{c} u, \tilde{c} u\rangle \\
& =\langle Z, \tilde{Z}\rangle_{H_{N}} \boxplus\langle\hat{Z}, \tilde{Z}\rangle_{H N}
\end{aligned}
$$

2. let $a \in \mathbb{F}$ than we have

$$
\begin{aligned}
\langle a \odot Z, \hat{Z}\rangle_{H_{N}} & =\langle a z, \hat{z}\rangle+\langle a c u, \hat{c} u\rangle \\
& =a(\langle z, \hat{z}\rangle+\langle c u, \hat{c} u\rangle)=a \odot\langle Z, \hat{Z}\rangle_{H_{N}}
\end{aligned}
$$


3.

$$
\begin{aligned}
\langle Z, \hat{Z}\rangle_{H_{N}} & =\langle z, \hat{z}\rangle+\langle c u, \hat{c} u\rangle=\overline{\langle\hat{z}, z\rangle}+\overline{\langle\hat{c} u, c u\rangle} \\
& =\overline{\langle\hat{z}, z\rangle+\langle\hat{c} u, c u\rangle}=\overline{\langle\hat{Z}, Z\rangle_{H_{N}}}
\end{aligned}
$$

4. $\langle Z, \hat{Z}\rangle_{H_{N}}=\langle z, \hat{z}\rangle+\langle c u, \hat{c} u\rangle$ since $\langle z, \hat{z}\rangle \geq 0$ and $\langle c u, \hat{c} u\rangle \geq 0$ so $\langle Z, \hat{Z}\rangle_{H_{N}} \geq 0$, also $\langle z, z\rangle=0$ if and only if $z=\overrightarrow{0}$ and $\langle c u, c u\rangle=0$ if and only if $c=0$ thus $\langle Z, Z\rangle_{H_{N}}=0$ if and only if $Z=\overrightarrow{0}$.

Denote by $\|Z\|_{H_{N}}=\sqrt{\langle Z, Z\rangle_{H_{N}}}$. We will verify that indeed $\|Z\|_{H_{N}}$ is a norm on $\mathbb{H}_{N}$ as follows.

1. $\|Z\|_{H_{N}} \geq 0$

2. $\|Z\|_{H_{N}}=0$ if and only if $Z=0$

3. $\|a Z\|_{H_{N}}=\sqrt{\langle a Z, a Z\rangle_{H_{N}}}=\sqrt{a \bar{a}} \sqrt{\langle Z, Z\rangle_{H_{N}}}=|a| \sqrt{\langle Z, Z\rangle_{H_{N}}}$

4. It is well known that the Cauchy Schwartz inequality See e.g. E. Kreyszig [35] page 137

$$
\left|\langle Z, \hat{Z}\rangle_{H_{N}}\right| \leq\|Z\|_{H_{N}}\|\hat{Z}\|_{H_{N}}
$$

holds in a inner product space and consequently the triangle inequality is implied

$$
\|Z+\hat{Z}\|_{H_{N}} \leq\|Z\|_{H_{N}}+\|\hat{Z}\|_{H_{N}} .
$$

Remark Assume that $Z=(c u, \overrightarrow{0})$ and $\hat{Z}=(\overrightarrow{0}, \hat{z})$ are two vectors in $\mathbb{H}_{N}$ .Then $\langle Z, \hat{Z}\rangle_{H_{N}}=0$ for all $c u \in \mathbb{K}, z$ and $\hat{z} \in \mathbb{H}$. It is evident that $Z$ and $\hat{Z}$ are orthogonal. Moreover, $\mathbb{S}_{N}=\{\overrightarrow{0}\} \times \mathbb{H}$ is isomorphic to $\mathbb{H}$ - We may refer to the one dimensional set Axis: $Z Z Z=(c u, \overrightarrow{0}), c \in \mathbb{F}$ as the axis subspace of $\mathbb{H}_{N}$, and to $\mathbb{S}_{N}$ as the "plane" $\mathbb{S}_{N}$ in $\mathbb{H}_{N}$. Evidently $\mathbb{H}_{N}=$ Axis $\oplus \mathbb{S}_{N}$, Axis $\perp \mathbb{H}_{N}$.

Definition 2.2.1 the set $\mathbb{H} \cup I D$ is called the Ultra Extended Hilbert space and denoted by $\mathbb{U E H}$.

It is easy to verify that the set $\mathbb{U E B}$ may be written in the form

$$
\mathbb{U} \mathbb{E} B=\{\{\rho U, U \in \mathbb{B},\|U\|=1,0<\rho \leqslant \infty\} \cup\{\overrightarrow{0}\}\}
$$

This parametrization is the analog of a "polar" parametrization of points in a Cartesian plane. Each element $w \in \mathbb{B} \backslash\{\overrightarrow{0}\}$ has a unique representation $\rho U, w=\|w\|\|w\|^{-1} w$ such that $U=\|w\|^{-1} w$ and $\rho=\|w\|$. 


\subsection{Operations in the Ultra Extended Banach and Hilbert Spaces}

One should not expect an $\mathbb{U E \mathbb { B }}$ or an $\mathbb{U} \mathbb{E} \mathbb{H}$ to preserve all properties of a linear vector space or of a Banach space or a Hilbert space. However, the operations of addition and multiplication by a scalar still hold for certain subsets of elements in the $\mathbb{U E \mathbb { B }}$ on which we elaborate in the sequel. Therefore, one should not expect the addition of two elements in $\mathbb{U E B}$ and the scalar multiplication of an element in $\mathbb{U} \mathbb{E} \mathbb{B}$ to be well defined for all elements in ID. We propose a lemma that allows us to perform the operation addition on various sequences of elements. The lemma below shows that the partial sums of series $\sum_{j=1}^{\infty} v_{j}, v_{j} \in \mathbb{B}$ are well defined if all $v_{j}$ lies in half a plane.

Lemma 2.3.1 Let $v_{j}$ be such that $v_{j} \neq 0$ and $v_{j} \in \mathbb{H}$, and assume that $0 \leq \phi_{j} \leq \frac{\pi}{2}-\epsilon$ where $\phi_{j}$ is the angel between $v_{j}$ and a fixed normal vector $N \neq 0, j=1,2, \ldots, m$ then

1. $\sum_{j=1}^{m} v_{j} \neq 0$

2. The angle $\phi$ between $\sum_{j=1}^{m} v_{j}$ and $N$ is such that $0 \leq \phi \leq \frac{\pi}{2}-\epsilon$.

Proof For the first part of this lemma it is sufficient to discuss $m=2$. the rest follows by induction. By the way of contradiction, assume that $v_{1}+v_{2}=0$ then

$$
0=\left\langle v_{1}+v_{2}, \frac{N}{\|N\|}\right\rangle=\left\langle v_{1}, \frac{N}{\|N\|}\right\rangle+\left\langle v_{2}, \frac{N}{\|N\|}\right\rangle
$$

and

$$
0=\left\langle\frac{N}{\|N\|}, v_{1}+v_{2}\right\rangle=\left\langle\frac{N}{\|N\|}, v_{1}\right\rangle+\left\langle\frac{N}{\|N\|}, v_{2}\right\rangle
$$

Thus

$$
\begin{aligned}
0 & =\frac{\left(\left\langle\frac{v_{1}}{\left\|v_{1}\right\|}, \frac{N}{\|N\|}\right\rangle+\left\langle\frac{N}{\|N\|}, \frac{v_{1}}{\left\|v_{1}\right\|}\right\rangle\right)\left\|v_{1}\right\|}{2}+\frac{\left(\left\langle\frac{v_{2}}{\left\|v_{2}\right\|}, \frac{N}{\|N\|}\right\rangle+\left\langle\frac{N}{\|N\|}, \frac{v_{2}}{\left\|v_{2}\right\|}\right\rangle\right)\left\|v_{2}\right\|}{2} \\
& =\left\|v_{1}\right\| \cos \phi_{1}+\left\|v_{2}\right\| \cos \phi_{2}
\end{aligned}
$$

since $\left\|v_{1}\right\| \neq 0,\left\|v_{2}\right\| \neq 0,0 \leq \phi_{1} \leq \frac{\pi}{2}-\epsilon$ and $0 \leq \phi_{2} \leq \frac{\pi}{2}-\epsilon$ then $\cos \phi_{1} \neq 0$ and $\cos \phi_{2} \neq 0$, thus $\left\|v_{1}\right\| \cos \phi_{1}+\left\|v_{2}\right\| \cos \phi_{2}>0$ a contradiction. therefore 
$v_{1}+v_{2} \neq 0$. Now we assume that the statement holds for $m=k$ thus lead $\sum_{j=1}^{k} v_{j} \neq 0$, since $\sum_{j=1}^{k} v_{j} \neq 0$ is an element in $\mathbb{H}$, then for $v_{k+1} \neq 0$ we have $\left(\sum_{j=1}^{k} v_{j}\right)+v_{k+1} \neq 0$. Thus the statement holds for $m=k+1$, therefore the proof of induction step is complete. Now, we prove the second part of this lemma. By assumption we have

$$
\delta_{\epsilon} \leq \cos \phi_{j}=\frac{\left\langle\frac{v_{j}}{\left\|v_{j}\right\|}, \frac{N}{\|N\|}\right\rangle+\left\langle\frac{N}{\|N\|}, \frac{v_{j}}{\left\|v_{j}\right\|}\right\rangle}{2} \quad j=1,2, \ldots, m
$$

therefore

$$
\begin{aligned}
\cos \phi & =\frac{\left\langle v_{1}, N\right\rangle+\left\langle N, v_{1}\right\rangle+\left\langle v_{2}, N\right\rangle+\left\langle N, v_{2}\right\rangle+\cdots+\left\langle v_{m}, N\right\rangle+\left\langle N, v_{m}\right\rangle}{2\left\|v_{1}+v_{2}+\cdots+v_{m}\right\|\|N\|} \\
& =\frac{\left\|v_{1}\right\|\|N\|\left(\left\langle\frac{v_{1}}{\left\|v_{1}\right\|}, \frac{N}{\|N\|}\right\rangle+\left\langle\frac{N}{\|N\|}, \frac{v_{1}}{\left\|v_{1}\right\|}\right\rangle\right)}{2\left\|v_{1}+v_{2}+\cdots+\right\|\|N\|}+\cdots+ \\
& +\frac{\left\|v_{m}\right\|\|N\|\left(\left\langle\frac{v_{m}}{\left\|v_{m}\right\|}, \frac{N}{\|N\|}\right\rangle+\left\langle\frac{N}{\|N\|}, \frac{v_{m}}{\left\|v_{m}\right\|}\right\rangle\right)}{2\left\|v_{1}+v_{2}+\cdots+v_{m}\right\|\|N\|} \\
& =\frac{\left\|v_{1}\right\|\left(\left\langle\frac{v_{1}}{\left\|v_{1}\right\|}, \frac{N}{\|N\|}\right\rangle+\left\langle\frac{N}{\|N\|}, \frac{v_{1}}{\left\|v_{1}\right\|}\right\rangle\right)}{2\left\|v_{1}+v_{2}+\cdots+\right\|}+\cdots+ \\
& +\frac{\left\|v_{m}\right\|\left(\left\langle\frac{v_{m}}{\left\|v_{m}\right\|}, \frac{N}{\|N\|}\right\rangle+\left\langle\frac{N}{\|N\|}, \frac{v_{m}}{\left\|v_{m}\right\|}\right\rangle\right)}{2\left\|v_{1}+v_{2}+\cdots+v_{m}\right\|} \\
& =\frac{\left\|v_{1}\right\|\left(\cos \phi_{1}\right)+\left\|v_{2}\right\|\left(\cos \phi_{2}\right)+\cdots+\left\|v_{m}\right\|\left(\cos \phi_{m}\right)}{\left\|v_{1}+v_{2}+\cdots+v_{m}\right\|} \\
& \geq \frac{\left\|v_{1}\right\| \delta_{\epsilon}+\left\|v_{2}\right\| \delta_{\epsilon}+\cdots+\left\|v_{m}\right\| \delta_{\epsilon}}{\left\|v_{1}+v_{2}+\cdots+v_{m}\right\|} \geq \delta_{\epsilon}>0 .
\end{aligned}
$$

Definition 2.3.1 We define an addition and a scalar multiplication operations in an $\mathbb{U E H}$ and in an $\mathbb{U E \mathbb { B }}$ that use the symbol $\infty U$ as follows:

1.

$$
\infty U+q=q+\infty U=\infty U
$$

2.

$$
c \infty U \in \begin{cases}\infty(-U) & \text { if } c<0 \\ \infty U & \text { if } c>0\end{cases}
$$


where $\|U\|=1$.

It is noteworthy that algebraic operations with elements infinity are of interest to convexity theory. See e.g. R. T. Rockafellar [48] section 4. A non-empty bounded subset of a linear vector space cannot be a linear vector subspace of a linear vector space over the field $\mathbb{R}$ or $\mathbb{C}$ because a non empty vector space must be unbounded. A boundization requires a bijection from $\mathbb{U E B}$ onto a bounded subset of $\mathbb{B}_{N}$. The second reason being that $\infty U-\infty U$ and $\overrightarrow{0} \infty U$ are not well defined objects. Therefore, an $\mathbb{U E \mathbb { B }}$ cannot be isomorphic to a linear vector space and cannot be a linear vector space on its own right. Neither can its image in $\mathbb{B}_{N}$, under a bijection, to be a linear vector space. The important ramification being that the theories developed for Banach and Hilbert spaces do not apply to the $\mathbb{U E \mathbb { B }}$ or to its image in $\mathbb{B}_{N}$, and requires an additional analysis. This is a main undertaking of this dissertation.

\subsection{Convergence in the Ultra Extended Ba- nach and Hilbert Spaces}

We have been familiar with the concept of convergence, which deals with a sequences approach to a certain value. Since we deals with an element in the $\mathbb{U E B}$, then we need to come to some agreement about the symbol $\infty U$. Two definitions of convergence to infinity in Banach spaces are formulated below.

Definition 2.4.1 Let \|\| be the norm of a Banach space $\mathbb{B}$ over the field of real numbers $\mathbb{R}$ or $\mathbb{C}$. We say that the sequence $q_{n}$ converges to infinity in the degenerate sense if

$$
\lim _{n \rightarrow \infty}\left\|q_{n}\right\|=\infty
$$

We say that $q_{n}$ converges to infinity in the extended sense if (2.4.1) holds

and $\frac{q_{n}}{\left\|q_{n}\right\|}$ is a Cauchy sequence in $\mathbb{B}$. Namely, there exists $U \in \mathbb{B}$ such that

$$
\lim _{n \rightarrow \infty} \frac{q_{n}}{\left\|q_{n}\right\|}=U,\|U\|=1
$$

Remark The definition of a sequence of real numbers (covering) diverging to $\infty$ or to $-\infty$ in an extended $\mathbb{R}^{1}$ is well established in elementary calculus and is widely accepted. We cannot say the same about convergence to infinity 
in $\mathbb{R}^{n}$, in $\mathbb{C}^{n}$ or in an abstract Banach or Hilbert space. What does the convergence of a sequence $\left\{V_{n} \in \mathbb{R}^{n}\right\}, n=1,2, \cdots$ to infinity mean? Are there different objects infinity? According to W. F. Trench [56] chapter 5 page 306, it means that $\lim _{n \rightarrow \infty}\left\|V_{n}\right\|=\infty$ where \|\| is the Euclidean norm. This is akin to a definition compatible with the stereographic projection. The theory of ODE's has provided a stimulus to the reevaluation of these issues. Evidently, a sequence that converges to $\infty U$ in the extended sense also converges to infinity in the degenerate sense but not conversely. Closing this gap is an imporatnt goal of this thesis. See e.g. Trench part II [56], for the definition of convergence to infinity in $\mathbb{R}^{n}, n>1$ in the degenerate sense and consult chapter 3 section 13 page 79 in Ross [49]. Also compare with text books like [35,50].

For the sake of uniform verbiage we adopt the convention that a sequence converges to infinity or to $\infty U$ rather than use the term divergence to infinity as used in calculus.

\subsection{Sequences and Series in $\mathbb{U E} \mathbb{B}$}

We demonstrate the machinery that is developed in this dissertation by several examples. The next example shows the fundamental difference between convergence in the degenerate sense and convergence in the extended sense.

Example 2.5.1 The scalar sequence $q_{n}=(-1)^{n} n$ converges in the degenerate sense to infinity but does not converge to infinity in the extended sense, because

$$
\lim _{n \rightarrow \infty}\left\|q_{n}\right\|=\lim _{n \rightarrow \infty} n=\infty
$$

Example 2.5.2 The sequence

$$
q_{n}=e^{n}\left(\cos \left(\frac{\pi}{4} n\right), \sin \left(\frac{\pi}{4} n\right)\right)
$$

converges in $l^{2}$ to infinity in the degenerate sense but does not converge in the extended sense, because

$$
\lim _{n \longrightarrow \infty}\left\|q_{n}\right\|=\lim _{n \longrightarrow \infty} e^{n} \sqrt{\cos ^{2}\left(\frac{\pi}{4} n\right)+\sin \left(\frac{\pi}{4} n\right)}=\lim _{n \longrightarrow \infty} e^{n}=\infty
$$

The above demonstrates the huge difference between convergence in the degenerate sense and convergence in the extended sense. 
Example 2.5.3 Let $U_{1}, U_{2}$ be distinct unit vectors in a Banach space $\mathbb{B}$ over $\mathbb{R}$ or $\mathbb{C}$. Define as follows an infinite sequence $q_{n}, n=1,2, \ldots$ in $\mathbb{U} \mathbb{E} \mathbb{B}$,

$$
q_{2 k-1}=\infty U_{1}, q_{2 k}=\frac{1}{k+1} U_{2}, k=1,2,3, \ldots
$$

Then, $\overrightarrow{0}$ is the only accumulation point of the set

$$
\left\{\infty U_{1}, q_{2 k}=\frac{1}{k+1} U_{2}, k=1,2,3, \ldots\right\}
$$

in $\mathbb{U E} \mathbb{B}$ and $\left\{\overrightarrow{0}, \infty U_{1}\right\}$ is the set of limit points in $\mathbb{U E \mathbb { B }}$.

Example 2.5.4 Let $U$ be a unit vector in a Banach space $\mathbb{B}$ over $\mathbb{R}$ or $\mathbb{C}$. Define as follows an infinite sequence $q_{n}, n=1,2, \ldots$ in $\mathbb{U E \mathbb { B }}$,

$$
q_{2 k-1}=\infty U, q_{2 k}=\infty(-U), k=1,2,3, \ldots
$$

Then, the partial sums of the above sequence are not well defined.

Example 2.5.5 Define an infinite sequence $q_{n}, n=1,2, \ldots$ in $\mathbb{U} \mathbb{E} \mathbb{B}$ by specifying $q_{1}=\infty U, U \in \mathbb{B},\|U\|=1$ and $q_{n} \in \mathbb{B}, n=2,3 \ldots$ where (the first $k$ below starts with 2)

$$
b \in \mathbb{B}, \text { and } \lim _{n \rightarrow \infty}\left\|b-\sum_{k=2}^{n} q_{k}\right\|=0 .
$$

Then, $\sum_{k=1}^{\infty} q_{k}=q_{1}=\infty U$.

Next we have

Example 2.5.6 Let $U$ be a unit vector in a Banach space $\mathbb{B}$ over $\mathbb{R}$ or $\mathbb{C}$. Define as follows an infinite sequence $q_{n}, n=1,2, \ldots$ in $\mathbb{U} \mathbb{E}$,

$$
q_{2 k-1}=\infty U, q_{2 k}=\infty(-U), k=1,2,3, \ldots .
$$

Then, the series $\sum_{k=1}^{\infty} q_{k}$ are not well-defined. 


\section{Chapter 3}

\section{Boundization and Closure}

For our research, it is important to explore the function between bounded and unbounded spaces. The purpose of this chapter is that we first present a general surface and then we define the relation between any point on a surface to a fixed point in a subset of the Ultra Extended Hilbert space. We will use this relation to explain the family of boundization for this surface. Second we present five different examples of families of surfaces, namely a surface of sphere, paraboloid, cone, hyperboloid and ellipsoid.

\subsection{What are Normalized Boundizations?}

A normalized boundization of a Banach space is a mapping $z=t q$ from $\mathbb{U E \mathbb { B }}$ to a bounded subset of $\mathbb{B}$ with a scalar function $t=t(\|q\|)$ defined on the interval $[0, \infty)$ such that

1. The mapping $\psi(\|q\|):=t(\|q\|)\|q\|$ is a monotone non negative bijection from the semi infinite interval $0 \leq\|q\|<\infty$ to a certain interval $0 \leq \psi<L<\infty$ and such that

$$
\lim _{\|q\| \rightarrow \infty} \psi(\|q\|)=L .
$$

2. $t(\|q\|)$ is Lipschitz. Namely, there exists a constant $M_{1}>0$ such that for $0 \leq\|q\|,\|\hat{q}\|<\infty$ we have

$$
|t(\|q\|)-t(\|\hat{q}\|)| \leq M_{1}\|q-\hat{q}\| .
$$


Proposition 3.1.1 The mapping $\mathcal{G}$ from $\mathbb{S}_{N}$ into $\mathbb{B}_{N}$ is given by

$$
\mathcal{G}((\overrightarrow{0}, q)):=((1-t) \gamma, t q)
$$

is a bijection.

Proof If $\mathcal{G}((\overrightarrow{0}, q))=\mathcal{G}((\overrightarrow{0}, \hat{q}))$ where $q$ and $\hat{q} \in B$ then $((1-t) \gamma, t(\|q\|) q)$ $=((1-t) \gamma, t(\|\hat{q}\|) \hat{q})$ therefore $t(\|q\|) q=t(\|\hat{q}\|) \hat{q}$, thus $q=\hat{q}$. Moreover, for all $((1-t) \gamma, t q) \in \mathbb{B}_{N}$ there exist $(\overrightarrow{0}, q) \in \mathbb{S}_{N}$ such that $\left.\mathcal{G}(\overrightarrow{0}, q)\right):=((1-t) \gamma, t q)$

Example 3.1.1 Here is an example of a normalized boundization that will be derived in the sequel by geometric considerations. A "cone" boundization with

$$
z(q)=z=t(\|q\|) q, t(\|q\|)=\frac{\eta}{\|q\|+\tau}, \eta>0, \tau>0,
$$

Remark Assuming that we are given a normalized boundization of a Banach space it is possible to show that the distance between two elements $\infty U$ and object $\infty V, V \neq \hat{0}$ where $U=\|V\|^{-1} V$ is zero.

\subsection{Closure}

$\mathbb{U E \mathbb { B }}$ may be thought of as the closure of $\mathbb{B}$, where neighborhoods in $\mathbb{U E \mathbb { B }}$ are determined by either the projection metric or by the extended metric. The points in $I D$ are accumulation points of $\mathbb{U E} \mathbb{B}$ that are not in $\mathbb{B}$ and are the precise boundary of $\mathbb{B}$.

Remark We provide an interpretation to the ideal set $I D$ in $\mathcal{L}(\mathbb{H}, \mathbb{H})$, the linear space of linear operators, of a finite dimensional inner product space $\mathbb{H}$ over $\mathbb{C}$ that is endowed with the Frobenius inner product. It is sufficient to consider $\mathbb{H}$ to be the linear space of $n \times n$ matrices over $\mathbb{C}$. See e.g. S. Axler [5] section 4 . This is so because any linear space $\mathcal{L}(\mathbb{H}, \mathbb{H})$ is isomorphic to the linear space of $n x n$ matrices. Let $A \in \mathcal{L}(\mathbb{H}, \mathbb{H})$. Put $A=\left(a_{j k}\right), j, k=$ $1,2, \ldots, d$. Then, $\operatorname{Trace}\left(A^{*} A\right)=\sum_{k=1}^{d} \sum_{j=1}^{d}\left|a_{j k}\right|^{2}$. If in addition $A$ is a normal matrix, then

$$
A^{*} A=A^{*} A \Rightarrow \operatorname{Trace}\left(A^{*} A\right)=\sum_{k=1}^{d} \sum_{j=1}^{d}\left|a_{j k}\right|^{2}=\sum_{j=1}^{d}\left|\lambda_{j}\right|^{2},
$$


where $\lambda_{j}, j=1,2, \ldots, d$ are the eigenvalues of the matrix $A$. Hence, a unit element $U=\left[\sqrt{\operatorname{Trace}\left(A^{*} A\right)}\right]^{-1} A$ and $\infty\left[\sqrt{\operatorname{Trace}\left(A^{*} A\right)}\right]^{-1} A$ is the limit of sequences of matrices of the form $\rho\left[\sqrt{\operatorname{Trace}\left(A^{*} A\right)}\right]^{-1} A$ where $A$ is fixed with at least one non zero element and $\rho \rightarrow \infty$. In particular, if $A$ coincides with $E_{u v}=\left(e_{j k}\right), e_{j k}=0$ if $(j, k) \neq(u, v)$ and $e_{j k}=1$ if $(j, k)=(u, v)$ then

$$
\infty U=\infty E_{u v}=\left(\widehat{e}_{j k}\right), \widehat{e}_{j k}=0 \quad \text { if } \quad(j, k) \neq(u, v)
$$

and

$$
\widehat{e}_{j k}=\infty \quad \text { if } \quad(j, k)=(u, v) .
$$

If $A^{*} A=A^{*} A$ then $U=\left[\sqrt{\operatorname{Trace}\left(A^{*} A\right)}\right]^{-1} A$.But then $A=P^{*} \Lambda P$ with $P^{*} P=I$ so that

$$
\begin{aligned}
U & =\left[\sqrt{\operatorname{Trace}\left(P^{*} \Lambda^{*} P P^{*} \Lambda P\right)}\right]^{-1} P^{*} \Lambda P \\
& =\left[\sqrt{P^{*} \operatorname{Trace}\left(\Lambda^{*} \Lambda\right) P}\right]^{-1} P^{*} \Lambda P \\
& =\left[\sqrt{P^{*} \operatorname{PTrace}\left(\Lambda^{*} \Lambda\right)}\right]^{-1} P^{*} \Lambda P=\left[\sqrt{\operatorname{Trace}\left(\Lambda^{*} \Lambda\right)}\right]^{-1} P^{*} \Lambda P \\
& =P^{*}\left[\sqrt{\operatorname{Trace}\left(\Lambda^{*} \Lambda\right)}\right]^{-1} \Lambda P .
\end{aligned}
$$

Thus, $\infty U$ is the limit of $\rho P^{*}\left[\sqrt{\operatorname{Trace}\left(\Lambda^{*} \Lambda\right)}\right]^{-1} \Lambda P$ where $P$ is a unitary fixed matrix, $\Lambda$ is a diagonal matrix with at least one non zero eigenvalue and $\rho \rightarrow \infty$.

\subsection{Banach Spaces versus Hilbert Spaces}

As seen from the definition of a normalized boundization above, it is defined independently of geometrical considerations. However, historically these boundizations were inspired by geometrical construction of projections of a line on a circle or the projection of a plane on a sphere, and were known to Helenic mathematicians. We continue this tradition and proceed to discover new boundizations for Banach spaces by utilizing geometrical constructions defining families of surfaces in Hilbert spaces. Based on the latter, natural reinterpretation of these boundizations in Banach and Hilbert spaces will follow. 


\subsection{A General Surface Boundization and Ge- ometrical Considerations}

We first adopt some nomenclature and notation that gives us a general idea about the relation between a point on a surface and a point in a subset of the $\mathbb{U} \mathbb{E} \mathbb{H}$. Assume that $P=\left(\gamma_{g} u, \overrightarrow{0}\right)$ be a fixed point in $\mathbb{H}_{N}$ where $\gamma_{g} \in \mathbb{C}$ is fixed and $Q=(\overrightarrow{0}, q)$ be an arbitrary point in $\mathbb{U E H}$ and let $Z=(c u, z) \in \mathbb{H}_{N}$ lies on a general surface in $\mathbb{H}_{N}$. If we normalize the setting to be such that

1. The surface is above the $\mathbb{S}_{N}$ plane.

2. $P$ is above the surface .

3. In any event we assume that the projection points of $\mathbb{H}$ on the surface belong to a bounded set of $\mathbb{H}_{N}$, then $0 \leq t \leq l<\infty$.

Now we present a definition of a boundization

Definition 3.4.1 A boundization is a mapping that takes an unbounded set into a bounded set in a certain topology.

However, in here we will specifically study boundizations that are induced by nonlinear projection of an Ultra Extended Hilbert space onto surfaces and we will refer to each specific projection induced as boundization.

Definition 3.4.2 a general surface is a mapping $\varphi$ form $\mathbb{H}_{N}$ to $\mathbb{R}$, namely

$$
\varphi(Z)=r \quad Z \in H_{N}
$$

$r$ is fixed.

Definition 3.4.3 A boundization is a mapping of $(\overrightarrow{0}, q) \in \mathbb{U} \mathbb{E} \mathbb{H}$ in a one to one manner on a surface $\varphi(Z)=r$ such that $\overrightarrow{P Z}=t_{g} \overrightarrow{P Q}, 0 \leq t_{g} \leq l<\infty$.

If $P, Z$ and $Q$ lie on the straight line then the vectors $\overrightarrow{P Q}$ and $\overrightarrow{P Z}$ are collinear and impose two conditions the collinearity

$$
\overrightarrow{P Z}=t_{g} \overrightarrow{P Q} \text { where } t_{g} \in \mathbb{R}
$$

requires that, $\left(\left(c-\gamma_{g}\right) u, z\right)=t_{g}\left(-\gamma_{g} u, q\right)$ then

$$
z=t_{g} q \text { and } c=\left(1-t_{g}\right) \gamma_{g}
$$


from $\|\overrightarrow{P Z}\|=t_{g}\|\overrightarrow{P Q}\|$ we get $0 \leq t \leq l$. Here, $t_{g}$ should be viewed as a contraction factor, and should be a real number because when we multiply by a complex number, it's real part gives the scaling and imaginary part rotates it. Here, we need the vectors to be collinear, so we omit the rotation and $t_{g}$ has to be real only. Moreover since $t_{g}$ is a contraction factor then $0 \leq t_{g} \leq l$. From (3.4.3) it is easy to see that the solution of the equation (3.4.1) for $t_{g}$ is always a function of $q$ namely, $t_{g}=t_{g}(\|q\|)$. Since $z$ is bounded then by (3.4.3) we have

$$
t_{g}=\frac{z}{q} \quad \text { and } \quad \lim _{q \longrightarrow \infty U} t_{g}=0
$$

Since $t_{g}$ vanishes as $q \longrightarrow \infty U$. We assume that for some $Z \in \mathbb{H}_{N}$ and $t_{g}$ we have

$$
\lim _{q \longrightarrow \infty U} Z=\lim _{q \longrightarrow \infty U} t_{g} q=\psi(\|q\|) \frac{q}{\|q\|}=\sqrt{-\alpha_{g}} U
$$

$\alpha_{g}$ is a finite non positive real number and $t_{g} q \sim \sqrt{-\alpha_{g}} U$ where $U=\frac{q}{\|q\|}$.

Remark $L$ defined in chapter two in equation (3.1.1) is identical with $\sqrt{-\alpha_{g}}$ that was obtained by geometric consideration.

Assume that $\hat{Z}=(\hat{c} u, \hat{z}) \in \mathbb{H}_{N}$ lies on a surface and $\hat{Q}=(\overrightarrow{0}, \hat{q})$ be an arbitrary points in $\mathbb{U} \mathbb{E} \mathbb{H}$ such that

$$
\overrightarrow{P Z}=\hat{t}_{g} \overrightarrow{P Q} \quad \text { where } \hat{t}_{g} \in \mathbb{R}
$$

requires that, $\left(\left(\hat{c}-\gamma_{g}\right) u, \hat{z}\right)=\hat{t}_{g}\left(-\gamma_{g} u, \hat{q}\right)$ then

$$
\hat{z}=\hat{t}_{g} \hat{q} \text { and } \hat{c}=\left(1-\hat{t}_{g}\right) \gamma_{g} .
$$

Let $Z, Q$ and $t_{g}$ as a sequences in (3.4.6) namely, $Z_{n}=\left(c_{n} u, z_{n}\right), Q_{n}=\left(\overrightarrow{0}, q_{n}\right)$ and $t_{g}=t_{g n}$ so we have

$$
\overrightarrow{P Z_{n}}=t_{g n} \overrightarrow{P Q_{n}}
$$

requires that, $\left(\left(c_{n}-\gamma_{g}\right) u, z_{n}\right)=t_{g n}\left(-\gamma_{g} u, q_{n}\right)$ then

$$
z_{n}=t_{g n} q_{n} \text { and } c_{n}=\left(1-t_{g n}\right) \gamma_{g}
$$

Consider $\hat{Z}, \hat{Q}$ and $\hat{t}_{g}$ as sequences in (3.4.6) namely, $\hat{Z}_{n}=\left(\hat{c}_{n} u, \hat{z}_{n}\right), \hat{Q}_{n}=$ $\left(\overrightarrow{0}, \hat{q}_{n}\right)$ and $\hat{t}_{g}=\hat{t}_{g n}$ so we have

$$
{\overrightarrow{P Z_{n}}}=\hat{t}_{g n} \overrightarrow{P \hat{Q}_{n}}
$$


requires that, $\left(\left(\hat{c}_{n}-\gamma_{g}\right) u, \hat{z}_{n}\right)=\hat{t}_{g n}\left(-\gamma_{s} u, \hat{q}_{n}\right)$ then

$$
\hat{z}_{n}=\hat{t}_{s n} \hat{q}_{n} \text { and } \hat{c}_{n}=\left(1-\hat{t}_{s n}\right) \gamma_{s}
$$

In what follows we present surfaces in Hilbert spaces that are based on conic sections in plane and study geometry.

\subsection{A Sphere Boundization}

A sphere boundization is an example of a general surface boundization and it helps us establish boundization formulas. We consider a sphere with center at a point $O=\left(\theta_{s} u, \overrightarrow{0}\right), \theta_{s} \in \mathbb{C}$ and radius $R \geq 0$. Let $Z \in \mathbb{H}_{N}$ be a point on the sphere $S P\left(\theta_{s}, R\right):=\left\{Z \in \mathbb{H}_{N}:\|Z-O\|_{\mathbb{H}_{N}}=R\right\}$. Fix a "projection point" $P=\left(\gamma_{s} u, \overrightarrow{0}\right), 0 \neq \gamma_{s} \in \mathbb{C}$. We make additional notations and assumptions, the motivation and purpose of which will be clarified by subsequent proceedings.

We assume

I) $\alpha_{s}:=\gamma_{s} \bar{\gamma}_{s}-\gamma_{s} \bar{\theta}_{s}-\theta_{s} \bar{\gamma}_{s}+\theta_{s} \bar{\theta}_{s}-R^{2}=\left|\gamma_{s}-\theta_{s}\right|^{2}-R^{2} \leq 0$.

II) $\left|\theta_{s}\right| \leq\left|\gamma_{s}\right|$.

We add the notation and identity

$$
\mathcal{A}:=-2 \gamma_{s} \bar{\gamma}_{s}+\gamma_{s} \bar{\theta}_{s}+\theta_{s} \bar{\gamma}_{s}=-\left[\left|\gamma_{s}\right|^{2}-\left|\theta_{s}\right|^{2}+\left|\gamma_{s}-\theta_{s}\right|^{2}\right] .
$$

where $\mathcal{A}$ and $\alpha_{s}$ are values that capture the specifies of the geometry of the sphere The assumptions I) and II) above may be replaced by the assumptions III) and IV) below.

III) $\alpha_{s}=\gamma_{s} \bar{\gamma}_{s}-\gamma_{s} \bar{\theta}_{s}-\theta_{s} \bar{\gamma}_{s}+\theta_{s} \bar{\theta}_{s}-R^{2}=\left|\gamma_{s}-\theta_{s}\right|^{2}-R^{2} \leq 0$.

IV) $\mathcal{A} \leq 0$. Condition IV) is recognized to hold iff the values of $\gamma_{s}, \theta_{s}$ satisfy II). II) or IV) hold without loss of generality. If $\mathcal{A}>0$ the formulas to be derived in the sequel need to change form. I) or III) mean that the distance between the center of the sphere $\left(\theta_{s} u, \overrightarrow{0}\right)$ and the location of the fixed projection point $\left(\gamma_{s} u, \overrightarrow{0}\right)$ should not exceed $R$. Then

$$
\alpha_{s}=0 \Longleftrightarrow\left|\gamma_{s}-\theta_{s}\right|^{2}=R^{2} \Longleftrightarrow\left|\gamma_{s}-\theta_{s}\right|=R \text {. }
$$

Namely, the distance between the center of the sphere and the projection point is precisely $R$. We formally recognize this in the important .

Definition 3.5.1 We say that our projection is stereographic if $\alpha=0$. We then call the point $\left(\gamma_{s} u, \overrightarrow{0}\right)$ the north (or south) pole of the sphere. 


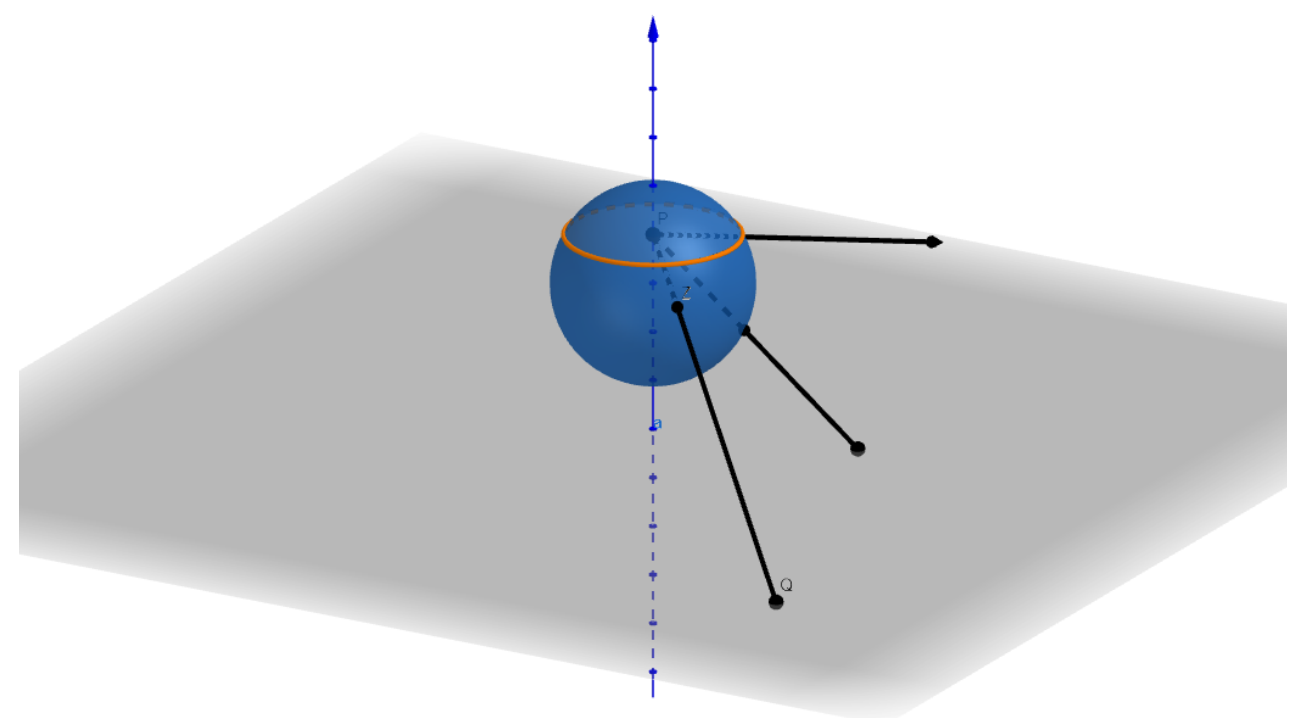

Figure 3.1: Sphere

Condition I) (or III)) may be interpreted geometrically as follows. Let $\mathbb{H}=$ $\mathbb{R}^{2}$ be defined over the field $\mathbb{R}$. Then, $\left|\gamma_{s}-\theta_{s}\right| \leq R \Longleftrightarrow-R \leq \gamma_{s}-\theta_{s} \leq R$ so if $0 \leq \theta_{s}$ then $0 \leq \theta_{s} \leq \gamma_{s} \leq \theta_{s}+R$ and if $\theta_{s} \leq 0$ then $\theta_{s}-R \leq \gamma_{s} \leq \theta_{s} \leq 0$ - Namely, the projection point is situated between the center of the sphere and the north (south) pole. In order to visualize the above geometrical interpretation in a complex Hilbert space we could add the assumption that $\theta_{s}=m \gamma_{s}, 0 \leq m \leq 1$. It should come without surprise that "collinearity" in a complex vector space does not lend itself to intuition as "collinearity" in a real vector space. In what follows we construct a spherical projection mapping $\operatorname{SPM}\left(\theta_{s}, R, \gamma_{s}\right)$ that takes an arbitrary point $Q=(\overrightarrow{0}, q) \in \mathbb{S}_{N}$ into a point $Z=(\mathrm{cu}, z) \in \mathbb{H}_{N}$ that lies on the surface of the sphere $\operatorname{SP}\left(\theta_{s}, R\right)=$ $\left\{Z \in \mathbb{H}_{N}:\|Z-O\|_{H_{N}}=R\right\}$ and we describe its properties. We require the points $P, Z$ and $Q$ to "lie on the same straight line" and to be such that the "arrows" $\overrightarrow{P Z}:=Z-P$ and $\overrightarrow{P Q}:=Q-P$ possess the same direction.

Lemma 3.5.1 i) There exists a unique non negative scalar $0 \leq t_{s} \leq l \in \mathbb{R}$ and $a$ unique point $Z$ on the sphere $S P\left(\theta_{s}, R\right):=\left\{Z \in \mathbb{H}_{N}:\|Z-O\|_{\mathbb{H}_{N}}=R\right\}$ such that

$$
Z-P=t_{s}(Q-P) \text { where } 0 \leq t_{s} \in \mathbb{R} .
$$


The mapping from $\mathbb{S}_{N}$ into the sphere is a bijection. ii) "Circles" $\|(\overrightarrow{0}, q)\|=\rho$ in $\mathbb{S}_{N}$ map onto circles

$$
\|z\|=\hat{\rho}=\frac{-\frac{\mathcal{A}}{2}+\sqrt{\frac{\mathcal{A}^{2}}{4}-\left(\rho^{2}+\gamma_{s} \bar{\gamma}_{s}\right) \alpha}}{\rho^{2}+\gamma_{s} \bar{\gamma}_{s}} \rho \text { in } \mathbb{H}_{N} .
$$

iii) If $\alpha_{s}<0$ the image of the complex Hilbert space $\mathbb{H}$ on the sphere has the shape of an "open bowl" . iv) If $\alpha_{s}=0$ the image of the complex Hilbert space $\mathbb{H}$ is the entire sphere save one point which is the "north pole".

Proof The relation (3.5.3) requires that, $\left(\left(c-\gamma_{s}\right) u, z\right)=t_{s}\left(-\gamma_{s} u, q\right)$ so that

$$
z=t_{s} q \text { and } c=\left(1-t_{s}\right) \gamma_{s} .
$$

The equation of the sphere $\|Z-O\|=R$ in combination with (3.5.4) yields

$$
\langle z, z\rangle+\left\langle\left(c-\theta_{s}\right) u,\left(c-\theta_{s}\right) u\right\rangle=R^{2},
$$

therefore

$$
R^{2}=\left\langle t_{s} q, t_{s} q\right\rangle+\left\langle\left(\left(1-t_{s}\right) \gamma_{s}-\theta_{s}\right) u,\left(\left(1-t_{s}\right) \gamma_{s}-\theta_{s}\right) u\right\rangle .
$$

Recall that $\langle u, u\rangle=1, t_{s}=\bar{t}_{s}$ so we have

$$
R^{2}=t_{s}^{2}\langle q, q\rangle+\left(1-t_{s}\right)^{2} \gamma_{s} \overline{\gamma_{s}}-\left(1-t_{s}\right) \gamma_{s} \bar{\theta}_{s}-\left(1-t_{s}\right) \theta_{s} \overline{\gamma_{s}}+\theta_{s} \bar{\theta}_{s}
$$

and then

$\left.t_{s}^{2}\left\{\langle q, q\rangle+\gamma_{s} \bar{\gamma}_{s}\right\}\right\}+\left\{(-2) \gamma_{s} \bar{\gamma}_{s}+\gamma_{s} \bar{\theta}_{s}+\theta_{s} \bar{\gamma}_{s}\right\} t_{s}-\theta_{s} \bar{\gamma}_{s}-\gamma_{s} \bar{\theta}_{s}+\gamma_{s} \bar{\gamma}_{s}+\theta_{s} \bar{\theta}_{s}-R^{2}=0$.

We identify the quantities that are fixed for the process of boundization. Put

$$
\mathcal{A}=-2 \gamma_{s} \bar{\gamma}_{s}+\gamma_{s} \bar{\theta}_{s}+\theta_{s} \bar{\gamma}_{s}
$$

and

$$
\alpha_{s}=\gamma_{s} \bar{\gamma}_{s}-\gamma_{s} \bar{\theta}_{s}-\theta_{s} \bar{\gamma}_{s}+\theta_{s} \bar{\theta}_{s}-R^{2}=\left(\gamma_{s}-\theta_{s}\right) \overline{\left(\gamma_{s}-\theta_{s}\right)}-R^{2}
$$

Collection and rearranging terms lead to

$$
\left.t_{s}^{2}\left\{\langle q, q\rangle+\gamma_{s} \bar{\gamma}_{s}\right\}\right\}+\mathcal{A} t_{s}+\alpha=0 .
$$


The quadratic equation (3.5.8) has two real roots

$$
t_{s}=\frac{-\frac{\mathcal{A}}{2} \pm \sqrt{F}}{\|q\|^{2}+\gamma_{s} \bar{\gamma}_{s}}, F:=\frac{\mathcal{A}^{2}}{4}-\left(\|q\|^{2}+\gamma_{s} \bar{\gamma}_{s}\right) \alpha_{s}
$$

Note that since $\gamma_{s} \bar{\gamma}_{s}>\theta_{s} \bar{\theta}_{s}$ then by Cauchy Schwartz inequality we get $\mathcal{A} \leq 0$. Since

$$
\sqrt{F}=\sqrt{\frac{\mathcal{A}^{2}}{4}-\left(\langle q, q\rangle+\gamma_{s} \bar{\gamma}_{s}\right) \alpha_{s}} \geq \sqrt{\frac{\mathcal{A}^{2}}{4}}=\frac{|\mathcal{A}|}{2}=\frac{-\mathcal{A}}{2}
$$

then

or

$$
\left|t_{s}\right|=\frac{-\frac{\mathcal{A}}{2}+\sqrt{F}}{\|q\|^{2}+\gamma_{s} \bar{\gamma}_{s}}
$$

$$
\left|t_{s}\right|=\frac{\frac{\mathcal{A}}{2}+\sqrt{F}}{\|q\|^{2}+\gamma_{s} \bar{\gamma}_{s}}
$$

By virtue of the requirement $0 \leq t_{s}$ we must choose the root in (3.5.9) to be

$$
t_{s}=t_{s}(\|q\|)=\frac{-\frac{\mathcal{A}}{2}+\sqrt{F}}{\|q\|^{2}+\gamma_{s} \bar{\gamma}_{s}} .
$$

In sum the point $Q=(\overrightarrow{0}, q) \in \mathbb{S}_{N}$ is matched to the point $Z=\left(\left(1-t_{s}\right) \gamma_{s} u, t_{s} q\right)$ by a spherical projection mapping constructed above. Finally we have

$$
\operatorname{SPM}\left(\theta_{s}, R, \gamma_{s}\right)[(\overrightarrow{0}, q)]=\left(\left(1-t_{s}\right) \gamma_{s} u, t_{s} q\right) .
$$

$\operatorname{SPM}\left(\theta_{s}, R, \gamma_{s}\right)$ is a bijection from $\mathbb{S}_{N}$ onto a subset of the sphere

$$
S P\left(\theta_{s}, R\right):=\left\{Z \in \mathbb{H}_{N} \mid\|Z-O\|_{\mathbb{H}_{N}}=R\right\} .
$$

In order to complete the proof of i) we need to prove that the mapping (3.5.11) is one to one. To that end we notice that

$$
q=t_{s}^{-1} z,\|q\|=t_{s}^{-1}\|z\|, \text { and } t_{s} \neq 0 \Longleftrightarrow\|q\| \neq \infty
$$

and we attempt to express $t_{s}$ in terms of $\|z\|$. The quadratic equation (3.5.8) can be modified to become

$$
\left(\|z\|^{2}+\alpha_{s}\right) t_{s}^{-2}+\mathcal{A} t_{s}^{-1}+\gamma_{s} \bar{\gamma}_{s}=0 .
$$


It is beneficial to discuss separately the stereographic projection case where $\alpha_{s}=0$ as its properties differs radically from the projections when $\alpha_{s}<0$. We notice that with $\alpha_{s}<0$ the product of roots of the quadratic (3.5.13) must be negative because the product of roots of (3.5.8) is negative. Hence, for all $\|z\|^{2}$ in the image $z=t_{s} q$ we must have $\left(\|z\|^{2}+\alpha_{s}\right)<0 \Longleftrightarrow\|z\|^{2}<-\alpha_{s}$ which implies that the positive root of (3.5.13) is given by

$$
t_{s}^{-1}=\frac{-\mathcal{A}-\sqrt{\mathcal{A}^{2}-4 \gamma_{s} \bar{\gamma}_{s}\left(\|z\|^{2}+\alpha_{s}\right)}}{2\left(\|z\|^{2}+\alpha_{s}\right)}=\frac{\frac{\mathcal{A}}{2}+\sqrt{\frac{\mathcal{A}^{2}}{4}-\gamma_{s} \bar{\gamma}_{s}\left(\|z\|^{2}+\alpha_{s}\right)}}{-\left(\|z\|^{2}+\alpha_{s}\right)} .
$$

Thus $q=t_{s}^{-1} z=\frac{-\frac{\mathcal{A}}{2}-\sqrt{\frac{\mathcal{A}^{2}}{4}-\gamma_{s} \bar{\gamma}_{s}\left(\|z\|^{2}+\alpha_{s}\right)}}{\left(\|z\|^{2}+\alpha_{s}\right)} z$ and i) is concluded. For $\alpha_{s}=0$ the quadratic equation is

$t_{s}^{2}\left\{\langle q, q\rangle+\gamma_{s} \bar{\gamma}_{s}\right\}+\mathcal{A} t_{s} \equiv t_{s}\left[\left\{\langle q, q\rangle+\gamma_{s} \bar{\gamma}_{s}\right\} t_{s}+\mathcal{A}\right] \Rightarrow t_{s}=\frac{-\mathcal{A}}{\left\{\|q\|^{2}+\gamma_{s} \bar{\gamma}_{s}\right\}}>0$,

and it has a unique non zero (positive) desired root. In order to obtain the inverse mapping we put

$$
\begin{gathered}
\langle z, z\rangle+t_{s}^{2}\left\{\gamma_{s} \bar{\gamma}_{s}\right\}+\mathcal{A} t_{s}=0 \Longleftrightarrow\langle z, z\rangle t_{s}^{-2}+\mathcal{A} t_{s}^{-1}+\gamma_{s} \bar{\gamma}_{s}=0 . \\
t_{s}=\frac{-\frac{\mathcal{A}}{2}+\sqrt{F}}{\|q\|^{2}+\gamma_{s} \bar{\gamma}_{s}}=\frac{-\alpha_{s}}{\sqrt{F}+\frac{\mathcal{A}}{2}} \quad \text { if } \alpha_{s}<0 .
\end{gathered}
$$

and

$$
t_{s_{0}}=\frac{-\mathcal{A}_{0}}{\|q\|^{2}+\gamma_{s_{0}} \bar{\gamma}_{s_{0}}} \quad \text { if } \alpha_{s}=0
$$

Whilst (3.5.10) is a valid formula for all $\alpha_{s} \leq 0$ the simpler formula (3.5.17) holds for $\alpha_{s}$ strictly negative as for $\alpha_{s}=0$ the expression $t_{s}=\frac{-\alpha_{s}}{\sqrt{F}+\frac{\mathcal{A}}{2}}$ is indeterminate as the denominator is also zero. ii) A property of the stereographic projection that was known to Ptolemy in $\mathbb{R}^{3}$ can be shown to hold in complex Hilbert spaces for any projection $\operatorname{SPM}\left(\theta_{s}, R, \gamma_{s}\right)$. It claims that "circles" $\|\left(\overrightarrow{0, q)}\left\|_{H_{N}}=\right\| q \|_{H}=\rho\right.$ in $\mathbb{S}_{N}$ are mapped under $\operatorname{SPM}\left(\theta_{s}, R, \gamma_{s}\right)$ onto "circles" in $\mathbb{H}_{N}$. Let us verify that this is the case. Indeed, the "plane" $Z=\left(\left(1-t_{s}(\rho)\right) \gamma_{s} u, v\right), v \in \mathbb{H}$, "is parallel" to $\mathbb{S}_{N}$. Its intersection with 
the sphere $\left\{Z \in \mathbb{H}_{N} \mid\|Z-O\|_{H_{N}}=R\right\}$ is a "circle" in $\mathbb{H}_{N}$ with center at $\hat{O}:=\left(\left(1-t_{s}(\rho)\right) \gamma_{s} u, \overrightarrow{0)}\right.$ and radius $r(\rho)$ $r^{2}(\rho)=<Z-\hat{O}, Z-\hat{O}>_{H_{N}}=<\left(\overrightarrow{0}, t_{s}(\rho) q\right),\left(\overrightarrow{0}, t_{s}(\rho) q\right)>_{H_{N}}=t_{s}^{2}(\rho)\|q\|^{2}=\|z\|^{2}$.

The constant value of $r(\rho)$ is readily obtained from the relation

$$
z=t_{s}(\rho) q \Rightarrow r(\rho)=\|z\|=t_{s}(\rho) \rho=\frac{-\frac{\mathcal{A}}{2}+\sqrt{\frac{\mathcal{A}^{2}}{4}-\left(\rho^{2}+\gamma_{s} \bar{\gamma}_{s}\right) \alpha_{s}}}{\rho^{2}+\gamma_{s} \bar{\gamma}_{s}} \rho
$$

This concludes the proof of ii). The "open bowl" shaped image of $\mathbb{S}_{N}$ is obtained by varying $\rho, 0 \leq \rho<\infty$. If $\alpha_{s}<0$ we have from the above that $r(0)=0$ and that $\lim _{\rho \rightarrow \infty} r(\rho)=\sqrt{-\alpha_{s}}>0$ which proves that the "bowl" shaped set is not the entire sphere. In the case that $\alpha_{s}=0$ it is evident that $r(0)=\lim _{\rho \rightarrow \infty} r(\rho)=\sqrt{-\alpha_{s}}=0$ and that the entire sphere is the image of $S_{N}$ save the north pole.

Remark We point out that ((3.5.10)) and (3.5.11) for all $\alpha_{s} \leq 0$. However, the simpler formula for $t_{s}$ prescribed by $((3.5 .17))$ is not valid for $\alpha_{s}=0$.

Remark The "collinear" of three points in a real vector space or the "collinearity" of two vectors in a real vector space is compatible with our geometric interpretation and with our intuition. However, in a vector space over the field of complex numbers $\mathbb{C}$, "collinearity" is not to be interpreted as it is in a real vector space $\mathbb{R}^{n}$ over the field $\mathbb{R}$. Multiplying a vector by a complex number may result in rotation. In a vector space over the Field $\mathbb{C}$ it would be more appropriate to use the language of linear dependence and linear independence.

Let $\hat{Z}=(\hat{c} u, \hat{z}) \in \mathbb{H}_{N}$ lies on a surface and $\hat{Q}=(\overrightarrow{0}, \hat{q})$ be an arbitrary point in $\mathbb{H}_{N}$ such that

$$
\overrightarrow{P Z}=\hat{t}_{s} \overrightarrow{P Q} \quad \text { where } \hat{t}_{s} \in \mathbb{R}
$$

requires that, $\left(\left(\hat{c}-\gamma_{s}\right) u, \hat{z}\right)=\hat{t}_{s}\left(-\gamma_{s} u, \hat{q}\right)$ then

$$
\hat{z}=\hat{t}_{s} \hat{q} \text { and } \hat{c}=\left(1-\hat{t}_{s}\right) \gamma_{s}
$$

Now consider $Z, Q$ and $t_{s}$ as a sequences in

$$
\overrightarrow{P Z}=\hat{t}_{s} \overrightarrow{P Q} \quad \text { where } \hat{t}_{s} \in \mathbb{R}
$$


as follows $Z_{n}=\left(c_{n} u, z_{n}\right), Q_{n}=\left(\overrightarrow{0}, q_{n}\right)$ and $t_{s}=t_{\text {sn }}$ so we have

$$
\overrightarrow{P Z_{n}}=t_{s n} \overrightarrow{P Q_{n}}
$$

requires that, $\left(\left(c_{n}-\gamma_{s}\right) u, z_{n}\right)=t_{s n}\left(-\gamma_{s} u, q_{n}\right)$ then

$$
z_{n}=t_{s n} q_{n} \text { and } c_{n}=\left(1-t_{s n}\right) \gamma_{s}
$$

Also consider $\hat{Z}, \hat{Q}$ and $\hat{t}_{s}$ as a sequences in (3.5.20) as follows $\hat{Z}_{n}=\left(\hat{c}_{n} u, \hat{z}_{n}\right)$, $\hat{Q}_{n}=\left(\overrightarrow{0}, \hat{q}_{n}\right)$ and $\hat{t}_{s}=\hat{t}_{s n}$ so we have

$$
\vec{P}_{n}=\hat{t}_{s n} \overrightarrow{P \hat{Q}_{n}}
$$

requires that, $\left(\left(\hat{c}_{n}-\gamma_{s}\right) u, \hat{z}_{n}\right)=\hat{t}_{s n}\left(-\gamma_{s} u, \hat{q}_{n}\right)$ then

$$
\hat{z}_{n}=\hat{t}_{s n} \hat{q}_{n} \text { and } \hat{c}_{n}=\left(1-\hat{t}_{s n}\right) \gamma_{s}
$$

Repeat the steps above with the following points $\hat{Z}=(\hat{c} u, \hat{z})$ and $\hat{Q}=(0, \hat{q})$, also consider $\|\overrightarrow{O Z}\|_{H_{N}}^{2}=R^{2}$, then by similar arguments we get

$$
\hat{t}_{s}=\frac{-\frac{\mathcal{A}}{2}+\sqrt{\hat{F}}}{\|\hat{q}\|^{2}+\gamma_{s} \bar{\gamma}_{s}}
$$

where

$$
\hat{F}=\frac{\mathcal{A}^{2}}{4}-\left(\|\hat{q}\|^{2}+\gamma_{s} \bar{\gamma}_{s}\right) \alpha_{s}
$$

Proposition 3.5.2 if $0 \neq\left\|q_{n}\right\| \longrightarrow \infty$ as $n \longrightarrow \infty$ then $t_{s n} \longrightarrow 0$ as $n \longrightarrow \infty$

Proof Since

$$
t_{s n}=\frac{-\frac{\mathcal{A}}{2}+\sqrt{\frac{\mathcal{A}^{2}}{4}-\left(\left\|q_{n}\right\|^{2}+\gamma_{s} \bar{\gamma}_{s}\right) \alpha_{s}}}{\left\|q_{n}\right\|^{2}+\gamma_{s} \bar{\gamma}_{s}}
$$

we divide both the numerator and denominator of (3.5.28) by $\left\|q_{n}\right\|^{2}$ we get

$$
t_{s n}=\frac{-\frac{\mathcal{A}}{2\left\|q_{n}\right\|^{2}}+\sqrt{\frac{\mathcal{A}^{2}}{4\left\|q_{n}\right\|^{4}}-\left(\frac{1}{\left\|q_{n}\right\|^{2}}+\frac{\gamma_{s} \bar{\gamma}_{s}}{\left\|q_{n}\right\|^{4}}\right) \alpha_{s}}}{1+\frac{\gamma_{s} \bar{\gamma}_{s}}{\left\|q_{n}\right\|^{2}}}
$$

when $\left\|q_{n}\right\| \longrightarrow \infty$ we get $t_{s n} \longrightarrow 0$ as $n \longrightarrow \infty$ 
Proposition 3.5.3 Consider a sequences $t_{\text {sn }}$ and $q_{n} \neq 0$ subject to (3.5) then for $\alpha_{s}<0$ we have

$$
t_{s n} \sim \frac{\sqrt{-\alpha_{s}}}{\left\|q_{n}\right\|} \text { as } n \longrightarrow \infty
$$

and

$$
t_{s n} q_{n} \sim \sqrt{-\alpha_{s}} U_{n} \quad \text { as } n \longrightarrow \infty
$$

where

$$
U_{n}=\frac{q_{n}}{\left\|q_{n}\right\|}
$$

Proof Since $\alpha_{s}<0$ then we have

$$
F_{n}=\frac{\mathcal{A}^{2}}{4}-\left\|q_{n}\right\|^{2} \alpha_{s}-\gamma_{s} \bar{\gamma}_{s} \alpha_{s}=-\left\|q_{n}\right\|^{2} \alpha_{s}\left[1-\frac{\frac{\mathcal{A}^{2}}{4}-\gamma_{s} \bar{\gamma}_{s} \alpha_{s}}{\left\|q_{n}\right\|^{2} \alpha_{s}}\right]
$$

Thus

$$
\sqrt{F_{n}} \sim\left\|q_{n}\right\| \sqrt{-\alpha_{s}} \text { as } n \longrightarrow \infty
$$

therefore

$$
t_{s n} \sim \frac{\frac{\mathcal{A}}{2}+\left\|q_{n}\right\| \sqrt{-\alpha_{s}}}{\left\|q_{n}\right\|^{2}+\gamma_{s} \bar{\gamma}_{s}} \sim \frac{\sqrt{-\alpha_{s}}}{\left\|q_{n}\right\|} \text { as } n \longrightarrow \infty
$$

Also

$$
t_{s n} q_{n} \sim \frac{\sqrt{-\alpha_{s}}}{\left\|q_{n}\right\|} q_{n}=\sqrt{-\alpha_{s}} U_{n} \text { as } n \longrightarrow \infty
$$

Proposition 3.5.4 Consider a sequences $\hat{t}_{\text {sn }}$ and $\hat{q}_{n} \neq 0$ subject to (3.5.26) then for $\alpha_{s}<0$ we have

$$
\hat{t}_{s n} \sim \frac{\sqrt{-\alpha_{s}}}{\left\|\hat{q}_{n}\right\|} \text { as } n \longrightarrow \infty
$$

and

$$
\hat{t}_{s n} \hat{q}_{n} \sim \sqrt{-\alpha_{s}} \hat{U}_{n} \quad \text { as } n \longrightarrow \infty
$$

where

$$
\hat{U}_{n}=\frac{\hat{q}_{n}}{\left\|\hat{q}_{n}\right\|}
$$

Notice that $t_{s n} \longrightarrow 0$ and $\hat{t}_{s n} \longrightarrow 0$ when $n \longrightarrow \infty$. 


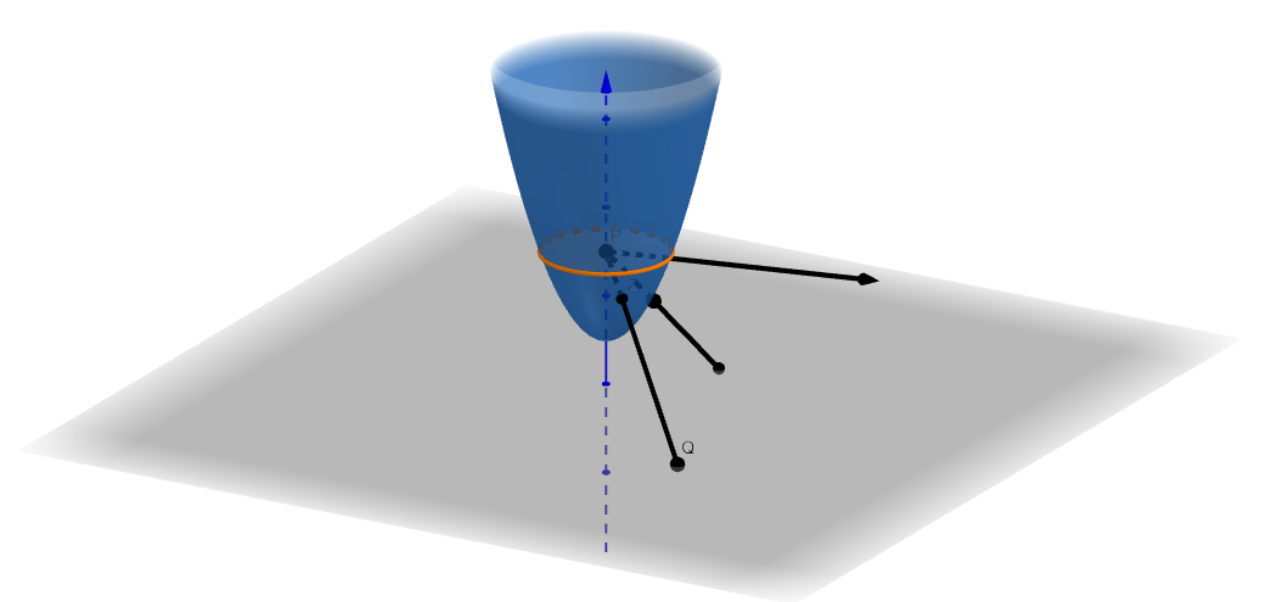

Figure 3.2: Paraboloid

\subsection{A Paraboloid Boundization}

A paraboloid boundization is our second example of a general surface boundization gives us a different formula with different properties then the sphere boundization. As we know a Paraboloid is the set of points in the space that are equidistant from a point (the focus) and a line the (directrix). Let $P=\left(\gamma_{p} u, \overrightarrow{0}\right)$ be a fixed point in $\mathbb{H}_{N}$ where $\gamma_{p} \in \mathbb{C}$ is a fixed number and $Q=(\overrightarrow{0}, q)$ be an arbitrary point in $\mathbb{H}_{N}$ and let $Z=(c u, z) \in \mathbb{H}_{N}$ lies on a surface of a paraboloid. If $P, Z$ and $Q$ lie on the straight line then the vectors $\overrightarrow{P Q}$ and $\overrightarrow{P Z}$ are collinear and impose two conditions

$$
\overrightarrow{P Z}=t_{p} \overrightarrow{P Q} \text { where } t_{p} \in \mathbb{R}
$$

requires that, $\left(\left(c-\gamma_{p}\right) u, z\right)=t_{p}\left(-\gamma_{p} u, q\right)$ then

$$
z=t_{p} q \text { and } c=\left(1-t_{p}\right) \gamma_{p}
$$

Let $\hat{Z}=(\hat{c} u, \hat{z}) \in \mathbb{H}_{N}$ lies on a surface and $\hat{Q}=(\overrightarrow{0}, \hat{q})$ be an arbitrary points in $\mathbb{H}_{N}$ such that

$$
\overrightarrow{P Z}=\hat{t}_{p} \overrightarrow{P Q} \quad \text { where } \hat{t}_{p} \in \mathbb{R}
$$


requires that, $\left(\left(\hat{c}-\gamma_{p}\right) u, \hat{z}\right)=\hat{t}_{p}\left(-\gamma_{p} u, \hat{q}\right)$ then

$$
\hat{z}=\hat{t}_{p} \hat{q} \text { and } \hat{c}=\left(1-\hat{t}_{p}\right) \gamma_{p}
$$

Now consider $Z, Q$ and $t_{p}$ as a sequences in (3.6.1) as follows $Z_{n}=\left(c_{n} u, z_{n}\right)$, $Q_{n}=\left(\overrightarrow{0}, q_{n}\right)$ and $t_{p}=t_{p n}$ so we have

$$
\overrightarrow{P Z_{n}}=t_{p n} \overrightarrow{P Q_{n}}
$$

requires that, $\left(\left(c_{n}-\gamma_{p}\right) u, z_{n}\right)=t_{p n}\left(-\gamma_{p} u, q_{n}\right)$ then

$$
z_{n}=t_{p n} q_{n} \text { and } c_{n}=\left(1-t_{p n}\right) \gamma_{p}
$$

Also consider $\hat{Z}, \hat{Q}$ and $\hat{t}_{p}$ as a sequences in (3.6.3) as follows $\hat{Z}_{n}=\left(\hat{c}_{n} u, \hat{z}_{n}\right)$, $\hat{Q}_{n}=\left(0, \hat{q}_{n}\right)$ and $\hat{t}_{p}=\hat{t}_{p n}$ so we have

$$
{\overrightarrow{P Z_{n}}}=\hat{t}_{p n}{\overrightarrow{P \hat{Q}_{n}}}
$$

requires that, $\left(\left(\hat{c}_{n}-\gamma_{p}\right) u, \hat{z}_{n}\right)=\hat{t}_{p n}\left(-\gamma_{p} u, \hat{q}_{n}\right)$ then

$$
\hat{z}_{n}=\hat{t}_{p n} \hat{q}_{n} \text { and } \hat{c}_{n}=\left(1-\hat{t}_{p n}\right) \gamma_{p}
$$

To develop an equation for a parabola, given the vertex point $(y u, \overrightarrow{0})$, the focus point $\left(\theta_{p} u, \overrightarrow{0}\right)$ and the directrix line $L=\left(2 y-\theta_{p}\right) u$ where $\theta_{p}, y \in \mathbb{C}$. For an arbitrary point $Z=(c u, z) \in \mathbb{H}_{N}$ on the surface of the parabola the distance to the directrix is the distance from $Z=(c u, z)$ to $\left(\left(2 y-\theta_{p}\right) u, z\right)$ namely,

$$
\begin{aligned}
& \left\|(c u, z)-\left(\left(2 y-\theta_{p}\right) u, z\right)\right\|_{H_{N}} \\
& =\left\|\left(\left(c-2 y+\theta_{p}\right) u, \overrightarrow{0}\right)\right\|_{H_{N}} \\
& =\sqrt{\left\langle\left(\left(c-2 y+\theta_{p}\right) u, \overrightarrow{0}\right),\left(\left(c-2 y+\theta_{p}\right) u, \overrightarrow{0}\right)\right\rangle_{H_{N}}} \\
& =\sqrt{\left\langle\left(c-2 y+\theta_{p}\right) u,\left(c-2 y+\theta_{p}\right) u\right\rangle+\langle\overrightarrow{0}, \overrightarrow{0}\rangle} \\
& =\sqrt{\left(c-2 y+\theta_{p}\right) \overline{\left(c-2 y+\theta_{p}\right)}}\langle u, u\rangle \\
& =\sqrt{\left(c-2 y+\theta_{p}\right) \overline{\left(c-2 y+\theta_{p}\right)}}
\end{aligned}
$$


The distance to the focus is the distance between $Z=(c u, z)$ and $\left(\theta_{p} u, \overrightarrow{0}\right)$ is

$$
\begin{aligned}
\left\|(c u, z)-\left(\theta_{p} u, 0\right)\right\|_{H_{N}} & =\left\|\left(\left(c-\theta_{p}\right) u, z\right)\right\|_{H_{N}} \\
& =\sqrt{\left\langle\left(\left(c-\theta_{p}\right) u, z\right),\left(\left(c-\theta_{p}\right) u, z\right)\right\rangle_{H_{N}}} \\
& =\sqrt{\left\langle\left(c-\theta_{p}\right) u,\left(c-\theta_{p}\right) u\right\rangle+\langle z, z\rangle} \\
& =\sqrt{\left(c-\theta_{p}\right) \overline{\left(c-\theta_{p}\right)}\langle u, u\rangle+\|z\|^{2}} \\
& =\sqrt{\left(c-\theta_{p}\right) \overline{\left(c-\theta_{p}\right)}+\|z\|^{2}}
\end{aligned}
$$

We use the fact that these distances are equal to write the equation of the parabola as follows

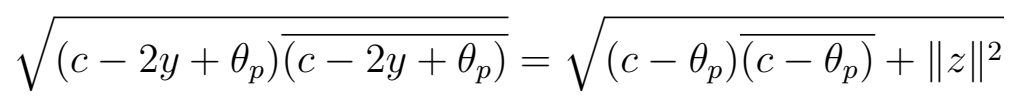

Square each side to get.

$$
\left(c-2 y+\theta_{p}\right) \overline{\left(c-2 y+\theta_{p}\right)}=\left(c-\theta_{p}\right) \overline{\left(c-\theta_{p}\right)}+\|z\|^{2}
$$

therefore

$$
\left(c-2 y+\theta_{p}\right) \overline{\left(c-2 y+\theta_{p}\right)}-\left(c-\theta_{p}\right) \overline{\left(c-\theta_{p}\right)}-\|z\|^{2}=0
$$

To simplify the equation (3.6.9), first remove the parentheses

$$
\begin{aligned}
& \left(c-2 y+\theta_{p} \overline{\left(c-2 y+\theta_{p}\right)}-\left(c-\theta_{p} \overline{\left(c-\theta_{p}\right)}-\|z\|^{2}\right.\right. \\
& =c \bar{c}-2 c \bar{y}+c \bar{\theta}_{p}-2 y \bar{c}+4 y \bar{y}-2 y \bar{\theta}_{p}+\theta_{p} \bar{c}-2 \theta_{p} \bar{y}+\theta_{p} \bar{\theta}_{p} \\
& -c \bar{c}+c \bar{\theta}_{p}+\theta_{p} \bar{c}-\theta_{p} \bar{\theta}_{p}-\|z\|^{2} \\
& =-2 c \bar{y}+2 c \bar{\theta}_{p}-2 y \bar{c}+4 y \bar{y}-2 y \bar{\theta}_{p}+2 \theta_{p} \bar{c}-2 \theta_{p} \bar{y}-\|z\|^{2}=0 \\
& c \bar{\theta}_{p}-c \bar{y}-y \bar{c}+2 y \bar{y}-y \bar{\theta}_{p}+\theta_{p} \bar{c}-\theta_{p} \bar{y}-\frac{\|z\|^{2}}{2}=0
\end{aligned}
$$


Combining (3.6.10) with $c=\left(1-t_{p}\right) \gamma_{p}$ we have

$$
\begin{aligned}
& c \bar{\theta}_{p}-c \bar{y}-y \bar{c}+2 y \bar{y}-y \bar{\theta}_{p}+\theta_{p} \bar{c}-\theta_{p} \bar{y}-\frac{\|z\|^{2}}{2} \\
& =\left(1-t_{p}\right) \gamma_{p} \bar{\theta}_{p}-\left(1-t_{p}\right) \gamma_{p} \bar{y}-y \overline{\left(1-t_{p}\right) \gamma_{p}} \\
& +2 y \bar{y}-y \bar{\theta}_{p}+\theta_{p} \overline{\left(1-t_{p}\right) \gamma_{p}}-\theta_{p} \bar{y}-\frac{\|z\|^{2}}{2} \\
& =\left(1-t_{p}\right) \gamma_{p} \bar{\theta}_{p}-\left(1-t_{p}\right) \gamma_{p} \bar{y}-\left(1-t_{p}\right) y \bar{\gamma}_{p} \\
& +2 y \bar{y}-y \bar{\theta}_{p}+\left(1-t_{p}\right) \theta_{p} \bar{\gamma}_{p}-\theta_{p} \bar{y}-\frac{\|z\|^{2}}{2} \\
& =\gamma_{p} \bar{\theta}_{p}-t_{p} \gamma_{p} \bar{\theta}_{p}-\gamma_{p} \bar{y}+t_{p} \gamma_{p} \bar{y}-y \bar{\gamma}_{p}+t_{p} y \bar{\gamma}_{p} \\
& +2 y \bar{y}-y \bar{\theta}_{p}+\theta_{p} \bar{\gamma}_{p}-t_{p} \theta_{p} \bar{\gamma}_{p}-\theta_{p} \bar{y}-\frac{\|z\|^{2}}{2} \\
& =t_{p}\left(y \bar{\gamma}_{p}+\gamma_{p} \bar{y}-\theta_{p} \bar{\gamma}_{p}-\gamma_{p} \bar{\theta}\right)_{p}-\gamma_{p} \bar{y}-y \bar{\gamma}_{p} \\
& +2 y \bar{y}-y \bar{\theta}_{p}+\theta_{p} \bar{\gamma}_{p}+\gamma_{p} \bar{\theta}_{p}-\theta_{p} \bar{y}-\frac{\|z\|^{2}}{2} \\
& =t_{p}\left(\bar{\gamma}_{p}\left(y-\theta_{p}\right)+\gamma_{p} \overline{\left(y-\theta_{p}\right)}\right)+\bar{\gamma}_{p}\left(\theta_{p}-y\right)+\gamma_{p} \overline{\left(\theta_{p}-y\right)} \\
& +2 y \bar{y}-\left(y \bar{\theta}_{p}+\theta_{p} \bar{y}\right)-\frac{\|z\|^{2}}{2}=0
\end{aligned}
$$

Thus

$$
\begin{aligned}
& \frac{\|z\|^{2}}{2}-t_{p}\left(\bar{\gamma}_{p}\left(y-\theta_{p}\right)+\gamma_{p} \overline{\left(y-\theta_{p}\right)}\right)-\bar{\gamma}_{p}\left(\theta_{p}-y\right)-\gamma_{p} \overline{\left(\theta_{p}-y\right)}-2 y \bar{y}+\left(y \bar{\theta}_{p}+\theta_{p} \bar{y}\right)=0 \\
& \text { or } \\
& \frac{\|z\|^{2}}{2}+t_{p}\left(\bar{\gamma}_{p}\left(\theta_{p}-y\right)+\gamma_{p} \overline{\left(\theta_{p}-y\right)}\right)-\bar{\gamma}_{p}\left(\theta_{p}-y\right)-\gamma_{p} \overline{\left(\theta_{p}-y\right)}-2 y \bar{y}+\left(y \bar{\theta}_{p}+\theta_{p} \bar{y}\right)=0
\end{aligned}
$$

We identify the quantities that are fixed for the process of boundization. Put

$$
\begin{gathered}
\mathcal{B}=2\left(\bar{\gamma}_{p}\left(\theta_{p}-y\right)+\gamma_{p} \overline{\left(\theta_{p}-y\right)}\right) \\
\alpha_{p}=-2 \bar{\gamma}_{p}\left(\theta_{p}-y\right)-2 \gamma_{p} \overline{\left(\theta_{p}-y\right)}-4 y \bar{y}+2\left(y \bar{\theta}_{p}+\theta_{p} \bar{y}\right)=-\mathcal{B}-4 y \bar{y}+2\left(y \bar{\theta}_{p}+\theta_{p} \bar{y}\right)
\end{gathered}
$$

note that $\mathcal{B}>0$ and $\alpha_{p}<0$, therefore we have

$$
\|z\|^{2}+\mathcal{B} t_{p}+\alpha_{p}=0
$$


solve an equation (3.6.13) for $t_{p}$ we get

$$
\begin{aligned}
t_{p} & =\frac{\alpha_{p}+\|z\|^{2}}{-\mathcal{B}}=\frac{-\mathcal{B}-4 y \bar{y}+2\left(y \bar{\theta}_{p}+\theta_{p} \bar{y}\right)+\|z\|^{2}}{-\mathcal{B}} \\
& =1+\frac{-4 y \bar{y}+2\left(y \bar{\theta}_{p}+\theta_{p} \bar{y}\right)+\|z\|^{2}}{-\mathcal{B}} \\
& =1-\frac{-4 y \bar{y}+2\left(y \bar{\theta}_{p}+\theta_{p} \bar{y}\right)+\|z\|^{2}}{\mathcal{B}} \\
& =1-\frac{\alpha_{p}-\mathcal{B}+\|z\|^{2}}{\mathcal{B}}
\end{aligned}
$$

We want to express $t_{p}$ as a function of $q$, from (3.6.2) we obtain

$$
\|z\|^{2}=\langle z, z\rangle=\left\langle t_{p} q, t_{p} q\right\rangle=t_{p}^{2}\langle q, q\rangle=t_{p}^{2}\|q\|^{2}, \quad\|z\|=\left|t_{p}\right|\|q\|
$$

by (3.6.15) we have

$$
t^{2}\|q\|^{2}+\mathcal{B} t_{p}+\alpha_{p}=0
$$

Solving the quadratic equation (3.6.16) for $t_{p}$ we have if

$$
1-\frac{\alpha_{p}-\mathcal{B}+\|z\|^{2}}{\mathcal{B}} \geq 0
$$

then

$$
t_{p}=\frac{-\mathcal{B}+\sqrt{\mathcal{B}^{2}-4\|q\|^{2} \alpha_{p}}}{2\|q\|^{2}}=\frac{\frac{-\mathcal{B}}{2}+\sqrt{\left(\frac{\mathcal{B}}{2}\right)^{2}-\|q\|^{2} \alpha_{p}}}{\|q\|^{2}}
$$

we want to rationalizing the numerator so we will use a simple trick worth remembering, multiply the numerator and the denominator by conjugate and 
simplify to get .

$$
\begin{aligned}
t_{p} & =\frac{\sqrt{\left(\frac{\mathcal{B}}{2}\right)^{2}-\|q\|^{2} \alpha_{p}}-\frac{\mathcal{B}}{2}}{\|q\|^{2}}\left(\frac{\sqrt{\left(\frac{\mathcal{B}}{2}\right)^{2}-\|q\|^{2} \alpha_{p}}+\frac{\mathcal{B}}{2}}{\sqrt{\left(\frac{\mathcal{B}}{2}\right)^{2}-\|q\|^{2} \alpha_{p}}+\frac{\mathcal{B}}{2}}\right) \\
= & \frac{\left(\frac{\mathcal{B}}{2}\right)^{2}-\|q\|^{2} \alpha_{p}-\left(\frac{\mathcal{B}}{2}\right)^{2}}{\|q\|^{2}\left(\frac{\mathcal{B}}{2}+\sqrt{\left(\frac{\mathcal{B}}{2}\right)^{2}-\|q\|^{2} \alpha_{p}}\right)} \\
= & \frac{-\|q\|^{2} \alpha_{p}}{\|q\|^{2}\left(\frac{\mathcal{B}}{2}+\sqrt{\left(\frac{\mathcal{B}}{2}\right)^{2}-\|q\|^{2} \alpha_{p}}\right)}=\frac{-\alpha_{p}}{\frac{\mathcal{B}}{2}+\sqrt{\left(\frac{\mathcal{B}}{2}\right)^{2}-\|q\|^{2} \alpha_{p}}} \\
= & \frac{-\alpha_{p}}{\frac{-2 \alpha_{p}}{\mathcal{B}}\left(1+\sqrt{1-\frac{4\|q\|^{2} \alpha_{p}}{\mathcal{B}^{2}}}\right)}=\frac{1+\sqrt{1-\frac{4\|q\|^{2} \alpha_{p}}{\mathcal{B}^{2}}}}{1+\sqrt{\frac{1}{2}}}
\end{aligned}
$$

if

$$
1-\frac{\alpha_{p}-\mathcal{B}+\|z\|^{2}}{\mathcal{B}} \leq 0
$$

then

$$
t_{p}=\frac{\frac{-\mathcal{B}}{2}-\sqrt{\left(\frac{\mathcal{B}}{2}\right)^{2}-\|q\|^{2} \alpha_{p}}}{\|q\|^{2}}
$$

we always choose

$$
t_{p}=\frac{\frac{-2 \alpha_{p}}{\mathcal{B}}}{1+\sqrt{1-\frac{4\|q\|^{2} \alpha_{p}}{\mathcal{B}^{2}}}}
$$

we can write $t_{p}$ as

$$
t_{p}=\frac{-2 \alpha_{p}}{\mathcal{B}+\mathcal{B} \sqrt{G}}
$$

where

$$
G=1-\frac{4\|q\|^{2} \alpha_{p}}{\mathcal{B}^{2}}
$$

The formula (3.6.18) enables us to write $Z=(c u, z)$, on the parabolic bowl, as follows

$$
z=\frac{-2 \alpha_{p} q}{\mathcal{B}+\mathcal{B} \sqrt{G}}
$$




$$
c=\left(1-t_{p}\right) \gamma_{p}=\left(1-\frac{-2 \alpha_{p}}{\mathcal{B}+\mathcal{B} \sqrt{G}}\right) \gamma_{p}=\gamma_{p}\left(\frac{\mathcal{B}+\mathcal{B} \sqrt{G}+2 \alpha_{p}}{\mathcal{B}+\mathcal{B} \sqrt{G}}\right)
$$

we also have via $\|z\|=\left|t_{p}\right|\|q\|$ in (3.6.15)

$$
\|z\|^{2}=\left(\frac{-2 \alpha_{p}}{\mathcal{B}+\mathcal{B} \sqrt{G}}\right)^{2}\|q\|^{2}, \quad\|z\|=\frac{-2 \alpha_{p}\|q\|}{\mathcal{B}+\mathcal{B} \sqrt{G}}
$$

From the relation

$$
t_{p}=1-\frac{\alpha_{p}-\mathcal{B}+\|z\|^{2}}{\mathcal{B}}
$$

we can obtain the inverse relations

$$
q=\frac{z}{t_{p}}=\frac{z}{1-\frac{\alpha_{p}-\mathcal{B}+\|z\|^{2}}{\mathcal{B}}}=\frac{\mathcal{B} z}{\mathcal{B}-\left(\alpha_{p}-\mathcal{B}\right)+\|z\|^{2}}
$$

Consider $z_{n}$ subject to (3.6.6) then we have

$$
\begin{aligned}
& z_{n}=\frac{-2 \alpha_{p} q_{n}}{\mathcal{B}+\mathcal{B} \sqrt{G}}=\frac{-2 \alpha_{p}}{\mathcal{B}+\mathcal{B} \sqrt{1-\frac{4\|q\|^{2} \alpha_{p}}{\mathcal{B}^{2}}}} \\
& =\frac{-2 \alpha_{p} q_{n}}{\mathcal{B}+\mathcal{B} \sqrt{-\frac{4\left\|q_{n}\right\|^{2} \alpha_{p}}{\mathcal{B}^{2}}\left(-\frac{\mathcal{B}^{2}}{4\left\|q_{n}\right\|^{2} \alpha_{p}}+1\right)}} \\
& =\frac{-2 \alpha_{p} q_{n}}{\mathcal{B}+2\left\|q_{n}\right\| \sqrt{-\alpha_{p}} \sqrt{-\frac{\mathcal{B}^{2}}{4\left\|q_{n}\right\|^{2} \alpha_{p}}+1}} \\
& =\left(\frac{q_{n}}{\left\|q_{n}\right\|}\right)\left(\frac{\sqrt{-\alpha_{p}}}{\frac{\mathcal{B}}{2\left\|q_{n}\right\| \sqrt{-\alpha_{p}}}+\sqrt{-\frac{\mathcal{B}^{2}}{4\left\|q_{n}\right\|^{2} \alpha_{p}}+1}}\right)
\end{aligned}
$$

Let $\hat{Z}=(\hat{c} u, \hat{z})$ lies on a parabolic surface and consider $\hat{t}_{p}$ subject to (3.6.3) then we have

$$
\begin{aligned}
\hat{t}_{p} & =1-\frac{\alpha_{p}-\mathcal{B}+\|\hat{z}\|^{2}}{\mathcal{B}} \\
& = \begin{cases}\frac{-2 \alpha_{p} q_{n}}{\mathcal{B}+\mathcal{B} \sqrt{\hat{G}}} & \text { if } 1-\frac{\alpha_{p}-\mathcal{B}+\|\hat{z}\|^{2}}{\mathcal{B}} \geq 0 \\
\frac{\frac{-\mathcal{B}}{2}-\sqrt{\left(\frac{\mathcal{B}}{2}\right)^{2}-\|\hat{q}\|^{2} \alpha_{p}}}{\|\hat{q}\|^{2}} & \text { if } 1-\frac{\alpha_{p}-\mathcal{B}+\|\hat{z}\|^{2}}{\mathcal{B}} \leq 0\end{cases}
\end{aligned}
$$


we always choose

$$
\hat{t}_{p}=\frac{-2 \alpha_{p}}{\mathcal{B}+\mathcal{B} \sqrt{\hat{G}}}
$$

where

$$
\hat{G}=1-\frac{4\|\hat{q}\|^{2} \alpha_{p}}{\mathcal{B}^{2}}
$$

Proposition 3.6.1 Consider $t_{p}$ subject to (3.6.18) and assume $\rho_{2}>\rho_{1}$ with $\|q\| \leq \rho_{1}$ and $\|q\| \geq \rho_{2}$ then we have

$$
0<\mathcal{N}_{0} \leq t_{p} \leq \frac{-\alpha_{p}}{\mathcal{B}}
$$

where

$$
\mathcal{N}_{0}=\frac{-2 \alpha_{p}}{\mathcal{B}+\mathcal{B} \sqrt{1-\frac{4 \rho_{1}^{2} \alpha_{p}}{\mathcal{B}^{2}}}}
$$

Moreover, $t_{p} \longrightarrow 0$ as $\|q\| \longrightarrow \infty$

Proof For $\|q\| \leq \rho_{1}$ we have

$$
t_{p}=\frac{-2 \alpha_{p}}{\mathcal{B}+\mathcal{B} \sqrt{1-\frac{4\|q\|^{2} \alpha_{p}}{\mathcal{B}^{2}}}} \geq \frac{-2 \alpha_{p}}{\mathcal{B}+\mathcal{B} \sqrt{1-\frac{4 \rho_{1}^{2} \alpha_{p}}{\mathcal{B}^{2}}}}=\mathcal{N}_{0}
$$

For $\|q\| \geq \rho_{2}$ we have

$$
t_{p}=\frac{-2 \alpha_{p}}{\mathcal{B}+\mathcal{B} \sqrt{1-\frac{4\|q\|^{2} \alpha_{p}}{\mathcal{B}^{2}}}} \leq \frac{-2 \alpha_{p}}{2 \mathcal{B}}=\frac{-\alpha_{p}}{\mathcal{B}}
$$

therefore

$$
0<\mathcal{N}_{0} \leq t_{p} \leq \frac{-\alpha_{p}}{\mathcal{B}}
$$

Consider $\hat{z}_{n}$ subject to (3.6.8) then for $\hat{q}_{n} \neq 0$ we have

$$
\hat{z}_{n}=\left(\frac{\hat{q_{n}}}{\| \hat{q_{n} \|}}\right)\left(\frac{\sqrt{-\alpha_{p}}}{\frac{\mathcal{B}}{2\left\|\hat{q}_{n}\right\| \sqrt{-\alpha_{p}}}+\sqrt{-\frac{\mathcal{B}^{2}}{4\left\|\hat{q}_{n}\right\|^{2} \alpha_{p}}+1}}\right)
$$


Proposition 3.6.2 Consider a sequences $t_{p n}$ and $q_{n} \neq 0$ subject to (3.6.6) then we have

$$
t_{p n} \sim \frac{\sqrt{-\alpha_{p}}}{\left\|q_{n}\right\|} \text { as } n \longrightarrow \infty
$$

and the coefficient of $\frac{q_{n}}{\left\|q_{n}\right\|}=U_{n}$ in (3.6.21) tends to $\sqrt{-\alpha_{p}}$ as $n \longrightarrow \infty$

\section{Proof}

$$
\begin{aligned}
t_{p n} & =\frac{-2 \alpha_{p}}{\mathcal{B}+\mathcal{B} \sqrt{1-\frac{4\left\|q_{n}\right\|^{2} \alpha_{p}}{\mathcal{B}^{2}}}} \\
& =\frac{\sqrt{-\alpha_{p}}}{\left\|q_{n}\right\|\left(\frac{\mathcal{B}}{2\left\|q_{n}\right\| \sqrt{-\alpha_{p}}}+\sqrt{-\frac{\mathcal{B}^{2}}{4\left\|q_{n}\right\|^{2} \alpha_{p}}+1}\right)}
\end{aligned}
$$

as $n \longrightarrow \infty$ we obtain $t_{p n} \sim \frac{\sqrt{-\alpha_{p}}}{\left\|q_{n}\right\|}$

Proposition 3.6.3 Consider a sequences $\hat{t}_{p n}$ and $\hat{q}_{n} \neq 0$ subject to (3.6.8) then we have

$$
\hat{t}_{p n} \sim \frac{\sqrt{-\alpha_{p}}}{\left\|\hat{q}_{n}\right\|} \text { as } n \longrightarrow \infty
$$

and the coefficient of $\frac{\hat{q_{n}}}{\left\|\hat{q_{n}}\right\|}=\hat{U}_{n}$ in (3.6.25) tends to $\sqrt{-\alpha_{p}}$ as $n \longrightarrow \infty$.

Notice that $t_{p n} \longrightarrow 0$ and $\hat{t}_{p n} \longrightarrow 0$ when $n \longrightarrow \infty$.

\subsection{A Cone Boundization}

Now, we come to my third example of a general surface boundization, that is called cone boundization. It is more commonly used then the sphere boundization and the paraboloid boundization. Recall that a right circular cone is a surface generated by a straight line passing through a fixed point and making a constant angle with a fixed straight line passing through the given point. The fixed point is called the vertex of the right circular conic,the fixed straight line is called the axis of the conic and the constant angle is called the semi-vertical angle of the conic. And a right circular conic can be described when a right triangle is rotated about one of its side containing the right triangle. Let $P=\left(\gamma_{c} u, \overrightarrow{0}\right)$ be a fixed point in $\mathbb{H}_{N}$ where $\gamma_{c} \in \mathbb{C}$ is a fixed number and $Q=(\overrightarrow{0}, q)$ be an arbitrary point in $\mathbb{H}_{N}$ and let $Z=(c u, z) \in \mathbb{H}_{N}$ 


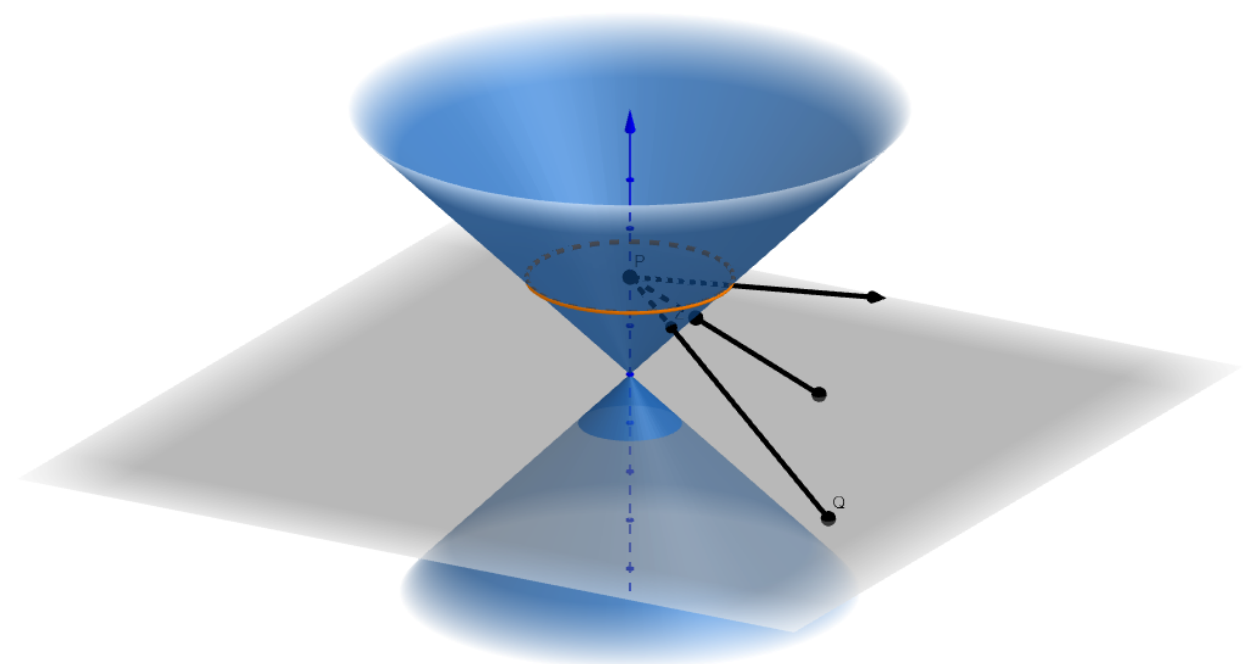

Figure 3.3: Cone

lie on a surface of a conic. If $P, Z$ and $Q$ lie on the straight line then the vectors $\overrightarrow{P Q}$ and $\overrightarrow{P Z}$ are collinear and impose two conditions the collinearity

$$
\overrightarrow{P Z}=t_{c} \overrightarrow{P Q} \text { where } t_{c} \in \mathbb{R}
$$

requires that, $\left(\left(c-\gamma_{c}\right) u, z\right)=t_{c}\left(-\gamma_{c} u, q\right)$ then

$$
z=t_{c} q \text { and } c=\left(1-t_{c}\right) \gamma_{c}
$$

Let $\hat{Z}=(\hat{c} u, \hat{z}) \in \mathbb{H}_{N}$ lies on a surface and $\hat{Q}=(\overrightarrow{0}, \hat{q})$ be an arbitrary points in $\mathbb{H}_{N}$ such that

$$
\overrightarrow{P Z}=\hat{t}_{c} \overrightarrow{P Q} \text { where } \hat{t}_{c} \in \mathbb{R}
$$

requires that, $\left(\left(\hat{c}-\gamma_{c}\right) u, \hat{z}\right)=\hat{t}_{c}\left(-\gamma_{c} u, \hat{q}\right)$ then

$$
\hat{z}=\hat{t}_{c} \hat{q} \text { and } \hat{c}=\left(1-\hat{t}_{c}\right) \gamma_{c}
$$

Now consider $Z, Q$ and $t_{c}$ as a sequences in (3.7.1) as follows $Z_{n}=\left(c_{n} u, z_{n}\right)$, $Q_{n}=\left(\overrightarrow{0}, q_{n}\right)$ and $t_{c}=t_{c n}$ so we have

$$
\overrightarrow{P Z_{n}}=t_{c n} \overrightarrow{P Q_{n}}
$$


requires that, $\left(\left(c_{n}-\gamma_{c}\right) u, z_{n}\right)=t_{c n}\left(-\gamma_{c} u, q_{n}\right)$ then

$$
z_{n}=t_{c n} q_{n} \text { and } c_{n}=\left(1-t_{c n}\right) \gamma_{c}
$$

Also consider $\hat{Z}, \hat{Q}$ and $\hat{t}_{c}$ as a sequences in (3.7.3) as follows $\hat{Z}_{n}=\left(\hat{c}_{n} u, \hat{z}_{n}\right)$, $\hat{Q}_{n}=\left(\overrightarrow{0}, \hat{q}_{n}\right)$ and $\hat{t}_{c}=\hat{t}_{c n}$ so we have

$$
{\overrightarrow{P Z_{n}}}=\hat{t}_{c n} \overrightarrow{P \hat{Q}_{n}}
$$

requires that, $\left(\left(\hat{c}_{n}-\gamma_{c}\right) u, \hat{z}_{n}\right)=\hat{t}_{c n}\left(-\gamma_{c} u, \hat{q}_{n}\right)$ then

$$
\hat{z}_{n}=\hat{t}_{c n} \hat{q}_{n} \text { and } \hat{c}_{n}=\left(1-\hat{t}_{c n}\right) \gamma_{c}
$$

To find the equation of a right circular conic with a vertex $V=\left(\theta_{c} u, \overrightarrow{0}\right)$ and the semi-vertical angle $\phi$ we assume that $Z=(c u, z)$ lies on a surface of a conic and $C=(c u, \overrightarrow{0})$ lies on the axis of the conic. Then $\overrightarrow{C Z}$ is perpendicular to $\overrightarrow{C V}$, in the right angled triangle $V C P, \tan \phi=\frac{\|\overrightarrow{C Z}\|_{H_{N}}}{\|\overrightarrow{C V}\|_{H_{N}}}$. Since $\overrightarrow{C V}$ and tna $\phi$ are constant it follows that $\overrightarrow{C Z}$ is constant for all points $Z=(c u, z)$ on the surface of a conic, thus the section is a circle. Now we develop an equation for conic as following.

$$
\begin{aligned}
\|\overrightarrow{C Z}\|_{H_{N}}=\|(\overrightarrow{0}, z)\|_{H_{N}} & =\sqrt{\langle(\overrightarrow{0}, z)\rangle_{H_{N}}} \\
& =\sqrt{\langle\overrightarrow{0}, \overrightarrow{0}\rangle+\langle z, z\rangle}=\sqrt{\langle z, z\rangle}=\sqrt{\|z\|^{2}}=\|z\|
\end{aligned}
$$

and

$$
\begin{aligned}
& \|\overrightarrow{C V}\|_{H_{N}} \tan \phi=\left\|\left(\left(c-\theta_{c}\right) u, \overrightarrow{0}\right)\right\|_{H_{N}} \tan \phi \\
& =\sqrt{\left\langle\left(\left(c-\theta_{c}\right) u, \overrightarrow{0}\right),\left(\left(c-\theta_{c}\right) u, \overrightarrow{0}\right)\right\rangle_{H_{N}}} \tan \phi \\
& =\sqrt{\left\langle\left(c-\theta_{c}\right) u,\left(c-\theta_{c}\right) u\right\rangle+\langle\overrightarrow{0}, \overrightarrow{0}\rangle} \tan \phi \\
& =\sqrt{\left(c-\theta_{c}\right)\left(c-\theta_{c}\right)}\langle u, u\rangle \tan \phi \\
& =\sqrt{\left(c-\theta_{c}\right) \overline{\left(c-\theta_{c}\right)}} \tan \phi
\end{aligned}
$$


Since $c=r \theta_{c}$ for some real positive number $r$ then we have

$$
\begin{aligned}
\sqrt{\left(c-\theta_{c}\right) \overline{\left(c-\theta_{c}\right)}} \tan \phi & =\sqrt{\left(r \theta_{c}-\theta_{c}\right) \overline{\left(r \theta_{c}-\theta_{c}\right)}} \tan \phi \\
& =\sqrt{(r-1) \theta_{c} \overline{(r-1) \theta_{c}}} \tan \phi \\
& =\sqrt{(r-1)^{2} \theta_{c} \bar{\theta}_{c}} \tan \phi=(r-1) \sqrt{\theta_{c} \bar{\theta}_{c}} \tan \phi \\
& =r \sqrt{\theta_{c} \bar{\theta}_{c}} \tan \phi-\sqrt{\theta_{c} \bar{\theta}_{c}} \tan \phi \\
& =\sqrt{r^{2} \theta_{c} \bar{\theta}_{c}} \tan \phi-\sqrt{\theta_{c} \bar{\theta}_{c}} \tan \phi \\
& =\sqrt{r \theta_{c} \bar{r}_{c}} \tan \phi-\sqrt{\theta_{c} \bar{\theta}_{c}} \tan \phi \\
& =\sqrt{c \bar{c}} \tan \phi-\sqrt{\theta_{c} \bar{\theta}_{c}} \tan \phi
\end{aligned}
$$

We use the fact that $\|\overrightarrow{C V}\|_{H_{N}} \tan \phi=\|\overrightarrow{C Z}\|_{H_{N}}$ to write the equation of the conic as follows

$$
\sqrt{c \bar{c}} \tan \phi-\sqrt{\theta_{c} \bar{\theta}_{c}} \tan \phi=\|z\|
$$

therefore

$$
\sqrt{c \bar{c}}=\|z\| \cot \phi+\sqrt{\theta_{c} \bar{\theta}_{c}}
$$

since $c=\left(1-t_{c}\right) \gamma_{c}$ then we have

$$
\sqrt{\left(1-t_{c}\right) \gamma_{c} \overline{\left(1-t_{c}\right) \gamma_{c}}}=\|z\| \cot \phi+\sqrt{\theta_{c} \bar{\theta}_{c}}
$$

use the fact that $1-t_{c}$ is a real number and simplify to get

$$
\left(1-t_{c}\right) \sqrt{\gamma_{c} \overline{\gamma_{c}}}=\|z\| \cot \phi+\sqrt{\theta_{c} \bar{\theta}_{c}}
$$

Notice that $\sqrt{\gamma_{c} \overline{\gamma_{c}}}=\left|\gamma_{c}\right|$ and $\sqrt{\theta \bar{\theta}}=\left|\theta_{c}\right|$, and assume that $\cot \phi=m$ thus we have

$$
\left(1-t_{c}\right)\left|\gamma_{c}\right|=m\|z\|+\left|\theta_{c}\right|
$$

Solve for $t_{c}$ to obtain

$$
t_{c}=1-\frac{m\|z\|+\left|\theta_{c}\right|}{\left|\gamma_{c}\right|}
$$

We want to express $t_{c}$ as a function of $q$ by (3.7.2) we obtain

$$
\|z\|^{2}=\langle z, z\rangle=\left\langle t_{c} q, t_{c} q\right\rangle=t_{c}^{2}\langle q, q\rangle=t_{c}^{2}\|q\|^{2}, \quad\|z\|=\left|t_{c}\right|\|q\|
$$


Combining (3.7.13) with $c=\left(1-t_{c}\right) \gamma_{c}$ we get

$$
t_{c}=1-\frac{t_{c} m\|q\|+\left|\theta_{c}\right|}{\sqrt{\gamma_{c} \overline{\gamma_{c}}}}
$$

Assume that $\alpha_{c}=\frac{\left|\gamma_{c}\right|-\left|\theta_{c}\right|}{m}$ and solving the equation $\left|\gamma_{c}\right| t_{c}=\left|\gamma_{c}\right|-m\|q\| t_{c}-\left|\theta_{c}\right|$ for $t_{c}$ to get

$$
t_{c}=\frac{\left|\gamma_{c}\right|-\left|\theta_{c}\right|}{m\|q\|+\left|\gamma_{c}\right|}=\frac{\frac{\left|\gamma_{c}\right|-\left|\theta_{c}\right|}{m}}{\|q\|+\frac{\left|\gamma_{c}\right|}{m}}=\frac{\alpha_{c}}{\|q\|+\frac{\left|\gamma_{c}\right|}{m}}
$$

Proposition 3.7.1 Consider $t_{c}$ subject to (3.7.16) and assume $\rho_{2}>\rho_{1}$ with $\|q\| \leq \rho_{1}$ and $\|q\| \geq \rho_{2}$ then we have

$$
\frac{\alpha_{c}}{\rho_{1}+\frac{\left|\gamma_{c}\right|}{m}} \leq t_{c} \leq \frac{m \alpha_{c}}{\left|\gamma_{c}\right|}
$$

Moreover, $t_{c} \longrightarrow 0$ as $\|q\| \longrightarrow \infty$

Proof For $\|q\| \geq \rho_{2}$ we have

$$
t_{c}=\frac{\alpha_{c}}{\|q\|+\frac{\left|\gamma_{c}\right|}{m}} \leq \frac{\alpha_{c}}{\frac{\left|\gamma_{c}\right|}{m}}=\frac{m \alpha_{c}}{\left|\gamma_{c}\right|}
$$

For $\|q\| \leq \rho_{1}$ we have

$$
t_{c}=\frac{\alpha_{c}}{\|q\|+\frac{\left|\gamma_{c}\right|}{m}} \geq \frac{\alpha_{c}}{\rho_{1}+\frac{\left|\gamma_{c}\right|}{m}}
$$

Consider $z_{n}$ subject to (3.7.6) then for $q_{n} \neq 0$ we have

$$
z_{n}=\frac{\alpha_{c} q_{n}}{\left\|q_{n}\right\|+\frac{\left|\gamma_{c}\right|}{m}}=\frac{q_{n}}{\left\|q_{n}\right\|}\left(\frac{\alpha_{c}}{1+\frac{\left|\gamma_{c}\right|}{m\left\|q_{n}\right\|}}\right)
$$

Let $\hat{Z}=(\hat{c} u, \hat{z})$ lies on a conic surface and consider $\hat{t}_{c}$ subject to (3.7.3) then for $\hat{q}_{n} \neq 0$ we have

$$
\hat{t}_{c}=\frac{\alpha_{c}}{\|\hat{q}\|+\frac{\left|\gamma_{c}\right|}{m}}
$$

and

$$
\begin{gathered}
\hat{z}_{n}=\hat{t}_{c n} \hat{q}_{n} \text { and } \hat{c}_{n}=\left(1-\hat{t}_{c n}\right) \gamma_{c} \\
\hat{z}_{n}=\frac{\hat{q_{n}}}{\| \hat{q_{n} \|}}\left(\frac{\alpha_{c}}{1+\frac{\left|\gamma_{c}\right|}{m\left\|\hat{q}_{n}\right\|}}\right)
\end{gathered}
$$


Proposition 3.7.2 Consider a sequences $t_{c n}$ and $q_{n} \neq 0$ subject to (3.7.6) then we have

$$
t_{c n} \sim \frac{\alpha_{c}}{\left\|q_{n}\right\|} \quad \text { as } n \longrightarrow \infty
$$

and the coefficient of $\frac{q_{n}}{\left\|q_{n}\right\|}=U_{n}$ in (3.7.17) tends to $\alpha_{c}$ as $n \longrightarrow \infty$

Proposition 3.7.3 Consider a sequences $\hat{t}_{c n}$ and $\hat{q}_{n} \neq 0$ subject to (3.6.8) then we have

$$
\hat{t}_{c n} \sim \frac{\alpha_{c}}{\left\|\hat{q_{n}}\right\|} \text { as } n \longrightarrow \infty
$$

and the coefficient of $\frac{\hat{q_{n}}}{\left\|\hat{q_{n}}\right\|}=\hat{U}_{n}$ in $(3.7 .20)$ tends to $\alpha_{c}$ as $n \longrightarrow \infty$.

Notice that $t_{c n} \longrightarrow 0$ and $\hat{t}_{c n} \longrightarrow 0$ when $n \longrightarrow \infty$.

\subsection{A Hyperboloid Boundization}

The forth example of a general surface boundization is a hyperboloid boundization. A hyperbola is the set of points such that the difference of the distance of each point from two fixed points, called the focus, is a constant. The midpoint the line segment joining the focus points is the center of the hyperbola. With these ideas in mind, we are now ready to find the equation of a hyperbola. First, place the center at $(\overrightarrow{0}, \overrightarrow{0})$, next, we consider $\left(\theta_{h} u, \overrightarrow{0}\right)$ and $\left(-\theta_{h} u, \overrightarrow{0}\right)$ are the focus points. Now we let the constant difference of the distances from any point $Z=(c u, z)$ on the hyperbola to $\left(\theta_{h} u, \overrightarrow{0}\right)$ and $\left(-\theta_{h} u, \overrightarrow{0}\right)$ be denoted by $\pm 2 b$. The point $Z=(c u, z)$ must satisfy the equation

$$
\left\|(c u, z)-\left(-\theta_{h} u, \overrightarrow{0}\right)\right\|_{H_{N}}-\left\|(c u, z)-\left(\theta_{h} u, \overrightarrow{0}\right)\right\|_{H_{N}}= \pm 2 b
$$

If a point $Z=(c u, z)$ is on upper branch, the positive sign is used, and if a point $Z=(c u, z)$ is on lower branch, the negative sign is used. Without loss of generality we assume the point $Z=(c u, z)$ is on upper branch then

$$
\left\|\left(c u+\theta_{h} u, z\right)\right\|_{H_{N}}-\left\|\left(c u-\theta_{h} u, z\right)\right\|_{H_{N}}=2 b
$$

or

$$
\sqrt{\left\langle\left(c u+\theta_{h} u, z\right),\left(c u+\theta_{h} u, z\right)\right\rangle_{H_{N}}}-\sqrt{\left\langle\left(c u-\theta_{h} u, z\right),\left(c u-\theta_{h} u, z\right)\right\rangle_{H_{N}}}=2 b
$$

isolate one radical 


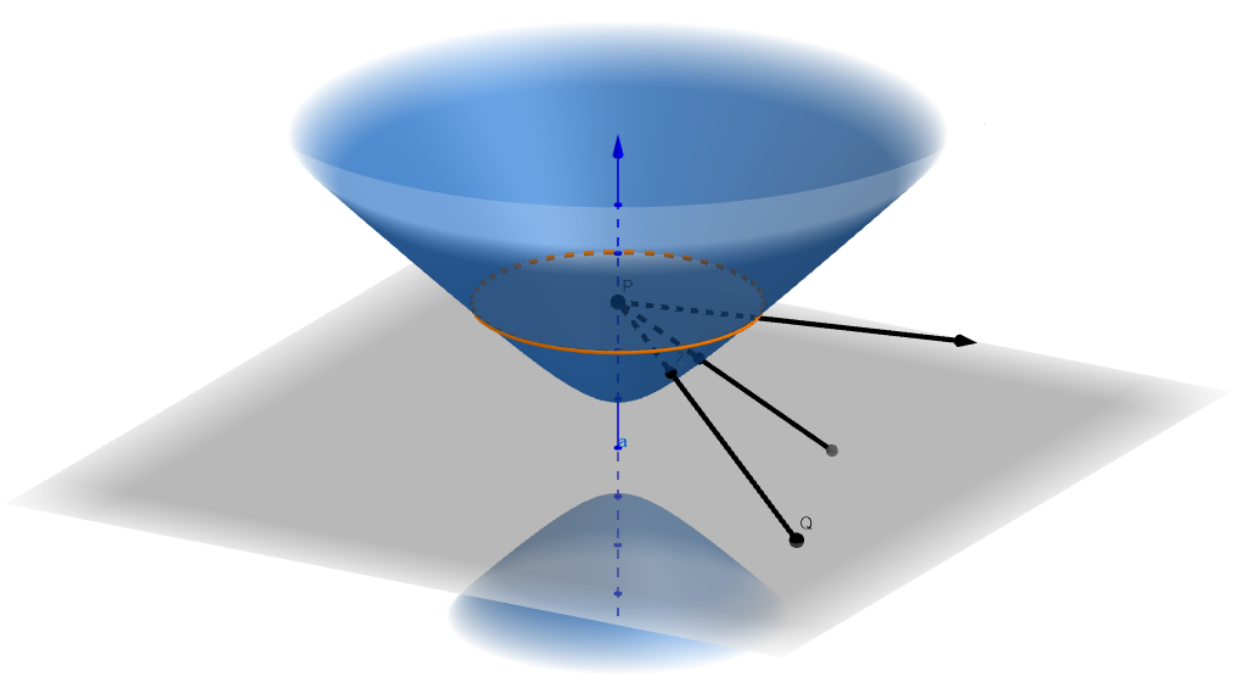

Figure 3.4: Hyparaboloid

$$
\begin{aligned}
& \sqrt{\left\langle\left(\left(c+\theta_{h}\right) u, z\right),\left(\left(c+\theta_{h}\right) u, z\right)\right\rangle_{H_{N}}} \\
& =\sqrt{\left\langle\left(\left(c-\theta_{h}\right) u, z\right),\left(\left(c-\theta_{h}\right) u, z\right)\right\rangle_{H_{N}}}+2 b
\end{aligned}
$$

square both sides

$$
\begin{aligned}
& \left\langle\left(\left(c+\theta_{h}\right) u, z\right),\left(\left(c+\theta_{h}\right) u, z\right)\right\rangle_{H_{N}}=\left\langle\left(\left(c-\theta_{h}\right) u, z\right),\left(\left(c-\theta_{h}\right) u, z\right)\right\rangle_{H_{N}} \\
& +4 b \sqrt{\left\langle\left(\left(c-\theta_{h}\right) u, z\right),\left(\left(c-\theta_{h}\right) u, z\right)\right\rangle_{H_{N}}}+4 b^{2}
\end{aligned}
$$

Next we use the definition of the new inner product to get.

$$
\begin{aligned}
& \left\langle\left(c+\theta_{h}\right) u,\left(c+\theta_{h}\right) u\right\rangle+\langle z, z\rangle-\left\langle\left(c-\theta_{h}\right) u,\left(c-\theta_{h}\right) u\right\rangle-\langle z, z\rangle \\
& =4 b \sqrt{\left\langle\left(c-\theta_{h}\right) u,\left(c-\theta_{h}\right) u\right\rangle+\langle z, z\rangle}+4 b^{2}
\end{aligned}
$$

or

$$
\left(c+\theta_{h}\right) \overline{\left(c+\theta_{h}\right)}-\left(c-\theta_{h}\right) \overline{\left(c-\theta_{h}\right)}=4 b \sqrt{\left(c-\theta_{h}\right) \overline{\left(c-\theta_{h}\right)}+\langle z, z\rangle}+4 b^{2}
$$

we remove the parentheses.

$$
\begin{aligned}
& c \bar{c}+c \bar{\theta}_{h}+\theta_{h} \bar{c}+\theta_{h} \bar{\theta}_{h}-\left(c \bar{c}-c \bar{\theta}_{h}-\theta_{h} \bar{c}+\theta_{h} \bar{\theta}_{h}\right) \\
& =4 b \sqrt{c \bar{c}-c \bar{\theta}_{h}-\theta_{h} \bar{c}+\theta_{h} \bar{\theta}_{h}+\langle z, z\rangle}+4 b^{2}
\end{aligned}
$$


simplify and isolate the radical

$$
2 c \bar{\theta}_{h}+2 \theta_{h} \bar{c}-4 b^{2}=4 b \sqrt{c \bar{c}-2 \operatorname{Re}\left(\theta_{h} \bar{c}\right)+\theta_{h} \bar{\theta}_{h}+\langle z, z\rangle}
$$

Since $2 c \bar{\theta}_{h}+2 \theta_{h} \bar{c}=2\left(\overline{\bar{c}}_{h}+\theta_{h} \bar{c}\right)=4 \operatorname{Re}\left(\theta_{h} \bar{c}\right)$ then

$$
4 \operatorname{Re}\left(\theta_{h} \bar{c}\right)-4 b^{2}=4 b \sqrt{c \bar{c}-2 \operatorname{Re}\left(\theta_{h} \bar{c}\right)+\theta_{h} \bar{\theta}_{h}+\langle z, z\rangle}
$$

divide each side by 4

$$
\operatorname{Re}\left(\theta_{h} \bar{c}\right)-b^{2}=b \sqrt{c \bar{c}-2 \operatorname{Re}\left(\theta_{h} \bar{c}\right)+\theta_{h} \bar{\theta}_{h}+\langle z, z\rangle}
$$

square both sides

$$
\left(\operatorname{Re}\left(\theta_{h} \bar{c}\right)-b^{2}\right)^{2}=b^{2}\left(c \bar{c}-2 \operatorname{Re}\left(\theta_{h} \bar{c}\right)+\theta_{h} \bar{\theta}_{h}+\langle z, z\rangle\right)
$$

simplify

$$
\left(\operatorname{Re}\left(\theta_{h} \bar{c}\right)\right)^{2}-2 b^{2} \operatorname{Re}\left(\theta_{h} \bar{c}\right)+b^{4}=b^{2}\left(c \bar{c}-2 \operatorname{Re}\left(\theta_{h} \bar{c}\right)+\theta_{h} \bar{\theta}_{h}+\|z\|^{2}\right)
$$

thus we have

$$
\left(\operatorname{Re}\left(\theta_{h} \bar{c}\right)\right)^{2}+b^{4}=b^{2} c \bar{c}+b^{2} \theta_{h} \bar{\theta}_{h}+b^{2}\|z\|^{2}
$$

therefore the equation of a hyperbola is

$$
\left(\operatorname{Re}\left(\theta_{h} \bar{c}\right)\right)^{2}-b^{2} c \bar{c}-b^{2}\|z\|^{2}=b^{2} \theta_{h} \bar{\theta}_{h}-b^{4}
$$

Let $P=\left(\gamma_{h} u, \overrightarrow{0}\right)$ be a fixed point in $\mathbb{H}_{N}$ where $\gamma_{h} \in \mathbb{C}$ is a fixed number and $Q=(\overrightarrow{0}, q)$ be an arbitrary point in $\mathbb{H}_{N}$ and let $Z=(c u, z) \in \mathbb{H}_{N}$ lie on a surface of a hyperbola. If $P, Z$ and $Q$ lie on the straight line then the vectors $\overrightarrow{P Q}$ and $\overrightarrow{P Z}$ are collinear and impose two conditions the collinearity

$$
\overrightarrow{P Z}=t_{h} \overrightarrow{P Q} \text { where } t_{h} \in \mathbb{R}
$$

requires that, $\left(\left(c-\gamma_{h}\right) u, z\right)=t_{h}\left(-\gamma_{h} u, q\right)$ then

$$
z=t_{h} q \text { and } c=\left(1-t_{h}\right) \gamma_{h}
$$

Let $\hat{Z}=(\hat{c} u, \hat{z}) \in \mathbb{H}_{N}$ lies on a surface and $\hat{Q}=(\overrightarrow{0}, \hat{q})$ be an arbitrary points in $\mathbb{H}_{N}$ such that

$$
\overrightarrow{P Z}=\hat{t}_{h} \overrightarrow{P Q} \quad \text { where } \hat{t}_{h} \in \mathbb{R}
$$


requires that, $\left(\left(\hat{c}-\gamma_{h}\right) u, \hat{z}\right)=\hat{t}_{h}\left(-\gamma_{h} u, \hat{q}\right)$ then

$$
\hat{z}=\hat{t}_{h} \hat{q} \text { and } \hat{c}=\left(1-\hat{t}_{h}\right) \gamma_{h}
$$

Now consider $Z, Q$ and $t_{h}$ as a sequences in (3.8.2) as follows $Z_{n}=\left(c_{n} u, z_{n}\right)$, $Q_{n}=\left(\overrightarrow{0}, q_{n}\right)$ and $t_{h}=t_{h n}$ so we have

$$
\overrightarrow{P Z_{n}}=t_{h n} \overrightarrow{P Q_{n}}
$$

requires that, $\left(\left(c_{n}-\gamma_{h}\right) u, z_{n}\right)=t_{h n}\left(-\gamma_{h} u, q_{n}\right)$ then

$$
z_{n}=t_{h n} q_{n} \text { and } c_{n}=\left(1-t_{h n}\right) \gamma_{h}
$$

Also consider $\hat{Z}, \hat{Q}$ and $\hat{t}_{h}$ as a sequences in (3.8.4) as follows $\hat{Z}_{n}=\left(\hat{c}_{n} u, \hat{z}_{n}\right)$, $\hat{Q}_{n}=\left(\overrightarrow{0}, \hat{q}_{n}\right)$ and $\hat{t}_{h}=\hat{t}_{h n}$ so we have

$$
\vec{P}_{n}=\hat{t}_{h n} \vec{P}_{n}
$$

requires that, $\left(\left(\hat{c}_{n}-\gamma_{h}\right) u, \hat{z}_{n}\right)=\hat{t}_{h n}\left(-\gamma_{h} u, \hat{q}_{n}\right)$ then

$$
\hat{z}_{n}=\hat{t}_{h n} \hat{q}_{n} \text { and } \hat{c}_{n}=\left(1-\hat{t}_{h n}\right) \gamma_{h}
$$

Substitute $c=\left(1-t_{h}\right) \gamma_{h}$ in (3.8.1) we get

$$
\left(R e\left(\theta_{h} \overline{\left(1-t_{h}\right) \gamma_{h}}\right)\right)^{2}-b^{2}\left(\left(1-t_{h}\right) \gamma_{h}\right) \overline{\left(1-t_{h}\right) \gamma_{h}}-b^{2}\|z\|^{2}=b^{2} \theta_{h} \bar{\theta}_{h}-b^{4}
$$

use the fact that $1-t_{h}$ is a real number and simplify to get

$$
\left(1-t_{h}\right)^{2}\left(\operatorname{Re}\left(\theta_{h} \bar{\gamma}_{h}\right)\right)^{2}-b^{2}\left(1-t_{h}\right)^{2} \gamma_{h} \bar{\gamma}_{h}-b^{2}\|z\|^{2}=b^{2} \theta_{h} \bar{\theta}_{h}-b^{4}
$$

rearrange terms

$$
\left(1-t_{h}\right)^{2}\left(\left(\operatorname{Re}\left(\theta_{h} \bar{\gamma}_{h}\right)\right)^{2}-b^{2} \gamma_{h} \bar{\gamma}_{h}\right)-b^{2}\|z\|^{2}=b^{2} \theta_{h} \bar{\theta}_{h}-b^{4}
$$

or

$$
\left(1-t_{h}\right)^{2}\left(\gamma_{h} \bar{\gamma}_{h}-\left(\frac{\operatorname{Re}\left(\theta_{h} \bar{\gamma}_{h}\right)}{b}\right)^{2}\right)+\|z\|^{2}+\theta_{h} \bar{\theta}_{h}-b^{2}=0
$$

Let $\mathcal{C}=\left(\frac{\operatorname{Re}\left(\theta_{h} \bar{\gamma}_{h}\right)}{b}\right)^{2}-\gamma_{h} \bar{\gamma}_{h}$ and $\alpha_{h}=-\mathcal{C}+\theta_{h} \bar{\theta}_{h}-b^{2}$. We make two assumptions 
1. $\mathcal{C}>0$

2. $\alpha_{h}<0$

We need the first assumption in order to satisfy the triangle inequality as following

$$
\left\|\left(c u+\theta_{h} u, z\right)\right\|_{H_{N}}=\left\|\left(c u-\theta_{h} u, z\right)\right\|_{H_{N}}+2 b
$$

and use a triangle with a vertices $(c u, z),\left(\theta_{h} u, \overrightarrow{0}\right)$ and $\left(-\theta_{h} u, \overrightarrow{0}\right)$ so the distances between $\left(\theta_{h}, \overrightarrow{0}\right)$ and $\left(-\theta_{h}, \overrightarrow{0}\right)$ is

$$
\begin{aligned}
\left\|\left(\theta_{h}, \overrightarrow{0}\right)-\left(-\theta_{h}, \overrightarrow{0}\right)\right\|_{H_{N}} & =\left\|\left(2 \theta_{h} u, \overrightarrow{0}\right)\right\|_{H_{N}}=\sqrt{\left\langle 2 \theta_{h} u, 2 \theta_{h} u\right\rangle} \\
& =\sqrt{2 \theta_{h} \overline{2 \theta}_{h}\langle u, u\rangle}=\sqrt{4 \theta_{h} \bar{\theta}_{h}}=2 \sqrt{\theta_{h} \overline{\theta_{h}}}=2\left|\theta_{h}\right|
\end{aligned}
$$

Use the fact that the sum of the lengths of any two sides of a triangle is greater than the length of the third side, so from (3.8.11) we have

$$
2\left|\theta_{h}\right|+\left\|\left(c u-\theta_{h} u, z\right)\right\|_{H_{N}}>\left\|\left(c u+\theta_{h} u, z\right)\right\|_{H_{N}}
$$

by (3.8.10) we have

$$
2\left|\theta_{h}\right|+\left\|\left(c u-\theta_{h} u, z\right)\right\|_{H_{N}}>\left\|\left(c u-\theta_{h} u, z\right)\right\|_{H_{N}}+2 b
$$

thus we have $2|\theta|>2 b$ divide each side by 2 we get $\left|\theta_{h}\right|>b$. We know that any complex number can be considered as polar form. In fact let $\gamma_{h}=\rho_{1} e^{i \mu}$ and $\theta_{h}=\rho_{2} e^{i \nu}$ Since $\gamma_{h}$ and $\theta_{h}$ in the same direction so $\mu=\nu$ therefore

$$
\mathcal{C}=\left(\frac{\operatorname{Re}\left(\theta_{h} \bar{\gamma}_{h}\right)}{b}\right)^{2}-\gamma_{h} \bar{\gamma}_{h}=\left(\frac{\rho_{1} \rho_{2} \cos (\mu-\nu)}{b}\right)^{2}-\rho_{1}^{2}=\frac{\rho_{1}^{2} \rho_{2}^{2}}{b^{2}}-\rho_{1}^{2}
$$

since $\left|\theta_{h}\right|>b$ then $\rho_{1}^{2}>b^{2}$ therefore

$$
\mathcal{C}=\frac{\rho_{1}^{2} \rho_{2}^{2}}{b^{2}}-\rho_{1}^{2}=\rho_{1}^{2}\left(\frac{\rho_{1}^{2}}{b^{2}}-1\right)>0
$$

We must assume second assumption in order to avoid complex values for $t_{h}$ for large $\|q\|$

$$
-\mathcal{C} t_{h}^{2}+2 \mathcal{C} t_{h}+\|z\|^{2}+\alpha_{h}=0
$$


solve for $t_{h}$ to obtain

$$
t_{h}=\frac{-2 \mathcal{C} \pm \sqrt{4 \mathcal{C}^{2}+4 \mathcal{C}\left(\|z\|^{2}+\alpha_{h}\right)}}{-2 \mathcal{C}}
$$

we always choose

$$
t_{h}=1-\frac{\sqrt{\mathcal{C}^{2}+\mathcal{C}\left(\|z\|^{2}+\alpha_{h}\right)}}{\mathcal{C}}
$$

We want to express $t_{h}$ as a function of $q$, from (3.8.12) we obtain

$$
\left(\|q\|^{2}-\mathcal{C}\right) t_{h}^{2}+2 \mathcal{C} t_{h}+\alpha_{h}=0
$$

Proposition 3.8.1 If $\|q\|^{2}=\mathcal{C}$ then the solution of (3.8.14) for $t_{h}$ is

$$
t_{h}=\frac{-\alpha_{h}}{2 \mathcal{C}}>0
$$

Moreover

$$
0<t_{h}<\frac{1}{2}
$$

Proof When $\|q\|^{2}=\mathcal{C}$ the equation (3.8.14) become $2 \mathcal{C} t_{h}+\alpha_{h}=0$, so the solution for $t_{h}$ is

$$
t_{h}=\frac{-\alpha_{h}}{2 \mathcal{C}}=\frac{\mathcal{C}-\theta_{h} \bar{\theta}_{h}+b^{2}}{2 \mathcal{C}}=\frac{1}{2}+\frac{-\theta_{h} \bar{\theta}_{h}+b^{2}}{2 \mathcal{C}}
$$

From our assumption $\alpha_{h}<0$ so $-\mathcal{C}+\theta_{h} \bar{\theta}_{h}-b^{2}<0$ or $0>-\theta_{h} \bar{\theta}_{h}+b^{2}>-\mathcal{C}$ this is implies that $\frac{-1}{2}<\frac{-\theta_{h} \bar{\theta}_{h}+b^{2}}{2 \mathcal{C}}<0$ since

then $t_{h}$ in this case satisfy the following inequality

$$
0<t_{h}<\frac{1}{2}
$$

Proposition 3.8.2 If $\|q\|^{2}-\mathcal{C}>0$ then the solution of (3.8.14) for $t_{h}$ is

$$
\begin{aligned}
0<t_{h} & =\frac{-\mathcal{C}+\sqrt{\mathcal{C}^{2}-\left(\|q\|^{2}-\mathcal{C}\right) \alpha_{h}}}{\left(\|q\|^{2}-\mathcal{C}\right)} \\
& =\frac{-\alpha_{h}}{\mathcal{C}+\sqrt{\mathcal{C}^{2}-\left(\|q\|^{2}-\mathcal{C}\right) \alpha_{h}}}<1
\end{aligned}
$$

and

$$
t_{h}=\frac{-\mathcal{C}-\sqrt{\mathcal{C}^{2}-\left(\|q\|^{2}-\mathcal{C}\right) \alpha_{h}}}{\left(\|q\|^{2}-\mathcal{C}\right)}<0
$$


Proof From our assumption $\|q\|^{2}-\mathcal{C}>0$ and by the fact $\mathcal{C}>0$ then easy to show that

$$
t_{h}=\frac{-\mathcal{C}+\sqrt{\mathcal{C}^{2}-\left(\|q\|^{2}-\mathcal{C}\right) \alpha_{h}}}{\left(\|q\|^{2}-\mathcal{C}\right)}>0
$$

Since $\alpha_{h} \neq 0$ so we have

$$
\begin{aligned}
\lim _{\|q\|^{2} \longrightarrow \mathcal{C}} t_{h} & =\lim _{\|q\|^{2} \longrightarrow \mathcal{C}} \frac{-\mathcal{C}+\sqrt{\mathcal{C}^{2}-\left(\|q\|^{2}-\mathcal{C}\right) \alpha_{h}}}{\left(\|q\|^{2}-\mathcal{C}\right)} \\
& =\lim _{\|q\|^{2} \longrightarrow \mathcal{C}} \frac{-\mathcal{C}+\sqrt{\mathcal{C}^{2}-\left(\|q\|^{2}-\mathcal{C}\right) \alpha_{h}}}{\left(\|q\|^{2}-\mathcal{C}\right)}\left(\frac{\mathcal{C}+\sqrt{\mathcal{C}^{2}-\left(\|q\|^{2}-\mathcal{C}\right) \alpha_{h}}}{\mathcal{C}+\sqrt{\mathcal{C}^{2}-\left(\|q\|^{2}-\mathcal{C}\right) \alpha_{h}}}\right) \\
& =\lim _{\|q\|^{2} \longrightarrow \mathcal{C}} \frac{\mathcal{C}^{2}-\left(\|q\|^{2}-\mathcal{C}\right) \alpha_{h}-\mathcal{C}^{2}}{\left(\|q\|^{2}-\mathcal{C}\right)\left(\mathcal{C}+\sqrt{\mathcal{C}^{2}-\left(\|q\|^{2}-\mathcal{C}\right) \alpha_{h}}\right)} \\
& =\lim _{\|q\|^{2} \longrightarrow \mathcal{C}} \frac{-\alpha_{h}}{\mathcal{C}+\sqrt{\mathcal{C}^{2}-\left(\|q\|^{2}-\mathcal{C}\right) \alpha_{h}}}=\frac{-\alpha_{h}}{2 \mathbb{C}}
\end{aligned}
$$

since $-\alpha_{h}=\mathcal{C}-\theta_{h} \bar{\theta}_{h}+b^{2}$ so $-\alpha_{h}<\mathcal{C}$ thus $-\alpha_{h}<\mathcal{C}+\sqrt{\mathcal{C}^{2}-\left(\|q\|^{2}-\mathcal{C}\right) \alpha_{h}}$ therefore Since $\|q\|^{2}-\mathcal{C}>0$ then we have

$$
t_{h}=\frac{-\alpha_{h}}{\mathcal{C}+\sqrt{\mathcal{C}^{2}-\left(\|q\|^{2}-\mathcal{C}\right) \alpha_{h}}}<1
$$

Since

$$
-\mathcal{C}-\sqrt{\mathcal{C}^{2}-\left(\|q\|^{2}-\mathcal{C}\right) \alpha_{h}}<0
$$

therefore

$$
t_{h}=\frac{-\mathcal{C}-\sqrt{\mathcal{C}^{2}-\left(\|q\|^{2}-\mathcal{C}\right) \alpha_{h}}}{\left(\|q\|^{2}-\mathcal{C}\right)}<0
$$

Proposition 3.8.3 If $\|q\|^{2}-\mathcal{C}<0$ then the solution of (3.8.14) for $t_{h}$ is

$$
t_{h}=\frac{-\mathcal{C}+\sqrt{\mathcal{C}^{2}-\left(\|q\|^{2}-\mathcal{C}\right) \alpha_{h}}}{\left(\|q\|^{2}-\mathcal{C}\right)}<0
$$

and

$$
t_{h}=\frac{-\mathcal{C}-\sqrt{\mathcal{C}^{2}-\left(\|q\|^{2}-\mathcal{C}\right) \alpha_{h}}}{\left(\|q\|^{2}-\mathcal{C}\right)}>0
$$


Proof From our assumption $\|q\|^{2}-\mathcal{C}<0$ and by the fact $\mathcal{C}>0$ then easy to show that

$$
t_{h}=\frac{-\mathcal{C}+\sqrt{\mathcal{C}^{2}-\left(\|q\|^{2}-\mathcal{C}\right) \alpha_{h}}}{\left(\|q\|^{2}-\mathcal{C}\right)}<0
$$

Since

$$
-\mathcal{C}-\sqrt{\mathcal{C}^{2}-\left(\|q\|^{2}-\mathcal{C}\right) \alpha_{h}}<0
$$

therefore

$$
t_{h}=\frac{-\mathcal{C}-\sqrt{\mathcal{C}^{2}-\left(\|q\|^{2}-\mathcal{C}\right) \alpha_{h}}}{\left(\|q\|^{2}-\mathcal{C}\right)}>0
$$

Since $\alpha_{h} \neq 0$ so we have

$$
\begin{aligned}
& \lim _{\|q\|^{2} \longrightarrow \mathcal{C}} t_{h} \\
= & \lim _{\|q\|^{2} \longrightarrow \mathcal{C}} \frac{-\mathcal{C}-\sqrt{\mathcal{C}^{2}-\left(\|q\|^{2}-\mathcal{C}\right) \alpha_{h}}}{\left(\|q\|^{2}-\mathcal{C}\right)} \\
= & \lim _{\|q\|^{2} \longrightarrow \mathcal{C}} \frac{-\left(\mathcal{C}+\sqrt{\mathcal{C}^{2}-\left(\|q\|^{2}-\mathcal{C}\right) \alpha_{h}}\right)}{\left(\|q\|^{2}-\mathcal{C}\right)}\left(\frac{\mathcal{C}-\sqrt{\mathcal{C}^{2}-\left(\|q\|^{2}-\mathcal{C}\right) \alpha_{h}}}{\mathcal{C}-\sqrt{\mathcal{C}^{2}-\left(\|q\|^{2}-\mathcal{C}\right) \alpha_{h}}}\right) \\
= & \lim _{\|q\|^{2} \longrightarrow \mathcal{C}} \frac{-\left(\mathcal{C}^{2}-\left(\mathcal{C}^{2}-\left(\|q\|^{2}-\mathcal{C}\right) \alpha_{h}\right)\right)}{\left(\|q\|^{2}-\mathcal{C}\right)\left(\mathcal{C}+\sqrt{\mathcal{C}^{2}-\left(\|q\|^{2}-\mathcal{C}\right) \alpha_{h}}\right)} \\
= & \lim _{\|q\|^{2} \longrightarrow \mathcal{C}} \frac{\alpha_{h}}{\mathcal{C}-\sqrt{\mathcal{C}^{2}-\left(\|q\|^{2}-\mathcal{C}\right) \alpha_{h}}} \text { does not exist }
\end{aligned}
$$

We looking for $0<t_{h}<1$, and $t_{h}$ as a continuous function of $\|q\|$ we define

$$
t_{h}=\frac{-\alpha_{h}}{\mathcal{C}+\sqrt{\mathcal{C}^{2}-\left(\|q\|^{2}-\mathcal{C}\right) \alpha_{h}}}
$$

we can write $t_{h}$ as

$$
t_{h}=\frac{-\alpha_{h}}{\mathcal{C}+\sqrt{E}}
$$

where

$$
E=\mathcal{C}^{2}-\left(\|q\|^{2}-\mathcal{C}\right) \alpha_{h}
$$

which is easily shown to be $C^{\infty}$ function on all the variables $\mathcal{C}, \alpha_{h}$ and $\|q\|$ Similar we define

$$
\hat{t}_{h}=\frac{-\alpha_{h}}{\mathcal{C}+\sqrt{\hat{E}}}
$$


where

$$
\hat{E}=\mathcal{C}^{2}-\left(\|\hat{q}\|^{2}-\mathcal{C}\right) \alpha_{h}
$$

Consider $z_{n}$ subject to (3.8.7) then for $q_{n} \neq 0$ we have

$$
\begin{aligned}
z_{n} & =\frac{-q_{n} \alpha_{h}}{\mathcal{C}+\sqrt{\mathcal{C}^{2}-\left(\left\|q_{n}\right\|^{2}-\mathcal{C}\right) \alpha_{h}}} \\
& =\frac{q_{n}}{\left\|q_{n}\right\|}\left(\frac{-\alpha_{h}}{\frac{\mathcal{C}}{\left\|q_{n}\right\|}+\sqrt{\frac{\mathcal{C}^{2}}{\left\|q_{n}\right\|^{2}}-\left(1-\frac{\mathcal{C}}{\|q\|^{2}}\right) \alpha_{h}}}\right)
\end{aligned}
$$

since

$$
\hat{z}_{n}=\hat{t}_{h n} \hat{q}_{n} \text { and } \hat{c}_{n}=\left(1-\hat{t}_{h n}\right) \gamma_{h}
$$

then

$$
\frac{q_{n}}{\left\|q_{n}\right\|}\left(\frac{-\alpha_{h}}{\frac{\mathcal{C}}{\left\|q_{n}\right\|}+\sqrt{\frac{\mathcal{C}^{2}}{\left\|q_{n}\right\|^{2}}-\left(1-\frac{\mathcal{C}}{\|q\|^{2}}\right) \alpha_{h}}}\right)
$$

Proposition 3.8.4 Consider a sequences $t_{h n}$ and $q_{n} \neq 0$ subject to (3.8.7) then we have

$$
t_{h n} \sim \frac{\sqrt{-\alpha_{h}}}{\left\|q_{n}\right\|} \text { as } n \longrightarrow \infty
$$

and $t_{h n} q \sim \sqrt{-\alpha_{h}}$ as $n \longrightarrow \infty$

Proposition 3.8.5 Consider a sequences $\hat{t}_{h n}$ and $\hat{q}_{n} \neq 0$ subject to (3.8.24) then we have

$$
t_{h n} \sim \frac{\sqrt{-\alpha_{h}}}{\left\|q_{n}\right\|} \text { as } n \longrightarrow \infty
$$

and $\hat{t}_{h n} \hat{q} \sim \sqrt{-\alpha_{h}}$ as $n \longrightarrow \infty$ as $n \longrightarrow \infty$.

Notice that $t_{h n} \longrightarrow 0$ and $\hat{t}_{h n} \longrightarrow 0$ when $n \longrightarrow \infty$. 


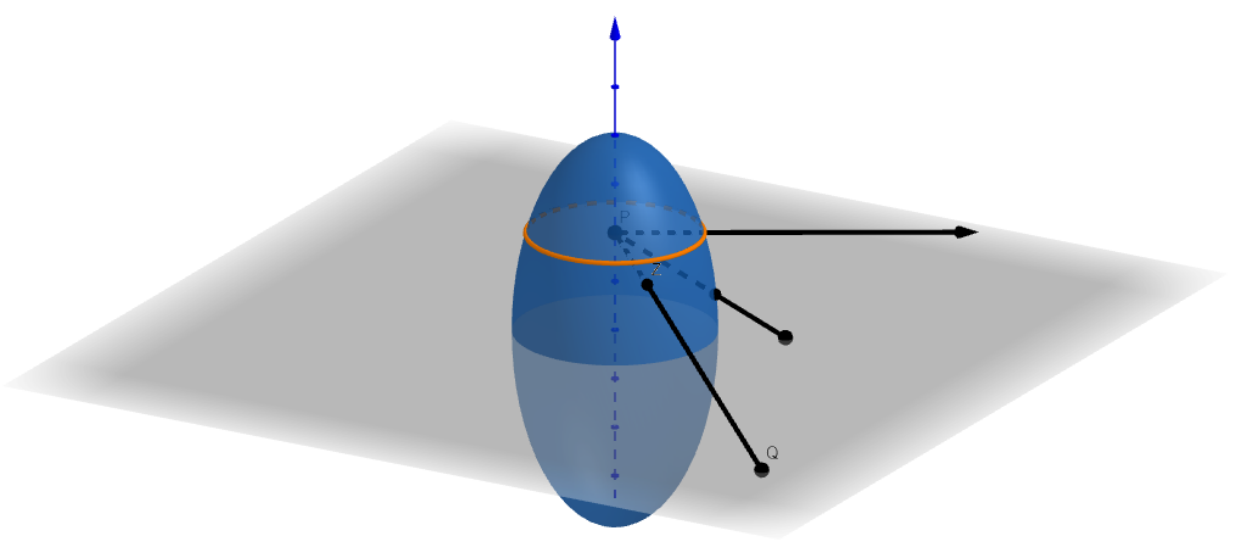

Figure 3.5: Ellipsoid

\subsection{An Ellipsoid Boundization}

The last example of a general surface boundization is the ellipsoid boundization. And as we know that the sphere is a special case of ellipsoid if the axes have the same length, so it is interesting to note that they have the similar boundization properties. An ellipse is the collection of points such that the sum of whose distance from two fixed points, called the focus, is a constant. The midpoint the line segment joining the focus points is the center of the ellipse. With these ideas in mind, we are now ready to find the equation of an ellipse. First, place the center at $(\overrightarrow{0}, \overrightarrow{0})$, next, we consider $\left(\theta_{e} u, \overrightarrow{0}\right)$ and $\left(-\theta_{e} u, \overrightarrow{0}\right)$ are the focus points. Now we let the constant sum of the distances from any point $Z=(c u, z)$ on the ellipse to $\left(\theta_{h} u, \overrightarrow{0}\right)$ and $\left(-\theta_{e} u, \overrightarrow{0}\right)$ be denoted by $2 d$. The point $Z=(c u, z)$ must satisfy the equation

$$
\left\|(c u, z)-\left(-\theta_{e} u, \overrightarrow{0}\right)\right\|_{H_{N}}+\left\|(c u, z)-\left(\theta_{e} u, \overrightarrow{0}\right)\right\|_{H_{N}}=2 d
$$

or

$$
\sqrt{\left\langle\left(c u+\theta_{e} u, z\right),\left(c u+\theta_{e} u, z\right)\right\rangle_{H_{N}}}+\sqrt{\left\langle\left(c u-\theta_{e} u, z\right),\left(c u-\theta_{e} u, z\right)\right\rangle_{H_{N}}}=2 d
$$


isolate one radical

$$
\begin{aligned}
& \sqrt{\left\langle\left(\left(c+\theta_{e}\right) u, z\right),\left(\left(c+\theta_{e}\right) u, z\right)\right\rangle_{H_{N}}} \\
& =-\sqrt{\left\langle\left(\left(c-\theta_{e}\right) u, z\right),\left(\left(c-\theta_{e}\right) u, z\right)\right\rangle_{H_{N}}}+2 d
\end{aligned}
$$

square both sides

$$
\begin{aligned}
& \left\langle\left(\left(c+\theta_{e}\right) u, z\right),\left(\left(c+\theta_{e}\right) u, z\right)\right\rangle_{H_{N}}=\left\langle\left(\left(c-\theta_{e}\right) u, z\right),\left(\left(c-\theta_{e}\right) u, z\right)\right\rangle_{H_{N}} \\
& -4 d \sqrt{\left\langle\left(\left(c-\theta_{e}\right) u, z\right),\left(\left(c-\theta_{e}\right) u, z\right)\right\rangle_{H_{N}}}+4 d^{2}
\end{aligned}
$$

Next we use the definition of the new inner product to get.

$$
\begin{aligned}
& \left\langle\left(c+\theta_{e}\right) u,\left(c+\theta_{e}\right) u\right\rangle+\langle z, z\rangle-\left\langle\left(c-\theta_{e}\right) u,\left(c-\theta_{e}\right) u\right\rangle-\langle z, z\rangle \\
& =-4 d \sqrt{\left\langle\left(c-\theta_{e}\right) u,\left(c-\theta_{e}\right) u\right\rangle+\langle z, z\rangle}+4 d^{2}
\end{aligned}
$$

or

$$
\left(c+\theta_{e}\right) \overline{\left(c+\theta_{e}\right)}-\left(c-\theta_{e}\right) \overline{\left(c-\theta_{e}\right)}=-4 d \sqrt{\left(c-\theta_{e}\right) \overline{\left(c-\theta_{e}\right)}+\langle z, z\rangle}+4 d^{2}
$$

we remove the parentheses.

$$
\begin{gathered}
c \bar{c}+c \bar{\theta}_{e}+\theta_{e} \bar{c}+\theta_{e} \bar{\theta}_{e}-\left(c \bar{c}-c \bar{\theta}_{h}-\theta_{h} \bar{c}+\theta_{h} \bar{\theta}_{e}\right) \\
=-4 d \sqrt{c \bar{c}-c \bar{\theta}_{e}-\theta_{e} \bar{c}+\theta_{e} \bar{\theta}_{e}+\langle z, z\rangle}+4 b^{2}
\end{gathered}
$$

simplify and isolate the radical

$$
2 c \bar{\theta}_{e}+2 \theta_{e} \bar{c}-4 d^{2}=-4 d \sqrt{c \bar{c}-2 R e\left(\theta_{h} \bar{c}\right)+\theta_{h} \bar{\theta}_{h}+\langle z, z\rangle}
$$

Since $2 c \bar{\theta}_{e}+2 \theta_{e} \bar{c}=2\left(\overline{\bar{c}}_{e}+\theta_{e} \bar{c}\right)=4 R e\left(\theta_{e} \bar{c}\right)$ then

$$
4 R e\left(\theta_{e} \bar{c}\right)-4 d^{2}=-4 d \sqrt{c \bar{c}-2 \operatorname{Re}\left(\theta_{e} \bar{c}\right)+\theta_{e} \bar{\theta}_{e}+\langle z, z\rangle}
$$

divide each side by 4

$$
R e\left(\theta_{e} \bar{c}\right)-d^{2}=-d \sqrt{c \bar{c}-2 R e\left(\theta_{e} \bar{c}\right)+\theta_{e} \bar{\theta}_{e}+\langle z, z\rangle}
$$

square both sides

$$
\left(\operatorname{Re}\left(\theta_{e} \bar{c}\right)-d^{2}\right)^{2}=d^{2}\left(c \bar{c}-2 \operatorname{Re}\left(\theta_{e} \bar{c}\right)+\theta_{e} \bar{\theta}_{e}+\langle z, z\rangle\right)
$$


simplify

$$
\left(\operatorname{Re}\left(\theta_{e} \bar{c}\right)\right)^{2}-2 d^{2} \operatorname{Re}\left(\theta_{e} \bar{c}\right)+d^{4}=d^{2}\left(c \bar{c}-2 \operatorname{Re}\left(\theta_{e} \bar{c}\right)+\theta_{e} \bar{\theta}_{e}+\|z\|^{2}\right)
$$

thus we have

$$
\left(\operatorname{Re}\left(\theta_{e} \bar{c}\right)\right)^{2}+d^{4}=d^{2} c \bar{c}+d^{2} \theta_{e} \bar{\theta}_{e}+d^{2}\|z\|^{2}
$$

therefore the equation of an ellipse is

$$
\left(\operatorname{Re}\left(\theta_{e} \bar{c}\right)\right)^{2}-d^{2} c \bar{c}-d^{2}\|z\|^{2}=d^{2} \theta_{e} \bar{\theta}_{e}-d^{4}
$$

Let $P=\left(\gamma_{e} u, \overrightarrow{0}\right)$ be a fixed point in $\mathbb{H}_{N}$ where $\gamma_{e} \in \mathbb{C}$ is a fixed number and $Q=(\overrightarrow{0}, q)$ be an arbitrary point in $\mathbb{H}_{N}$ and let $Z=(c u, z) \in \mathbb{H}_{N}$ lie on a surface of an ellipse. If $P, Z$ and $Q$ lie on the straight line then the vectors $\overrightarrow{P Q}$ and $\overrightarrow{P Z}$ are collinear and impose two conditions the collinearity

$$
\overrightarrow{P Z}=t_{e} \overrightarrow{P Q} \text { where } t_{e} \in \mathbb{R}
$$

requires that, $\left(\left(c-\gamma_{e}\right) u, z\right)=t_{e}\left(-\gamma_{e} u, q\right)$ then

$$
z=t_{e} q \text { and } c=\left(1-t_{e}\right) \gamma_{e}
$$

Let $\hat{Z}=(\hat{c} u, \hat{z}) \in \mathbb{H}_{N}$ lies on a surface and $\hat{Q}=(\overrightarrow{0}, \hat{q})$ be an arbitrary points in $\mathbb{H}_{N}$ such that

$$
\overrightarrow{P Z}=\hat{t}_{e} \overrightarrow{P Q} \quad \text { where } \hat{t}_{e} \in \mathbb{R}
$$

requires that, $\left(\left(\hat{c}-\gamma_{e}\right) u, \hat{z}\right)=\hat{t}_{e}\left(-\gamma_{e} u, \hat{q}\right)$ then

$$
\hat{z}=\hat{t}_{e} \hat{q} \text { and } \hat{c}=\left(1-\hat{t}_{e}\right) \gamma_{e}
$$

Now consider $Z, Q$ and $t_{e}$ as a sequences in (3.9.2) as follows $Z_{n}=\left(c_{n} u, z_{n}\right)$, $Q_{n}=\left(\overrightarrow{0}, q_{n}\right)$ and $t_{e}=t_{e n}$ so we have

$$
\overrightarrow{P Z_{n}}=t_{e n} \overrightarrow{P Q_{n}}
$$

requires that, $\left(\left(c_{n}-\gamma_{e}\right) u, z_{n}\right)=t_{e n}\left(-\gamma_{e} u, q_{n}\right)$ then

$$
z_{n}=t_{e n} q_{n} \text { and } c_{n}=\left(1-t_{e n}\right) \gamma_{h}
$$

Also consider $\hat{Z}, \hat{Q}$ and $\hat{t}_{e}$ as a sequences in (3.9.4) as follows $\hat{Z}_{n}=\left(\hat{c}_{n} u, \hat{z}_{n}\right)$, $\hat{Q}_{n}=\left(\overrightarrow{0}, \hat{q}_{n}\right)$ and $\hat{t}_{e}=\hat{t}_{e n}$ so we have

$$
\vec{P}_{n}=\hat{t}_{e n}{\overrightarrow{P \hat{Q}_{n}}}
$$


requires that, $\left(\left(\hat{c}_{n}-\gamma_{e}\right) u, \hat{z}_{n}\right)=\hat{t}_{e n}\left(-\gamma_{e} u, \hat{q}_{n}\right)$ then

$$
\hat{z}_{n}=\hat{t}_{e n} \hat{q}_{n} \text { and } \hat{c}_{n}=\left(1-\hat{t}_{e n}\right) \gamma_{e}
$$

Substitute $c=\left(1-t_{e}\right) \gamma_{e}$ in (3.9.1) we get

$$
\left(R e\left(\theta_{e} \overline{\left(1-t_{e}\right) \gamma_{e}}\right)\right)^{2}-d^{2}\left(\left(1-t_{e}\right) \gamma_{e}\right) \overline{\left(1-t_{e}\right) \gamma_{e}}-d^{2}\|z\|^{2}=d^{2} \theta_{e} \bar{\theta}_{e}-d^{4}
$$

use the fact that $1-t_{e}$ is a real number and simplify to get

$$
\left(1-t_{e}\right)^{2}\left(R e\left(\theta_{e} \bar{\gamma}_{e}\right)\right)^{2}-d^{2}\left(1-t_{e}\right)^{2} \gamma_{e} \bar{\gamma}_{e}-d^{2}\|z\|^{2}=d^{2} \theta_{e} \bar{\theta}_{e}-d^{4}
$$

rearrange terms

$$
\left(1-t_{e}\right)^{2}\left(\frac{\left(R e\left(\theta_{e} \bar{\gamma}_{e}\right)\right)^{2}}{d}-\gamma_{e} \bar{\gamma}_{e}\right)-\|z\|^{2}=\theta_{e} \bar{\theta}_{e}-d^{2}
$$

Let $\mathcal{D}=\frac{\left(\operatorname{Re}\left(\theta_{e} \bar{\gamma}_{e}\right)\right)^{2}}{d}-\gamma_{e} \bar{\gamma}_{e}$ and $\alpha_{e}=-\mathcal{D}+\theta_{e} \bar{\theta}_{e}-d^{2}$. We make two assumptions

1. $\mathcal{D}<0$

2. $\alpha_{e}<0$

We need the first assumption in order to satisfy the triangle inequality as following

$$
\left\|\left(c u+\theta_{e} u, z\right)\right\|_{H_{N}}+\left\|\left(c u-\theta_{e} u, z\right)\right\|_{H_{N}}=2 d
$$

and use a triangle with a vertices $(c u, z),\left(\theta_{e} u, \overrightarrow{0}\right)$ and $\left(-\theta_{e} u, \overrightarrow{0}\right)$ so the distances between $\left(\theta_{e}, \overrightarrow{0}\right)$ and $\left(-\theta_{e}, \overrightarrow{0}\right)$ is

$$
\begin{aligned}
\left\|\left(\theta_{e}, \overrightarrow{0}\right)-\left(-\theta_{e}, \overrightarrow{0}\right)\right\|_{H_{N}} & =\left\|\left(2 \theta_{e} u, \overrightarrow{0}\right)\right\|_{H_{N}}=\sqrt{\left\langle 2 \theta_{e} u, 2 \theta_{e} u\right\rangle} \\
& =\sqrt{2 \theta_{h} \overline{2 \theta}_{h}\langle u, u\rangle}=\sqrt{4 \theta_{e} \bar{\theta}_{e}}=2 \sqrt{\theta_{e} \overline{\theta_{e}}}=2\left|\theta_{e}\right|
\end{aligned}
$$

Use the fact that the sum of the lengths of any two sides of a triangle is greater then the length of the third side, so from (3.9.11) we have

$$
\left\|\left(c u+\theta_{e} u, z\right)\right\|_{H_{N}}+\left\|\left(c u-\theta_{e} u, z\right)\right\|_{H_{N}}>2\left|\theta_{e}\right|
$$


by (3.9.10) we have

$$
2 d>2\left|\theta_{e}\right|
$$

then we divide each side by 2 we get $\left|\theta_{e}\right|<d$ We know that any complex number can be considered as polar form. Let $\gamma_{e}=\rho_{1} e^{i \mu}$ and $\theta_{e}=\rho_{2} e^{i \nu}$ Since $\gamma_{e}$ and $\theta_{e}$ are in the Same direction so $\mu=\nu$ therefore

$$
\mathcal{D}=\left(\frac{\operatorname{Re}\left(\theta_{e} \bar{\gamma}_{e}\right)}{d}\right)^{2}-\gamma_{e} \bar{\gamma}_{e}=\left(\frac{\rho_{1} \rho_{2} \cos (\mu-\nu)}{d}\right)^{2}-\rho_{1}^{2}=\frac{\rho_{1}^{2} \rho_{2}^{2}}{d^{2}}-\rho_{1}^{2}
$$

since $\left|\theta_{e}\right|<d$ then $\rho_{1}^{2}<d^{2}$ therefore

$$
\mathcal{D}=\frac{\rho_{1}^{2} \rho_{2}^{2}}{d^{2}}-\rho_{1}^{2}=\rho_{1}^{2}\left(\frac{\rho_{1}^{2}}{d^{2}}-1\right)<0 .
$$

We must assume the second assumption in order to avoid complex values for $t_{e}$ for large $\|q\|$

$$
-\mathcal{D} t_{e}^{2}+2 \mathcal{D} t_{e}+\|z\|^{2}-\mathcal{D}+\theta_{e} \bar{\theta}_{e}-d^{2}=0
$$

or

$$
-\mathcal{D} t_{e}^{2}+2 \mathcal{D} t_{e}+\|z\|^{2}+\alpha_{e}=0
$$

solve for $t_{e}$ to obtain

$$
\begin{aligned}
t_{e} & =\frac{-2 \mathcal{D} \pm \sqrt{4 \mathcal{D}^{2}-4(-\mathcal{D})\left(\|z\|^{2}+\alpha_{e}\right)}}{-2 \mathcal{D}} \\
& =\frac{-2 \mathcal{D} \pm \sqrt{4 \mathcal{D}^{2}-4(-\mathcal{D})\left(\|z\|^{2}-\mathcal{D}+\theta_{e} \bar{\theta}_{e}-d^{2}\right)}}{-2 \mathcal{D}} \\
& =\frac{-2 \mathcal{D} \pm \sqrt{4 \mathcal{D}\left(\|z\|^{2}+\theta_{e} \bar{\theta}_{e}-d^{2}\right)}}{-2 \mathcal{D}}=1 \pm \frac{\sqrt{\mathcal{D}\left(\|z\|^{2}+\theta_{e} \bar{\theta}_{e}-d^{2}\right)}}{-\mathcal{D}}
\end{aligned}
$$

we always choose

$$
\begin{aligned}
t_{e} & =1-\frac{\sqrt{\mathcal{D}\left(\|z\|^{2}+\theta_{e} \bar{\theta}_{e}-d^{2}\right)}}{-\mathcal{D}} \\
& =1+\frac{\sqrt{\mathcal{D}\left(\|z\|^{2}+\theta_{e} \bar{\theta}_{e}-d^{2}\right)}}{\mathcal{D}}
\end{aligned}
$$


We want to express $t_{e}$ as a function of $q$, from (3.9.12) and use the fact $z=t q$ to obtain

$$
-\mathcal{D} t_{e}^{2}+2 \mathcal{D} t_{e}+\|t q\|^{2}+\alpha_{e}=0
$$

or

$$
\left(\|q\|^{2}-\mathcal{D}\right) t_{e}^{2}+2 \mathcal{D} t_{e}+\alpha_{e}=0
$$

since $\|q\|^{2}-\mathcal{D}>0$ and $\alpha_{e}<0$ then the equation (3.9.14) has one root negative and one positive. Solve for $t_{e}$ to obtain

$$
\begin{aligned}
t_{e} & =\frac{-2 \mathcal{D} \pm \sqrt{4 \mathcal{D}^{2}-4\left(\|q\|^{2}-\mathcal{D}\right) \alpha_{e}}}{2\left(\|q\|^{2}-\mathcal{D}\right)} \\
& =\frac{-\mathcal{D} \pm \sqrt{\mathcal{D}^{2}-\left(\|q\|^{2}-\mathcal{D}\right) \alpha_{e}}}{\|q\|^{2}-\mathcal{D}}
\end{aligned}
$$

we always choose

$$
t_{e}=\frac{-\mathcal{D}+\sqrt{\mathcal{D}^{2}-\left(\|q\|^{2}-\mathcal{D}\right) \alpha_{e}}}{\|q\|^{2}-\mathcal{D}}>0
$$

Assume $\mathcal{E}=\mathcal{D}^{2}-\left(\|q\|^{2}-\mathcal{D}\right) \alpha_{e}$, thus

$$
t_{e}=\frac{-\mathcal{D}+\sqrt{\mathcal{E}}}{\|q\|^{2}-\mathcal{D}}
$$

Repeat the steps above with the following points $\hat{Z}=(\hat{c} u, \hat{z})$ and $\hat{Q}=(\overrightarrow{0}, \hat{q})$, also consider

$$
\left\|(\hat{c} u, \hat{z})-\left(-\theta_{e} u, \overrightarrow{0}\right)\right\|_{H_{N}}+\left\|(\hat{c} u, \hat{z})-\left(\theta_{e} u, \overrightarrow{0}\right)\right\|_{H_{N}}=2 d,
$$

then by similar arguments we get

$$
\hat{t}_{e}=\frac{-\mathcal{D}+\sqrt{\hat{\mathcal{E}}}}{\|\hat{q}\|^{2}-\mathcal{D}}
$$

where

$$
\hat{\mathcal{E}}=\mathcal{D}^{2}-\left(\|\hat{q}\|^{2}-\mathcal{D}\right) \alpha_{e}
$$


When $\alpha_{e} \neq 0$ we have

$$
\begin{aligned}
t_{e} & =\frac{-\mathcal{D}+\sqrt{\mathcal{E}}}{\|q\|^{2}-\mathcal{D}}=\frac{(\sqrt{\mathcal{E}}-\mathcal{D})(\sqrt{\mathcal{E}}+\mathcal{D})}{\left(\|q\|^{2}-\mathcal{D}\right)(\sqrt{\mathcal{E}}+\mathcal{D})}=\frac{\mathcal{E}-\mathcal{D}^{2}}{\left(\|q\|^{2}-\mathcal{D}\right)(\sqrt{\mathcal{E}}+\mathcal{D})} \\
& =\frac{\mathcal{D}^{2}-\left(\|q\|^{2}-\mathcal{D}\right) \alpha_{e}-\mathcal{D}^{2}}{\left(\|q\|^{2}-\mathcal{D}\right)(\sqrt{\mathcal{E}}+\mathcal{D})}=\frac{-\left(\|q\|^{2}-\mathcal{D}\right) \alpha_{e}}{\left(\|q\|^{2}-\mathcal{D}\right)(\sqrt{\mathcal{E}}+\mathcal{D})} \\
& =\frac{-\alpha_{e}}{\sqrt{\mathcal{E}}+\mathcal{D}}
\end{aligned}
$$

Similar we can show that

$$
\hat{t}_{e}=\frac{-\alpha_{e}}{\sqrt{\hat{\mathcal{E}}}+\mathcal{D}}
$$

When $\alpha_{e}=0$ we have

$$
t_{e_{0}}=\frac{-2 \mathcal{D}_{0}}{\|q\|^{2}-\mathcal{D}_{0}}
$$

Proposition 3.9.1 if $0 \neq\left\|q_{n}\right\| \longrightarrow \infty$ then $t_{s n} \longrightarrow 0$

Proposition 3.9.2 Consider a sequences $t_{\text {en }}$ and $q_{n} \neq 0$ subject to (3.9.7) then we have

$$
t_{\text {en }} \sim \frac{\sqrt{-\alpha_{e}}}{\left\|q_{n}\right\|} \text { as } n \longrightarrow \infty
$$

and

$$
t_{e n} q_{n} \sim \sqrt{-\alpha_{e}} U_{n} \quad \text { as } n \longrightarrow \infty
$$

where

$$
U_{n}=\frac{q_{n}}{\left\|q_{n}\right\|}
$$

Proof Since

$$
\mathcal{E}_{n}=\mathcal{D}^{2}-\left(\left\|q_{n}\right\|^{2}-\mathcal{D}\right) \alpha_{e}=-\alpha_{e}\left\|q_{n}\right\|^{2}\left(1+\frac{\mathcal{D}^{2}+\mathcal{D} \alpha_{e}}{-\alpha_{e}\left\|q_{n}\right\|^{2}}\right)
$$

Thus

$$
\sqrt{\mathcal{E}_{n}} \sim\left\|q_{n}\right\| \sqrt{-\alpha_{e}} \text { as } n \longrightarrow \infty
$$


therefore

$$
t_{e} \sim \frac{-\mathcal{D}+\left\|q_{n}\right\| \sqrt{-\alpha_{e}}}{\left\|q_{n}\right\|^{2}-\mathcal{D}}=\frac{\sqrt{-\alpha_{e}}}{\left\|q_{n}\right\|}\left(\frac{1+\frac{-\mathcal{D}}{\left\|q_{n}\right\| \sqrt{-\alpha_{e}}}}{1+\frac{-\mathcal{D}}{\left\|q_{n}\right\|^{2}}}\right) \sim \frac{\sqrt{-\alpha_{e}}}{\left\|q_{n}\right\|} \text { as } n \longrightarrow \infty
$$

Also

$$
t_{e n} q_{n} \sim \frac{\sqrt{-\alpha_{e}}}{\left\|q_{n}\right\|} q_{n}=\sqrt{-\alpha_{e}} U_{n} \text { as } n \longrightarrow \infty
$$

Proposition 3.9.3 Consider a sequences $\hat{t}_{\text {en }}$ and $\hat{q}_{n} \neq 0$ subject to (3.9.9) then we have

$$
\hat{t}_{e n} \sim \frac{\sqrt{-\alpha_{e}}}{\left\|\hat{q}_{n}\right\|} \text { as } n \longrightarrow \infty
$$

and

$$
\hat{t}_{e n} \hat{q}_{n} \sim \sqrt{-\alpha_{e}} \hat{U}_{n} \text { as } n \longrightarrow \infty
$$

where

$$
\hat{U}_{n}=\frac{\hat{q}_{n}}{\left\|\hat{q}_{n}\right\|}
$$




\section{Chapter 4}

\section{A General Metric Induced}

\subsection{General Theorems}

Each family of boundizations can now be used to induce a particular metric. We introduce two types of metrics, one that is a sum of three terms, called an extended metric. The other metric, that is a sum of two terms, is called a projection metric. These definitions are motivated by a "Euclidean" distance and are elaborated upon in the sequel. It is noteworthy that our definitions of the two type of metrics apply to a Hilbert space and to a Banach space as well. However, a detailed analysis of the equivalence, and suprisingly, of the non equivalence among some of these metrics, is possible only in a Hilbert space thanks to its inner product property.

We begin with a general metric. Denote by $\mathcal{G}_{g}$ the a bijection mapping that matches each point $\left(\overrightarrow{0}, q_{n}\right) \in \mathbb{U} \mathbb{E} \mathbb{H}$ with a point $Z$ on a given surface

Definition 4.1.1 Consider a general surface subject to (3.4.1), then we define a mapping $\mathcal{G}_{g}\left(\left(\overrightarrow{0}, q_{n}\right)\right)$ from $\mathbb{U} \mathbb{E} \mathbb{H}$ into a general surface as follows

$$
\mathcal{G}_{g}\left(\left(\overrightarrow{0}, q_{n}\right)\right)= \begin{cases}\left(c_{n} u, t_{g n} q_{n}\right)=\left(\left(1-t_{g n}\right) \gamma_{g} u, t_{g n} q_{n}\right) & \text { if } q_{n} \in \mathbb{H} \\ \left(\gamma_{g} u, \psi(\infty) U\right)=\left(\gamma_{g} u, \sqrt{-\alpha_{g}} U\right) & \text { if } q_{n}=\infty U\end{cases}
$$

Define by $D_{g}\{Z, \hat{Z}\}=\|Z-\hat{Z}\|_{H_{N}}$ the distance between $(\overrightarrow{0}, q)$ and $(\overrightarrow{0}, \hat{q})$ in 
$\mathbb{U E H}$. Namely,

$$
\begin{aligned}
& D_{g}^{2}\{(\overrightarrow{0}, q),(\overrightarrow{0}, \hat{q})\} \\
& =\left\|((c-\hat{c}) u),\left(t_{g} q-\hat{t}_{g} \hat{q}\right)\right\|_{H_{N}}^{2} \\
& =\left\langle\left((c-\hat{c}) u, t_{g} q-\hat{t}_{g} \hat{q}\right),\left((c-\hat{c}) u, t_{g} q-\hat{t}_{g} \hat{q}\right)\right\rangle \\
& =\left\langle\left(\left(\hat{t}_{g}-t_{g}\right) \gamma_{g} u, t_{g} q-\hat{t}_{g} \hat{q}\right),\left(\left(\hat{t}_{g}-t_{g}\right) \gamma_{g} u, t_{g} q-\hat{t}_{g} \hat{q}\right)\right\rangle \\
& =\left(\hat{t}_{g}-t_{g}\right) \overline{\left.\hat{t}_{g}-t_{g}\right)} \gamma_{g} \bar{\gamma}_{g}\langle u, u\rangle+\left\langle t_{g} q-\hat{t}_{g} \hat{q}, t_{g} q-\hat{t}_{g} \hat{q}\right\rangle \\
& =\left(\hat{t}_{g}-t_{g}\right)^{2} \gamma_{g} \bar{\gamma}_{g}+\left\|t_{g} q-\hat{t}_{g} \hat{q}\right\|^{2} .
\end{aligned}
$$

Remark In what follows we elaborate on potentially indeterminate forms of $t_{g}(\|q\|) q$ and $t_{g}(\|\hat{q}\|) \hat{q}$ that may occur in definition 4.1.1 for certain $q, \hat{q} \in$ $\mathbb{U E H}$. It is shown in the following proposition that indeterminate forms as $0 \infty U$ can be made to be well defined. The reader should keep in mind section 3.1 discussing normalized boundizations.

Remark In a Banach space we define by $D_{g}\{Z, \hat{Z}\}=\|Z-\hat{Z}\|_{B_{N}}$ the distance between $(\overrightarrow{0}, q)$ and $(\overrightarrow{0}, \hat{q})$ in $\mathbb{U E B}$ as $\sqrt{\left(\hat{t}_{g}-t_{g}\right)^{2} \gamma_{g} \bar{\gamma}_{g}+\left\|t_{g} q-\hat{t}_{g} \hat{q}\right\|^{2}}$. This is the extended metric. The fact that in a Hilbert space $D_{g}^{2}\{(\overrightarrow{0}, q),(\overrightarrow{0}, \hat{q})\}$ can be written as a sum of three terms is due to the following.

Proposition 4.1.1 Suppose $(\overrightarrow{0}, q)$ and $(\overrightarrow{0}, \hat{q})$ are any two points corresponding to two elements in $\mathbb{U E H}$ with $q \neq \infty U_{1}$ and $\hat{q} \neq \infty U_{2}$. Then the distance between these points is given by,

$$
\begin{aligned}
& D_{g}^{2}\{(\overrightarrow{0}, q),(\overrightarrow{0}, \hat{q})\} \\
& =\left(\hat{t}_{g}-t_{g}\right)^{2} \gamma_{g} \bar{\gamma}_{g}+\left(t_{g}\|q\|-\hat{t}_{g}\|\hat{q}\|\right)^{2}+\hat{t}_{g} t_{g}(2\|q\|\|\hat{q}\|-\langle\hat{q}, q\rangle-\langle q, \hat{q}\rangle)
\end{aligned}
$$




\section{Proof}

$$
\begin{aligned}
& D_{g}^{2}\{(\overrightarrow{0}, q),(\overrightarrow{0}, \hat{q})\} \\
& =\left(\hat{t}_{g}-t_{g}\right)^{2} \gamma_{g} \bar{\gamma}_{g}+\left\|t_{g} q-\hat{t}_{g} \hat{q}\right\|^{2} \\
& =\left(\hat{t}_{g}-t_{g}\right)^{2} \gamma_{g} \bar{\gamma}_{g}+\left\langle t_{g} q, t_{g} q\right\rangle+\left\langle-\hat{t}_{g} \hat{q},-\hat{t}_{g} \hat{q}\right\rangle+\left\langle-\hat{t} g \hat{q}, t_{g} q\right\rangle+\left\langle t_{g} q,-\hat{t}_{g} \hat{q}\right\rangle \\
& =\left(\hat{t}_{g}-t_{g}\right)^{2} \gamma_{g} \bar{\gamma}_{g}+t_{g}^{2}\|q\|^{2}+\hat{t}_{g}^{2}\|\hat{q}\|^{2}-\hat{t}_{g} t_{g}[\langle\hat{q}, q\rangle+\langle q, \hat{q}\rangle] \\
& =\left(\hat{t}_{g}-t_{g}\right)^{2} \gamma_{g} \bar{\gamma}_{g}+t_{g}^{2}\|q\|^{2}+\hat{t}_{g}^{2}\|\hat{q}\|^{2} \\
& -2 \hat{t}_{g} t_{g}\|\hat{q}\| q\left\|+2 \hat{t}_{g} t_{g}\right\| \hat{q}\|q\|-\hat{t}_{g} t_{g}(\langle\hat{q}, q\rangle+\langle q, \hat{q}\rangle) \\
& =\left(\hat{t}_{g}-t_{g}\right)^{2} \gamma_{g} \bar{\gamma}_{g}+\left(t_{g}\|q\|-\hat{t}_{g}\|\hat{q}\|\right)^{2}+\hat{t}_{g} t_{g}(2\|q\|\|\hat{q}\|-\langle\hat{q}, q\rangle-\langle q, \hat{q}\rangle) \\
& =\left(\hat{t}_{g}-t_{g}\right)^{2} \gamma_{g} \bar{\gamma}_{g}+\left(t_{g}\|q\|-\hat{t}_{g}\|\hat{q}\|\right)^{2}+\hat{t}_{g}\|\hat{q}\| t_{g}\|q\|\left(2-\left\langle U_{2}, U_{1}\right\rangle-\left\langle U_{1}, U_{2}\right\rangle\right)
\end{aligned}
$$

Similarly we can find the distance between $(\overrightarrow{0}, \infty U)$ and $(\overrightarrow{0}, q)$

Proposition 4.1.2 Consider $\left(\overrightarrow{0}, \infty U_{2}\right)$ and $(\overrightarrow{0}, q)$ are two points corresponding to two elements in $\mathbb{U E H}$ with $0 \neq q \neq \infty U_{1}$ and $\hat{q}=\infty U_{2}$. Then the distance between these points is given by

$$
\begin{aligned}
& D_{g}^{2}\left\{(\overrightarrow{0}, q),\left(\overrightarrow{0}, \infty U_{2}\right)\right\} \\
& =t_{g}^{2} \gamma_{g} \bar{\gamma}_{g}+\left(\psi(\|q\|)-\sqrt{-\alpha_{g}}\right)^{2}+\psi(\|q\|) \sqrt{-\alpha_{g}}\left(2-\left\langle U_{2}, U_{1}\right\rangle-\left\langle U_{1}, U_{2}\right\rangle\right)
\end{aligned}
$$

where $U_{1}=\frac{q}{\|q\|}$ and $U_{2}=\frac{\hat{q}}{\|\hat{q}\|}$

\section{Proof}

$$
\begin{aligned}
& D_{g}^{2}\left\{(\overrightarrow{0}, q),\left(\overrightarrow{0}, \infty U_{2}\right)\right\} \\
& =\left\|\left(c-\gamma_{g}\right) u, t_{g} q-\sqrt{-\alpha_{g}} U_{2}\right\|_{H_{N}}^{2} \\
& =\left\langle\left(\left(c-\gamma_{g}\right) u, t_{g} q-\sqrt{-\alpha_{g}} U_{2}\right),\left(\left(c-\gamma_{g}\right) u, t_{g} q-\sqrt{-\alpha_{g}} U_{2}\right)\right\rangle_{H_{N}} \\
& =\left\langle\left(-t_{g} \gamma_{g} u, t_{g} q-\sqrt{-\alpha_{g}} U_{2}\right),\left(-t_{g} \gamma_{g} u, t_{g} q-\sqrt{-\alpha_{g}} U_{2}\right)\right\rangle_{H_{N}} \\
& =\left(-t_{g}\right) \overline{(-t)_{g}} \gamma_{g} \bar{\gamma}_{g}\langle u, u\rangle+\left\langle t_{g} q-\sqrt{-\alpha_{g}} U_{2}, t_{g} q-\sqrt{-\alpha_{g}} U_{2}\right\rangle \\
& =t_{g}^{2} \gamma_{g} \bar{\gamma}_{g}+\left\langle t_{g} q, t_{g} q\right\rangle-\alpha_{g}\left\langle U_{2}, U_{2}\right\rangle+\left\langle-\sqrt{-\alpha_{g}} U_{2}, t_{g} q\right\rangle+\left\langle t_{g} q,-\sqrt{-\alpha_{g}} U_{2}\right\rangle \\
& =t_{g}^{2} \gamma_{g} \bar{\gamma}_{g}+t_{g}^{2}\langle q, q\rangle-\alpha_{g}-t_{g} \sqrt{-\alpha_{g}}\left(\left\langle U_{2}, q\right\rangle+\left\langle q, U_{2}\right\rangle\right) \\
& =t_{g}^{2} \gamma_{g} \bar{\gamma}_{g}+t_{g}^{2}\|q\|^{2}-\alpha_{g}-2 t_{g}\|q\| \sqrt{-\alpha_{g}} \\
& +2 t_{g}\|q\| \sqrt{-\alpha_{g}}-t_{g} \sqrt{-\alpha_{g}}\left(\left\langle U_{2}, q\right\rangle+\left\langle q, U_{2}\right\rangle\right) \\
& =t_{g}^{2} \gamma_{g} \bar{\gamma}_{g}+\left(t_{g}\|q\|-\sqrt{-\alpha_{g}}\right)^{2}+t_{g} \sqrt{-\alpha_{g}}\left(2\|q\|-\left\langle U_{2}, q\right\rangle-\left\langle q, U_{2}\right\rangle\right) \\
& =t_{g}^{2} \gamma_{g} \bar{\gamma}_{g}+\left(\psi(\|q\|)-\sqrt{-\alpha_{g}}\right)^{2}+\psi(\|q\|) \sqrt{-\alpha_{g}}\left(2-\left\langle U_{2}, U_{1}\right\rangle-\left\langle U_{1}, U_{2}\right\rangle\right)
\end{aligned}
$$


Finally the distance between $\left(\overrightarrow{0}, \infty U_{1}\right)$ and $\left(\overrightarrow{0}, \infty U_{2}\right)$ is given by

Proposition 4.1.3 Assume that $\left(\overrightarrow{0}, \infty U_{1}\right)$ and $\left(\overrightarrow{0}, \infty U_{2}\right)$ are any two points corresponding to two elements in $\mathbb{U} \mathbb{E} \mathbb{H}$ with $q=\infty U_{1}$ and $\hat{q}=\infty U_{2}$. Then the distance between these points is given by

$$
D_{g}^{2}\left\{\left(\overrightarrow{0}, \infty U_{1}\right),\left(\overrightarrow{0}, \infty U_{2}\right)\right\}=-\alpha_{g}\left\|U_{1}-U_{2}\right\|^{2}
$$

\section{Proof}

$$
\begin{aligned}
D_{g}^{2}\left\{\left(\overrightarrow{0}, \infty U_{1}\right),\left(\overrightarrow{0}, \infty U_{2}\right)\right\} & =\left\|\left(\overrightarrow{0}, \sqrt{-\alpha_{g}}\left(U_{1}-U_{2}\right)\right)\right\|_{H_{N}}^{2} \\
& =\left\langle\sqrt{-\alpha_{g}}\left(U_{1}-U_{2}\right), \sqrt{-\alpha_{g}}\left(U_{1}-U_{2}\right)\right\rangle \\
& =-\alpha_{g}\left\|U_{1}-U_{2}\right\|^{2}
\end{aligned}
$$

Theorem 4.1.4 The distance between any two points in $\mathbb{U} \mathbb{E} \mathbb{H}$ defines an extended metric and a projection metric as follows

1. Let $(\overrightarrow{0}, q)$ and $(\overrightarrow{0}, \hat{q})$ such that $q, \hat{q} \in \mathbb{H}$ then the extended metric is

$$
\begin{aligned}
& D_{g}^{2}\{(\overrightarrow{0}, q),(\overrightarrow{0}, \hat{q})\} \\
& =\left(\hat{t}_{g}-t_{g}\right)^{2} \gamma_{g} \bar{\gamma}_{g}+\left(t_{g}\|q\|-\hat{t}_{g}\|\hat{q}\|\right)^{2}+\hat{t}_{g} t_{g}(2\|q\|\|\hat{q}\|-\langle\hat{q}, q\rangle-\langle q, \hat{q}\rangle)
\end{aligned}
$$

and its projection metric is

$$
D_{g}^{2}\{(\overrightarrow{0}, q),(\overrightarrow{0}, \hat{q})\}=\left(t_{g}\|q\|-\hat{t}_{g}\|\hat{q}\|\right)^{2}+\hat{t}_{g} t_{g}(2\|q\|\|\hat{q}\|-\langle\hat{q}, q\rangle-\langle q, \hat{q}\rangle)
$$

2. Let $(\overrightarrow{0}, q),\left(\overrightarrow{0}, \infty U_{2}\right)$ such that $q \in \mathbb{H}$ then the extended metric is

$$
\begin{aligned}
& D_{g}^{2}\left\{(\overrightarrow{0}, q),\left(\overrightarrow{0}, \infty U_{2}\right)\right\} \\
& =t_{g}^{2} \gamma_{g} \bar{\gamma}_{g}+\left(t_{g}\|q\|-\sqrt{-\alpha_{g}}\right)^{2}+t_{g} \sqrt{-\alpha_{g}}\left(2\|q\|-\left\langle U_{2}, q\right\rangle-\left\langle q, U_{2}\right\rangle\right) \\
& =t_{g}^{2} \gamma_{g} \bar{\gamma}_{g}+\left(\psi(\|q\|)-\sqrt{-\alpha_{g}}\right)^{2}+\psi(\|q\|) \sqrt{-\alpha_{g}}\left(2-\left\langle U_{2}, q\right\rangle-\left\langle q, U_{2}\right\rangle\right)
\end{aligned}
$$

and its projection metric is

$$
\begin{aligned}
& D_{g}^{2}\left\{(\overrightarrow{0}, q),\left(\overrightarrow{0}, \infty U_{2}\right)\right\} \\
& =\left(t_{g}\|q\|-\sqrt{-\alpha_{g}}\right)^{2}+t_{g} \sqrt{-\alpha_{g}}\left(2\|q\|-\left\langle U_{2}, q\right\rangle-\left\langle q, U_{2}\right\rangle\right) \\
& =\left(\psi(\|q\|)-\sqrt{-\alpha_{g}}\right)^{2}+\psi(\|q\|) \sqrt{-\alpha_{g}}\left(2-\left\langle U_{2}, q\right\rangle-\left\langle q, U_{2}\right\rangle\right)
\end{aligned}
$$


3. Let $\left(\overrightarrow{0}, \infty U_{1}\right),\left(\overrightarrow{0}, \infty U_{2}\right)$ then

$$
D_{g}^{2}\left\{\left(\overrightarrow{0}, \infty U_{1}\right),\left(\overrightarrow{0}, \infty U_{2}\right)\right\}=-\alpha_{g}\left\|U_{1}-U_{2}\right\|^{2}
$$

where $U_{1}$ is a unit vector of $q$ and $U_{2}$ is a unit vector of $\hat{q}$

Moreover, the metric is continuous in $\mathbb{U E H}$ as follows.

$$
D_{g}^{2}\left\{(\overrightarrow{0}, q),\left(\overrightarrow{0}, \infty U_{2}\right)\right\}=\lim _{\left\|\hat{q}_{n}\right\| \longrightarrow \infty} D_{g}^{2}\left\{(\overrightarrow{0}, q),\left(\overrightarrow{0}, \hat{q}_{n}\right)\right\}
$$

and

$$
D_{g}^{2}\left\{\left(\overrightarrow{0}, \infty U_{1}\right),\left(\overrightarrow{0}, \infty U_{2}\right)\right\}=\lim _{\substack{\left\|q_{n}\right\| \longrightarrow \infty \\\left\|\hat{q}_{n}\right\| \rightarrow \infty}} D_{g}^{2}\left\{\left(\overrightarrow{0}, q_{n}\right),\left(\overrightarrow{0}, \hat{q}_{n}\right)\right\}
$$

Proof The first two conditions in the definition of a metric are obviously satisfied, namely for positivity $D_{g}$ is nonnegative number $D_{g}\{(\overrightarrow{0}, q),(\overrightarrow{0}, \hat{q})\} \geq$ 0 and by $D_{g}$ only be equal to zero if and only if when $q=\hat{q}$ or when $\|q\|=\|\hat{q}\|$.

$$
D_{g}\{(\overrightarrow{0}, q),(\overrightarrow{0}, \hat{q})\}=0 \text { if and only if } q=\hat{q} \text { and then }\|q\|=\|\hat{q}\| .
$$

And for symmetry we have

$$
D_{g}\{(\overrightarrow{0}, q),(\overrightarrow{0}, \hat{q})\}=\|(\overrightarrow{0}, q)-(\overrightarrow{0}, \hat{q})\|_{\mathbb{H}_{N}}=\|(\overrightarrow{0}, \hat{q})-(\overrightarrow{0}, q)\|_{H_{N}}=D_{g}\{(\overrightarrow{0}, \hat{q}),(\overrightarrow{0}, q)\}
$$

To prove the triangle inequality, observe for any third point in $\mathbb{U E H}(\overrightarrow{0}, q)$, $(\overrightarrow{0}, \hat{q})$ and $(\overrightarrow{0}, \tilde{q})$ we have

$$
\begin{aligned}
D_{g}\{(\overrightarrow{0}, q),(\overrightarrow{0}, \hat{q})\} & =\|(\overrightarrow{0}, q)-(\overrightarrow{0}, \hat{q})\|_{H_{N}} \\
& =\|(\overrightarrow{0}, q)-(\overrightarrow{0}, \tilde{q})+(\overrightarrow{0}, \tilde{q})-(\overrightarrow{0}, \hat{q})\|_{H_{N}} \\
& \leq\|(\overrightarrow{0}, q)-(\overrightarrow{0}, \tilde{q})\|_{H_{N}}+\|(\overrightarrow{0}, \tilde{q})-(\overrightarrow{0}, \hat{q})\|_{H_{N}} \\
& \leq D_{g}\{(\overrightarrow{0}, q),(\overrightarrow{0}, \tilde{q})\}+D_{g}\{(\overrightarrow{0}, \tilde{q}),(\overrightarrow{0}, \hat{q})\}
\end{aligned}
$$

Moreover,

$$
D_{g}^{2}\{(\overrightarrow{0}, q),(\overrightarrow{0}, \hat{q})\}=\left(\hat{t}_{g}-t_{g}\right)^{2} \gamma_{g} \bar{\gamma}_{g}+\left\langle t_{g} q-\hat{t}_{g} \hat{q}, t_{g} q-\hat{t}_{g} \hat{q}\right\rangle
$$

by (3.4.5) we have

$$
\lim _{q \longrightarrow \infty} \hat{t}_{g} \hat{q}=\sqrt{-\alpha_{g}} U_{2} \quad \text { as } \hat{q} \longrightarrow \infty
$$


and as we know that $\|\hat{q}\| \longrightarrow \infty$ then $\hat{t}_{g} \longrightarrow 0$, thus the equation (4.1.10) becomes

$$
D_{g}^{2}\left\{(\overrightarrow{0}, q),\left(\overrightarrow{0}, \infty U_{2}\right)\right\}=t_{g}^{2} \gamma_{g} \bar{\gamma}_{g}+\left\langle t_{g} q-\sqrt{-\alpha_{g}} U_{2}, t_{g} q-\sqrt{-\alpha_{g}} U_{2}\right\rangle
$$

therefore we get the equation (4.1.6). And when $\|q\| \longrightarrow \infty$ and $\|\hat{q}\| \longrightarrow \infty$ in the equation (4.1.4) we will get the equation (4.1.3) as follows. By (3.4.5) we have $t_{g n} q_{n} \sim \sqrt{-\alpha_{g}} U_{1}$ and $\hat{t}_{g n} \hat{q}_{n} \sim \sqrt{-\alpha_{g}} U_{2}$ as $n \longrightarrow \infty$ and by the definition 4.3.1 we have $t_{g} q=\sqrt{-\alpha_{g}} U_{1}$ and $\hat{t}_{g} \hat{q}=\sqrt{-\alpha_{g}} U_{2}$ also as we know when $\|q\| \longrightarrow \infty$ then $t_{g} \longrightarrow 0$ and when $\|\hat{q}\| \longrightarrow \infty$ then $\hat{t}_{g} \longrightarrow 0$ therefore the equation (4.1.10) becomes

$$
D_{s}^{2}\left\{\left(\overrightarrow{0}, \infty U_{2}\right),\left(\overrightarrow{0}, \infty U_{2}\right)\right\}=\left\langle\sqrt{-\alpha_{g}} U_{1}-\sqrt{-\alpha_{g}} U_{2}, \sqrt{-\alpha_{g}} U_{1}-\sqrt{-\alpha_{g}} U_{2}\right\rangle
$$

therefore we get (4.1.3)

Remark The distances and formulas that are derived above can be adapted in a straight forward manner to $\mathbb{U E \mathbb { B }}$ spaces excluding of course the formulas that utilize the representation

$\left\|\left(t_{g} q-\hat{t}_{g} \hat{q}\right)\right\|^{2}=\left(\psi(\|q\|)-\psi(\|\hat{q}\|)^{2}+\psi(\|\hat{q}\|) \psi(\|q\|)\left(2-\left\langle U_{2}, U_{1}\right\rangle-\left\langle U_{1}, U_{2}\right\rangle\right)\right.$

in which an inner is used.

\subsection{Approximating Schemes in Banach spaces via Boundizations}

Let $\mathbb{B}$ be a Banach space over $\mathbb{R}$ or $\mathbb{C}$. Let $q \in \mathbb{B}$ and let $q_{n}, n=1,2,3, \ldots$ be an approximating sequence to $q$ such that

$$
\lim _{n \rightarrow \infty}\left\|q-q_{n}\right\|=0
$$

An examination of text book in approximation theory See e.g. C.F.Dunkl and Yuan Xu [14], G. Freud [16] and W. Gautschi [18] reveal that in order to obtain an approximation sequence to $q$ one must assume an order of growth on $q$ that is manifested in the norm of $q$ being bounded. It is easy to bring examples of functions with singularities such as given at the beginning of section 1.1 and such as example (1.1.1) that will render such functions not amenable 
to any approximation scheme. This is a setting where boundizations come to the rescue. We desire to approximate in $\mathbb{U E \mathbb { B }}$ an element $\infty U$ or an element $q \in \mathbb{B}$ with an exceptional very large norm. To this end we consider first the element $z(q)=t(\|q\|) q$ if $q \in \mathbb{B}$ or $z=t(\|q\|)\|q\|[\|q\|]^{-1} q=\psi(\infty) U=L U$ if our element is $\infty U$. We then take an approximating sequence $z_{n} \in \mathbb{B}$ such that

$$
\lim _{n \rightarrow \infty}\left\|z-z_{n}\right\|=0
$$

Since $z(q)$ is a bijection we consider the unique sequence solution $b_{n}, n=$ $1,2, \ldots$ such that

$$
z_{n}=t\left(\left\|b_{n}\right\|\right)\left\|b_{n}\right\|\left[\left\|b_{n}\right\|\right]^{-1} b_{n}=\psi\left(\left\|b_{n}\right\|\right)\left[\left\|b_{n}\right\|\right]^{-1} b_{n}=q_{n} .
$$

The algorithm to solving now for $b_{n}, n=1,2, \ldots$ is as follows. First we compute the norm of $z_{n}$ by noticing from (4.2.3) that $\left\|z_{n}\right\|=\psi\left(\left\|b_{n}\right\|\right)$. This requires the solution of a scalar equation for the inverse function $\left\|b_{n}\right\|=$ $\psi^{-1}\left(\left\|z_{n}\right\|\right)$. It is also readily observed from (4.2.3) that the direction of $b_{n}$ is the same as the direction of $z_{n}$. By the continuity of $\Psi$ and its inverse we discover that $b_{n}=\psi^{-1}\left(\left\|z_{n}\right\|\right)\left[\left\|z_{n}\right\|\right]^{-1} z_{n}$ is the desired approximation to any element in $\mathbb{U E} \mathbb{B}$ except of course to the zero element in $\mathbb{B}$.

\subsubsection{Consistency of approximations in Banach Spaces}

We desire to know if a projection metric and if an extended metric on $\mathbb{U} \mathbb{E} \mathbb{H}$ , defined by (4.1.4) or (4.1.5), is consistent with (4.2.1). An answer to this question is provided by the next proposition.

Proposition 4.2.1 Let $q, q_{n} \in \mathbb{B}$ and let $\lim _{n \rightarrow \infty}\left\|q-q_{n}\right\|=0$. Then, there exists constants $C_{1}>0$ and $C_{2}>0$ such that for all $n$ in the projection metric we have

$$
\left|t_{g}(\|q\|) q-t_{g}\left(\left\|q_{n}\right\|\right) q_{n}\right| \leq C_{1}\left\|q-q_{n}\right\|
$$

and in the extended metric we have

$$
\begin{aligned}
& D_{g}\{(\overrightarrow{0}, q),(\overrightarrow{0}, \hat{q})\} \\
& =\sqrt{\left(t_{g}(\|q\|)-t_{g}\left(\left\|q_{n}\right\|\right)\right)^{2} \gamma_{g} \bar{\gamma}_{g}+\left(t_{g}(\|q\|) q-t_{g}\left(\left\|q_{n}\right\| q_{n}\right)^{2}\right.} \\
& \leq C_{2}\left\|q-q_{n}\right\| .
\end{aligned}
$$


Proof Notice first that $q \in \mathbb{B}$ together with (4.2.1) imply that there exist $M>0$ such that $\|q\| \leq M$ and $\left\|q_{n}\right\| \leq M$ for all $n$. Consider first the estimates

$$
\begin{aligned}
\left|t_{g}(\|q\|) q-t_{g}\left(\left\|q_{n}\right\|\right) q_{n}\right| & =\left|t_{g}(\|q\|)\left(q-q_{n}\right)+t_{g}(\|q\|) q_{n}-t_{g}\left(\left\|q_{n}\right\|\right) q_{n}\right| \\
& \leq\left|t_{g}(\|q\|)\left\|q-q_{n}\right\|\right|+\left|t_{g}(\|q\|)-t_{g_{1}}\left(\left\|q_{n}\right\|\right)\right|\left\|q_{n}\right\| \mid \\
& \leq t_{g}(\|q\|)\left\|q-q_{n}\right\|+M\left|t_{g}(\|q\|)-t_{g}\left(\left\|q_{n}\right\|\right)\right| .
\end{aligned}
$$

From the definition of $t_{g}(\|q\|)$ it follows that $\lim _{\|q\| \rightarrow \infty} t_{g}(\|q\|)=0$ and that $0 \leq t_{g}(\|q\|) \leq L$, for some finite $L>0$.Putting all these conclusions in (4.2.6) we obtain that

$$
\left|t_{g}(\|q\|) q-t_{g}\left(\left\|q_{n}\right\|\right) q_{n}\right| \leq L\left\|q-q_{n}\right\|+M M_{1}\left\|q-q_{n}\right\|,
$$

and the conclusion in (4.2.4) follows with $C_{1}=L+M M_{1}$. Notice that because $t_{g_{1}}(\|q\|)$ is Lipschitz we have

$$
\left(t_{g_{1}}(\|q\|)-t_{g_{1}}\left(\left\|q_{n}\right\|\right)\right)^{2} \gamma_{g_{1}} \bar{\gamma}_{g_{1}} \leq M_{1}^{2} \gamma_{g_{1}} \bar{\gamma}_{g_{1}}\left\|q-q_{n}\right\|^{2}
$$

Choose $C_{2}=\sqrt{C_{1}^{2}+M_{1}^{2} \gamma_{g} \bar{\gamma}_{g}}$ and the conclusion follows.

\subsubsection{Convergence in A General Surface Boundization}

In this section we use the extended metric to explain Convergence on a general surface Boundization.

Proposition 4.2.2 Consider $\rho_{n_{1}}, \rho_{n_{2}}$ to be sequences in $\mathbb{U E H}$ then For $c>0$ we have

$$
D_{g}^{2}\left\{\left(c \rho_{n_{1}}\right) U_{1},\left(\rho_{n_{2}}\right) U_{1}\right\} \longrightarrow 0 \text { as } n_{1}, n_{2} \longrightarrow \infty
$$

Proof By theorem 4.1.4 and the equation (2.3.2) we have as $n_{1}, n_{2} \longrightarrow \infty$

$$
D_{g}^{2}\left\{\left(\rho_{n_{1}}\right) U_{1},\left(\rho_{n_{2}}\right) U_{1}\right\} \text { implies that } D_{g}^{2}\left\{\infty U_{1}, \infty U_{1}\right\}=0
$$


Remark Proposition 4.2.2 explains why $\infty U$ with $\|U\|=1$ actually represents an equivalence class of objects of the form $\infty V$ with $\|V\| \neq 0$. Define the relation $\mathcal{R}$ so that $\left(\infty V_{1}\right) \mathcal{R}\left(\infty V_{2}\right),\left\|V_{1}\right\|\left\|V_{2}\right\| \neq 0$, if $\left\|V_{1}\right\|^{-1} V_{1}=$ $\left\|V_{2}\right\|^{-1} V_{2}$. Treader can easily verify that $\mathcal{R}$ is an equivalence relation. Proposition 4.2.2 justifies the facts that

$$
D^{2}\left[\left(0, \infty V_{1}\right),\left(0, \infty V_{2}\right)\right]=0
$$

by considering any two sequences $\rho_{n_{1}}, \rho_{n_{2}} \longrightarrow \infty$ as $n_{1}, n_{2} \longrightarrow \infty$.

Theorem 4.2.3 $U \mathbb{E} \mathbb{B}$ is a complete metric space in the extended and projection metrics.

Proof Let $\left\|q_{n}\right\|$ be a sequence such that $\left\|q_{n}\right\| \longrightarrow 0$ as $n \longrightarrow \infty$. Then by proposition 4.2 .1 we have

$$
D_{g}\{(\overrightarrow{0}, q),(\overrightarrow{0}, \hat{q})\} \leq C_{2}\left\|q-q_{n}\right\|
$$

for some fixed $C_{2}>0$. Therefore $\lim _{n \rightarrow \infty} q_{n}=\overrightarrow{0} \in \mathbb{U} \mathbb{E} \mathbb{B}$ Now consider a sequence $\left(\overrightarrow{0}, q_{n}\right), q_{n} \in \mathbb{U} \mathbb{E} \mathbb{B}$. We will show that if the sequence is Cauchy in the extended or projection metrics then there exist an element $h$ in $\mathbb{U E \mathbb { B }}$ such that $\lim _{n \rightarrow \infty}\left(\overrightarrow{0}, q_{n}\right)=(0, h)$. Suppose that $\left(0, q_{n}\right)$ is a Cauchy sequence then by definition we have

$$
D_{g}^{2}\left\{\left(\overrightarrow{0}, q_{n}\right),\left(\overrightarrow{0}, q_{m}\right)\right\} \longrightarrow 0 \text { as } n, m \longrightarrow \infty,
$$

where $D_{g}^{2}$ coincides with the extended metric. This implies that

$$
\|0 u-0 u\|+\left\|t\left(\left\|q_{n}\right\|\right) q_{n}-t\left(\left\|q_{m}\right\|\right) q_{m}\right\| \longrightarrow 0 .
$$

Assume without loss of generality that $q_{n} \neq 0$ and $q_{m} \neq 0$ then we have

$$
\left\|\psi\left(\left\|q_{n}\right\|\right) U_{n}-\psi\left(\left\|q_{m}\right\|\right) U_{m}\right\| \longrightarrow 0
$$

where $U_{n}=\frac{q_{n}}{\left\|q_{n}\right\|}$ and $U_{m}=\frac{q_{m}}{\left\|q_{m}\right\|}$. Since

$$
\left|\psi\left(\left\|q_{n}\right\|\right)\left\|U_{n}\right\|-\psi\left(\left\|q_{m}\right\|\right)\left\|U_{m}\right\|\right| \leq\left\|\psi\left(\left\|q_{n}\right\|\right) U_{n}-\psi\left(\left\|q_{m}\right\|\right) U_{m}\right\| \longrightarrow 0 .
$$

This mean, that

$$
\left|\psi\left(\left\|q_{n}\right\|\right)\left\|U_{n}\right\|-\psi\left(\left\|q_{m}\right\|\right)\left\|U_{m}\right\|\right| \longrightarrow 0
$$


which make $\psi\left(\left\|q_{n}\right\|\right)$ a Cauchy sequence in $\mathbb{R}$. Consequently, there exist $\psi_{0}$ such that $\left|\psi\left(\left\|q_{n}\right\|\right)-\psi_{0}\right| \longrightarrow 0$ as $n \longrightarrow \infty$. Therefore

$$
\begin{aligned}
& \left\|\psi\left(\left\|q_{n}\right\|\right) U_{n}-\psi\left(\left\|q_{m}\right\|\right) U_{m}\right\| \\
& =\left\|\left(\psi\left(\left\|q_{n}\right\|\right)-\psi_{0}\right) U_{n}-\left(\psi\left(\left\|q_{m}\right\|\right)-\psi_{0}\right) U_{m}+\psi_{0} U_{n}-\psi_{0} U_{m}\right\| .
\end{aligned}
$$

We will show that $U_{n}$ is also a Cauchy sequence. By equation (4.2.9) and the triangle inequality we have

$$
\begin{aligned}
& \left\|\psi_{0}\left(U_{n}-U_{m}\right)\right\|-\left|\left(\psi\left(\left\|q_{n}\right\|\right)-\psi_{0}\right)\right|\left\|U_{n}\right\|-\left|\left(\psi\left(\left\|q_{m}\right\|\right)-\psi_{0}\right)\right|\left\|U_{m}\right\| \\
& \leq\left\|\psi\left(\left\|q_{n}\right\|\right) U_{n}-\psi\left(\left\|q_{m}\right\|\right) U_{m}\right\| .
\end{aligned}
$$

Therefore

$$
\begin{aligned}
& \psi_{0}\left\|U_{n}-U_{m}\right\| \leq\left|\left(\psi\left(\left\|q_{n}\right\|\right)-\psi_{0}\right)\right|\left\|U_{n}\right\|+\left|\left(\psi\left(\left\|q_{m}\right\|\right)-\psi_{0}\right)\right|\left\|U_{m}\right\| \\
& +\left\|\psi\left(\left\|q_{n}\right\|\right) U_{n}-\psi\left(\left\|q_{m}\right\|\right) U_{m}\right\| .
\end{aligned}
$$

Since

$$
\begin{aligned}
& \left|\left(\psi\left(\left\|q_{n}\right\|\right)-\psi_{0}\right)\right|\left\|U_{n}\right\| \longrightarrow 0,\left|\left(\psi\left(\left\|q_{m}\right\|\right)-\psi_{0}\right)\right|\left\|U_{m}\right\| \longrightarrow 0 \\
& \text { and }\left\|\psi\left(\left\|q_{n}\right\|\right) U_{n}-\psi\left(\left\|q_{m}\right\|\right) U_{m}\right\| \longrightarrow 0 \quad n, m \longrightarrow \infty
\end{aligned}
$$

and $0<\psi_{0} \leq L$, then

$$
\left\|U_{q_{n}}-U_{q_{m}}\right\| \longrightarrow 0 \quad n, m \longrightarrow \infty
$$

Thus $U_{q_{n}}$ is a Cauchy sequence in a Banach space $\mathbb{B}$ therefore it is convergent to an element $\hat{h}$ in $\mathbb{B}$ so

$$
\lim _{n \longrightarrow \infty} U_{q_{n}}=\hat{h} .
$$

Hence

$$
\lim _{n \longrightarrow \infty} q_{n}=\psi_{0} \hat{h}=h
$$

which proves our statement. 


\subsubsection{Examples of A General Surface Boundization}

To illustrate the concept of a general surface boundization, let us take a look at some examples from functional analysis. If any one had a good functional analysis course, then a lot (though not all) of the examples below should look somewhat familiar, however what we did is a new and not familiar. We give six examples.

Example 4.2.1 Assume $\mathbb{H}=\mathbb{C}^{n}$ then

$$
D_{g}^{2}\left\{\left(\overrightarrow{0}, \infty U_{1}\right),\left(\overrightarrow{0}, \infty U_{2}\right)\right\}=-\alpha_{g}\left(U_{1}-U_{2}\right)^{\star}\left(U_{1}-U_{2}\right)
$$

Example 4.2.2 Assume $\mathbb{H}=\mathbb{R}^{n}$ then

$$
D_{g}^{2}\left\{\left(\overrightarrow{0}, \infty U_{1}\right),\left(\overrightarrow{0}, \infty U_{2}\right)\right\}=-\alpha_{g}\left\|U_{1}-U_{2}\right\|^{2}
$$

Example 4.2.3 Consider $\mathbb{H}=\ell^{2}$ and let $\zeta=\left(\zeta_{1}, \zeta_{2}, \zeta_{3} \cdots \zeta_{n}, \cdots\right)$, $\hat{\zeta}=\left(\hat{\zeta}_{1}, \hat{\zeta}_{2}, \hat{\zeta}_{3}, \cdots \hat{\zeta}_{n} \cdots\right)$ in $\ell^{2}$ therefore

$$
\begin{aligned}
& U_{1}=\frac{\zeta}{\left[\sum_{j=1}^{\infty}\left|\zeta_{j}\right|^{2}\right]^{1 / 2}}=\left(\varepsilon_{1}, \varepsilon_{2}, \varepsilon_{3}, \cdots \varepsilon_{n}, \cdots\right) \\
& U_{2}=\frac{\hat{\zeta}}{\left[\sum_{j=1}^{\infty}\left|\hat{\zeta}_{j}\right|^{2}\right]^{1 / 2}}=\left(\hat{\varepsilon}_{1}, \hat{\varepsilon}_{2}, \hat{\varepsilon}_{3}, \cdots \hat{\varepsilon}_{n}, \cdots\right)
\end{aligned}
$$

where

$$
\varepsilon_{j}=\frac{\zeta_{j}}{\left[\sum_{j=1}^{\infty}\left|\hat{\zeta}_{j}\right|^{2}\right]^{1 / 2}} \quad \text { and } \quad \hat{\varepsilon}_{j}=\frac{\hat{\zeta}_{j}}{\left[\sum_{j=1}^{\infty}\left|\hat{\zeta}_{j}\right|^{2}\right]^{1 / 2}}
$$

Thus

$$
D_{g}^{2}\left\{\left(\overrightarrow{0}, \infty U_{1}\right),\left(\overrightarrow{0}, \infty U_{2}\right)\right\}=-\alpha_{g}\left[\sum_{j=1}^{\infty}\left|\zeta-\hat{\zeta}_{j}\right|^{2}\right]^{1 / 2}
$$

Example 4.2.4 Consider $\mathbb{H}=L^{2}[0,1]$

$$
\left\langle f_{1}(s), f_{2}(s)\right\rangle=\int_{0}^{1} f_{1}(s) \overline{f_{2}(s)} d s
$$

where $f_{1}(s)$ and $f_{2}(s) \in[0,1]$ then we have

$$
D_{g}^{2}\left\{\left(\overrightarrow{0}, \infty U_{1}\right),\left(\overrightarrow{0}, \infty U_{2}\right)\right\}=-\alpha_{g} \int_{0}^{1}\left(U_{1}-U_{2}\right)(s) \overline{\left(U_{1}-U_{2}\right)(s)} d s
$$


Example 4.2.5 Let $\mathcal{L}(\mathbb{H}, \mathbb{H})$ be the set of all a linear transformation $T: \mathbb{H} \longrightarrow \mathbb{H}$ and define the norm on $\mathcal{L}(\mathbb{H}, \mathbb{H})$ as $\|T\|^{2}=\langle T, T\rangle_{\mathcal{L}}=$ $\operatorname{Trace}\left(T^{*} T\right)$ and $\left\langle T_{1}, T_{2}\right\rangle_{\mathcal{L}}=\operatorname{Trace}\left(T_{2}^{*} T_{1}\right)$ this norm is called Frobenius norm it is easy to prove the axioms of an inner product

1.

$$
\begin{aligned}
\left\langle T_{1}+T_{2}, T_{3}\right\rangle_{\mathcal{L}} & =\operatorname{Trace}\left(T_{3}^{*}\left(T_{1}+T_{2}\right)\right)=\operatorname{Trace}\left(T_{3}^{*} T_{1}+T_{3}^{*} T_{2}\right) \\
& =\operatorname{Trace}\left(T_{3}^{*} T_{1}\right)+\operatorname{Trace}\left(T_{3}^{*} T_{1}\right)=\left\langle T_{1}, T_{3}\right\rangle_{\mathcal{L}}+\left\langle T_{2}, T_{3}\right\rangle_{\mathcal{L}}
\end{aligned}
$$

2. let $a \in \mathbb{C}$ than we have

$$
\left\langle a T_{1}, T_{2}\right\rangle_{\mathcal{L}}=\operatorname{Trace}\left(T_{2}^{*} a T_{1}\right)=\operatorname{aTrace}\left(T_{2}^{*} T_{1}\right)=a\left\langle T_{1}, T_{2}\right\rangle_{\mathcal{L}}
$$

3.

$$
\left\langle T_{1}, a T_{2}\right\rangle_{\mathcal{L}}=\overline{\left\langle a T_{2}, T_{1}\right\rangle_{\mathcal{L}}}=\bar{a} \overline{\left\langle T_{2}, T_{1}\right\rangle_{\mathcal{L}}} \overline{\bar{a}} \overline{\left\langle T_{1}, T_{2}\right\rangle_{\mathcal{L}}}=
$$

4.

$$
\left\langle T_{1}, T_{2}\right\rangle_{\mathcal{L}}=\operatorname{Trace}\left(T_{2}^{*} T_{1}\right)=\operatorname{Trace}\left(\left(T_{1}^{*} T_{2}\right)^{*}\right)=\left(\operatorname{Trace}\left(T_{1}^{*} T_{2}\right)\right)^{*}=\left\langle T_{2}, T_{1}\right\rangle_{\mathcal{L}}^{*}
$$

5. $\left\langle T_{1}, T_{2}\right\rangle_{\mathcal{L}}=\operatorname{Trace}\left(T_{2}^{*} T_{1}\right) \geq 0$, also $\left\langle T_{1}, T_{1}\right\rangle_{\mathcal{L}}=\operatorname{Trace}\left(T_{1}^{*} T_{1}\right)=0$ if and only if $T_{1}=0$.

The Ultra Extended Hilbert space of $\mathcal{L}(\mathbb{H}, \mathbb{H})$ is

$$
\mathcal{L}(\mathbb{H}, \mathbb{H}) \cup\left\{\infty U \mid \operatorname{Trace}\left(U^{*} U\right)=1\right\}
$$

Consider $\left(\overrightarrow{0}, \infty U_{1}\right)$ and $\left(\overrightarrow{0}, \infty U_{2}\right) \in \mathcal{L}(\mathbb{H}, \mathbb{H}) \cup\left\{\infty U \mid \operatorname{Trace}\left(U^{*} U\right)=1\right\}$ then

$$
\begin{aligned}
& D_{g}^{2}\left\{\left(\overrightarrow{0}, \infty U_{1}\right),\left(\overrightarrow{0}, \infty U_{2}\right)\right\} \\
& =-\alpha_{g}\left\langle U_{1}-U_{2}, U_{1}-U_{2}\right\rangle_{\mathcal{L}}=-\alpha_{g} \operatorname{Trace}\left(\left(U_{1}-U_{2}\right)^{*}\left(U_{1}-U_{2}\right)\right)
\end{aligned}
$$

\subsection{On A Surface of A Sphere}

The main idea here is to define a bijection map from a subset of $\mathbb{U E H}$ which is $\left\{\left(\overrightarrow{0}, q_{n}\right): q_{n} \in \mathbb{U} \mathbb{E} \mathbb{H}\right\}$ into the Bowl and then we define a metric on $\mathbb{U} \mathbb{E} \mathbb{H}$ as follows. Denote by $\mathcal{G}_{s}$ the mapping matches each point $\left(\overrightarrow{0}, q_{n}\right) \in \mathbb{U} \mathbb{E} \mathbb{H}$ with a point $Z$ on a surface of a sphere and denote by $D_{s}\{Z, \hat{Z}\}=\|Z-\hat{Z}\|_{H_{N}}$ the distance between $Z$ and $\hat{Z}$ in $\mathbb{U E H}$. 
Definition 4.3.1 The mapping $\mathcal{G}_{s}\left(\left(\overrightarrow{0}, q_{n}\right)\right)$ from $\left\{\left(\overrightarrow{0}, q_{n}\right): q_{n} \in \mathbb{U} \mathbb{E} \mathbb{H}\right\}$ into the sphere is defined by

$$
\mathcal{G}_{s}\left(\left(\overrightarrow{0}, q_{n}\right)\right)= \begin{cases}\left(c_{n} u, t_{s n} q_{n}\right)=\left(\left(1-t_{s n}\right) \gamma_{s} u, t_{s n} q_{n}\right) & \text { if } q_{n} \in \mathbb{H} \\ \left(\gamma_{s} u, \psi(\infty) U\right)=\left(\gamma_{s} u, \sqrt{-\alpha_{s}} U\right) & \text { if } q_{n}=\infty U\end{cases}
$$

Proposition 4.3.1 The mapping $\mathcal{G}_{s}$ from $\left\{\left(\overrightarrow{0}, q_{n}\right): q_{n} \in \mathbb{U} \mathbb{E} \mathbb{H}\right\}$ into the sphere is bijection.

Remark 1. If $\alpha_{s}<0$ then we have $\left\|t_{s} q\right\|=0$ if and only if $q=0$

2. If $\alpha_{s}=0$ then we have $\left\|t_{s} q\right\|=0$ if and only if $\|q\|=0$ or $\|q\|=\infty$

\subsubsection{The Sphere Metric Induced}

In this section, we develop a metric on the set $\mathbb{U E H}$ that is induced by the sphere boundization

Theorem 4.3.2 the distance between any two points in $\mathbb{U E H}$ defines a metric as follows

1. Let $(\overrightarrow{0}, q)$ and $(\overrightarrow{0}, \hat{q})$ such that $q, \hat{q} \in \mathbb{H}$ then

$$
\begin{aligned}
& D_{s}^{2}\{(\overrightarrow{0}, q),(\overrightarrow{0}, \hat{q})\} \\
& =\left(\hat{t}_{s}-t_{s}\right)^{2} \gamma_{s} \bar{\gamma}_{s}+\left(t_{s}\|q\|-\hat{t}_{s}\|\hat{q}\|\right)^{2}+\hat{t}_{s} t_{s}(2\|q\|\|\hat{q}\|-\langle\hat{q}, q\rangle-\langle q, \hat{q}\rangle)
\end{aligned}
$$

2. Let $(\overrightarrow{0}, q),\left(\overrightarrow{0}, \infty U_{2}\right)$ such that $q \in \mathbb{H}$ then for $\alpha_{s}<0$ we have

$$
\begin{aligned}
& D_{s}^{2}\{(\overrightarrow{0}, q),(\overrightarrow{0}, \infty U)\} \\
& =t_{s}^{2} \gamma_{s} \bar{\gamma}_{s}+\left(t_{s}\|q\|-\sqrt{-\alpha_{s}}\right)^{2}+t_{s} \sqrt{-\alpha_{s}}(2\|q\|-\langle U, q\rangle-\langle q, U\rangle)
\end{aligned}
$$

3. Let $\left(\overrightarrow{0}, \infty U_{1}\right),\left(\overrightarrow{0}, \infty U_{2}\right)$ and $\alpha_{s}<0$ then

$$
D_{s}^{2}\left\{\left(\overrightarrow{0}, \infty U_{1}\right),\left(\overrightarrow{0}, \infty U_{2}\right)\right\}=-\alpha_{s}\left\|U_{1}-U_{2}\right\|^{2}
$$

where $U_{1}$ is a unit vector of $q$ and $U_{2}$ is a unit vector of $\hat{q}$ 
Moreover, the metric is continuous in $\mathbb{U} \mathbb{E} \mathbb{H}$ as follows.

$$
D_{s}^{2}\left\{(\overrightarrow{0}, q),\left(\overrightarrow{0}, \infty U_{2}\right)\right\}=\lim _{\left\|\hat{q}_{n}\right\| \longrightarrow \infty} D_{s}^{2}\left\{(\overrightarrow{0}, q),\left(\overrightarrow{0}, \hat{q}_{n}\right)\right\}
$$

and

$$
D_{s}^{2}\left\{\left(\overrightarrow{0}, \infty U_{1}\right),\left(\overrightarrow{0}, \infty U_{2}\right)\right\}=\lim _{\substack{\left\|q_{n}\right\| \longrightarrow \infty \\\left\|\hat{q}_{n}\right\| \longrightarrow \infty}} D_{s}^{2}\left\{\left(\overrightarrow{0}, q_{n}\right),\left(\overrightarrow{0}, \hat{q}_{n}\right)\right\}
$$

Proof To reach our goal, first we show that the following expression $\hat{t}_{s}-t_{s}$ has $\|q\|-\|\hat{q}\|$ as a common factor, after that it should be fairy obvious that the three axioms for a metric are satisfied in this case.

$$
\begin{aligned}
\hat{t}_{s}-t_{s} & =\frac{-\frac{\mathcal{A}}{2}+\sqrt{\hat{\hat{F}}}}{\|\hat{q}\|^{2}+\gamma_{s} \bar{\gamma}_{s}}-\frac{-\frac{\mathcal{A}}{2}+\sqrt{F}}{\|q\|^{2}+\gamma_{s} \bar{\gamma}_{s}} \\
& =\frac{\left(\|q\|^{2}+\gamma_{s} \bar{\gamma}_{s}\right)\left(-\frac{\mathcal{A}}{2}+\sqrt{\hat{F}}\right)-\left(\|\hat{q}\|^{2}+\gamma_{s} \bar{\gamma}_{s}\right)\left(-\frac{\mathcal{A}}{2}+\sqrt{F}\right)}{\left(\|\hat{q}\|^{2}+\gamma_{s} \bar{\gamma}_{s}\right)\left(\|q\|^{2}+\gamma_{s} \bar{\gamma}_{s}\right)}
\end{aligned}
$$

Put $N$ for the numerator of an equation (4.3.6) so that

$$
\begin{aligned}
& N=\|q\|^{2}\left(-\frac{\mathcal{A}}{2}\right)+\|q\|^{2} \sqrt{\hat{F}}+\gamma_{s} \bar{\gamma}_{s}\left(-\frac{\mathcal{A}}{2}\right)+\gamma_{s} \bar{\gamma}_{s} \sqrt{\hat{F}} \\
& -\|\hat{q}\|^{2}\left(-\frac{\mathcal{A}}{2}\right)-\|\hat{q}\|^{2} \sqrt{F}-\gamma_{s} \bar{\gamma}_{s}\left(-\frac{\mathcal{A}}{2}\right)-\gamma_{s} \bar{\gamma}_{s} \sqrt{F} \\
& =\|q\|^{2}\left(-\frac{\mathcal{A}}{2}\right)+\|q\|^{2} \sqrt{\hat{F}}+\gamma_{s} \bar{\gamma}_{s} \sqrt{\hat{F}}-\|\hat{q}\|^{2}\left(-\frac{\mathcal{A}}{2}\right)-\|\hat{q}\|^{2} \sqrt{F}-\gamma_{s} \bar{\gamma}_{s} \sqrt{F}
\end{aligned}
$$

add and subtract this value $\|q\|^{2} \sqrt{F}$ to $N$ we obtain

$$
\begin{aligned}
& N=\|q\|^{2}\left(-\frac{\mathcal{A}}{2}\right)+\|q\|^{2} \sqrt{\hat{F}}+\gamma_{s} \bar{\gamma}_{s} \sqrt{\hat{F}}+\|q\|^{2} \sqrt{F}-\|q\|^{2} \sqrt{F} \\
& -\|\hat{q}\|^{2}\left(-\frac{\mathcal{A}}{2}\right)-\|\hat{q}\|^{2} \sqrt{F}-\gamma_{s} \bar{\gamma}_{s} \sqrt{F} \\
& =\left(\|q\|^{2}-\|\hat{q}\|^{2}\right)\left(-\frac{\mathcal{A}}{2}\right)+\|q\|^{2}(\sqrt{\hat{F}}-\sqrt{F}) \\
& +\left(\|q\|^{2}-\|\hat{q}\|^{2}\right) \sqrt{F}+\gamma_{s} \bar{\gamma}_{s}(\sqrt{\hat{F}}-\sqrt{F}) \\
& =\left(\|q\|^{2}-\|\hat{q}\|^{2}\right)\left(-\frac{\mathcal{A}}{2}+\sqrt{F}\right)+\left(\|q\|^{2}+\gamma_{s} \bar{\gamma}_{s}\right)(\sqrt{\hat{F}}-\sqrt{F})
\end{aligned}
$$


thus

$$
\hat{t}_{s}-t_{s}=\frac{\left(\|q\|^{2}-\|\hat{q}\|^{2}\right)\left(-\frac{\mathcal{A}}{2}+\sqrt{F}\right)+\left(\|q\|^{2}+\gamma_{s} \bar{\gamma}_{s}\right)(\sqrt{\hat{F}}-\sqrt{F})}{\left(\|\hat{q}\|^{2}+\gamma_{s} \bar{\gamma}_{s}\right)\left(\|q\|^{2}+\gamma_{s} \bar{\gamma}_{s}\right)}
$$

Some simplification is needed, we use a simple trick worth remembering, we multiply the entire expression of $\hat{t}_{s}-t_{s}$ by this value $\frac{\sqrt{\hat{F}}+\sqrt{F}}{\sqrt{\hat{F}}+\sqrt{F}}$ which is convenient to simplify the expression to the following equation

$$
\begin{aligned}
& \hat{t}_{s}-t_{s} \\
& =\frac{\left(\|q\|^{2}-\|\hat{q}\|^{2}\right)\left(-\frac{\mathcal{A}}{2}+\sqrt{F}\right)(\sqrt{\hat{F}}+\sqrt{F})+\left(\|q\|^{2}+\gamma_{s} \bar{\gamma}_{s}\right)(\hat{F}-F)}{\left(\|\hat{q}\|^{2}+\gamma_{s} \bar{\gamma}_{s}\right)\left(\|q\|^{2}+\gamma_{s} \bar{\gamma}_{s}\right)(\sqrt{\hat{F}}+\sqrt{F})}
\end{aligned}
$$

we proceed to find a form $\hat{F}-F$ as follows

$$
\begin{aligned}
\hat{F}-F & =-\left(\|\hat{q}\|^{2}+\gamma_{s} \bar{\gamma}_{s}\right) \alpha_{s}+\left(\|q\|^{2}+\gamma_{s} \bar{\gamma}_{s}\right) \alpha_{s} \\
& =-\|\hat{q}\|^{2} \alpha_{s}-\gamma_{s} \bar{\gamma}_{s} \alpha_{s}+\|q\|^{2} \alpha_{s}+\gamma_{s} \bar{\gamma}_{s} \alpha_{s}=-\|\hat{q}\|^{2} \alpha_{s}+\|q\|^{2} \alpha_{s} \\
& =-\alpha_{s}\left(\|\hat{q}\|^{2}-\|q\|^{2}\right)=\alpha_{s}\left(\|q\|^{2}-\|\hat{q}\|^{2}\right)
\end{aligned}
$$

from the equation (4.3.8) we obtain

$$
\hat{t}_{s}-t_{s}=\frac{\left(\|q\|^{2}-\|\hat{q}\|^{2}\right)\left[\left(-\frac{\mathcal{A}}{2}+\sqrt{F}\right)(\sqrt{\hat{F}}+\sqrt{F})-\alpha_{s}\left(\|q\|^{2}+\gamma_{s} \bar{\gamma}_{s}\right)\right]}{\left(\|\hat{q}\|^{2}+\gamma_{s} \bar{\gamma}_{s}\right)\left(\|q\|^{2}+\gamma_{s} \bar{\gamma}_{s}\right)(\sqrt{\hat{F}}+\sqrt{F})}
$$

The first two conditions in the definition of a metric are obviously satisfied, indeed for positivity $D_{s}$ is nonnegative number $D_{s}\{(\overrightarrow{0}, q),(\overrightarrow{0}, \hat{q})\} \geq 0$ and by (4.3.9) $D_{s}$ only be equal to zero if and only if when $q=\hat{q}$ or when $\|q\|=\|\hat{q}\|$.

$$
D_{s}\{(\overrightarrow{0}, q),(\overrightarrow{0}, \hat{q})\}=0 \text { if and only if } q=\hat{q} \text { or }\|q\|=\|\hat{q}\| \text {. }
$$

And for symmetry we have

$$
D_{s}\{(\overrightarrow{0}, q),(\overrightarrow{0}, \hat{q})\}=\|(\overrightarrow{0}, q)-(\overrightarrow{0}, \hat{q})\|_{H_{N}}=\|(\overrightarrow{0}, \hat{q})-(\overrightarrow{0}, q)\|_{H_{N}}=D_{s}\{(\overrightarrow{0}, \hat{q}),(\overrightarrow{0}, q)\}
$$


To prove the triangle inequality, observe for any third point in $U E H(\overrightarrow{0}, q)$, $(\overrightarrow{0}, \hat{q})$ and $(\overrightarrow{0}, \tilde{q})$ we have

$$
\begin{aligned}
D_{s}\{(\overrightarrow{0}, q),(\overrightarrow{0}, \hat{q})\} & =\|(\overrightarrow{0}, q)-(\overrightarrow{0}, \hat{q})\|_{H_{N}} \\
& =\|(\overrightarrow{0}, q)-(\overrightarrow{0}, \tilde{q})+(\overrightarrow{0}, \tilde{q})-(\overrightarrow{0}, \hat{q})\|_{H_{N}} \\
& \leq\|(\overrightarrow{0}, q)-(\overrightarrow{0}, \tilde{q})\|_{H_{N}}+\|(\overrightarrow{0}, \tilde{q})-(\overrightarrow{0}, \hat{q})\|_{H_{N}} \\
& \leq D_{s}\{(\overrightarrow{0}, q),(\overrightarrow{0}, \tilde{q})\}+D_{s}\{(\overrightarrow{0}, \tilde{q}),(\overrightarrow{0}, \hat{q})\}
\end{aligned}
$$

Moreover,

$$
D_{s}^{2}\{(\overrightarrow{0}, q),(\overrightarrow{0}, \hat{q})\}=\left(\hat{t}_{s}-t_{s}\right)^{2} \gamma_{s} \bar{\gamma}_{s}+\left\langle t_{s} q-\hat{t}_{s} \hat{q}, t_{s} q-\hat{t}_{s} \hat{q}\right\rangle
$$

by the proposition (3.5.3) we have $\hat{t}_{s n} \hat{q}_{n} \sim \sqrt{-\alpha_{s}} U_{2}$ as $n \longrightarrow \infty$ and by the definition 4.3.1 we have $\hat{t}_{s} \hat{q}=\sqrt{-\alpha_{s}} U_{2}$ and as we know that when $\|\hat{q}\| \longrightarrow \infty$ then $\hat{t}_{s} \longrightarrow 0$ thus the equation (4.3.10) becomes

$$
D_{s}^{2}\left\{(\overrightarrow{0}, q),\left(\overrightarrow{0}, \infty U_{2}\right)\right\}=t_{s}^{2} \gamma_{s} \bar{\gamma}_{s}+\left\langle t_{s} q-\sqrt{-\alpha_{s}} U_{2}, t_{s} q-\sqrt{-\alpha_{s}} U_{2}\right\rangle
$$

therefore we get the equation (4.3.2). And when $\|q\| \longrightarrow \infty$ and $\|\hat{q}\| \longrightarrow \infty$ in the equation (4.3.1) we will get the equation (4.3.3) as follows. By propositions 3.5.3 and 3.5.4 we have $t_{s n} q_{n} \sim \sqrt{-\alpha_{s}} U_{1}$ and $\hat{t}_{s n} \hat{q}_{n} \sim \sqrt{-\alpha_{s}} U_{2}$ as $n \longrightarrow$ $\infty$ and by the definition 4.3 .1 we have $t_{s} q=\sqrt{-\alpha_{s}} U_{1}$ and $\hat{t}_{s} \hat{q}=\sqrt{-\alpha_{s}} U_{2}$. Also as we know when $\|q\| \longrightarrow \infty$ then $t_{s} \longrightarrow 0$ and when $\|\hat{q}\| \longrightarrow \infty$ then $\hat{t}_{s} \longrightarrow 0$ therefore the equation (4.3.10) becomes

$$
D_{s}^{2}\left\{\left(\overrightarrow{0}, \infty U_{2}\right),\left(\overrightarrow{0}, \infty U_{2}\right)\right\}=\left\langle\sqrt{-\alpha_{s}} U_{1}-\sqrt{-\alpha_{s}} U_{2}, \sqrt{-\alpha_{s}} U_{1}-\sqrt{-\alpha_{s}} U_{2}\right\rangle
$$

therefore we get (4.3.3)

\subsection{On A Surface of A Paraboloid}

In this section, we introduce the second metric by considering a surface as a paraboloid and we elaborate on some necessary language as we did in the previous section as follows. Denoted by $\mathcal{G}_{p}$ the mapping matches each point $\left(\overrightarrow{0}, q_{n}\right) \in \mathbb{U} \mathbb{E} \mathbb{H}$ with a point $Z$ on a surface of a paraboloid and denoted by $D_{p}\{Z, \hat{Z}\}=\|Z-\hat{Z}\|_{H_{N}}$ the distance between $Z$ and $\hat{Z}$ in $\mathbb{H}_{N}$. 
Definition 4.4.1 The mapping $\mathcal{G}_{p}\left(\left(\overrightarrow{0}, q_{n}\right)\right)$ from $\left\{\left(\overrightarrow{0}, q_{n}\right): q_{n} \in E U H\right\}$ into the paraboloid is defined by

$$
\mathcal{G}_{p}\left(\left(0, q_{n}\right)\right)= \begin{cases}\left(c_{n} u, t_{p n} q_{n}\right)=\left(\left(1-t_{p n}\right) \gamma_{p} u, t_{p n} q_{n}\right) & \text { if } q_{n} \in \mathbb{H} \\ \left(\gamma_{p} u, \psi(\infty) U\right)=\left(\gamma_{p} u, \sqrt{-\alpha_{p}} U\right) & \text { if } q_{n}=\infty U\end{cases}
$$

Proposition 4.4.1 The mapping $\mathcal{G}_{p}$ from $\left\{\left(0, q_{n}\right): q_{n} \in \mathbb{U} \mathbb{E} \mathbb{H}\right\}$ into the parabolic is bijection.

Remark $\left\|t_{p} q\right\|=0$ if and only if $\|q\|=0$

\subsubsection{The Paraboloid Metric Induced}

In this section, we develop a metric on the set $\mathbb{U} \mathbb{E} \mathbb{H}$ that is induced by the paraboloid boundization

Theorem 4.4.2 the distance between any two points in $\mathbb{U} \mathbb{E} \mathbb{H}$ defines a metric as follows

1. Let $(\overrightarrow{0}, q)$ and $(\overrightarrow{0}, \hat{q})$ such that $q, \hat{q} \in \mathbb{H}$ then

$$
\begin{aligned}
& D_{p}^{2}\{(\overrightarrow{0}, q),(\overrightarrow{0}, \hat{q})\} \\
& =\left(\hat{t}_{p}-t_{p}\right)^{2} \gamma_{p} \bar{\gamma}_{p}+\left(t_{p}\|q\|-\hat{t}_{p}\|\hat{q}\|\right)^{2}+\hat{t}_{p} t_{p}(2\|q\|\|\hat{q}\|-\langle\hat{q}, q\rangle-\langle q, \hat{q}\rangle)
\end{aligned}
$$

2. Let $(\overrightarrow{0}, q),\left(\overrightarrow{0}, \infty U_{2}\right)$ such that $q \in \mathbb{H}$ then we have

$$
\begin{aligned}
& D_{p}^{2}\{(\overrightarrow{0}, q),(\overrightarrow{0}, \infty U)\} \\
& =t_{p}^{2} \gamma_{p} \bar{\gamma}_{p}+\left(t_{p}\|q\|-\sqrt{-\alpha_{p}}\right)^{2}+\sqrt{-\alpha_{p}}(2\|q\|-\langle U, q\rangle-\langle q, U\rangle)
\end{aligned}
$$

3. Let $\left(\overrightarrow{0}, \infty U_{1}\right),\left(\overrightarrow{0}, \infty U_{2}\right)$ then

$$
D_{p}^{2}\left\{\left(\overrightarrow{0}, \infty U_{1}\right),\left(\overrightarrow{0}, \infty U_{2}\right)\right\}=-\alpha_{p}\left\|U_{1}-U_{2}\right\|^{2}
$$

where $U_{1}$ is a unit vector of $q$ and $U_{2}$ is a unit vector of $\hat{q}$ 
Moreover, the metric is continuous in $\mathbb{U E H}$ as follows.

$$
D_{p}^{2}\left\{(\overrightarrow{0}, q),\left(\overrightarrow{0}, \infty U_{2}\right)\right\}=\lim _{\left\|\hat{q}_{n}\right\| \longrightarrow \infty} D_{p}^{2}\left\{(\overrightarrow{0}, q),\left(\overrightarrow{0}, \hat{q}_{n}\right)\right\}
$$

and

$$
D_{p}^{2}\left\{\left(\overrightarrow{0}, \infty U_{1}\right),\left(\overrightarrow{0}, \infty U_{2}\right)\right\}=\lim _{\substack{\left\|q_{n}\right\| \longrightarrow \infty \\\left\|\hat{q}_{n}\right\| \longrightarrow \infty}} D_{p}^{2}\left\{\left(\overrightarrow{0}, q_{n}\right),\left(\overrightarrow{0}, \hat{q}_{n}\right)\right\}
$$

Proof As we did in the previous subsection, first we show that the following expression $\hat{t}_{p}-t_{p}$ has $\|q\|-\|\hat{q}\|$ as a common factor, after that it should be fairly obvious that the three axioms for a metric are satisfied in this case.

$$
\begin{aligned}
\hat{t}_{p}-t_{p}= & \frac{-2 \alpha_{p}}{\mathcal{B}+\mathcal{B} \sqrt{\hat{G}}}-\frac{-2 \alpha_{p}}{\mathcal{B}+\mathcal{B} \sqrt{G}} \\
= & \frac{-2 \alpha_{p}((\mathcal{B}+\mathcal{B} \sqrt{G})-(\mathcal{B}+\mathcal{B} \sqrt{\hat{G}}))}{(\mathcal{B}+\mathcal{B} \sqrt{\hat{G}})(\mathcal{B}+\mathcal{B} \sqrt{G})} \\
= & \frac{-2 \alpha_{p}(\mathcal{B} \sqrt{G}-\mathcal{B} \sqrt{\hat{G}})}{(\mathcal{B}+\mathcal{B} \sqrt{\hat{G}})(\mathcal{B}+\mathcal{B} \sqrt{G})}=\frac{-2 \alpha_{p}(\sqrt{G}-\sqrt{\hat{G}})}{\mathcal{B}(1+\sqrt{\hat{G}})(1+\sqrt{G})} \\
= & \frac{-2 \alpha_{p}(\sqrt{G}-\sqrt{\hat{G}})(\sqrt{G}+\sqrt{\hat{G}})}{\mathcal{B}(1+\sqrt{\hat{G}})(1+\sqrt{G})(\sqrt{G}+\sqrt{\hat{G}})} \\
= & \frac{-2 \alpha_{p}\left(1-\frac{4\|q\|^{2} \alpha_{p}}{\mathcal{B}^{2}}-1+\frac{4\|\hat{\|}\|^{2} \alpha_{p}}{\mathcal{B}^{2}}\right)}{\mathcal{B}(1+\sqrt{\hat{G}})(1+\sqrt{G})(\sqrt{G}+\sqrt{\hat{G}})} \\
= & \frac{-2 \alpha_{p}\left(\frac{4\|\hat{\|}\|^{2} \alpha_{p}}{\mathcal{B}^{2}}-\frac{4\|q\|^{2} \alpha_{p}}{\mathcal{B}^{2}}\right)}{\mathcal{B}(1+\sqrt{\hat{G}})(1+\sqrt{G})(\sqrt{G}+\sqrt{\hat{G}})}
\end{aligned}
$$

therefore

$$
\hat{t}_{p}-t_{p}=\frac{8 \alpha_{p}^{2}\left(\|q\|^{2}-\|\hat{q}\|^{2}\right)}{\mathcal{B}^{3}(1+\sqrt{\hat{G}})(1+\sqrt{G})(\sqrt{G}+\sqrt{\hat{G}})}
$$

The first two conditions in the definition of a metric are obviously satisfied, namely for positivity $D_{p}$ is nonnegative number $D_{p}\{(\overrightarrow{0}, q),(\overrightarrow{0}, \hat{q})\} \geq 0$ and by 
(4.4.6) $D_{p}$ only be equal to zero if and only if when $q=\hat{q}$ or when $\|q\|=\|\hat{q}\|$.

$$
D_{p}\{(\overrightarrow{0}, q),(\overrightarrow{0}, \hat{q})\}=0 \text { if and only if } q=\hat{q} \text { or }\|q\|=\|\hat{q}\| .
$$

And for symmetry we have

$D_{p}\{(\overrightarrow{0}, q),(\overrightarrow{0}, \hat{q})\}=\|(\overrightarrow{0}, q)-(0, \hat{q})\|_{H_{N}}=\|(\overrightarrow{0}, \hat{q})-(\overrightarrow{0}, q)\|_{H_{N}}=D_{p}\{(\overrightarrow{0}, \hat{q}),(\overrightarrow{0}, q)\}$

To prove the triangle inequality, observe for any third point in $\mathbb{U E H}(\overrightarrow{0}, q)$, $(\overrightarrow{0}, \hat{q})$ and $(\overrightarrow{0}, \tilde{q})$ we have

$$
\begin{aligned}
D_{p}\{(\overrightarrow{0}, q),(\overrightarrow{0}, \hat{q})\} & =\|(\overrightarrow{0}, q)-(\overrightarrow{0}, \hat{q})\|_{H_{N}} \\
& =\|(\overrightarrow{0}, q)-(\overrightarrow{0}, \tilde{q})+(\overrightarrow{0}, \tilde{q})-(\overrightarrow{0}, \hat{q})\|_{H_{N}} \\
& \leq\|(\overrightarrow{0}, q)-(\overrightarrow{0}, \tilde{q})\|_{H_{N}}+\|(\overrightarrow{0}, \tilde{q})-(0, \hat{q})\|_{H_{N}} \\
& \leq D_{p}\{(\overrightarrow{0}, q),(\overrightarrow{0}, \tilde{q})\}+D_{p}\{(\overrightarrow{0}, \tilde{q}),(0, \hat{q})\}
\end{aligned}
$$

Moreover,

$$
D_{p}^{2}\{(\overrightarrow{0}, q),(\overrightarrow{0}, \hat{q})\}=\left(\hat{t}_{P}-t_{p}\right)^{2} \gamma_{p} \bar{\gamma}_{p}+\left\langle t_{p} q-\hat{t}_{p} \hat{q}, t_{p} q-\hat{t}_{p} \hat{q}\right\rangle
$$

by the proposition (3.6.2) we have $\hat{t}_{p n} \hat{q}_{n} \sim \sqrt{-\alpha_{p}} U_{2}$ as $n \longrightarrow \infty$ and by the definition 4.4.1 we have $\hat{t}_{p} \hat{q}=\sqrt{-\alpha_{p}} U_{2}$ and as we know that when $\|\hat{q}\| \longrightarrow \infty$ then $\hat{t}_{p} \longrightarrow 0$ thus the equation (4.4.7) becomes

$$
D_{p}^{2}\left\{(\overrightarrow{0}, q),\left(\overrightarrow{0}, \infty U_{2}\right)\right\}=t_{p}^{2} \gamma_{p} \bar{\gamma}_{p}+\left\langle t_{p} q-\sqrt{-\alpha_{p}} U_{2}, t_{p} q-\sqrt{-\alpha_{p}} U_{2}\right\rangle
$$

therefore we get the equation (4.4.2). And when $\|q\| \longrightarrow \infty$ and $\|\hat{q}\| \longrightarrow \infty$ in the equation (4.4.1) we will get the equation (4.4.3) as follows. By propositions 3.6.2 and 3.6.3 we have $t_{p n} q_{n} \sim \sqrt{-\alpha_{p}} U_{1}$ and $\hat{t}_{p n} \hat{q}_{n} \sim \sqrt{-\alpha_{p}} U_{2}$ as $n \longrightarrow$ $\infty$ and by the definition 4.4.1 we have $t_{p} q=\sqrt{-\alpha_{p}} U_{1}$ and $\hat{t_{p}} \hat{q}=\sqrt{-\alpha_{p}} U_{2}$ also as we know when $\|q\| \longrightarrow \infty$ then $t_{p} \longrightarrow 0$ and when $\|\hat{q}\| \longrightarrow \infty$ then $\hat{t}_{p} \longrightarrow 0$ therefore the equation (4.4.7) becomes

$$
D_{p}^{2}\left\{\left(\overrightarrow{0}, \infty U_{2}\right),\left(\overrightarrow{0}, \infty U_{2}\right)\right\}=\left\langle\sqrt{-\alpha_{p}} U_{1}-\sqrt{-\alpha_{p}} U_{2}, \sqrt{-\alpha_{p}} U_{1}-\sqrt{-\alpha_{p}} U_{2}\right\rangle
$$

therefore we get (4.4.3) 


\subsection{On A Surface of A Cone}

The main idea here it is similar to what we did in the previous section. We consider that a surface is a cone and we elaborate on some necessary language as well. Denoted by $\mathcal{G}_{c}$ the mapping matches each point $\left(\overrightarrow{0}, q_{n}\right) \in \mathbb{U} \mathbb{E} \mathbb{H}$ with a point $Z$ on a surface of a cone and denoted by $D_{c}\{Z, \hat{Z}\}=\|Z-\hat{Z}\|_{H_{N}}$ the distance between $Z$ and $\hat{Z}$ in $\mathbb{H}_{N}$.

Definition 4.5.1 The mapping $\mathcal{G}_{c}\left(\left(\overrightarrow{0}, q_{n}\right)\right)$ from $\left\{\left(\overrightarrow{0}, q_{n}\right): q_{n} \in \mathbb{U} \mathbb{E} \mathbb{H}\right\}$ into the cone is defined by

$$
\mathcal{G}_{c}\left(\left(\overrightarrow{0}, q_{n}\right)\right)= \begin{cases}\left(c_{n} u, t_{c n} q_{n}\right)=\left(\left(1-t_{c n}\right) \gamma_{s} u, t_{c n} q_{n}\right) & \text { if } q_{n} \in \mathbb{H} \\ \left(\gamma_{c} u, \psi(\infty) U\right)=\left(\gamma_{c} u, \alpha_{c} U\right) & \text { if } q_{n}=\infty U\end{cases}
$$

Proposition 4.5.1 The mapping $\mathcal{G}_{c}$ from $\left\{\left(\overrightarrow{0}, q_{n}\right): q_{n} \in E U H\right\}$ into the cone is bijection.

Remark $\left\|t_{c} q\right\|=0$ if and only if $\|q\|=0$

\subsubsection{The Cone Metric Induced}

In this section, we develop a metric on the set $\mathbb{U E H}$ that is induced by the cone boundization

Theorem 4.5.2 the distance between any two points in $\mathbb{U} \mathbb{E} \mathbb{I}$ defines a metric as follows

1. Let $(\overrightarrow{0}, q)$ and $(\overrightarrow{0}, \hat{q})$ such that $q, \hat{q} \in \mathbb{H}$ then

$$
\begin{aligned}
& D_{c}^{2}\{(\overrightarrow{0}, q),(\overrightarrow{0}, \hat{q})\} \\
& =\left(\hat{t}_{c}-t_{c}\right)^{2} \gamma_{c} \bar{\gamma}_{c}+\left(t_{c}\|q\|-\hat{t}_{c}\|\hat{q}\|\right)^{2}+\hat{t}_{c} t_{c}(2\|q\|\|\hat{q}\|-\langle\hat{q}, q\rangle-\langle q, \hat{q}\rangle)
\end{aligned}
$$

2. Let $(\overrightarrow{0}, q),\left(\overrightarrow{0}, \infty U_{2}\right)$ such that $q \in \mathbb{H}$ then we have

$$
D_{c}^{2}\{(\overrightarrow{0}, q),(\overrightarrow{0}, \infty U)\}=t_{c}^{2} \gamma_{c} \bar{\gamma}_{c}+\left(t_{c}\|q\|-\alpha_{c}\right)^{2}+\alpha_{c} t_{c}(2\|q\|-\langle U, q\rangle-\langle q, U\rangle)
$$


3. Let $\left(\overrightarrow{0}, \infty U_{1}\right),\left(\overrightarrow{0}, \infty U_{2}\right)$ then

$$
D_{c}^{2}\left\{\left(\overrightarrow{0}, \infty U_{1}\right),\left(\overrightarrow{0}, \infty U_{2}\right)\right\}=\alpha_{c}^{2}\left\|U_{1}-U_{2}\right\|^{2}
$$

where $U_{1}$ is a unit vector of $q$ and $U_{2}$ is a unit vector of $\hat{q}$

Moreover, the metric is continuous in $\mathbb{U} \mathbb{E} \mathbb{H}$ as follows.

$$
D_{c}^{2}\left\{(\overrightarrow{0}, q),\left(\overrightarrow{0}, \infty U_{2}\right)\right\}=\lim _{\left\|\hat{q}_{n}\right\| \longrightarrow \infty} D_{c}^{2}\left\{(\overrightarrow{0}, q),\left(\overrightarrow{0}, \hat{q}_{n}\right)\right\}
$$

and

$$
D_{c}^{2}\left\{\left(\overrightarrow{0}, \infty U_{1}\right),\left(\overrightarrow{0}, \infty U_{2}\right)\right\}=\lim _{\substack{\left\|q_{n}\right\| \longrightarrow \infty \\\left\|\hat{q}_{n}\right\| \longrightarrow \infty}} D_{c}^{2}\left\{\left(\overrightarrow{0}, q_{n}\right),\left(\overrightarrow{0}, \hat{q}_{n}\right)\right\}
$$

Proof To reach our goal, first we show that the following expression $\hat{t}_{c}-t_{c}$ has $\|q\|-\|\hat{q}\|$ as a common factor, after that it should be obvious that the three axioms for a metric are satisfied in this case. Since

$$
\hat{t}_{c}=\frac{\alpha_{c}}{\|\hat{q}\|+\frac{\left|\gamma_{c}\right|}{m}}, \quad t_{c}=\frac{\alpha_{c}}{\|q\|+\frac{\left|\gamma_{c}\right|}{m}},
$$

SO

$$
\hat{t}_{c}-t_{c}=\frac{\alpha_{c}}{\|\hat{q}\|+\frac{\left|\gamma_{c}\right|}{m}}-\frac{\alpha_{c}}{\|q\|+\frac{\left|\gamma_{c}\right|}{m}}=\frac{\alpha_{c}(\|q\|-\|\hat{q}\|)}{\left(\|\hat{q}\|+\frac{\left|\gamma_{c}\right|}{m}\right)\left(\|q\|+\frac{\left|\gamma_{c}\right|}{m}\right)}
$$

The first two conditions in the definition of a metric are obviously satisfied, namely for positivity $D_{c}$ is nonnegative number $D_{c}\{(\overrightarrow{0}, q),(\overrightarrow{0}, \hat{q})\} \geq 0$ and by (4.5.6) $D_{c}$ only be equal to zero if and only if when $q=\hat{q}$ or when $\|q\|=\|\hat{q}\|$.

$$
D_{c}\{(\overrightarrow{0}, q),(\overrightarrow{0}, \hat{q})\}=0 \text { if and only if } q=\hat{q} \text { or }\|q\|=\|\hat{q}\| .
$$

And for symmetry we have

$$
D_{c}\{(\overrightarrow{0}, q),(\overrightarrow{0}, \hat{q})\}=\|(\overrightarrow{0}, q)-(\overrightarrow{0}, \hat{q})\|_{H_{N}}=\|(\overrightarrow{0}, \hat{q})-(\overrightarrow{0}, q)\|_{H_{N}}=D_{c}\{(\overrightarrow{0}, \hat{q}),(\overrightarrow{0}, q)\}
$$

To prove the triangle inequality, observe for any third point in $U E H(\overrightarrow{0}, q)$, $(\overrightarrow{0}, \hat{q})$ and $(\overrightarrow{0}, \tilde{q})$ we have

$$
\begin{aligned}
D_{c}\{(\overrightarrow{0}, q),(\overrightarrow{0}, \hat{q})\} & =\|(\overrightarrow{0}, q)-(\overrightarrow{0}, \hat{q})\|_{H_{N}} \\
& =\|(\overrightarrow{0}, q)-(\overrightarrow{0}, \tilde{q})+(\overrightarrow{0}, \tilde{q})-(\overrightarrow{0}, \hat{q})\|_{H_{N}} \\
& \leq\|(\overrightarrow{0}, q)-(\overrightarrow{0}, \tilde{q})\|_{H_{N}}+\|(\overrightarrow{0}, \tilde{q})-(\overrightarrow{0}, \hat{q})\|_{H_{N}} \\
& \leq D_{c}\{(\overrightarrow{0}, q),(\overrightarrow{0}, \tilde{q})\}+D_{c}\{(\overrightarrow{0}, \tilde{q}),(\overrightarrow{0}, \hat{q})\}
\end{aligned}
$$


Moreover,

$$
D_{c}^{2}\{(\overrightarrow{0}, q),(\overrightarrow{0}, \hat{q})\}=\left(\hat{t}_{c}-t_{c}\right)^{2} \gamma_{c} \bar{\gamma}_{c}+\left\langle t_{c} q-\hat{t}_{c} \hat{q}, t_{c} q-\hat{t}_{c} \hat{q}\right\rangle
$$

by the proposition (3.7.2) we have $\hat{t}_{c n} \hat{q}_{n} \sim \alpha_{c} U_{2}$ as $n \longrightarrow \infty$ and by the definition 4.5.1 we have $\hat{t}_{c} \hat{q}=\alpha_{c} U_{2}$ and as we know that when $\|\hat{q}\| \longrightarrow \infty$ then $\hat{t}_{c} \longrightarrow 0$ thus the equation (4.5.7) becomes

$$
D_{c}^{2}\left\{(\overrightarrow{0}, q),\left(\overrightarrow{0}, \infty U_{2}\right)\right\}=t_{c}^{2} \gamma_{c} \bar{\gamma}_{c}+\left\langle t_{c} q-\left(\gamma_{c}-\theta_{c}\right) U_{2}, t_{c} q-\left(\gamma_{c}-\theta_{c}\right) U_{2}\right\rangle
$$

therefore we get the equation (4.5.2) . And when $\|q\| \longrightarrow \infty$ and $\|\hat{q}\| \longrightarrow$ $\infty$ in the equation (4.5.1) we will get the equation (4.5.3) as follows. By propositions 3.7.2 and 3.7.3 we have $t_{s n} q_{n} \sim \alpha_{c} U_{1}$ and $\hat{t}_{s n} \hat{q}_{n} \sim \alpha_{c} U_{2}$ as $n \longrightarrow$ $\infty$ and by the definition 4.5.1 we have $t_{c} q=\alpha_{c} U_{1}$ and $\hat{t}_{c} \hat{q}=\alpha_{c} U_{2}$ also as we know when $\|q\| \longrightarrow \infty$ then $t_{c} \longrightarrow 0$ and when $\|\hat{q}\| \longrightarrow \infty$ then $\hat{t}_{c} \longrightarrow 0$ therefore the equation (4.5.7) becomes

$$
D_{c}^{2}\left\{\left(\overrightarrow{0}, \infty U_{2}\right),\left(\overrightarrow{0}, \infty U_{2}\right)\right\}=\left\langle\alpha_{c} U_{1}-\alpha_{c} U_{2}, \alpha_{c} U_{1}-\alpha_{c} U_{2}\right\rangle
$$

therefore we get (4.5.3)

\subsection{On A Surface of A Hyperboloid}

In this section we consider a surface is a hyperboloid and we elaborate on some necessary language as we did in the previous section as follows. Denote by $\mathcal{G}_{h}$ the mapping matches each point $\left(\overrightarrow{0}, q_{n}\right) \in \mathbb{U} \mathbb{E} \mathbb{H}$ with a point $Z$ on a surface of a hyperboloid and denote by $D_{h}\{Z, \hat{Z}\}=\|Z-\hat{Z}\|_{H_{N}}$ the distance between $Z$ and $\hat{Z}$ in $\mathbb{H}_{N}$.

Definition 4.6.1 The mapping $\mathcal{G}_{h}\left(\left(\overrightarrow{0}, q_{n}\right)\right)$ from $\left\{\left(\overrightarrow{0}, q_{n}\right): q_{n} \in \mathbb{U} \mathbb{E} \mathbb{H}\right\}$ into the hyperboloid is defined by

$$
\mathcal{G}_{h}\left(\left(\overrightarrow{0}, q_{n}\right)\right)= \begin{cases}\left(c_{n} u, t_{h n} q_{n}\right)=\left(\left(1-t_{h n}\right) \gamma_{h} u, t_{h n} q_{n}\right) & \text { if } q_{n} \in \mathbb{H} \\ \left(\gamma_{h} u, \psi(\infty) U\right)=\left(\gamma_{h} u, \sqrt{-\alpha_{h}} U\right) & \text { if } q_{n}=\infty U\end{cases}
$$

Proposition 4.6.1 The mapping $\mathcal{G}_{h}$ from $\left\{\left(\overrightarrow{0}, q_{n}\right): q_{n} \in \mathbb{U} \mathbb{E} \mathbb{H}\right\}$ into the hyperboloid is bijection.

Remark $\left\|t_{h} q\right\|=0$ if and only if $\|q\|=0$ 


\subsubsection{The Hyperboloid Metric Induced}

In this section, we develop a metric on the set $\mathbb{U E H}$ that is induced by the hyperboloid boundization

Theorem 4.6.2 the distance between any two points in $\mathbb{U} \mathbb{E} \mathbb{H}$ defines a metric as follows

1. Let $(\overrightarrow{0}, q)$ and $(\overrightarrow{0}, \hat{q})$ such that $q, \hat{q} \in \mathbb{H}$ then

$$
\begin{aligned}
& D_{h}^{2}\{(\overrightarrow{0}, q),(\overrightarrow{0}, \hat{q})\} \\
& =\left(\hat{t}_{h}-t_{h}\right)^{2} \gamma_{h} \bar{\gamma}_{h}+\left(t_{h}\|q\|-\hat{t}_{h}\|\hat{q}\|\right)^{2}+\hat{t}_{h} t_{h}(2\|q\|\|\hat{q}\|-\langle\hat{q}, q\rangle-\langle q, \hat{q}\rangle)
\end{aligned}
$$

2. Let $(\overrightarrow{0}, q),\left(\overrightarrow{0}, \infty U_{2}\right)$ such that $q \in \mathbb{H}$ then we have

$$
\begin{aligned}
& D_{h}^{2}\{(\overrightarrow{0}, q),(\overrightarrow{0}, \infty U)\} \\
& =t_{h}^{2} \gamma_{h} \bar{\gamma}_{h}+\left(t_{h}\|q\|-\sqrt{-\alpha_{h}}\right)^{2}+\sqrt{-\alpha_{h}}(2\|q\|-\langle U, q\rangle-\langle q, U\rangle)
\end{aligned}
$$

3. Let $\left(\overrightarrow{0}, \infty U_{1}\right),\left(\overrightarrow{0}, \infty U_{2}\right)$ then

$$
D_{h}^{2}\left\{\left(\overrightarrow{0}, \infty U_{1}\right),\left(\overrightarrow{0}, \infty U_{2}\right)\right\}=-\alpha_{h}\left\|U_{1}-U_{2}\right\|^{2}
$$

where $U_{1}$ is a unit vector of $q$ and $U_{2}$ is a unit vector of $\hat{q}$

Moreover, the metric is continuous in $\mathbb{U} \mathbb{E} \mathbb{H}$ as follows.

$$
D_{h}^{2}\left\{(\overrightarrow{0}, q),\left(\overrightarrow{0}, \infty U_{2}\right)\right\}=\lim _{\left\|\hat{q}_{n}\right\| \longrightarrow \infty} D_{h}^{2}\left\{(\overrightarrow{0}, q),\left(\overrightarrow{0}, \hat{q}_{n}\right)\right\}
$$

and

$$
D_{h}^{2}\left\{\left(\overrightarrow{0}, \infty U_{1}\right),\left(\overrightarrow{0}, \infty U_{2}\right)\right\}=\lim _{\substack{\left\|q_{n}\right\| \longrightarrow \infty \\\left\|\hat{q}_{n}\right\| \longrightarrow \infty}} D_{h}^{2}\left\{\left(\overrightarrow{0}, q_{n}\right),\left(\overrightarrow{0}, \hat{q}_{n}\right)\right\}
$$

Proof As we did in the previous subsection ,first we show that the following expression $\hat{t}_{h}-t_{h}$ has $\|q\|-\|\hat{q}\|$ as a common factor, after that it should be fairy obvious that the three axioms for a metric are satisfied in this case.

$$
\hat{t}_{h}-t_{h}=\frac{-\alpha_{h}}{\mathcal{C}+\sqrt{\hat{E}}}-\frac{-\alpha_{h}}{\mathcal{C}+\sqrt{E}}=\frac{-\alpha_{h}(\sqrt{E}-\sqrt{\hat{E}})}{(\mathcal{C}+\sqrt{\hat{E}})(\mathcal{C}+\sqrt{E})}
$$


We rewrite the fraction by rationalizing the numerator

$$
\hat{t}_{h}-t_{h}=\frac{-\alpha_{h}\left(\mathcal{C}^{2}-\left(\|q\|^{2}-\mathcal{C}\right) \alpha_{h}-\mathcal{C}^{2}+\left(\|\hat{q}\|^{2}-\mathcal{C}\right) \alpha_{h}\right)}{(\mathcal{C}+\sqrt{\hat{E}})(\mathcal{C}+\sqrt{E})(\sqrt{E}+\sqrt{\hat{E}})}
$$

therefore

$$
\hat{t}_{h}-t_{h}=\frac{\alpha_{h}^{2}\left(\|q\|^{2}-\|\hat{q}\|^{2}\right)}{(\mathcal{C}+\sqrt{\hat{E}})(\mathcal{C}+\sqrt{E})(\sqrt{E}+\sqrt{\hat{E}})}
$$

The first two conditions in the definition of a metric are obviously satisfied, namely for positivity $D_{h}$ is nonnegative number $D_{p}\{(\overrightarrow{0}, q),(\overrightarrow{0}, \hat{q})\} \geq 0$ and by (4.6.6) $D_{h}$ only be equal to zero if and only if when $q=\hat{q}$ or when $\|q\|=\|\hat{q}\|$.

$$
D_{h}\{(\overrightarrow{0}, q),(\overrightarrow{0}, \hat{q})\}=0 \text { if and only if } q=\hat{q} \text { or }\|q\|=\|\hat{q}\| \text {. }
$$

And for symmetry we have

$$
\begin{aligned}
D_{h}\{(\overrightarrow{0}, q),(\overrightarrow{0}, \hat{q})\} & =\|(\overrightarrow{0}, q)-(\overrightarrow{0}, \hat{q})\|_{H_{N}} \\
& =\|(\overrightarrow{0}, \hat{q})-(\overrightarrow{0}, q)\|_{H_{N}}=D_{h}\{(\overrightarrow{0}, \hat{q}),(\overrightarrow{0}, q)\}
\end{aligned}
$$

To prove the triangle inequality, observe for any third point in $U E H(\overrightarrow{0}, q)$, $(\overrightarrow{0}, \hat{q})$ and $(\overrightarrow{0}, \tilde{q})$ we have

$$
\begin{aligned}
D_{h}\{(\overrightarrow{0}, q),(\overrightarrow{0}, \hat{q})\} & =\|(\overrightarrow{0}, q)-(\overrightarrow{0}, \hat{q})\|_{H_{N}} \\
& =\|(\overrightarrow{0}, q)-(\overrightarrow{0}, \tilde{q})+(\overrightarrow{0}, \tilde{q})-(\overrightarrow{0}, \hat{q})\|_{H_{N}} \\
& \leq\|(\overrightarrow{0}, q)-(\overrightarrow{0}, \tilde{q})\|_{H_{N}}+\|(\overrightarrow{0}, \tilde{q})-(\overrightarrow{0}, \hat{q})\|_{H_{N}} \\
& \leq D_{h}\{(\overrightarrow{0}, q),(\overrightarrow{0}, \tilde{q})\}+D_{h}\{(\overrightarrow{0}, \tilde{q}),(\overrightarrow{0}, \hat{q})\}
\end{aligned}
$$

Moreover,

$$
D_{h}^{2}\{(\overrightarrow{0}, q),(\overrightarrow{0}, \hat{q})\}=\left(\hat{t}_{h}-t_{h}\right)^{2} \gamma_{h} \bar{\gamma}_{h}+\left\langle t_{h} q-\hat{t}_{h} \hat{q}, t_{h} q-\hat{t}_{h} \hat{q}\right\rangle
$$

by the proposition (3.8.4) we have $\hat{t}_{h n} \hat{q}_{n} \sim \sqrt{-\alpha_{h}} U_{2}$ as $n \longrightarrow \infty$ and by the definition 4.6.1 we have $\hat{t}_{h} \hat{q}=\sqrt{-\alpha_{h}} U_{2}$ and as we know that when $\|\hat{q}\| \longrightarrow \infty$ then $\hat{t}_{h} \longrightarrow 0$ thus the equation (4.6.7) becomes

$$
D_{h}^{2}\left\{(\overrightarrow{0}, q),\left(\overrightarrow{0}, \infty U_{2}\right)\right\}=t_{h}^{2} \gamma_{h} \bar{\gamma}_{h}+\left\langle t_{h} q+\sqrt{-\alpha_{h}} U_{2}, t_{h} q-\sqrt{-\alpha_{h}} U_{2}\right\rangle .
$$


Therefore, we get the equation (4.6.2) . And when $\|q\| \longrightarrow \infty$ and $\|\hat{q}\| \longrightarrow \infty$ in the equation (4.6.1) we will get the equation (4.6.3) as follows, by propositions 3.8.4 and 3.8.5 we have $t_{h n} q_{n} \sim \sqrt{-\alpha_{h}} U_{1}$ and $\hat{t}_{h n} \hat{q}_{n} \sim \sqrt{-\alpha_{h}} U_{2}$ as $n \longrightarrow$ $\infty$ and by the definition 4.6.1 we have $t_{h} q=\sqrt{-\alpha_{h}} U_{1}$ and $\hat{t}_{h} \hat{q}=\sqrt{-\alpha_{h}} U_{2}$ also as we know when $\|q\| \longrightarrow \infty$ then $t_{h} \longrightarrow 0$ and when $\|\hat{q}\| \longrightarrow \infty$ then $\hat{t}_{h} \longrightarrow 0$ therefore the equation (4.6.7) becomes

therefore we get (4.6.3)

\subsection{On a Surface of An Ellipsoid}

The main idea here is to define a bijection map from a subset of $\mathbb{U E \mathbb { H }}$ which is $\left\{\left(\overrightarrow{0}, q_{n}\right): q_{n} \in \mathbb{U} \mathbb{E} \mathbb{H}\right\}$ into the Bowl and then we define a metric on $\mathbb{U} \mathbb{E} \mathbb{H}$ as follows. Denote by $\mathcal{G}_{e}$ the mapping matches each point $\left(\overrightarrow{0}, q_{n}\right) \in \mathbb{U} \mathbb{E} \mathbb{H}$ with a point $Z$ on a surface of a ellipsoid and denote by $D_{e}\{Z, \hat{Z}\}=\|Z-\hat{Z}\|_{H_{N}}$ the distance between $Z$ and $\hat{Z}$ in $\mathbb{H}_{N}$.

Definition 4.7.1. The mapping $\mathcal{G}_{e}\left(\left(\overrightarrow{0}, q_{n}\right)\right)$ from $\left\{\left(\overrightarrow{0}, q_{n}\right): q_{n} \in \mathbb{U} \mathbb{E} \mathbb{H}\right\}$ into the ellipsoid is defined by

$$
\mathcal{G}_{e}\left(\left(\overrightarrow{0}, q_{n}\right)\right)= \begin{cases}\left(c_{n} u, t_{e n} q_{n}\right)=\left(\left(1-t_{e n}\right) \gamma_{e} u, t_{e n} q_{n}\right) & \text { if } q_{n} \in \mathbb{H} \\ \left(\gamma_{e} u, \psi(\infty) U\right)=\left(\gamma_{e} u, \sqrt{-\alpha_{e}} U\right) & \text { if } q_{n}=\infty U\end{cases}
$$

Proposition 4.7.1 The mapping $\mathcal{G}_{e}$ from $\left\{\left(\overrightarrow{0}, q_{n}\right): q_{n} \in \mathbb{U} \mathbb{E} \mathbb{H}\right\}$ into the ellipsoid is bijection.

\subsubsection{The Ellipsoid Metric Induced}

In this section, we develop a metric on the set $\mathbb{U E H}$ that is induced by the ellipsoid boundization

Theorem 4.7.2 the distance between any two points in $\mathbb{U E H}$ defines a metric as follows

1. Let $(\overrightarrow{0}, q)$ and $(\overrightarrow{0}, \hat{q})$ such that $q, \hat{q} \in \mathbb{H}$ then

$$
\begin{aligned}
& D_{e}^{2}\{(\overrightarrow{0}, q),(\overrightarrow{0}, \hat{q})\} \\
& =\left(\hat{t}_{e}-t_{e}\right)^{2} \gamma_{e} \bar{\gamma}_{e}+\left(t_{e}\|q\|-\hat{t}_{e}\|\hat{q}\|\right)^{2}+\hat{t}_{e} t_{e}(2\|q\|\|\hat{q}\|-\langle\hat{q}, q\rangle-\langle q, \hat{q}\rangle)
\end{aligned}
$$


2. Let $(\overrightarrow{0}, q),\left(\overrightarrow{0}, \infty U_{2}\right)$ such that $q \in \mathbb{H}$ then we have

$$
\begin{aligned}
& D_{e}^{2}\{(\overrightarrow{0}, q),(\overrightarrow{0}, \infty U)\} \\
& =t_{e}^{2} \gamma_{e} \bar{\gamma}_{e}+\left(t_{e}\|q\|-\sqrt{-\alpha_{e}}\right)^{2}+t_{e} \sqrt{-\alpha_{e}}(2\|q\|-\langle U, q\rangle-\langle q, U\rangle)
\end{aligned}
$$

3. Let $\left(\overrightarrow{0}, \infty U_{1}\right),\left(\overrightarrow{0}, \infty U_{2}\right)$ then

$$
D_{e}^{2}\left\{\left(\overrightarrow{0}, \infty U_{1}\right),\left(\overrightarrow{0}, \infty U_{2}\right)\right\}=-\alpha_{e}\left\|U_{1}-U_{2}\right\|^{2}
$$

where $U_{1}$ is a unit vector of $q$ and $U_{2}$ is a unit vector of $\hat{q}$

Moreover, the metric is continuous in $\mathbb{U} \mathbb{E} \mathbb{H}$ as follows.

$$
D_{e}^{2}\left\{(\overrightarrow{0}, q),\left(\overrightarrow{0}, \infty U_{2}\right)\right\}=\lim _{\left\|\hat{q}_{n}\right\| \longrightarrow \infty} D_{e}^{2}\left\{(\overrightarrow{0}, q),\left(\overrightarrow{0}, \hat{q}_{n}\right)\right\}
$$

and

$$
D_{e}^{2}\left\{\left(\overrightarrow{0}, \infty U_{1}\right),\left(\overrightarrow{0}, \infty U_{2}\right)\right\}=\lim _{\substack{\left\|q_{n}\right\| \longrightarrow \infty \\\left\|\hat{q}_{n}\right\| \longrightarrow \infty}} D_{e}^{2}\left\{\left(\overrightarrow{0}, q_{n}\right),\left(\overrightarrow{0}, \hat{q}_{n}\right)\right\}
$$

Proof To reach our goal, first we show that the following expression $\hat{t}_{e}-t_{e}$ has $\|q\|-\|\hat{q}\|$ as a common factor, after that it should be fairy obvious that the three axioms for a metric are satisfied in this case.

$$
\begin{aligned}
\hat{t}_{e}-t_{e} & =\frac{-\mathcal{D}+\sqrt{\hat{\mathcal{E}}}}{\|\hat{q}\|^{2}-\mathcal{D}}-\frac{-\mathcal{D}+\sqrt{\mathcal{E}}}{\|q\|^{2}-\mathcal{D}} \\
& =\frac{\left(\|q\|^{2}-\mathcal{D}\right)(-\mathcal{D}+\sqrt{\hat{\mathcal{E}}})-\left(\|\hat{q}\|^{2}-\mathcal{D}\right)(-\mathcal{D}+\sqrt{\mathcal{E}})}{\left(\|\hat{q}\|^{2}-\mathcal{D}\right)\left(\|q\|^{2}-\mathcal{D}\right)}
\end{aligned}
$$

Put $N$ for the numerator of an equation (4.7.6) so that

$$
\begin{aligned}
& N=\|q\|^{2}(-\mathcal{D})+\|q\|^{2} \sqrt{\hat{\mathcal{E}}}+\mathcal{D}^{2}-\mathcal{D} \sqrt{\hat{\mathcal{E}}} \\
& -\|\hat{q}\|^{2}(-\mathcal{D})-\|\hat{q}\|^{2} \sqrt{\mathcal{E}}-\mathcal{D}^{2}+\mathcal{D} \sqrt{\mathcal{E}} \\
& =-\|q\|^{2} \mathcal{D}+\|q\|^{2} \sqrt{\hat{\mathcal{E}}}-\mathcal{D} \sqrt{\hat{\mathcal{E}}}+\|\hat{q}\|^{2} \mathcal{D}-\|\hat{q}\|^{2} \sqrt{\mathcal{E}}+\mathcal{D} \sqrt{\mathcal{E}}
\end{aligned}
$$


add and subtract this value $\|q\|^{2} \sqrt{\mathcal{E}}$ to $N$ we obtain

$$
\begin{aligned}
& N=-\|q\|^{2} \mathcal{D}+\|q\|^{2} \sqrt{\hat{\mathcal{E}}}-\mathcal{D} \sqrt{\hat{\mathcal{E}}}+\|q\|^{2} \sqrt{\mathcal{E}}-\|q\|^{2} \sqrt{\mathcal{E}} \\
& +\|\hat{q}\|^{2} \mathcal{D}-\|\hat{q}\|^{2} \sqrt{\mathcal{E}}+\mathcal{D} \sqrt{\mathcal{E}} \\
& =\left(\|q\|^{2}-\|\hat{q}\|^{2}\right)(-\mathcal{D})+\|q\|^{2}(\sqrt{\hat{\mathcal{E}}}-\sqrt{\mathcal{E}}) \\
& +\left(\|q\|^{2}-\|\hat{q}\|^{2}\right) \sqrt{\mathcal{E}}-\mathcal{D}(\sqrt{\hat{\mathcal{E}}}-\sqrt{\mathcal{E}}) \\
& =\left(\|q\|^{2}-\|\hat{q}\|^{2}\right)(-\mathcal{D}+\sqrt{\mathcal{E}})+\left(\|q\|^{2}-\mathcal{D}\right)(\sqrt{\hat{\mathcal{E}}}-\sqrt{\mathcal{E}})
\end{aligned}
$$

thus

$$
\hat{t}_{e}-t_{e}=\frac{\left(\|q\|^{2}-\|\hat{q}\|^{2}\right)(-\mathcal{D}+\sqrt{\mathcal{E}})+\left(\|q\|^{2}-\mathcal{D}\right)(\sqrt{\hat{\mathcal{E}}}-\sqrt{\mathcal{E}})}{\left(\|\hat{q}\|^{2}-\mathcal{D}\right)\left(\|q\|^{2}-\mathcal{D}\right)}
$$

Some simplification is needed, we use a simple trick worth remembering, we multiply the entire expression of $\hat{t}_{e}-t_{e}$ by this value $\frac{\sqrt{\hat{\mathcal{E}}}+\sqrt{\mathcal{E}}}{\sqrt{\hat{\mathcal{E}}}+\sqrt{\mathcal{E}}}$ which is convenient to simplify the expression to the following equation

$$
\begin{aligned}
& \hat{t}_{e}-t_{e} \\
& =\frac{\left(\|q\|^{2}-\|\hat{q}\|^{2}\right)(-\mathcal{D}+\sqrt{\mathcal{E}})(\sqrt{\hat{\mathcal{E}}}+\sqrt{\mathcal{E}})+\left(\|q\|^{2}-\mathcal{D}\right)(\hat{\mathcal{E}}-\mathcal{E})}{\left(\|\hat{q}\|^{2}-\mathcal{D}\right)\left(\|q\|^{2}-\mathcal{D}\right)(\sqrt{\hat{\mathcal{E}}}+\sqrt{\mathcal{E}})}
\end{aligned}
$$

we proceed to find a form $\hat{\mathcal{E}}-\mathcal{E}$ as follows

$$
\hat{\mathcal{E}}-\mathcal{E}=\mathcal{D}^{2}-\left(\|\hat{q}\|^{2}-\mathcal{D}\right) \alpha_{h}-\mathcal{D}^{2}+\left(\|q\|^{2}-\mathcal{D}\right) \alpha_{e}=-\alpha_{e}\left(\|\hat{q}\|^{2}-\|q\|^{2}\right)
$$

from the equation (4.7.8) we obtain

$$
\hat{t}_{e}-t_{e}=\frac{\left(\|q\|^{2}-\|\hat{q}\|^{2}\right)\left[(-\mathcal{D}+\sqrt{\mathcal{E}})(\sqrt{\hat{\mathcal{E}}}+\sqrt{\mathcal{E}})+\left(\|q\|^{2}-\mathcal{D}\right) \alpha_{e}\right]}{\left(\|\hat{q}\|^{2}-\mathcal{D}\right)\left(\|q\|^{2}-\mathcal{D}\right)(\sqrt{\hat{\mathcal{E}}}+\sqrt{\mathcal{E}})}
$$

The first two conditions in the definition of a metric are obviously satisfied, namely for positivity $D_{e}$ is nonnegative number $D_{e}\{(\overrightarrow{0}, q),(\overrightarrow{0}, \hat{q})\} \geq 0$ 
and by (4.7.9) $D_{e}$ only be equal to zero if and only if when $q=\hat{q}$ or when $\|q\|=\|\hat{q}\|$.

$$
D_{e}\{(\overrightarrow{0}, q),(\overrightarrow{0}, \hat{q})\}=0 \text { if and only if } q=\hat{q} \text { or }\|q\|=\|\hat{q}\| .
$$

And for symmetry we have

$D_{e}\{(\overrightarrow{0}, q),(\overrightarrow{0}, \hat{q})\}=\|(\overrightarrow{0}, q)-(\overrightarrow{0}, \hat{q})\|_{H_{N}}=\|(\overrightarrow{0}, \hat{q})-(\overrightarrow{0}, q)\|_{H_{N}}=D_{e}\{(\overrightarrow{0}, \hat{q}),(\overrightarrow{0}, q)\}$

To prove the triangle inequality, observe for any third point in $U E H(\overrightarrow{0}, q)$, $(\overrightarrow{0}, \hat{q})$ and $(\overrightarrow{0}, \tilde{q})$ we have

$$
\begin{aligned}
D_{e}\{(\overrightarrow{0}, q),(\overrightarrow{0}, \hat{q})\} & =\|(\overrightarrow{0}, q)-(\overrightarrow{0}, \hat{q})\|_{H_{N}} \\
& =\|(\overrightarrow{0}, q)-(\overrightarrow{0}, \tilde{q})+(\overrightarrow{0}, \tilde{q})-(\overrightarrow{0}, \hat{q})\|_{H_{N}} \\
& \leq\|(\overrightarrow{0}, q)-(\overrightarrow{0}, \tilde{q})\|_{H_{N}}+\|(\overrightarrow{0}, \tilde{q})-(\overrightarrow{0}, \hat{q})\|_{H_{N}} \\
& \leq D_{e}\{(\overrightarrow{0}, q),(\overrightarrow{0}, \tilde{q})\}+D_{e}\{(\overrightarrow{0}, \tilde{q}),(\overrightarrow{0}, \hat{q})\}
\end{aligned}
$$

Moreover,

$$
D_{e}^{2}\{(\overrightarrow{0}, q),(\overrightarrow{0}, \hat{q})\}=\left(\hat{t}_{e}-t_{e}\right)^{2} \gamma_{e} \bar{\gamma}_{e}+\left\langle t_{e} q-\hat{t}_{e} \hat{q}, t_{e} q-\hat{t}_{e} \hat{q}\right\rangle
$$

by the proposition (3.9.2) we have $\hat{t}_{e n} \hat{q}_{n} \sim \sqrt{-\alpha_{e}} U_{2}$ as $n \longrightarrow \infty$ and by the definition 4.7.1 we have $\hat{t}_{e} \hat{q}=\sqrt{-\alpha_{e}} U_{2}$ and as we know that when $\|\hat{q}\| \longrightarrow \infty$ then $\hat{t}_{s} \longrightarrow 0$ thus the equation (4.7.10) becomes

$$
D_{e}^{2}\left\{(\overrightarrow{0}, q),\left(\overrightarrow{0}, \infty U_{2}\right)\right\}=t_{e}^{2} \gamma_{e} \bar{\gamma}_{e}+\left\langle t_{e} q-\sqrt{-\alpha_{e}} U_{2}, t_{e} q-\sqrt{-\alpha_{e}} U_{2}\right\rangle
$$

therefore we get the equation (4.7.2). And when $\|q\| \longrightarrow \infty$ and $\|\hat{q}\| \longrightarrow \infty$ in the equation (4.7.1) we will get the equation (4.7.3) as follows. By propositions 3.9.2 and 3.9.3 we have $t_{e n} q_{n} \sim \sqrt{-\alpha_{e}} U_{1}$ and $\hat{t}_{e n} \hat{q}_{n} \sim \sqrt{-\alpha_{e}} U_{2}$ as $n \longrightarrow$ $\infty$ and by the definition 4.7 .1 we have $t_{e} q=\sqrt{-\alpha_{e}} U_{1}$ and $\hat{t}_{e} \hat{q}=\sqrt{-\alpha_{e}} U_{2}$ also as we know when $\|q\| \longrightarrow \infty$ then $t_{e} \longrightarrow 0$ and when $\|\hat{q}\| \longrightarrow \infty$ then $\hat{t}_{e} \longrightarrow 0$ therefore the equation (4.7.10) becomes

$$
D_{e}^{2}\left\{\left(\overrightarrow{0}, \infty U_{2}\right),\left(\overrightarrow{0}, \infty U_{2}\right)\right\}=\left\langle\sqrt{-\alpha_{e}} U_{1}-\sqrt{-\alpha_{e}} U_{2} \sqrt{-\alpha_{e}} U_{1}-\sqrt{-\alpha_{e}} U_{2}\right\rangle
$$

therefore we get (4.7.3) 


\section{Chapter 5}

\section{Equivalence and Nonequivalence Between Extended Metrics}

\subsection{Metrics Nonequivalence and Metrics Equiv- alence}

The various boundizations proposed and studied in this disseration may be thought of as conic sections in the plane that are extended to Hilbert or Banach spaces. The boundizations manifest themself geometrically as surfaces in these function spaces. These surfaces are naturally characterized in Hilbert or Banach spaces, analogous to their characterization in a plane, by certain distances from one or two foci and by a distances from an object like a point a line or a plane. The measure of a distance emanating from the norm with which a given functional space is endowed. The richness of the different boundizations and the dependence of each on several parameters serve a few purposes. They provide a setting for better understanding the nature of boundizations and how does one boundization differs from another. Moreover, a researcher in the mathematical sciences is given a larger pool of boundizations to choose from to better fit his/ her specific scientific settings and goals. This can come in handy for example in approximation theory. It then goes without saying that it is of paramount importance to study criteria which will determine whether two different metrics are equivalent or not. It is well known, see e.g. Kreitzig [35] Chapter two. page 75, that any 
two norms in a finite dimensional Banach space are equivalent. It was then unexpected to discover that certain boundizations, like the cone boundization and the sphere boundization, that are widely used in the mathematical sciences induce metrics that are not equivalent. One should not confuse the equivalence or non equivalence of the metric induced by boundizations in a finite dimensional Banach space with the norm with which a Banach space is endowed. The reason being that the metrics that are subject to study in this dissertation are defined in an Extended Banach Space that is obtained by non linear mappings and therefore they not obey all the axioms of a linear Banach space.

\subsection{General Theorems for Metrics}

As there could be more than one metric defined on the Ultra Extended Hilbert space, we study the problem whether or not some of these metrics are equivalent. To this end we offer some general theorems and lemmas. Let $D_{g_{1}}$ and $D_{g_{2}}$ be two metrics induced by boundizations as given in definition of boundization, we will study in the sequel under what condition $D_{g_{1}}$ and $D_{g_{2}}$ are equivalent. Recall the definition of the equivalence of two metrics on a given space, and see e.g. [50] chapter 9 page 185 . To this end we need to estimate the quotient $\frac{D_{g_{1}}}{D_{g_{2}}}$ for all $0 \leq\|q\| \leq \infty$. First we assert the following general theorems for equivalence between two metrics. We also need the concept of subordination given in the next definition.

Definition 5.2.1 The metric $D^{2}[(\overrightarrow{0}, q),(\overrightarrow{0}, \hat{q})]$ is subordinate to the metric $\tilde{D}^{2}[(\overrightarrow{0}, q),(\overrightarrow{0}, \hat{q})]$ if there exists a constant $C>0$ such that

$$
D^{2}[(\overrightarrow{0}, q),(\overrightarrow{0}, \hat{q})] \leq C \tilde{D}^{2}[(\overrightarrow{0}, q),(\overrightarrow{0}, \hat{q})],
$$

where $q, \hat{q} \in \mathbb{U} \mathbb{E} \mathbb{H}$.

We proceed with a few general theorems about the equivalence of metrics induced on $\mathbb{H}_{N}$.

Theorem 5.2.1 Assume that $D_{g_{1}}$ and $D_{g_{2}}$ be two metrics induced by boundizations and given by

$$
\begin{aligned}
& D_{g_{1}}^{2}\{(\overrightarrow{0}, q),(\overrightarrow{0}, \hat{q})\} \\
& =\left(\hat{t}_{g_{1}}-t_{g_{1}}\right)^{2} \gamma_{g_{1}} \bar{\gamma}_{g_{1}}+\left(t_{g_{1}}\|q\|-\hat{t}_{g_{1}}\|\hat{q}\|\right)^{2}+\hat{t}_{g_{1}} t_{g_{1}}(2\|q\|\|\hat{q}\|-\langle\hat{q}, q\rangle-\langle q, \hat{q}\rangle)
\end{aligned}
$$


and

$$
\begin{aligned}
& D_{g_{2}}^{2}\{(\overrightarrow{0}, q),(\overrightarrow{0}, \hat{q})\} \\
& =\left(\hat{t}_{g_{2}}-t_{g_{2}}\right)^{2} \gamma_{g_{2}} \bar{\gamma}_{g_{2}}+\left(t_{g_{2}}\|q\|-\hat{t}_{g_{2}}\|\hat{q}\|\right)^{2}+\hat{t}_{g_{2}} t_{g_{2}}(2\|q\|\|\hat{q}\|-\langle\hat{q}, q\rangle-\langle q, \hat{q}\rangle),
\end{aligned}
$$

where $\gamma_{g_{1}} \neq 0$ and $\gamma_{g_{2}} \neq 0$. Then $D_{g_{1}}$ and $D_{g_{2}}$ are equivalent if the following quantities are bounded above and below, namely

$$
0<\mathcal{N}_{1} \leq \frac{\left(\hat{t}_{g_{1}}-t_{g_{1}}\right)^{2} \gamma_{g_{1}} \bar{\gamma}_{g_{1}}}{\left(\hat{t}_{g_{2}}-t_{g_{2}}\right)^{2} \gamma_{g_{2}} \bar{\gamma}_{g_{2}}} \leq \mathcal{M}_{1}<\infty
$$

and

$$
0<\mathcal{N}_{2} \leq \frac{\left(t_{g_{1}}\|q\|-\hat{t}_{g_{1}}\|\hat{q}\|\right)^{2}}{\left(t_{g_{2}}\|q\|-\hat{t}_{g_{2}}\|\hat{q}\|\right)^{2}} \leq \mathcal{M}_{2}<\infty
$$

where $\mathcal{N}_{1}, \mathcal{M}_{1}, \mathcal{N}_{2}$ and $\mathcal{M}_{2}$ are positive real numbers.

Proof Since the quantities in equation (5.2.3) are bounded then we have

$$
\mathcal{N}_{1}\left(\hat{t}_{g_{2}}-t_{g_{2}}\right)^{2} \gamma_{g_{2}} \bar{\gamma}_{g_{2}} \leq\left(\hat{t}_{g_{1}}-t_{g_{1}}\right)^{2} \gamma_{g_{1}} \bar{\gamma}_{g_{1}} \leq\left(\hat{t}_{g_{2}}-t_{g_{2}}\right)^{2} \gamma_{g_{2}} \bar{\gamma}_{g_{2}} \mathcal{M}_{1}
$$

Assume $\hat{t}_{g_{1}}=0$ and $\hat{t}_{g_{2}}=0$ in the equation (5.2.3) we get

$$
\mathcal{N}_{1} \leq \frac{t_{g_{1}}}{t_{g_{2}}} \leq \mathcal{M}_{1}
$$

implies that

$$
\mathcal{N}_{1} t_{g_{2}} \leq t_{g_{1}} \leq t_{g_{2}} \mathcal{M}_{1}
$$

also, assume $t_{g_{1}}=0$ and $t_{g_{2}}=0$ in the equation (5.2.3) we get

$$
\mathcal{N}_{1} \leq \frac{\hat{t}_{g_{1}}}{\hat{t}_{g_{2}}} \leq \mathcal{M}_{1}
$$

implies that

$$
\mathcal{N}_{1} \hat{t}_{g_{2}} \leq \hat{t}_{g_{1}} \leq \hat{t}_{g_{2}} \mathcal{M}_{1}
$$

And by our assumption (5.2.4) we have

$$
\mathcal{N}_{2}\left(t_{g_{2}}\|q\|-\hat{t}_{g_{2}}\|\hat{q}\|\right)^{2} \leq\left(t_{g_{1}}\|q\|-\hat{t}_{g_{1}}\|\hat{q}\|\right)^{2} \leq\left(t_{g_{2}}\|q\|-\hat{t}_{g_{2}}\|\hat{q}\|\right)^{2} \mathcal{M}_{2}
$$


therefore

$$
\begin{aligned}
& \frac{D_{g_{1}}^{2}\{(\overrightarrow{0}, q),(\overrightarrow{0}, \hat{q})\}}{D_{g_{2}}^{2}\{(\overrightarrow{0}, q),(\overrightarrow{0}, \hat{q})\}}= \\
& \frac{\left(\hat{t}_{g_{1}}-t_{g_{1}}\right)^{2} \gamma_{g_{1}} \bar{\gamma}_{g_{1}}+\left(t_{g_{1}}\|q\|-\hat{t}_{g_{1}}\|\hat{q}\|\right)^{2}+\hat{t}_{g_{1}} t_{g_{1}}(2\|q\|\|\hat{q}\|-\langle\hat{q}, q\rangle-\langle q, \hat{q}\rangle)}{\left(\hat{t}_{g_{2}}-t_{g_{2}}\right)^{2} \gamma_{g_{2}} \bar{\gamma}_{g_{2}}+\left(t_{g_{2}}\|q\|-\hat{t}_{g_{2}}\|\hat{q}\|\right)^{2}+\hat{t}_{g_{2}} t_{g_{2}}(2\|q\|\|\hat{q}\|-\langle\hat{q}, q\rangle-\langle q, \hat{q}\rangle)} \\
& \frac{\mathcal{N}_{1}\left(\hat{t}_{g_{2}}-t_{g_{2}}\right)^{2} \gamma_{g_{2}} \bar{\gamma}_{g_{2}}+\mathcal{N}_{2}\left(t_{g_{2}}\|q\|-\hat{t}_{g_{2}}\|\hat{q}\|\right)^{2}+\mathcal{N}_{1}^{2} \hat{t}_{g_{2}} t_{g_{2}}(2\|q\|\|\hat{q}\|-\langle\hat{q}, q\rangle-\langle q, \hat{q}\rangle)}{\left(\hat{t}_{g_{2}}-t_{g_{2}}\right)^{2} \gamma_{g_{2}} \bar{\gamma}_{g_{2}}+\left(t_{g_{2}}\|q\|-\hat{t}_{g_{2}}\|\hat{q}\|\right)^{2}+\hat{t}_{g_{2}} t_{g_{2}}(2\|q\|\|\hat{q}\|-\langle\hat{q}, q\rangle-\langle q, \hat{q}\rangle)}
\end{aligned}
$$

let $\mathcal{K}_{1}=\operatorname{mim}\left\{\mathcal{N}_{1}, \mathcal{N}_{2}, \mathcal{N}_{1}^{2}\right\}$ then we have

$$
\begin{aligned}
& \frac{D_{g_{1}}^{2}\{(\overrightarrow{0}, q),(\overrightarrow{0}, \hat{q})\}}{D_{g_{2}}^{2}\{(\overrightarrow{0}, q),(\overrightarrow{0}, \hat{q})\}} \geq \\
& \frac{\mathcal{K}_{1}\left(\hat{t}_{g_{2}}-t_{g_{2}}\right)^{2} \gamma_{g_{2}} \bar{\gamma}_{g_{2}}+\mathcal{K}_{1}\left(t_{g_{2}}\|q\|-\hat{t}_{g_{2}}\|\hat{q}\|\right)^{2}+\mathcal{K}_{1} \hat{t}_{g_{2}} t_{g_{2}}(2\|q\|\|\hat{q}\|-\langle\hat{q}, q\rangle-\langle q, \hat{q}\rangle)}{\left(\hat{t}_{g_{2}}-t_{g_{2}}\right)^{2} \gamma_{g_{2}} \bar{\gamma}_{g_{2}}+\left(t_{g_{2}}\|q\|-\hat{t}_{g_{2}}\|\hat{q}\|\right)^{2}+\hat{t}_{g_{2}} t_{g_{2}}(2\|q\|\|\hat{q}\|-\langle\hat{q}, q\rangle-\langle q, \hat{q}\rangle)} \\
& =\mathcal{K}_{1}
\end{aligned}
$$

Also

$$
\begin{aligned}
& \frac{D_{g_{1}}^{2}\{(\overrightarrow{0}, q),(\overrightarrow{0}, \hat{q})\}}{D_{g_{2}}^{2}\{(\overrightarrow{0}, q),(\overrightarrow{0}, \hat{q})\}} \\
& =\frac{\left(\hat{t}_{g_{1}}-t_{g_{1}}\right)^{2} \gamma_{g_{1}} \bar{\gamma}_{g_{1}}+\left(t_{g_{1}}\|q\|-\hat{t}_{g_{1}}\|\hat{q}\|\right)^{2}+\hat{t}_{g_{1}} t_{g_{1}}(2\|q\|\|\hat{q}\|-\langle\hat{q}, q\rangle-\langle q, \hat{q}\rangle)}{\left(\hat{t}_{g_{2}}-t_{g_{2}}\right)^{2} \gamma_{g_{2}} \bar{\gamma}_{g_{2}}+\left(t_{g_{2}}\|q\|-\hat{t}_{g_{2}}\|\hat{q}\|\right)^{2}+\hat{t}_{g_{2}} t_{g_{2}}(2\|q\|\|\hat{q}\|-\langle\hat{q}, q\rangle-\langle q, \hat{q}\rangle)} \leq \\
& \frac{\mathcal{M}_{1}\left(\hat{t}_{g_{2}}-t_{g_{2}}\right)^{2} \gamma_{g_{2}} \bar{\gamma}_{g_{2}}+\mathcal{M}_{2}\left(t_{g_{2}}\|q\|-\hat{t}_{g_{2}}\|\hat{q}\|\right)^{2}+\mathcal{M}_{1}^{2} \hat{t}_{g_{2}} t_{g_{2}}(2\|q\|\|\hat{q}\|-\langle\hat{q}, q\rangle-\langle q, \hat{q}\rangle)}{\left(\hat{t}_{g_{2}}-t_{g_{2}}\right)^{2} \gamma_{g_{2}} \bar{\gamma}_{g_{2}}+\left(t_{g_{2}}\|q\|-\hat{t}_{g_{2}}\|\hat{q}\|\right)^{2}+\hat{t}_{g_{2}} t_{g_{2}}(2\|q\|\|\hat{q}\|-\langle\hat{q}, q\rangle-\langle q, \hat{q}\rangle)}
\end{aligned}
$$

let $\mathcal{K}_{2}=\operatorname{Max}\left\{\mathcal{M}_{1}, \mathcal{M}_{2}, \mathcal{M}_{1}^{2}\right\}$ then we have

$$
\begin{aligned}
& \frac{D_{g_{1}}^{2}\{(\overrightarrow{0}, q),(\overrightarrow{0}, \hat{q})\}}{D_{g_{2}}^{2}\{(\overrightarrow{0}, q),(\overrightarrow{0}, \hat{q})\}} \leq \\
& \frac{\mathcal{K}_{2}\left(\hat{t}_{g_{2}}-t_{g_{2}}\right)^{2} \gamma_{g_{2}} \bar{\gamma}_{g_{2}}+\mathcal{K}_{2}\left(t_{g_{2}}\|q\|-\hat{t}_{g_{2}}\|\hat{q}\|\right)^{2}+\mathcal{K}_{2} \hat{t}_{g_{2}} t_{g_{2}}(2\|q\|\|\hat{q}\|-\langle\hat{q}, q\rangle-\langle q, \hat{q}\rangle)}{\left(\hat{t}_{g_{2}}-t_{g_{2}}\right)^{2} \gamma_{g_{2}} \bar{\gamma}_{g_{2}}+\left(t_{g_{2}}\|q\|-\hat{t}_{g_{2}}\|\hat{q}\|\right)^{2}+\hat{t}_{g_{2}} t_{g_{2}}(2\|q\|\|\hat{q}\|-\langle\hat{q}, q\rangle-\langle q, \hat{q}\rangle)} \\
& =\mathcal{K}_{2}
\end{aligned}
$$


Thus

$$
\mathcal{K}_{1} \leq \frac{D_{g_{1}}^{2}\{(\overrightarrow{0}, q),(\overrightarrow{0}, \hat{q})\}}{D_{g_{2}}^{2}\{(\overrightarrow{0}, q),(\overrightarrow{0}, \hat{q})\}} \leq \mathcal{K}_{2}
$$

The proof is complete.

Theorem 5.2.2 Assume that $D_{g_{1}}$ and $D_{g_{2}}$ are two metrics induced by boundizations subject to (5.2.1) and (5.2.2). Then $D_{g_{1}}$ and $D_{g_{2}}$ are equivalent if the following quantities are bounded above and below, namely

$$
0<\mathcal{N}_{3} \leq \frac{\left(\hat{t}_{g_{1}}-t_{g_{1}}\right)^{2} \gamma_{g_{1}} \bar{\gamma}_{g_{1}}+\left(t_{g_{1}}\|q\|-\hat{t}_{g_{1}}\|\hat{q}\|\right)^{2}}{\left(\hat{t}_{g_{2}}-t_{g_{2}}\right)^{2} \gamma_{g_{2}} \bar{\gamma}_{g_{2}}+\left(t_{g_{2}}\|q\|-\hat{t}_{g_{2}}\|\hat{q}\|\right)^{2}} \leq \mathcal{M}_{3}<\infty
$$

and

$$
0<\mathcal{N}_{4}^{2} \leq \frac{\hat{t}_{g_{1}} t_{g_{1}}}{\hat{t}_{g_{2}} t_{g_{2}}} \leq \mathcal{M}_{4}^{2}
$$

where $\mathcal{N}_{3}, \mathcal{M}_{3}, \mathcal{N}_{4}$ and $\mathcal{M}_{4}$ are positive real numbers.

Proof By our assumptions we have

$$
\begin{aligned}
& \frac{D_{g_{1}}^{2}\{(\overrightarrow{0}, q),(\overrightarrow{0}, \hat{q})\}}{D_{g_{2}}^{2}\{(\overrightarrow{0}, q),(\overrightarrow{0}, \hat{q})\}} \\
& =\frac{\left(\hat{t}_{g_{1}}-t_{g_{1}}\right)^{2} \gamma_{g_{1}} \bar{\gamma}_{g_{1}}+\left(t_{g_{1}}\|q\|-\hat{t}_{g_{1}}\|\hat{q}\|\right)^{2}+\hat{t}_{g_{1}} t_{g_{1}}(2\|q\|\|\hat{q}\|-\langle\hat{q}, q\rangle-\langle q, \hat{q}\rangle)}{\left(\hat{t}_{g_{2}}-t_{g_{2}}\right)^{2} \gamma_{g_{2}} \bar{\gamma}_{g_{2}}+\left(t_{g_{2}}\|q\|-\hat{t}_{g_{2}}\|\hat{q}\|\right)^{2}+\hat{t}_{c} t_{c}(2\|q\|\|\hat{q}\|-\langle\hat{q}, q\rangle-\langle q, \hat{q}\rangle)} \\
& \geq \frac{\mathcal{N}_{3}\left(\left(\hat{t}_{g_{2}}-t_{g_{2}}\right)^{2} \gamma_{g_{2}} \bar{\gamma}_{g_{2}}+\left(t_{g_{2}}\|q\|-\hat{t}_{g_{2}}\|\hat{q}\|\right)^{2}\right)}{\left(\hat{t}_{g_{2}}-t_{g_{2}}\right)^{2} \gamma_{g_{2}} \bar{\gamma}_{c}+\left(t_{c}\|q\|-\hat{t}_{c}\|\hat{q}\|\right)^{2}+\hat{t}_{g_{2}} t_{g_{2}}(2\|q\|\|\hat{q}\|-\langle\hat{q}, q\rangle-\langle q, \hat{q}\rangle)} \\
& +\frac{\mathcal{N}_{4}^{2} \hat{t}_{g_{2}} t_{g_{2}}(2\|q\|\|\hat{q}\|-\langle\hat{q}, q\rangle-\langle q, \hat{q}\rangle)}{\left(\hat{t}_{g_{2}}-t_{g_{2}}\right)^{2} \gamma_{g_{2}} \bar{\gamma}_{c}+\left(t_{c}\|q\|-\hat{t}_{c}\|\hat{q}\|\right)^{2}+\hat{t}_{g_{2}} t_{g_{2}}(2\|q\|\|\hat{q}\|-\langle\hat{q}, q\rangle-\langle q, \hat{q}\rangle)}
\end{aligned}
$$


suppose $\mathcal{K}_{3}=\operatorname{mim}\left\{\mathcal{N}_{3}, \mathcal{N}_{4}^{2}\right\}$ thus

$$
\begin{aligned}
& \frac{D_{g_{1}}^{2}\{(\overrightarrow{0}, q),(\overrightarrow{0}, \hat{q})\}}{D_{g_{2}}^{2}\{(\overrightarrow{0}, q),(\overrightarrow{0}, \hat{q})\}} \\
& \geq \frac{\mathcal{K}_{3}\left(\left(\hat{t}_{g_{2}}-t_{g_{2}}\right)^{2} \gamma_{g_{2}} \bar{\gamma}_{g_{2}}+\left(t_{g_{2}}\|q\|-\hat{t}_{g_{2}}\|\hat{q}\|\right)^{2}\right)}{\left(\hat{t}_{g_{2}}-t_{g_{2}}\right)^{2} \gamma_{g_{2}} \bar{\gamma}_{c}+\left(t_{c}\|q\|-\hat{t}_{c}\|\hat{q}\|\right)^{2}+\hat{t}_{g_{2}} t_{g_{2}}(2\|q\|\|\hat{q}\|-\langle\hat{q}, q\rangle-\langle q, \hat{q}\rangle)} \\
& +\frac{\mathcal{K}_{3}^{2} \hat{t}_{g_{2}} t_{g_{2}}(2\|q\|\|\hat{q}\|-\langle\hat{q}, q\rangle-\langle q, \hat{q}\rangle)}{\left(\hat{t}_{g_{2}}-t_{g_{2}}\right)^{2} \gamma_{g_{2}} \bar{\gamma}_{c}+\left(t_{c}\|q\|-\hat{t}_{c}\|\hat{q}\|\right)^{2}+\hat{t}_{g_{2}} t_{g_{2}}(2\|q\|\|\hat{q}\|-\langle\hat{q}, q\rangle-\langle q, \hat{q}\rangle)} \\
& =\mathcal{K}_{3}
\end{aligned}
$$

Also

$$
\begin{aligned}
& \frac{D_{g_{1}}^{2}\{(\overrightarrow{0}, q),(\overrightarrow{0}, \hat{q})\}}{D_{g_{2}}^{2}\{(\overrightarrow{0}, q),(\overrightarrow{0}, \hat{q})\}} \\
& \leq \frac{\mathcal{M}_{4}\left(\left(\hat{t}_{g_{2}}-t_{g_{2}}\right)^{2} \gamma_{g_{2}} \bar{\gamma}_{g_{2}}+\left(t_{g_{2}}\|q\|-\hat{t}_{g_{2}}\|\hat{q}\|\right)^{2}\right)}{\left(\hat{t}_{g_{2}}-t_{g_{2}}\right)^{2} \gamma_{g_{2}} \bar{\gamma}_{c}+\left(t_{c}\|q\|-\hat{t}_{c}\|\hat{q}\|\right)^{2}+\hat{t}_{g_{2}} t_{g_{2}}(2\|q\|\|\hat{q}\|-\langle\hat{q}, q\rangle-\langle q, \hat{q}\rangle)} \\
& +\frac{\mathcal{M}_{4}^{2} \hat{t}_{g_{2}} t_{g_{2}}(2\|q\|\|\hat{q}\|-\langle\hat{q}, q\rangle-\langle q, \hat{q}\rangle)}{\left(\hat{t}_{g_{2}}-t_{g_{2}}\right)^{2} \gamma_{g_{2}} \bar{\gamma}_{c}+\left(t_{c}\|q\|-\hat{t}_{c}\|\hat{q}\|\right)^{2}+\hat{t}_{g_{2}} t_{g_{2}}(2\|q\|\|\hat{q}\|-\langle\hat{q}, q\rangle-\langle q, \hat{q}\rangle)}
\end{aligned}
$$

suppose $\mathcal{K}_{4}=\operatorname{Max}\left\{\mathcal{M}_{4}, \mathcal{M}_{4}^{2}\right\}$ thus

$$
\begin{aligned}
& \frac{D_{g_{1}}^{2}\{(\overrightarrow{0}, q),(\overrightarrow{0}, \hat{q})\}}{D_{g_{2}}^{2}\{(\overrightarrow{0}, q),(\overrightarrow{0}, \hat{q})\}} \\
& \leq \frac{\mathcal{K}_{4}\left(\left(\hat{t}_{g_{2}}-t_{g_{2}}\right)^{2} \gamma_{g_{2}} \bar{\gamma}_{g_{2}}+\left(t_{g_{2}}\|q\|-\hat{t}_{g_{2}}\|\hat{q}\|\right)^{2}\right)}{\left(\hat{t}_{g_{2}}-t_{g_{2}}\right)^{2} \gamma_{g_{2}} \bar{\gamma}_{c}+\left(t_{c}\|q\|-\hat{t}_{c}\|\hat{q}\|\right)^{2}+\hat{t}_{g_{2}} t_{g_{2}}(2\|q\|\|\hat{q}\|-\langle\hat{q}, q\rangle-\langle q, \hat{q}\rangle)} \\
& +\frac{\mathcal{K}_{4}^{2} \hat{t}_{g_{2}} t_{g_{2}}(2\|q\|\|\hat{q}\|-\langle\hat{q}, q\rangle-\langle q, \hat{q}\rangle)}{\left(\hat{t}_{g_{2}}-t_{g_{2}}\right)^{2} \gamma_{g_{2}} \bar{\gamma}_{c}+\left(t_{c}\|q\|-\hat{t}_{c}\|\hat{q}\|\right)^{2}+\hat{t}_{g_{2}} t_{g_{2}}(2\|q\|\|\hat{q}\|-\langle\hat{q}, q\rangle-\langle q, \hat{q}\rangle)} \\
& =\mathcal{K}_{4}
\end{aligned}
$$

The proof is complete.

In what follows we prove lemmas the motivation of which will become apparent later on. 
Lemma 5.2.3 Assume

1. $q \in \mathbb{H}$.

2. $\delta_{1}>0, \delta_{2}, \delta_{3}$ and $\delta_{4}>0$ are any real numbers, with $\delta_{2}^{2}+\delta_{3} \delta_{4}>0$ and $\sqrt{\delta_{2}^{2}+\delta_{3} \delta_{4}}+\delta_{2}>0$

Then the quantity

$$
\frac{\|q\|+\delta_{1}}{\sqrt{\delta_{2}^{2}+\left(\|q\|^{2}+\delta_{3}\right) \delta_{4}}+\delta_{2}}
$$

is bounded above and below.

Proof Note that the quotient in (5.2.13) is a continuous function of $\|q\|$ on any finite interval $0 \leq\|q\| \leq \hat{\rho}$, where $\hat{\rho}>0$ is fixed. By our assumption (ii) the denominator is bounded away from zero. Next we will show that the quotient in (5.2.13) is bounded above and below on any interval $\tilde{\rho} \leq\|q\|<\infty$ by computing the limit of the quantity as $\|q\| \longrightarrow \infty$, and then we use the definition of the limit.

$$
\begin{aligned}
& \lim _{\|q\| \rightarrow \infty} \frac{\|q\|+\delta_{1}}{\sqrt{\delta_{2}^{2}+\left(\|q\|^{2}+\delta_{3}\right) \delta_{4}}+\delta_{2}} \\
& =\lim _{\|q\| \rightarrow \infty} \frac{1+\frac{\delta_{1}}{\|q\|}}{\sqrt{\frac{\delta_{1}}{\|q\|^{2}}+\left(1+\frac{\delta_{3}^{2}}{\|q\|^{2}}\right) \delta_{4}}+\frac{\delta_{2}}{\|q\|}}=\frac{1}{\sqrt{\delta_{4}}}
\end{aligned}
$$

By definition of a limit we have for every $\epsilon>0$, then there exists $\rho>0$ such that for all $\|q\| \geq \rho$. we have

$$
\frac{1}{\sqrt{\delta_{4}}}-\epsilon \leq \frac{\|q\|+\delta_{1}}{\sqrt{\delta_{2}^{2}+\left(\|q\|^{2}+\delta_{3}\right) \delta_{4}}+\delta_{2}} \leq \frac{1}{\sqrt{\delta_{4}}}+\epsilon
$$

Choose $\epsilon=\frac{1}{2 \sqrt{\delta_{4}}}$. Moreover the numerator and the denominator in (5.2.13) are positive and bounded on $[\hat{\rho}, \tilde{\rho}]$ and their quotient is a continuous function of $\|q\|$ on the closed interval $[\hat{\rho}, \tilde{\rho}]$. This implies that the quotient has a positive minimum and maximum on $[\hat{\rho}, \tilde{\rho}]$. The proof is complete.

Next we need the following lemma.

Lemma 5.2.4 Assume 
1. $q \in \mathbb{H}$.

2. $\delta_{4}>0, \delta_{2}$ and $\delta_{3}$ are any real numbers, with $\delta_{2}^{2}+\delta_{3} \delta_{4}>0$

then the quantity

$$
\frac{\|q\|+\|\hat{q}\|}{\sqrt{\delta_{2}^{2}+\left(\|q\|^{2}+\delta_{3}\right) \delta_{4}}+\sqrt{\delta_{2}^{2}+\left(\|\hat{q}\|^{2}+\delta_{3}\right) \delta_{4}}}
$$

is bounded above, namely

$$
0 \leq \frac{\|q\|+\|\hat{q}\|}{\sqrt{\delta_{2}^{2}+\left(\|q\|^{2}+\delta_{3}\right) \delta_{4}}+\sqrt{\delta_{2}^{2}+\left(\|\hat{q}\|^{2}+\delta_{3}\right) \delta_{4}}} \leq \mathcal{M}_{8}<\infty
$$

where is a cretin positive real number.

Proof Consider

$$
T(\|q\|)=\frac{\|q\|}{\sqrt{\delta_{2}^{2}+\left(\|q\|^{2}+\delta_{3}\right) \delta_{4}}}
$$

then

$$
\lim _{\|q\| \longrightarrow \infty} T(\|q\|)=\lim _{\|q\| \rightarrow \infty} \frac{\|q\|}{\sqrt{\delta_{2}^{2}+\left(\|q\|^{2}+\delta_{3}\right) \delta_{4}}}=\frac{1}{\sqrt{\delta_{4}}} \neq 0
$$

By definition of the limit given any $\epsilon>0$, there exist $\rho>0$ such that for all $\|q\| \leq \rho$ we have

$$
\frac{1}{\sqrt{\delta_{4}}}-\epsilon \leq \frac{\|q\|}{\sqrt{\delta_{2}^{2}+\left(\|q\|^{2}+\delta_{3}\right) \delta_{4}}} \leq \frac{1}{\sqrt{\delta_{4}}}+\epsilon
$$

Thus the quantity $(\|q\|)$ is bounded on $[\tilde{\rho}, \infty)$. Namely,

$$
\frac{\|q\|}{\sqrt{\delta_{2}^{2}+\left(\|q\|^{2}+\delta_{3}\right) \delta_{4}}} \leq \mathcal{M}_{6}<\infty
$$

Similar we can prove that

$$
\frac{\|\hat{q}\|}{\sqrt{\delta_{2}^{2}+\left(\|\hat{q}\|^{2}+\delta_{3}\right) \delta_{4}}} \leq \mathcal{M}_{6}<\infty
$$


Now we take the limit of $T(\|q\|)$ as $\|q\| \longrightarrow 0$

$$
\lim _{\|q\| \longrightarrow 0} T(\|q\|)=\lim _{\|q\| \longrightarrow 0} \frac{\|q\|}{\sqrt{\delta_{2}^{2}+\left(\|q\|^{2}+\delta_{3}\right) \delta_{4}}}=\frac{0}{\sqrt{\delta_{2}^{2}+\left(0+\delta_{3}\right) \delta_{4}}}=0
$$

Thus the quantity $T(\|q\|)$ is not bounded below on $[0, \hat{\rho}]$. Note that the numerator and denominator of $T(\|q\|)$ is a continuous function on $[0, \tilde{\rho}]$. Moreover, by virtue of conditions $(i i)$ the denominator satisfies

$$
\sqrt{\delta_{2}^{2}+\delta_{3} \delta_{4}+\delta_{4}\|q\|} \geq \sqrt{\delta_{2}^{2}+\delta_{3} \delta_{4}}>0
$$

therefore, $T(\|q\|)$ ia a continuous nonnegative function on any closed interval that has a maximum $\mathcal{M}_{7}<\infty$. Denote by $\mathcal{M}_{8}=\operatorname{Max}\left\{\mathcal{M}_{6}, \mathcal{M}_{7}\right\}$ therefore,

$$
\begin{aligned}
& 0 \leq \frac{\|q\|+\|\hat{q}\|}{\sqrt{\delta_{2}^{2}+\left(\|q\|^{2}+\delta_{3}\right) \delta_{4}}+\sqrt{\delta_{2}^{2}+\left(\|\hat{q}\|^{2}+\delta_{3}\right) \delta_{4}}} \\
& \leq \frac{\mathcal{M}_{8} \sqrt{\delta_{2}^{2}+\left(\|q\|^{2}+\delta_{3}\right) \delta_{4}}+\mathcal{M}_{8} \sqrt{\delta_{2}^{2}+\left(\|\hat{q}\|^{2}+\delta_{3}\right) \delta_{4}}}{\sqrt{\delta_{2}^{2}+\left(\|q\|^{2}+\delta_{3}\right) \delta_{4}}+\sqrt{\delta_{2}^{2}+\left(\|\hat{q}\|^{2}+\delta_{3}\right) \delta_{4}}}=\mathcal{M}_{8}<\infty
\end{aligned}
$$

and the result follows.

\section{Lemma 5.2.5 Assume}

1. $q \in \mathbb{H}$.

2. $\delta_{4} \neq 0$ is a positive real number.

3. $\delta_{5} \neq 0$ and $\delta_{6} \neq 0$ are any real numbers and they have the same sign.

4. $\delta_{2}, \delta_{3}$, are any real numbers.

5. $\delta_{2}^{2}+\delta_{3} \delta_{4}>0$

then the quantity

$$
\left(\delta_{6}+\frac{\delta_{5}(\|q\|+\|\hat{q}\|)}{\|q\| \sqrt{\delta_{2}^{2}+\left(\|\hat{q}\|^{2}+\delta_{3}\right) \delta_{4}}+\|\hat{q}\| \sqrt{\delta_{2}^{2}+\left(\|q\|^{2}+\delta_{3}\right) \delta_{4}}}\right)^{2}
$$

is bounded above and below. 
Proof Clearly the quantity in (5.2.16) is bounded below

$$
0<\delta_{6}^{2} \leq\left(\delta_{6}+\frac{\delta_{5}(\|q\|+\|\hat{q}\|)}{\|q\| \sqrt{\delta_{2}^{2}+\left(\|\hat{q}\|^{2}+\delta_{3}\right) \delta_{4}}+\|\hat{q}\| \sqrt{\delta_{2}^{2}+\left(\|q\|^{2}+\delta_{3}\right) \delta_{4}}}\right)^{2}
$$

Next we will show that the quantity in (5.2.16) is bounded above. Since for all $0<\|q\|<\infty$ we have

$$
\sqrt{\delta_{2}^{2}+\left(\|q\|^{2}+\delta_{3}\right) \delta_{4}} \geq \sqrt{\delta_{2}^{2}+\delta_{3} \delta_{4}}>0
$$

and

$$
\sqrt{\delta_{2}^{2}+\left(\|\hat{q}\|^{2}+\delta_{3}\right) \delta_{4}} \geq \sqrt{\delta_{2}^{2}+\delta_{3} \delta_{4}}>0
$$

therefore,

$$
\begin{aligned}
& \left(\delta_{6}+\frac{\delta_{5}(\|q\|+\|\hat{q}\|)}{\|q\| \sqrt{\delta_{2}^{2}+\left(\|\hat{q}\|^{2}+\delta_{3}\right) \delta_{4}}+\|\hat{q}\| \sqrt{\delta_{2}^{2}+\left(\|q\|^{2}+\delta_{3}\right) \delta_{4}}}\right)^{2} \\
& \leq\left(\delta_{6}+\frac{\delta_{5}(\|q\|+\|\hat{q}\|)}{\|q\| \sqrt{\delta_{2}^{2}+\delta_{3} \delta_{4}}+\|\hat{q}\| \sqrt{\delta_{2}^{2}+\delta_{3} \delta_{4}}}\right)^{2} \\
& =\left(\delta_{6}+\frac{\delta_{5}(\|q\|+\|\hat{q}\|)}{\sqrt{\delta_{2}^{2}+\delta_{3} \delta_{4}}(\|q\|+\|\hat{q}\|)}\right)^{2}=\left(\delta_{6}+\frac{\delta_{5}}{\sqrt{\delta_{2}^{2}+\delta_{3} \delta_{4}}}\right)^{2}
\end{aligned}
$$

The proof is complete.

Lemma 5.2.6 Assume

1. $q \in \mathbb{H}$.

2. $\delta_{4} \neq 0$ is a positive real number.

3. $\delta_{5} \neq 0$ and $\delta_{6} \leq 0$ are any real numbers.

4. $\delta_{2}, \delta_{3}$, are any real numbers.

5. $\delta_{2}^{2}+\delta_{3} \delta_{4}>0$

6. $\delta_{6}+\frac{\delta_{5}}{\sqrt{\delta_{2}^{2}+\delta_{3} \delta_{4}}} \geq 0$ 
then the quantity

$$
\left(\delta_{6}+\frac{\delta_{5}(\|q\|+\|\hat{q}\|)}{\|q\| \sqrt{\delta_{2}^{2}+\left(\|\hat{q}\|^{2}+\delta_{3}\right) \delta_{4}}+\|\hat{q}\| \sqrt{\delta_{2}^{2}+\left(\|q\|^{2}+\delta_{3}\right) \delta_{4}}}\right)^{2}
$$

is bounded above but not bounded below.

Proof Assume that $\hat{q}=w q$ with $0<w \neq 1$ then we have

$$
\begin{aligned}
& \delta_{6}+\frac{\delta_{5}(\|q\|+w\|q\|)}{\|q\| \sqrt{\delta_{2}^{2}+\left(w^{2}\|q\|^{2}+\delta_{3}\right) \delta_{4}}+w\|q\| \sqrt{\delta_{2}^{2}+\left(\|q\|^{2}+\delta_{3}\right) \delta_{4}}} \\
& =\delta_{6}+\frac{\delta_{5}\|q\|(1+w)}{\|q\| \sqrt{\delta_{2}^{2}+\delta_{3} \delta_{4}+w\|q\|^{2} \delta_{4}}+w\|q\| \sqrt{\delta_{2}^{2}+\delta_{3} \delta_{4}+\|q\|^{2} \delta_{4}}} \\
& =\delta_{6}+\frac{\delta_{5}(1+w)}{\sqrt{\delta_{2}^{2}+\delta_{3} \delta_{4}+w\|q\|^{2} \delta_{4}}+w \sqrt{\delta_{2}^{2}+\delta_{3} \delta_{4}+\|q\|^{2} \delta_{4}}}
\end{aligned}
$$

Case1: $\delta_{6}=0$ since

$$
\lim _{\|q\| \rightarrow \infty} \delta_{6}+\frac{\delta_{5}(1+w)}{\sqrt{\delta_{2}^{2}+\delta_{3} \delta_{4}+w\|q\|^{2} \delta_{4}}+w \sqrt{\delta_{2}^{2}+\delta_{3} \delta_{4}+\|q\|^{2} \delta_{4}}}=0
$$

then (5.2.17) is not bounded below.

Case2: $\delta_{6}<0$ then

$$
\lim _{\|q\| \rightarrow \infty} \delta_{6}+\frac{\delta_{5}(1+w)}{\sqrt{\delta_{2}^{2}+\delta_{3} \delta_{4}+w\|q\|^{2} \delta_{4}}+w \sqrt{\delta_{2}^{2}+\delta_{3} \delta_{4}+\|q\|^{2} \delta_{4}}}=\delta_{6}<0
$$

Moreover

$$
\begin{aligned}
& \lim _{\|q\| \rightarrow 0} \delta_{6}+\frac{\delta_{5}(1+w)}{\sqrt{\delta_{2}^{2}+\delta_{3} \delta_{4}+w\|q\|^{2} \delta_{4}}+w \sqrt{\delta_{2}^{2}+\delta_{3} \delta_{4}+\|q\|^{2} \delta_{4}}} \\
& =\lim _{\|q\| \rightarrow 0} \delta_{6}+\frac{\delta_{5}(1+w)}{\sqrt{\delta_{2}^{2}+\delta_{3} \delta_{4}}+w \sqrt{\delta_{2}^{2}+\delta_{3} \delta_{4}}} \\
& =\lim _{\|q\| \rightarrow 0} \delta_{6}+\frac{\delta_{5}(1+w)}{\sqrt{\delta_{2}^{2}+\delta_{3} \delta_{4}}(1+w)}=\delta_{6}+\frac{\delta_{5}(1+w)}{\sqrt{\delta_{2}^{2}+\delta_{3} \delta_{4}}(1+w)}>0
\end{aligned}
$$

Clearly the quantity 5.2.17 is a continuous function on $(0, \infty)$. Since (5.2.18) and (5.2.19) have an opposite sign, then by the continuity there exists a 
finite value $0<v^{*}<\infty$ such that 5.2.17 is equal to zero, therefore it is not bounded below. Next we will show that the quantity 5.2.17 is bounded above. Use the fact that for any two real numbers $\rho_{1}$ and $\rho_{2}$ we have $\left(\rho_{1}+\rho_{2}\right)^{2} \leq$ $\left(\left|\rho_{1}\right|+\left|\rho_{2}\right|\right)^{2}$, thus

$$
\begin{aligned}
& \left(\delta_{6}+\frac{\delta_{5}(\|q\|+\|\hat{q}\|)}{\|q\| \sqrt{\delta_{2}^{2}+\left(\|\hat{q}\|^{2}+\delta_{3}\right) \delta_{4}}+\|\hat{q}\| \sqrt{\delta_{2}^{2}+\left(\|q\|^{2}+\delta_{3}\right) \delta_{4}}}\right)^{2} \\
& \leq\left(\left|\delta_{6}\right|+\frac{\left|\delta_{5}\right|(\|q\|+\|\hat{q}\|)}{\|q\| \sqrt{\delta_{2}^{2}+\left(\|\hat{q}\|^{2}+\delta_{3}\right) \delta_{4}}+\|\hat{q}\| \sqrt{\delta_{2}^{2}+\left(\|q\|^{2}+\delta_{3}\right) \delta_{4}}}\right)^{2}
\end{aligned}
$$

By the inequalities

$$
\sqrt{\delta_{2}^{2}+\left(\|q\|^{2}+\delta_{3}\right) \delta_{4}} \geq \sqrt{\delta_{2}^{2}+\delta_{3} \delta_{4}}>0
$$

and

$$
\sqrt{\delta_{2}^{2}+\left(\|\hat{q}\|^{2}+\delta_{3}\right) \delta_{4}} \geq \sqrt{\delta_{2}^{2}+\delta_{3} \delta_{4}}>0
$$

we have

$$
\begin{aligned}
& \left(\left|\delta_{6}\right|+\frac{\left|\delta_{5}\right|(\|q\|+\|\hat{q}\|)}{\|q\| \sqrt{\delta_{2}^{2}+\left(\|\hat{q}\|^{2}+\delta_{3}\right) \delta_{4}}+\|\hat{q}\| \sqrt{\delta_{2}^{2}+\left(\|q\|^{2}+\delta_{3}\right) \delta_{4}}}\right)^{2} \\
& \leq\left(\left|\delta_{6}\right|+\frac{\left|\delta_{5}\right|(\|q\|+\|\hat{q}\|)}{\|q\| \sqrt{\delta_{2}^{2}+\delta_{3} \delta_{4}}+\|\hat{q}\| \sqrt{\delta_{2}^{2}+\delta_{3} \delta_{4}}}\right)^{2} \\
& =\left(\left|\delta_{6}\right|+\frac{\left|\delta_{5}\right|(\|q\|+\|\hat{q}\|)}{\sqrt{\delta_{2}^{2}+\delta_{3} \delta_{4}}(\|q\|+\|\hat{q}\|)}\right)^{2}=\left(\left|\delta_{6}\right|+\frac{\left|\delta_{5}\right|}{\sqrt{\delta_{2}^{2}+\delta_{3} \delta_{4}}}\right)^{2} \leq 0
\end{aligned}
$$

The proof is complete.

\subsection{Equivalence Between An Extended Cone Metric and An Extended Paraboloid met- ric}

Another interesting property of the Ultra Extended Hilbert space there is an equivalence between an extended cone metric and an extended paraboloid metric. 
Theorem 5.3.1 Any extended cone metric is equivalent to any extended paraboloid metric.

Proof We proceed to prove theorem (5.3.1) by the following proposition and lemma.

Recall the formulas $t_{c}$ and $t_{p}$ in chapter two.

$$
t_{p}=\frac{-2 \alpha_{p}}{\mathcal{B}+\mathcal{B} \sqrt{G}}
$$

where

$$
\begin{gathered}
G=1-\frac{4\|q\|^{2} \alpha_{p}}{\mathcal{B}^{2}} \\
\mathcal{B}=2\left(\bar{\gamma}_{p}\left(\theta_{p}-y\right)+\gamma_{p} \overline{\left(\theta_{p}-y\right)}\right)
\end{gathered}
$$

and

$$
\alpha_{p}=-\mathcal{B}-4 y \bar{y}+2\left(y \bar{\theta}_{p}+\theta_{p} \bar{y}\right)
$$

Note that $\mathcal{B}>0$ and $\alpha_{p}<0$. And

$$
t_{c}=\frac{\alpha_{c}}{\|q\|+\frac{\left|\gamma_{c}\right|}{m}}
$$

where $\alpha_{c}=\frac{\left|\gamma_{c}\right|-\left|\theta_{c}\right|}{m}>0$

Proposition 5.3.2 Let $t_{p}$ and $t_{c}$ subject to (5.3.1) and (5.3.4) respectively then the quotient $\frac{t_{p}}{t_{c}}$ is bounded above and below.

Proof Compute the ratio between $t_{p}$ and $t_{c}$ as follows

$$
\frac{t_{p}}{t_{c}}=\frac{\frac{-2 \alpha_{p}}{\mathcal{B}+\mathcal{B} \sqrt{G}}}{\frac{\alpha_{c}}{\|q\|+\frac{\left|\gamma_{c}\right|}{m}}}=\frac{-2 \alpha_{p}\left(\|q\|+\frac{\left|\gamma_{c}\right|}{m}\right)}{\alpha_{c}(\mathcal{B}+\mathcal{B} \sqrt{G})}=\frac{-2 \alpha_{p}\left(\|q\|+\frac{\left|\gamma_{c}\right|}{m}\right)}{\alpha_{c}\left(\mathcal{B}+\mathcal{B} \sqrt{1-\frac{4\|q\|^{2} \alpha_{p}}{\mathcal{B}^{2}}}\right)}
$$

Take out $\frac{4\|q\|^{2} \alpha_{p}}{\mathcal{B}^{2}}$ from the square root to get

$$
\begin{aligned}
\frac{t_{p}}{t_{c}} & =\frac{-2 \alpha_{p}\|q\|\left(1+\frac{\left|\gamma_{c}\right|}{m\|q\|}\right)}{\alpha_{c} 2\|q\| \sqrt{-\alpha_{p}}\left(\frac{1}{2\|q\| \sqrt{-\alpha_{p}}}+\sqrt{\frac{-\mathcal{B}^{2}}{4\|q\|^{2} \alpha_{p}}+1}\right)} \\
& =\frac{\sqrt{-\alpha_{p}}\left(1+\frac{\left|\gamma_{c}\right|}{m\|q\|}\right)}{\alpha_{c}\left(\frac{1}{2\|q\| \sqrt{-\alpha_{p}}}+\sqrt{\frac{-\mathcal{B}^{2}}{4\|q\|^{2} \alpha_{p}}+1}\right)}
\end{aligned}
$$


Compute the limit as $\|q\| \longrightarrow \infty$

$$
\lim _{\|q\| \rightarrow \infty} \frac{t_{p}}{t_{c}}=\lim _{\|q\| \rightarrow \infty} \frac{\sqrt{-\alpha_{p}}\left(1+\frac{\left|\gamma_{c}\right|}{m\|q\|}\right)}{\alpha_{c}\left(\frac{1}{2\|q\| \sqrt{-\alpha_{p}}}+\sqrt{\frac{-\mathcal{B}^{2}}{4\|q\|^{2} \alpha_{p}}+1}\right)}=\frac{\sqrt{-\alpha_{p}}}{\alpha_{c}} \neq 0
$$

Thus the quantity $\frac{t_{p}}{t_{c}}$ is bounded above and below on $[\tilde{\rho}, \infty)$. Compute the limit as $\|q\| \longrightarrow 0$ to obtain

$$
\lim _{\|q\| \rightarrow 0} \frac{t_{p}}{t_{c}}=\lim _{\|q\| \rightarrow 0} \frac{-2 \alpha_{p}\left(\|q\|+\frac{\left|\gamma_{c}\right|}{m}\right)}{\alpha_{c}\left(\mathcal{B}+\mathcal{B} \sqrt{1-\frac{4\|q\|^{2} \alpha_{p}}{\mathcal{B}^{2}}}\right)}=\frac{-\alpha_{p}\left|\gamma_{c}\right|}{m \mathcal{B} \alpha_{c}} \neq 0
$$

Thus $\frac{t_{p}}{t_{c}}$ is bounded above and below on $[0, \hat{\rho}]$. Moreover, the denominator of $\frac{t_{p}}{t_{c}}$ does not equal zero for all $\|q\|$, assume that $0 \neq \hat{\rho} \leq\|q\| \leq \tilde{\rho} \neq \infty$ the quotient $\frac{t_{p}}{t_{c}}$ is a continuous function of $\|q\|$ on the closed interval $[\hat{\rho}, \tilde{\rho}]$. This implies that the quotient $\frac{t_{p}}{t_{c}}$ has a positive minimum and maximum on $[\hat{\rho}, \tilde{\rho}]$. The proof is complete.

Lemma 5.3.3 The ratio

$$
\frac{\left(\hat{t}_{p}-t_{p}\right)^{2} \gamma_{p} \bar{\gamma}_{p}+\left(t_{p}\|q\|-\hat{t}_{p}\|\hat{q}\|\right)^{2}}{\left(\hat{t}_{c}-t_{c}\right)^{2} \gamma_{c} \bar{\gamma}_{c}+\left(t_{c}\|q\|-\hat{t}_{c}\|\hat{q}\|\right)^{2}}
$$

is bounded above and below for all $\|q\|$ and $\|\hat{q}\|$.

Proof We proceed to show that there exist some real positive $\mathcal{N}_{5}, \mathcal{M}_{5}$ such that

$$
0<\mathcal{N}_{5} \leq \frac{\left(\hat{t}_{p}-t_{p}\right)^{2} \gamma_{p} \bar{\gamma}_{p}+\left(t_{p}\|q\|-\hat{t}_{p}\|\hat{q}\|\right)^{2}}{\left(\hat{t}_{c}-t_{c}\right)^{2} \gamma_{c} \bar{\gamma}_{c}+\left(t_{c}\|q\|-\hat{t}_{c}\|\hat{q}\|\right)^{2}} \leq \mathcal{M}_{5}<\infty
$$

Because both the numerator and denominator are zero when $\|q\|=\|\hat{q}\|$, so we have an indeterminate form. However we can factor the numerator and denominator to get $\|q\|-\|\hat{q}\|$ as a common factor that will be shown to cancel out. Recall the formulas (4.5.6) and (4.4.6) form chapter three

$$
\hat{t}_{p}-t_{p}=\frac{8 \alpha_{p}^{2}\left(\|q\|^{2}-\|\hat{q}\|^{2}\right)}{\mathcal{B}^{3}(1+\sqrt{\hat{G}})(1+\sqrt{G})(\sqrt{G}+\sqrt{\hat{G}})}
$$


and

$$
\hat{t}_{c}-t_{c}=\frac{\alpha_{c}(\|q\|-\|\hat{q}\|)}{\left(\|\hat{q}\|+\frac{\left|\gamma_{c}\right|}{m}\right)\left(\|q\|+\frac{\left|\gamma_{c}\right|}{m}\right)}
$$

Next we focus only on $\hat{t}_{c}\|\hat{q}\|-t_{c}\|q\|$ and $t_{p}\|q\|-\hat{t}_{p}\|\hat{q}\|$. The simpler conic related quantities.

$$
\begin{aligned}
t_{c}\|q\|-\hat{t}_{c}\|\hat{q}\| & =\frac{\alpha_{c}\|q\|}{\|q\|+\frac{\left|\gamma_{c}\right|}{m}}-\frac{\alpha_{c}\|\hat{q}\|}{\|\hat{q}\|+\frac{\left|\gamma_{c}\right|}{m}} \\
& =\frac{\alpha_{c}\|q\|\left(\|\hat{q}\|+\frac{\left|\gamma_{c}\right|}{m}\right)-\alpha_{c}\|\hat{q}\|\left(\|q\|+\frac{\left|\gamma_{c}\right|}{m}\right)}{\left(\|\hat{q}\|+\frac{\left|\gamma_{c}\right|}{m}\right)\left(\|q\|+\frac{\left|\gamma_{c}\right|}{m}\right)} \\
& =\frac{\alpha_{c}\|q\| \frac{\left|\gamma_{c}\right|}{m}-\alpha_{c}\|\hat{q}\| \frac{\left|\gamma_{c}\right|}{m}}{\left(\|\hat{q}\|+\frac{\left|\gamma_{c}\right|}{m}\right)\left(\|q\|+\frac{\left|\gamma_{c}\right|}{m}\right)}=\frac{\left|\gamma_{c}\right|}{m} \frac{\alpha_{c}(\|q\|-\|\hat{q}\|)}{\left(\|\hat{q}\|+\frac{\left|\gamma_{c}\right|}{m}\right)\left(\|q\|+\frac{\left|\gamma_{c}\right|}{m}\right)} \\
& =\frac{\left|\gamma_{c}\right|}{m}\left(\hat{t}_{c}-t_{c}\right)
\end{aligned}
$$

We now calculate the paraboloid related quantities.

$$
\begin{aligned}
t_{p}\|q\|-\hat{t}_{p}\|\hat{q}\| & =\frac{-2 \alpha_{p}\|q\|}{\mathcal{B}+\mathcal{B} \sqrt{G}-\frac{-2 \alpha_{p}\|\hat{q}\|}{\mathcal{B}+\mathcal{B} \sqrt{\hat{G}}}} \\
& =\frac{-2 \alpha_{p}(\|q\|(\mathcal{B}+\mathcal{B} \sqrt{\hat{G}})-\|\hat{q}\|(\mathcal{B}+\mathcal{B} \sqrt{G}))}{(\mathcal{B}+\mathcal{B} \sqrt{G})(\mathcal{B}+\mathcal{B} \sqrt{\hat{G}})} \\
& =\frac{-2 \alpha_{p}(\|q\|(1+\sqrt{\hat{G}})-\|\hat{q}\|(1+\sqrt{G}))}{\mathcal{B}(1+\sqrt{G})(1+\sqrt{\hat{G}})} \\
& =\frac{-2 \alpha_{p}(\|q\|-\|\hat{q}\|+\|q\| \sqrt{\hat{G}}-\|\hat{q}\| \sqrt{G})}{\mathcal{B}(1+\sqrt{G})(1+\sqrt{\hat{G}})} \\
& =\frac{-2 \alpha_{p}(\|q\|-\|\hat{q}\|)}{\mathcal{B}(1+\sqrt{G})(1+\sqrt{\hat{G}})}+\frac{-2 \alpha_{p}(\|q\| \sqrt{\hat{G}}-\|\hat{q}\| \sqrt{G})}{\mathcal{B}(1+\sqrt{G})(1+\sqrt{\hat{G}})}
\end{aligned}
$$


multiply by the conjugate to obtain

$$
\begin{aligned}
& t_{p}\|q\|-\hat{t}_{p}\|\hat{q}\| \frac{-2 \alpha_{p}(\|q\|-\|\hat{q}\|)}{\mathcal{B}(1+\sqrt{G})(1+\sqrt{\hat{G}})}+\frac{-2 \alpha_{p}\left(\|q\|^{2} \hat{G}-\|\hat{q}\|^{2} G\right)}{\mathcal{B}(1+\sqrt{G})(1+\sqrt{\hat{G}})(\|q\| \sqrt{\hat{G}}+\|\hat{q}\| \sqrt{G})} \\
&= \frac{-2 \alpha_{p}(\|q\|-\|\hat{q}\|)}{\mathcal{B}(1+\sqrt{G})(1+\sqrt{\hat{G}})}+\frac{-2 \alpha_{p}\left(\|q\|^{2}\left(1-\frac{4\|\hat{q}\|^{2} \alpha_{p}}{\mathcal{B}^{2}}\right)-\|\hat{q}\|^{2}\left(1-\frac{4\|q\|^{2} \alpha_{p}}{\mathcal{B}^{2}}\right)\right)}{\mathcal{B}(1+\sqrt{G})(1+\sqrt{\hat{G}})(\|q\| \sqrt{\hat{G}}+\|\hat{q}\| \sqrt{G})} \\
&= \frac{-2 \alpha_{p}(\|q\|-\|\hat{q}\|)}{\mathcal{B}(1+\sqrt{G})(1+\sqrt{\hat{G}})}+\frac{-2 \alpha_{p}\left(\|q\|^{2}-\|\hat{q}\|^{2}\right)}{\mathcal{B}(1+\sqrt{G})(1+\sqrt{\hat{G}})(\|q\| \sqrt{\hat{G}}+\|\hat{q}\| \sqrt{G})} \\
&= \frac{-2 \alpha_{p}(\|q\|-\|\hat{q}\|)}{\mathcal{B}(1+\sqrt{G})(1+\sqrt{\hat{G}})}+\frac{-\|q\|-\|\hat{q}\|)(\|q\|+\|\hat{q}\|)}{\mathcal{B}(1+\sqrt{G})(1+\sqrt{\hat{G}})(\|q\| \sqrt{\hat{G}}+\|\hat{q}\| \sqrt{G})} \\
& \quad=\frac{-2 \alpha_{p}(\|q\|-\|\hat{q}\|)(\|q\| \sqrt{\hat{G}}+\|\hat{q}\| \sqrt{G}+\|q\|+\|\hat{q}\|)}{\mathcal{B}(1+\sqrt{G})(1+\sqrt{\hat{G}})(\|q\| \sqrt{\hat{G}}+\|\hat{q}\| \sqrt{G})} \quad(5.3 .12)
\end{aligned}
$$

therefore

$$
\begin{aligned}
& \frac{\left(\hat{t}_{p}-t_{p}\right)^{2} \gamma_{p} \bar{\gamma}_{p}+\left(t_{p}\|q\|-\hat{t}_{p}\|\hat{q}\|\right)^{2}}{\left(\hat{t}_{c}-t_{c}\right)^{2} \gamma_{c} \bar{\gamma}_{c}+\left(t_{c}\|q\|-\hat{t}_{c}\|\hat{q}\|\right)^{2}}=\frac{\left(\hat{t}_{p}-t_{p}\right)^{2} \gamma_{p} \bar{\gamma}_{p}+\left(t_{p}\|q\|-\hat{t}_{p}\|\hat{q}\|\right)^{2}}{\left(\hat{t}_{c}-t_{c}\right)^{2} \gamma_{c} \bar{\gamma}_{c}+\frac{\left|\gamma_{c}\right|^{2}}{m^{2}}\left(\hat{t}_{c}-t_{c}\right)^{2}} \\
& =\frac{\left(\hat{t}_{p}-t_{p}\right)^{2} \gamma_{p} \bar{\gamma}_{p}+\left(t_{p}\|q\|-\hat{t}_{p}\|\hat{q}\|\right)^{2}}{\left(\hat{t}_{c}-t_{c}\right)^{2}\left(\gamma_{c} \bar{\gamma}_{c}+\frac{\left|\gamma_{c}\right|^{2}}{m^{2}}\right)}=\frac{\left(\hat{t}_{p}-t_{p}\right)^{2} \gamma_{p} \bar{\gamma}_{p}+\left(t_{p}\|q\|-\hat{t}_{p}\|\hat{q}\|\right)^{2}}{\left(\frac{\alpha_{c}(\|q\|-\|\hat{q}\|)}{\left(\|\hat{q}\|+\frac{\left|\gamma_{c}\right|}{m}\right)\left(\|q\|+\frac{\left|\gamma_{c}\right|}{m}\right)}\right)^{2}\left(\gamma_{c} \bar{\gamma}_{c}+\frac{\left|\gamma_{c}\right|^{2}}{m^{2}}\right)} \\
& =\frac{\left(\frac{8 \alpha_{p}^{2}\left(\|q\|^{2}-\|\hat{q}\|^{2}\right)}{\mathcal{B}^{3}(1+\sqrt{\hat{G}})(1+\sqrt{G})(\sqrt{G}+\sqrt{\hat{G}})}\right)^{2} \gamma_{p} \bar{\gamma}_{p}}{\left(\frac{\alpha_{c}(\|q\|-\|\hat{q}\|)}{\left(\|\hat{q}\|+\frac{\left|\gamma_{c}\right|}{m}\right)\left(\|q\|+\frac{\left|\gamma_{c}\right|}{m}\right)}\right)^{2}\left(\gamma_{c} \bar{\gamma}_{c}+\frac{\left|\gamma_{c}\right|^{2}}{m^{2}}\right)} \\
& +\frac{\left(\frac{-2 \alpha_{p}(\|q\|-\|\hat{q}\|)(\|q\| \sqrt{\hat{G}}+\|\hat{q}\| \sqrt{G}+\|q\|+\|\hat{q}\|)}{\mathcal{B}(1+\sqrt{G})(1+\sqrt{\hat{G}})(\|q\| \sqrt{\hat{G}}+\|\hat{q}\| \sqrt{G})}\right)^{2}}{\left(\frac{\alpha_{c}(\|q\|-\|\hat{q}\|)}{\left(\|\hat{q}\|+\frac{|\gamma c|}{m}\right)\left(\|q\|+\frac{\left|\gamma_{c}\right|}{m}\right)}\right)^{2}\left(\gamma_{c} \bar{\gamma}_{c}+\frac{\left|\gamma_{c}\right|^{2}}{m^{2}}\right)}
\end{aligned}
$$




$$
\begin{gathered}
=\frac{\left(\frac{8 \alpha_{p}^{2}(\|q\|+\|\hat{\hat{q}}\|)}{\mathcal{B}^{3}(1+\sqrt{\hat{G}})(1+\sqrt{G})(\sqrt{G}+\sqrt{\hat{G}})}\right)^{2} \gamma_{p} \bar{\gamma}_{p}}{\left(\frac{\alpha_{c}}{\left(\|\hat{q}\|+\frac{\left|\gamma_{c}\right|}{m}\right)\left(\|q\|+\frac{\left|\gamma_{c}\right|}{m}\right)}\right)^{2}\left(\gamma_{c} \bar{\gamma}_{c}+\frac{\left|\gamma_{c}\right|^{2}}{m^{2}}\right)} \\
+\frac{\left(\frac{-2 \alpha_{p}(\|q\| \sqrt{\hat{G}}+\|\hat{q}\| \sqrt{G}+\|q\|+\|\hat{q}\|)}{\mathcal{B}(1+\sqrt{G})(1+\sqrt{\hat{G}})(\|q\| \sqrt{\hat{G}}+\|\hat{q}\| \sqrt{G})}\right)^{2}}{\left(\frac{\alpha_{c}}{\left(\|\hat{q}\|+\frac{\left|\gamma_{c}\right|}{m}\right)\left(\|q\|+\frac{\left|\gamma_{c}\right|}{m}\right)}\right)^{2}\left(\gamma_{c} \bar{\gamma}_{c}+\frac{\left|\gamma_{c}\right|^{2}}{m^{2}}\right)} \\
=\frac{\left(\|\hat{q}\|+\frac{\left|\gamma_{c}\right|}{m}\right)^{2}\left(\|q\|+\frac{\left|\gamma_{c}\right|}{m}\right)^{2}\left(8 \alpha_{p}^{2}(\|q\|+\|\hat{q}\|)\right)^{2} \gamma_{p} \bar{\gamma}_{p}}{\left(\alpha_{c} \mathcal{B}^{3}(1+\sqrt{\hat{G}})(1+\sqrt{G})(\sqrt{G}+\sqrt{\hat{G}})\right)^{2}\left(\gamma_{c} \bar{\gamma}_{c}+\frac{\left|\gamma_{c}\right|^{2}}{m^{2}}\right)} \\
+\frac{\left.\mid\|\hat{q}\|+\frac{\left|\gamma_{c}\right|}{m}\right)^{2}\left(\|q\|+\frac{\left|\gamma_{c}\right|}{m}\right)^{2}\left(-2 \alpha_{p}(\|q\| \sqrt{\hat{G}}+\|\hat{q}\| \sqrt{G}+\|q\|+\|\hat{q}\|)\right)^{2}}{\left(\alpha_{c} \mathcal{B}(1+\sqrt{G})(1+\sqrt{\hat{G}})(\|q\| \sqrt{\hat{G}}+\|\hat{q}\| \sqrt{G})\right)^{2}\left(\gamma_{c} \bar{\gamma}_{c}+\frac{\left|\gamma_{c}\right|^{2}}{m^{2}}\right)}
\end{gathered}
$$

We could now apply lemmas 5.2.3, 5.2.4 and 5.2.5. Since $G=1-\frac{4\|q\|^{2} \alpha_{p}}{\mathcal{B}^{2}}$ and $\hat{G}=1-\frac{4\|\hat{q}\|^{2} \alpha_{p}}{\mathcal{B}^{2}}$, note that $\delta_{1}=\frac{\left|\gamma_{c}\right|}{m}>0, \delta_{2}=1 \neq 0, \delta_{3}=0, \delta_{4}=-\frac{4 \alpha_{p}}{\mathcal{B}^{2}}>0$, $\delta_{5}=1>0$ and $\delta_{6}=-2 \alpha_{p} \neq 0$. Also we can note that $\delta_{2}^{2}+\delta_{3} \delta_{4}>0$ and $\sqrt{\delta_{2}^{2}+\delta_{3} \delta_{4}}+\delta_{2}>0$. Thus

$$
\frac{\left(\hat{t}_{p}-t_{p}\right)^{2} \gamma_{p} \bar{\gamma}_{p}+\left(t_{p}\|q\|-\hat{t}_{p}\|\hat{q}\|\right)^{2}}{\left(\hat{t}_{c}-t_{c}\right)^{2} \gamma_{c} \bar{\gamma}_{c}+\left(t_{c}\|q\|-\hat{t}_{c}\|\hat{q}\|\right)^{2}}
$$

is bounded above and below. The proof is complete.

The conclusion proof of theorem (5.3.1) as following. By proposition 5.3.2, lemma 5.3.3 and theorem 5.2.2 the result follows.

\subsection{Equivalence Between Two Extended Cone Metrics}

Our goal is to show the equivalence between two extended cone metrics .

Theorem 5.4.1 Any two distinct extended cone metrics are equivalent. 
Proof We proceed to prove theorem (5.4.1) by the following proposition.

Proposition 5.4.2 Assume

$$
t_{c_{1}}=\frac{\alpha_{c_{1}}}{\|q\|+\frac{\left|\gamma_{c_{1}}\right|}{m}} \quad, \hat{t}_{c_{1}}=\frac{\alpha_{c_{1}}}{\|\hat{q}\|+\frac{\left|\gamma_{c_{1}}\right|}{m}}
$$

and

$$
t_{c_{1}}=\frac{\alpha_{c_{2}}}{\|q\|+\frac{\mid \gamma_{c_{2} \mid}}{m}} \quad, \hat{t}_{c_{2}}=\frac{\alpha_{c_{2}}}{\|\hat{q}\|+\frac{\left|\gamma_{c_{2}}\right|}{m}}
$$

then the quantities

$$
\begin{gathered}
\left|\frac{\hat{t}_{c_{1}}-t_{c_{1}}}{\hat{t}_{c_{2}}-t_{c_{2}}}\right| \\
\left|\frac{\hat{t}_{c_{1}}\|\hat{q}\|-t_{c_{1}}\|q\|}{\hat{t}_{c_{2}}\|\hat{q}\|-t_{c_{2}}\|q\|}\right|
\end{gathered}
$$

are bounded above and below.

Proof It is enough to show that the quantity $\frac{\hat{t}_{c_{1}}-t_{c_{1}}}{\hat{t}_{c_{2}}-t_{c_{2}}}$ is bounded above and below, this is because $\hat{t}_{c}\|\hat{q}\|-t_{c}\|q\|=\frac{\left|\gamma_{c}\right|}{m}\left(\hat{t}_{c}-t_{c}\right)$. The first step is find the ratio $\frac{\hat{t}_{c_{1}}-t_{c_{1}}}{\hat{t}_{c_{2}}-t_{c_{2}}}$

$$
\begin{aligned}
\frac{\hat{t}_{c_{1}}-t_{c_{1}}}{\hat{t}_{c_{2}}-t_{c_{2}}}= & \frac{\frac{\alpha_{c_{1}}}{\|\hat{q}\|+\frac{\left|\gamma_{c_{1}}\right|}{m}}-\frac{\alpha_{c_{1}}}{\|q\|+\frac{\left|\gamma_{c_{1}}\right|}{m}}}{\frac{\alpha_{c_{2}}}{\|\hat{q}\|+\frac{\left|\gamma_{c_{2}}\right|}{m}}-\frac{\alpha_{c_{2}}}{\|q\|+\frac{\left|\gamma_{c_{2}}\right|}{m}}} \\
= & \frac{\alpha_{c_{1}}(\|q\|-\|\hat{q}\|)\left(\|\hat{q}\|+\frac{\left|\gamma_{c_{2}}\right|}{m}\right)\left(\|q\|+\frac{\left|\gamma_{c_{2}}\right|}{m}\right)}{\alpha_{c_{2}}(\|q\|-\|\hat{q}\|)\left(\|\hat{q}\|+\frac{\left|\gamma_{c_{1}}\right|}{m}\right)\left(\|q\|+\frac{\left|\gamma_{c_{1}}\right|}{m}\right)} \\
= & \frac{\alpha_{c_{1}}\left(\|\hat{q}\|+\frac{\left|\gamma_{c_{2}}\right|}{m}\right)\left(\|q\|+\frac{\left|\gamma_{c_{2}}\right|}{m}\right)}{\alpha_{c_{2}}\left(\|\hat{q}\|+\frac{\left|\gamma_{c_{1}}\right|}{m}\right)\left(\|q\|+\frac{\left|\gamma_{c_{1}}\right|}{m}\right)} \\
= & \frac{\alpha_{c_{1}}\left(1+\frac{\left|\gamma_{c_{2}}\right|}{m\|\hat{q}\|}\right)\left(1+\frac{\left|\gamma_{c_{2}}\right|}{m\|q\|}\right)}{\alpha_{c_{2}}\left(1+\frac{\left|\gamma_{c_{1}}\right|}{m\|\hat{q}\|}\right)\left(1+\frac{\left|\gamma_{c_{1}}\right|}{m\|q\|}\right)}
\end{aligned}
$$


The denominator of $\frac{\hat{t}_{c_{1}}-t_{c_{1}}}{\hat{t}_{c_{2}}-t_{c_{2}}}$ does not equal zero for all $\|q\|$ and $\|\hat{q}\|$. Compute the limit of the quantity $\frac{\hat{t}_{c_{1}}-t_{c_{1}}}{\hat{t}_{c_{2}}-t_{c_{2}}}$ as $\|q\| \longrightarrow 0$ and as $\|\hat{q}\| \longrightarrow 0$

$$
\begin{aligned}
\lim _{\substack{\|q\| \rightarrow 0 \\
\|\hat{q}\| \longrightarrow 0}} \frac{\hat{t}_{c_{1}}-t_{c_{1}}}{\hat{t}_{c_{2}}-t_{c_{2}}}= & \lim _{\substack{\|q\| \rightarrow 0 \\
\|\hat{q}\| \longrightarrow 0}} \frac{\alpha_{c_{1}}\left(\|\hat{q}\|+\frac{\left|\gamma_{c_{2}}\right|}{m}\right)\left(\|q\|+\frac{\left|\gamma_{c_{2}}\right|}{m}\right)}{\alpha_{c_{2}}\left(\|\hat{q}\|+\frac{\left|\gamma_{c_{1}}\right|}{m}\right)\left(\|q\|+\frac{\left|\gamma_{c_{1}}\right|}{m}\right)} \\
& =\frac{\alpha_{c_{1}}\left(0+\frac{\left|\gamma_{c_{2}}\right|}{m}\right)\left(0+\frac{\left|\gamma_{c_{2}}\right|}{m}\right)}{\alpha_{c_{2}}\left(0+\frac{\left|\gamma_{c_{1}}\right|}{m}\right)\left(0+\frac{\left|\gamma_{c_{1}}\right|}{m}\right)}=\frac{\alpha_{c_{1}}}{\alpha_{c_{2}}} \neq 0
\end{aligned}
$$

Thus the quantity

$$
\left|\frac{\hat{t}_{c_{1}}-t_{c_{1}}}{\hat{t}_{c_{2}}-t_{c_{2}}}\right|
$$

is bounded above and below. Compute the limit of the quantity $\frac{\hat{t}_{c_{1}}-t_{c_{1}}}{\hat{t}_{c_{2}}-t_{c_{2}}}$ as $\|q\| \longrightarrow \infty$ and as $\|\hat{q}\| \longrightarrow \infty$.

$$
\begin{aligned}
\lim _{\substack{\|q\| \longrightarrow \infty \\
\|\hat{q}\| \longrightarrow \infty}} \frac{\hat{t}_{c_{1}}-t_{c_{1}}}{\hat{t}_{c_{2}}-t_{c_{2}}} & =\lim _{\substack{\|q\| \longrightarrow \infty \\
\|\hat{q}\| \longrightarrow \infty}} \frac{\alpha_{c_{1}}\left(1+\frac{\left|\gamma_{c_{2}}\right|}{m\|\hat{q}\|}\right)\left(1+\frac{\left|\gamma_{c_{2}}\right|}{m\|q\|}\right)}{\alpha_{c_{2}}\left(1+\frac{\left|\gamma_{c_{1}}\right|}{m\|\hat{q}\|}\right)\left(1+\frac{\left|\gamma_{c_{1}}\right|}{m\|q\|}\right)} \\
& =\frac{\alpha_{c_{1}}(1+0)(1+0)}{\alpha_{c_{2}}(1+0)(1+0)}=\frac{\alpha_{c_{1}}}{\alpha_{c_{2}}} \neq 0
\end{aligned}
$$

Thus the quantity

$$
\left|\frac{\hat{t}_{c_{1}}-t_{c_{1}}}{\hat{t}_{c_{2}}-t_{c_{2}}}\right|
$$

is bounded above and below. If $0 \neq \rho_{1} \leq\|q\| \leq \rho_{2} \neq \infty$ and $0 \neq \hat{\rho}_{1} \leq\|\hat{q}\| \leq$ $\hat{\rho}_{2} \neq \infty$ then we have

$$
\begin{aligned}
\left|\frac{\hat{t}_{c_{1}}-t_{c_{1}}}{\hat{t}_{c_{2}}-t_{c_{2}}}\right| & =\frac{\alpha_{c_{1}}\left(\|\hat{q}\|+\frac{\left|\gamma_{c_{2}}\right|}{m}\right)\left(\|q\|+\frac{\left|\gamma_{c_{2}}\right|}{m}\right)}{\alpha_{c_{2}}\left(\|\hat{q}\|+\frac{\left|\gamma_{c_{1}}\right|}{m}\right)\left(\|q\|+\frac{\left|\gamma_{c_{1}}\right|}{m}\right)} \\
& \leq \frac{\alpha_{c_{1}}\left(\left\|\hat{\rho}_{2}\right\|+\frac{\left|\gamma_{c_{2}}\right|}{m}\right)\left(\left\|\rho_{2}\right\|+\frac{\left|\gamma_{c_{2}}\right|}{m}\right)}{\alpha_{c_{2}}\left(\left\|\hat{\rho}_{1}\right\|+\frac{\left|\gamma_{c_{1}}\right|}{m}\right)\left(\left\|\rho_{1}\right\|+\frac{\left|\gamma_{c_{1}}\right|}{m}\right)} \neq \infty
\end{aligned}
$$


and

$$
\begin{aligned}
\left|\frac{\hat{t}_{c_{1}}-t_{c_{1}}}{\hat{t}_{c_{2}}-t_{c_{2}}}\right| & =\frac{\alpha_{c_{1}}\left(\|\hat{q}\|+\frac{\left|\gamma_{c_{2}}\right|}{m}\right)\left(\|q\|+\frac{\left|\gamma_{c_{2}}\right|}{m}\right)}{\alpha_{c_{2}}\left(\|\hat{q}\|+\frac{\left|\gamma_{c_{1}}\right|}{m}\right)\left(\|q\|+\frac{\left|\gamma_{c_{1}}\right|}{m}\right)} \\
& \geq \frac{\alpha_{c_{1}}\left(\left\|\hat{\rho}_{1}\right\|+\frac{\left|\gamma_{c_{2}}\right|}{m}\right)\left(\left\|\rho_{1}\right\|+\frac{\left|\gamma_{c_{2}}\right|}{m}\right)}{\alpha_{c_{2}}\left(\left\|\hat{\rho}_{2}\right\|+\frac{\left|\gamma_{c_{1}}\right|}{m}\right)\left(\left\|\rho_{2}\right\|+\frac{\left|\gamma_{c_{1}}\right|}{m}\right)}
\end{aligned}
$$

therefore $\left|\frac{\hat{t}_{c_{1}}-t_{c_{1}}}{\hat{t}_{c_{2}}-t_{c_{2}}}\right|$ it is bounded above and below. Since

$$
\frac{\hat{t}_{c_{1}}\|\hat{q}\|-t_{c_{1}}\|q\|}{\hat{t}_{c_{2}}\|\hat{q}\|-t_{c_{2}}\|q\|}=\frac{\frac{\left|\gamma_{c_{1}}\right|}{m}\left(\hat{t}_{c_{1}}-t_{c_{1}}\right)}{\frac{\left|\gamma_{c_{2}}\right|}{m}\left(\hat{t}_{c_{2}}-t_{c_{2}}\right)}
$$

then the quantity

$$
\left|\frac{\hat{t}_{c_{1}}\|\hat{q}\|-t_{c_{1}}\|q\|}{\hat{t}_{c_{2}}\|\hat{q}\|-t_{c_{2}}\|q\|}\right|
$$

is bounded above and below. The proof is complete.

The conclusion proof of theorem (5.4.1) as following. By the theorem 5.2.1 and the proposition 5.4.2 the result follow.

Corollary 5.4.3 Any two extended paraboloid metrics are equivalent.

Proof Consider any two extended paraboloid metrics, then by theorems 5.3.1 and 5.4.1 the result follow.

\subsection{Equivalence Between An Extended Cone Metric and An Extended Hyperboloid Met- ric}

Our goal is to show the equivalence between any extended cone metric and any extended hyperboloid metric.

Theorem 5.5.1 Any extended cone metric is equivalent to any extended hyperboloid metric. 
Proof We proceed to prove theorem (5.5.1) by the following of proposition and lemma.

Recall the formula $t_{h}$ in chapter two.

$$
t_{h}=\frac{-\alpha_{h}}{\mathcal{C}+\sqrt{E}}
$$

where

$$
E=\mathcal{C}^{2}-\left(\|q\|^{2}-\mathcal{C}\right) \alpha_{h}
$$

$\mathcal{C}=\left(\frac{\operatorname{Re}\left(\theta_{h} \bar{\gamma}_{h}\right)}{b}\right)^{2}-\gamma_{h} \bar{\gamma}_{h}$ and $\alpha_{h}=-\mathcal{C}+\theta_{h} \bar{\theta}_{h}-b^{2}$. Note that $\mathcal{C}>0$ and $\alpha_{h}<0$

Proposition 5.5.2 Let $t_{h}$ and $t_{c}$ subject to (5.5.1) and (5.3.4) respectively then we the quantity $\frac{t_{h}}{t_{c}}$ is bounded above and below.

Proof First we the ratio between $t_{h}$ and $t_{c}$

$$
\frac{t_{h}}{t_{c}}=\frac{\frac{-\alpha_{h}}{\mathcal{C}+\sqrt{E}}}{\frac{\alpha_{c}}{\|q\|+\frac{\left|\gamma_{c}\right|}{m}}}=\frac{-\alpha_{h}\left(\|q\|+\frac{\left|\gamma_{c}\right|}{m}\right)}{\alpha_{c}\left(\mathcal{C}+\sqrt{\mathcal{C}^{2}-\left(\|q\|^{2}-\mathcal{C}\right) \alpha_{h}}\right)}
$$

Take out $\|q\|^{2}$ from the square root to get

$$
\begin{gathered}
\frac{t_{h}}{t_{c}}=\frac{-\alpha_{h}\|q\|\left(1+\frac{\left|\gamma_{c}\right|}{m\|q\|}\right)}{\alpha_{c}\|q\|\left(\frac{\mathcal{C}}{\|q\|}+\sqrt{\frac{\mathcal{C}^{2}}{\|q\|^{2}}-\left(1-\frac{\mathcal{C}}{\|q\|^{2}}\right) \alpha_{h}}\right)} \\
=\frac{-\alpha_{h}\left(1+\frac{\left|\gamma_{c}\right|}{m\|q\|}\right)}{\alpha_{c}\left(\frac{\mathcal{C}}{\|q\|}+\sqrt{\frac{\mathcal{C}^{2}}{\|q\|^{2}}-\left(1-\frac{\mathcal{C}}{\|q\|^{2}}\right) \alpha_{h}}\right)}
\end{gathered}
$$

Compute the limit of the ratio $\frac{t_{h}}{t_{c}}$ as $\|q\| \longrightarrow 0$ and as $\|q\| \longrightarrow 0$.

$$
\begin{aligned}
\lim _{\|q\| \rightarrow 0} \frac{t_{h}}{t_{c}} & =\lim _{\|q\| \rightarrow 0} \frac{-\alpha_{h}\left(\|q\|+\frac{\left|\gamma_{c}\right|}{m}\right)}{\alpha_{c}\left(\mathcal{C}+\sqrt{\mathcal{C}^{2}-\left(\|q\|^{2}-\mathcal{C}\right) \alpha_{h}}\right)} \\
& =\frac{-\alpha_{h} \frac{\left|\gamma_{c}\right|}{m}}{\alpha_{c}\left(\mathcal{C}+\sqrt{\mathcal{C}^{2}+\mathcal{C} \alpha_{h}}\right)} \neq 0
\end{aligned}
$$


then the quotient $\frac{t_{h}}{t_{c}}$ is bounded above and below on $[0, \hat{\rho}]$. Compute the limit of the ratio $\frac{t_{h}}{t_{c}}$ as $\|q\| \longrightarrow 0$ and as $\|q\| \longrightarrow 0$.

$$
\begin{aligned}
\lim _{\|q\| \rightarrow \infty} \frac{t_{h}}{t_{c}} & =\lim _{\|q\| \longrightarrow \infty} \frac{-\alpha_{h}\left(1+\frac{\left|\gamma_{c}\right|}{m\|q\|}\right)}{\alpha_{c}\left(\frac{\mathcal{C}}{\|q\|}+\sqrt{\frac{\mathcal{C}^{2}}{\|q\|^{2}}-\left(1-\frac{\mathcal{C}}{\|q\|^{2}}\right) \alpha_{h}}\right)} \\
& =\frac{-\alpha_{h}}{\alpha_{c} \sqrt{-\alpha_{h}}}=\frac{\sqrt{-\alpha_{h}}}{\alpha_{c}} \neq 0
\end{aligned}
$$

then $\frac{t_{h}}{t_{c}}$ is bounded above and below on $[\tilde{\rho}, \infty)$. Moreover, the denominator of $\frac{t_{h}}{t_{c}}$ does not equal zero for all $\|q\|$, if $0 \neq \hat{\rho} \leq\|q\| \leq \tilde{\rho} \neq \infty$ then the quotient $\frac{t_{h}}{t_{c}}$ is a continuous function of $\|q\|$ on the closed interval $[\hat{\rho}, \tilde{\rho}]$. This implies that the quotient $\frac{t_{h}}{t_{c}}$ has a positive minimum and maximum on $[\hat{\rho}, \tilde{\rho}]$. The result follow.

Lemma 5.5.3 The ratio

$$
\frac{\left(\hat{t}_{h}-t_{h}\right)^{2} \gamma_{h} \bar{\gamma}_{h}+\left(t_{h}\|q\|-\hat{t}_{h}\|\hat{q}\|\right)^{2}}{\left(\hat{t}_{c}-t_{c}\right)^{2} \gamma_{c} \bar{\gamma}_{c}+\left(t_{c}\|q\|-\hat{t}_{c}\|\hat{q}\|\right)^{2}}
$$

is bounded above and below for all $\|q\|$ and $\|\hat{q}\|$.

Proof We factor the numerator and denominator to get $\|q\|-\|\hat{q}\|$ as a common factor that will be shown to cancel out. Recall the formula (4.6.6) form chapter three

$$
\hat{t}_{h}-t_{h}=\frac{\alpha_{h}^{2}\left(\|q\|^{2}-\|\hat{q}\|^{2}\right)}{(\mathcal{C}+\sqrt{\hat{E}})(\mathcal{C}+\sqrt{E})(\sqrt{E}+\sqrt{\hat{E}})}
$$

We now calculate the hyperboloid related quantities.

$$
\begin{aligned}
t_{h}\|q\|-\hat{t}_{h}\|\hat{q}\| & =\frac{-\alpha_{h}\|q\|}{\mathcal{C}+\sqrt{E}}-\frac{-\alpha_{h}\|\hat{q}\|}{\mathcal{C}+\sqrt{\hat{E}}} \\
& =\frac{-\alpha_{h}(\mathcal{C}(\|q\|-\|\hat{q}\|)+\|q\| \sqrt{\hat{E}}-\|\hat{q}\| \sqrt{E})}{(\mathcal{C}+\sqrt{E})(\mathcal{C}+\sqrt{\hat{E}})}
\end{aligned}
$$


multiply by the conjugate to obtain

$$
\begin{aligned}
& t_{h}\|q\|-\hat{t}_{h}\|\hat{q}\| \\
& =\frac{-\alpha_{h}\left(\mathcal{C}(\|q\|-\|\hat{q}\|)(\|q\| \sqrt{\hat{E}}+\|\hat{q}\| \sqrt{E})+\|q\|^{2} \hat{E}-\|\hat{q}\|^{2} E\right)}{(\mathcal{C}+\sqrt{E})(\mathcal{C}+\sqrt{\hat{E}})(\|q\| \sqrt{\hat{E}}+\|\hat{q}\| \sqrt{E})} \\
& =\frac{-\alpha_{h}\left(\mathcal{C}(\|q\|-\|\hat{q}\|)(\|q\| \sqrt{\hat{E}}+\|\hat{q}\| \sqrt{E})+\left(\mathcal{C}^{2}+\mathcal{C} \alpha_{h}\right)\left(\|q\|^{2}-\|\hat{q}\|^{2}\right)\right)}{(\mathcal{C}+\sqrt{E})(\mathcal{C}+\sqrt{\hat{E}})(\|q\| \sqrt{\hat{E}}+\|\hat{q}\| \sqrt{E})}
\end{aligned}
$$

Therefore

$$
\begin{aligned}
& \frac{\left(\hat{t}_{h}-t_{h}\right)^{2} \gamma_{h} \bar{\gamma}_{h}+\left(t_{h}\|q\|-\hat{t}_{h}\|\hat{q}\|\right)^{2}}{\left(\hat{t}_{c}-t_{c}\right)^{2} \gamma_{c} \bar{\gamma}_{c}+\left(t_{c}\|q\|-\hat{t}_{c}\|\hat{q}\|\right)^{2}}=\frac{\left(\hat{t}_{h}-t_{h}\right)^{2} \gamma_{h} \bar{\gamma}_{h}+\left(t_{h}\|q\|-\hat{t}_{h}\|\hat{q}\|\right)^{2}}{\left(\hat{t}_{c}-t_{c}\right)^{2} \gamma_{c} \bar{\gamma}_{c}+\frac{\left|\gamma_{c}\right|^{2}}{m^{2}}\left(\hat{t}_{c}-t_{c}\right)^{2}} \\
& =\frac{\left(\hat{t}_{h}-t_{h}\right)^{2} \gamma_{h} \bar{\gamma}_{h}+\left(t_{h}\|q\|-\hat{t}_{h}\|\hat{q}\|\right)^{2}}{\left(\hat{t}_{c}-t_{c}\right)^{2}\left(\gamma_{c} \bar{\gamma}_{c}+\frac{\left|\gamma_{c}\right|^{2}}{m^{2}}\right)}=\frac{\left(\hat{t}_{h}-t_{h}\right)^{2} \gamma_{h} \bar{\gamma}_{h}+\left(t_{h}\|q\|-\hat{t}_{h}\|\hat{q}\|\right)^{2}}{\left(\frac{\alpha_{c}(\|q\|-\|\hat{q}\|)}{\left(\|\hat{q}\|+\frac{|| c \mid}{m}\right)\left(\|q\|+\frac{\left|\gamma_{c}\right|}{m}\right)}\right)^{2}\left(\gamma_{c} \bar{\gamma}_{c}+\frac{\left|\gamma_{c}\right|^{2}}{m^{2}}\right)} \\
& =\frac{\left(\frac{\alpha_{h}^{2}\left(\|q\|^{2}-\|\hat{q}\|^{2}\right)}{(\mathcal{C}+\sqrt{\hat{E}})(\mathcal{C}+\sqrt{E})(\sqrt{E}+\sqrt{\hat{E}})}\right)^{2} \gamma_{h} \bar{\gamma}_{h}}{\left(\frac{\alpha_{c}(\|q\|-\|\hat{q}\|)}{\left(\|\hat{q}\|+\frac{\left|\gamma_{c}\right|}{m}\right)\left(\|q\|+\frac{\left|\gamma_{c}\right|}{m}\right)}\right)^{2}\left(\gamma_{c} \bar{\gamma} c+\frac{\left|\gamma_{c}\right|^{2}}{m^{2}}\right)} \\
& +\frac{-\alpha_{h}\left(\mathcal{C}(\|q\|-\|\hat{q}\|)(\|q\| \sqrt{\hat{E}}+\|\hat{q}\| \sqrt{E})+\left(\mathcal{C}^{2}+\mathcal{C} \alpha_{h}\right)\left(\|q\|^{2}-\|\hat{q}\|^{2}\right)\right)}{(\mathcal{C}+\sqrt{E})(\mathcal{C}+\sqrt{\hat{E}})(\|q\| \sqrt{\hat{E}}+\|\hat{q}\| \sqrt{E})} \\
& \left.+\frac{\alpha_{c}(\|q\|-\|\hat{q}\|)}{\left(\|\hat{q}\|+\frac{\left|\gamma_{c}\right|}{m}\right)\left(\|q\|+\frac{\left|\gamma_{c}\right|}{m}\right)}\right)^{2}\left(\gamma_{c} \bar{\gamma}_{c}+\frac{\left|\gamma_{c}\right|^{2}}{m^{2}}\right)
\end{aligned}
$$




$$
\begin{gathered}
\left(\frac{\alpha_{h}^{2}(\|q\|+\|\hat{q}\|)}{(\mathcal{C}+\sqrt{\hat{E}})(\mathcal{C}+\sqrt{E})(\sqrt{E}+\sqrt{\hat{E}})}\right)^{2} \gamma_{h} \bar{\gamma}_{h} \\
\left(\frac{\alpha_{c}}{\left(\|\hat{q}\|+\frac{\left|\gamma_{c}\right|}{m}\right)\left(\|q\|+\frac{\left|\gamma_{c}\right|}{m}\right)}\right)^{2}\left(\gamma_{c} \bar{\gamma}_{c}+\frac{\left|\gamma_{c}\right|^{2}}{m^{2}}\right) \\
+\frac{\left(\frac{-\alpha_{h}\left(\mathcal{C}(\|q\| \sqrt{\hat{E}}+\|\hat{q}\| \sqrt{E})+\left(\mathcal{C}^{2}+\mathcal{C} \alpha_{h}\right)(\|q\|+\|\hat{q}\|)\right)}{(\mathcal{C}+\sqrt{E})(\mathcal{C}+\sqrt{\hat{E}})(\|q\| \sqrt{\hat{E}}+\|\hat{q}\| \sqrt{E})}\right)^{2}}{\left(\frac{\alpha_{c}}{\left(\|\hat{q}\|+\frac{\left|\gamma_{c}\right|}{m}\right)\left(\|q\|+\frac{\left|\gamma_{c}\right|}{m}\right)}\right)^{2}\left(\gamma_{c} \bar{\gamma}_{c}+\frac{\left|\gamma_{c}\right|^{2}}{m^{2}}\right)} \\
=\frac{\left(\|\hat{q}\|+\frac{\left|\gamma_{c}\right|}{m}\right)^{2}\left(\|q\|+\frac{\left|\gamma_{c}\right|}{m}\right)^{2}\left(\alpha_{h}^{2}(\|q\|+\|\hat{q}\|)\right)^{2} \gamma_{h} \bar{\gamma}_{h}}{\left(\alpha_{c}(\mathcal{C}+\sqrt{\hat{E}})(\mathcal{C}+\sqrt{E})(\sqrt{E}+\sqrt{\hat{E}})\right)^{2}\left(\gamma_{c} \bar{\gamma}_{c}+\frac{\left|\gamma_{c}\right|^{2}}{m^{2}}\right)} \\
+\frac{\left(\|\hat{q}\|+\frac{\left|\gamma_{c}\right|}{m}\right)^{2}\left(\|q\|+\frac{\left|\gamma_{c}\right|}{m}\right)^{2}}{\left(\alpha_{c}(\mathcal{C}+\sqrt{E})(\mathcal{C}+\sqrt{\hat{E}})\right)^{2}\left(\gamma_{c} \bar{\gamma}_{c}+\frac{\left|\gamma_{c}\right|^{2}}{m^{2}}\right)}\{ \\
\left(-\alpha_{h}\left(\mathcal{C}(\|q\| \sqrt{\hat{E}}+\|\hat{q}\| \sqrt{E})+\left(\mathcal{C}^{2}+\mathcal{C} \alpha_{h}\right)(\|q\|+\|\hat{q}\|)\right)\right)^{2} \\
(\|q\| \sqrt{\hat{E}}+\|\hat{q}\| \sqrt{E})^{2}
\end{gathered}
$$

We now apply lemmas 5.2.3, 5.2.4 and 5.2.5. Since $E=\mathcal{C}^{2}-\left(\|q\|^{2}-\mathcal{C}\right) \alpha_{h}$ and $\hat{E}=\mathcal{C}^{2}-\left(\|\hat{q}\|^{2}-\mathcal{C}\right) \alpha_{h}$, note that $\delta_{1}=\frac{\left|\gamma_{c}\right|}{m}>0, \delta_{2}=\delta_{3}=\mathcal{C}, \delta_{4}=$ $-\alpha_{h}>0, \delta_{5}=\mathcal{C}^{2}+\mathcal{C} \alpha_{h}>0$ and $\delta_{6}=-\mathcal{C} \alpha_{h}>0$. Also we can note that $\delta_{2}^{2}+\delta_{3} \delta_{4}>0$ and $\sqrt{\delta_{2}^{2}+\delta_{3} \delta_{4}}+\delta_{2}>0$. Thus

$$
\frac{\left(\hat{t}_{h}-t_{h}\right)^{2} \gamma_{h} \bar{\gamma}_{h}+\left(t_{h}\|q\|-\hat{t}_{h}\|\hat{q}\|\right)^{2}}{\left(\hat{t}_{c}-t_{c}\right)^{2} \gamma_{c} \bar{\gamma}_{c}+\left(t_{c}\|q\|-\hat{t}_{c}\|\hat{q}\|\right)^{2}}
$$

is bounded above and below. The proof is complete.

The conclusion proof of theorem (5.5.1) is following. By proposition 5.5.2, lemma 5.5.3 and theorem 5.2.2, the result follows.

Corollary 5.5.4 Any two extended hyperboloid metrics are equivalent.

Proof Consider any two extended hyperboloid metrics, then by theorem 5.3.1 and lemma 5.4.1, the result follows.

Corollary 5.5.5 Any extended paraboloid metric is equivalent to any extended hyperboloid metric. 


\subsection{Equivalence Between An Extended Sphere Metric and An Extended Ellipsoid Met- ric when $\alpha_{s} \neq 0$ and $\alpha_{e} \neq 0$}

Our goal is to show the equivalence between an extended sphere metric and an extended ellipsoid metric when $\alpha_{s} \neq 0$ and $\alpha_{e} \neq 0$.

Theorem 5.6.1 Any extended sphere metric with $\alpha_{s} \neq 0$ is equivalent to any extended ellipsoid metric with $\alpha_{e} \neq 0$.

Proof We proceed to prove theorem (5.6.1) by the following chain of lemmas and of propositions.

Proposition 5.6.2 The quantity

$$
\frac{\sqrt{F}-\sqrt{\hat{F}}}{\sqrt{\mathcal{E}}-\sqrt{\hat{\mathcal{E}}}}
$$

is bounded above and below, where

$$
\begin{gathered}
F=\frac{\mathcal{A}^{2}}{4}+\gamma_{s} \bar{\gamma}_{s} \alpha_{s}-\|q\|^{2} \alpha_{s}, \quad \hat{F}=\frac{\mathcal{A}^{2}}{4}+\gamma_{s} \bar{\gamma}_{s} \alpha_{s}-\|\hat{q}\|^{2} \alpha_{s}, \\
\mathcal{E}=\mathcal{D}^{2}+\mathcal{D} \alpha_{e}-\alpha_{e}\|q\|^{2} \quad \text { and } \hat{\mathcal{E}}=\mathcal{D}^{2}+\mathcal{D} \alpha_{e}-\alpha_{e}\|\hat{q}\|^{2}
\end{gathered}
$$

Proof Multiply by the conjugate to obtain

$$
\begin{aligned}
& \left(\frac{\sqrt{F}-\sqrt{\hat{F}}}{\sqrt{\mathcal{E}}-\sqrt{\hat{\mathcal{E}}}}\right)\left(\frac{\sqrt{F}+\sqrt{\hat{F}}}{\sqrt{\mathcal{E}}+\sqrt{\hat{\mathcal{E}}}}\right)\left(\frac{\sqrt{\mathcal{E}}+\sqrt{\hat{\mathcal{E}}}}{\sqrt{F}+\sqrt{\hat{F}}}\right) \\
& =\left(\frac{F-\hat{F}}{\mathcal{E}-\hat{\mathcal{E}}}\right)\left(\frac{\sqrt{\mathcal{E}}+\sqrt{\hat{\mathcal{E}}}}{\sqrt{F}+\sqrt{\hat{F}}}\right)
\end{aligned}
$$

For

$$
\frac{F-\hat{F}}{\mathcal{E}-\hat{\mathcal{E}}}
$$


we have

$$
\begin{aligned}
\frac{F-\hat{F}}{\mathcal{E}-\hat{\mathcal{E}}} & =\frac{\frac{\mathcal{A}^{2}}{4}-\left(\|q\|^{2}+\gamma_{s} \bar{\gamma}_{s}\right) \alpha_{s}-\frac{\mathcal{A}^{2}}{4}+\left(\|\hat{q}\|^{2}+\gamma_{s} \bar{\gamma}_{s}\right) \alpha_{s}}{\mathcal{D}^{2}-\left(\|q\|^{2}-\mathcal{D}\right) \alpha_{e}-\mathcal{D}^{2}+\left(\|\hat{q}\|^{2}-\mathcal{D}\right) \alpha_{e}} \\
& =\frac{-\alpha_{s}\left(\|q\|^{2}-\|\hat{q}\|^{2}\right)}{-\alpha_{e}\left(\|q\|^{2}-\|\hat{q}\|^{2}\right)}=\frac{\alpha_{s}}{\alpha_{e}}
\end{aligned}
$$

which is bounded above and below. Moreover, the denominator of $\frac{\mathcal{E}}{F}$ does not equal zero for all $\|q\|$, Compute the limit of $\frac{\mathcal{E}}{F}$ as $\|q\| \longrightarrow \infty$

$$
\frac{\mathcal{E}}{F}=\frac{\mathcal{D}^{2}-\left(\|q\|^{2}-\mathcal{D}\right) \alpha_{e}}{\frac{\mathcal{A}^{2}}{4}-\left(\|q\|^{2}+\gamma_{s} \bar{\gamma}_{s}\right) \alpha_{s}}
$$

which is a Möbius transformation thus $\frac{\mathcal{E}}{F}$ is bounded above and below. Therefore,

$$
0 \neq \mathcal{N}_{14} \leq \frac{\sqrt{\mathcal{E}}}{\sqrt{F}} \leq \mathcal{M}_{14} \neq \infty
$$

Similarly we can get

$$
0 \neq \mathcal{N}_{15} \leq \frac{\sqrt{\hat{\mathcal{E}}}}{\sqrt{\hat{F}}} \leq \mathcal{M}_{15} \neq \infty
$$

Thus

$$
\begin{aligned}
\frac{\sqrt{\mathcal{E}}+\sqrt{\hat{\mathcal{E}}}}{\sqrt{F}+\sqrt{\hat{F}}} & \leq \frac{\sqrt{F} \mathcal{M}_{14}+\sqrt{\hat{F}} \mathcal{M}_{15}}{\sqrt{F}+\sqrt{\hat{F}}} \leq \frac{\operatorname{Max}\left\{\mathcal{M}_{14}, \mathcal{M}_{15}\right\}(\sqrt{F}+\sqrt{\hat{F}})}{\sqrt{F}+\sqrt{\hat{F}}} \\
& =\operatorname{Max}\left\{\mathcal{M}_{14}, \mathcal{M}_{15}\right\}
\end{aligned}
$$

and

$$
\begin{aligned}
\frac{\sqrt{\mathcal{E}}+\sqrt{\hat{\mathcal{E}}}}{\sqrt{F}+\sqrt{\hat{F}}} \geq \frac{\sqrt{F} \mathcal{N}_{14}+\sqrt{\hat{F}} \mathcal{N}_{15}}{\sqrt{F}+\sqrt{\hat{F}}} \geq \frac{\min \left\{\mathcal{N}_{14}, \mathcal{N}_{15}\right\}\left(\sqrt{F_{1}}+\sqrt{\hat{F}_{1}}\right)}{\sqrt{F_{1}}+\sqrt{\hat{F}_{1}}} \\
=\min \left\{\mathcal{N}_{14}, \mathcal{N}_{15}\right\}
\end{aligned}
$$

Thus that the quantity

$$
\frac{\sqrt{F}-\sqrt{\hat{F}}}{\sqrt{\mathcal{E}}-\sqrt{\hat{\mathcal{E}}}}
$$


is bounded above and below, Namely

$$
0 \neq \mathcal{N}_{16} \leq \frac{\sqrt{F}-\sqrt{\hat{F}}}{\sqrt{\mathcal{E}}-\sqrt{\hat{\mathcal{E}}}} \leq \mathcal{M}_{16} \neq \infty
$$

The result follows.

Proposition 5.6.3 The quantities

$$
\frac{\sqrt{\hat{\mathcal{E}}}+\mathcal{D}}{\sqrt{\hat{F}}+\frac{\mathcal{A}}{2}} \text { and } \frac{\sqrt{\mathcal{E}}+\mathcal{D}}{\sqrt{F}+\frac{\mathcal{A}}{2}}
$$

are bounded above and below.

Proof For this purpose we multiply the quantity

$$
\frac{\sqrt{\hat{\mathcal{E}}}+\mathcal{D}}{\sqrt{\hat{F}}+\frac{\mathcal{A}}{2}}
$$

by the conjugate to obtain

$$
\begin{aligned}
\left(\frac{\hat{\mathcal{E}}-\mathcal{D}^{2}}{\hat{F}-\frac{\mathcal{A}^{2}}{4}}\right)\left(\frac{\sqrt{\hat{F}}-\frac{\mathcal{A}}{2}}{\sqrt{\hat{\mathcal{E}}}-\mathcal{D}}\right) & =\left(\frac{\mathcal{D}^{2}-\left(\|\hat{q}\|^{2}-\mathcal{D}\right) \alpha_{e}-\mathcal{D}^{2}}{\frac{\mathcal{A}^{2}}{4}-\left(\|\hat{q}\|^{2}+\gamma_{s} \bar{\gamma}_{s}\right) \alpha_{s}-\frac{\mathcal{A}^{2}}{4}}\right)\left(\frac{\sqrt{\hat{F}}-\frac{\mathcal{A}}{2}}{\sqrt{\hat{\mathcal{E}}}-\mathcal{D}}\right) \\
& =\left(\frac{-\left(\|\hat{q}\|^{2}-\mathcal{D}\right) \alpha_{e}}{-\left(\|\hat{q}\|^{2}+\gamma_{s} \bar{\gamma}_{s}\right) \alpha_{s}}\right)\left(\frac{\sqrt{\hat{F}}-\frac{\mathcal{A}}{2}}{\sqrt{\hat{\mathcal{E}}}-\mathcal{D}}\right)
\end{aligned}
$$

The quantity

$$
\frac{-\left(\|\hat{q}\|^{2}-\mathcal{D}\right) \alpha_{e}}{-\left(\|\hat{q}\|^{2}+\gamma_{s} \bar{\gamma}_{s}\right) \alpha_{s}}
$$

is a Möbius transformation, consequently, it is bounded above and below. From (5.6.2) we know that

$$
\frac{\sqrt{\hat{F}}}{\sqrt{\hat{\mathcal{E}}}}
$$

is bounded above and below. Also, $\frac{\mathcal{A}}{2 \mathcal{D}}$ is bounded above and below. Hence the result follows. 
Lemma 5.6.4 Assume

$$
t_{s}=\frac{-\alpha_{s}}{\sqrt{F}+\frac{\mathcal{A}}{2}}, \quad \hat{t}_{s}=\frac{-\alpha_{s}}{\sqrt{\hat{F}}+\frac{\mathcal{A}}{2}}
$$

and

$$
t_{e}=\frac{-\alpha_{e}}{\sqrt{\mathcal{E}}+\mathcal{D}}, \quad \hat{t}_{e}=\frac{-\alpha_{e}}{\sqrt{\hat{\mathcal{E}}}+\mathcal{D}}
$$

then the quantity

$$
\frac{\hat{t}_{s}-t_{s}}{\hat{t}_{e}-t_{e}}
$$

is bounded above and below.

Proof Compute

$$
\begin{aligned}
\frac{\hat{t}_{s}-t_{s}}{\hat{t}_{e}-t_{e}}=\frac{\frac{-\alpha_{s}(\sqrt{F}-\sqrt{\hat{F}})}{\left(\sqrt{\hat{F}}+\frac{\mathcal{A}}{2}\right)\left(\sqrt{F}+\frac{\mathcal{A}}{2}\right)}}{\frac{-\alpha_{e}(\sqrt{\mathcal{E}}-\sqrt{\hat{\mathcal{E}}})}{(\sqrt{\hat{\mathcal{E}}}+\mathcal{D})(\sqrt{\mathcal{E}}+\mathcal{D})}} \\
=\frac{-\alpha_{s}(\sqrt{F}-\sqrt{\hat{F}})(\sqrt{\hat{\mathcal{E}}}+\mathcal{D})(\sqrt{\mathcal{E}}+\mathcal{D})}{-\alpha_{e}(\sqrt{\mathcal{E}}-\sqrt{\hat{\mathcal{E}}})\left(\sqrt{\hat{F}}+\frac{\mathcal{A}}{2}\right)\left(\sqrt{F}+\frac{\mathcal{A}}{2}\right)}
\end{aligned}
$$

By the propositions 5.6.2 and 5.6.3, the result follows.

Lemma 5.6.5 the quantity

$$
\frac{\hat{t}_{s}\|\hat{q}\|-t_{s}\|q\|}{\hat{t}_{e}\|\hat{q}\|-t_{e}\|q\|}
$$

is bounded above and below.

Proof We determine various expressions for $\hat{t}_{s}\|\hat{q}\|-t_{s}\|q\|$ and $\hat{t}_{e}\|\hat{q}\|-t_{e}\|q\|$ that will be shown to be useful in the sequel.

$$
\begin{aligned}
\hat{t}_{s}\|\hat{q}\|-t_{s}\|q\| & =\frac{-\alpha_{s}\|\hat{q}\|}{\sqrt{\hat{F}}+\frac{\mathcal{A}}{2}}-\frac{-\alpha_{s}\|q\|}{\sqrt{F}+\frac{\mathcal{A}}{2}} \\
& =\frac{-\alpha_{s}\|\hat{q}\|\left(\sqrt{F}+\frac{\mathcal{A}}{2}\right)+\alpha_{s}\|q\|\left(\sqrt{\hat{F}}+\frac{\mathcal{A}}{2}\right)}{\left(\sqrt{\hat{F}}+\frac{\mathcal{A}}{2}\right)\left(\sqrt{F}+\frac{\mathcal{A}}{2}\right)}
\end{aligned}
$$


and

$$
\begin{aligned}
\hat{t}_{e}\|\hat{q}\|-t_{e}\|q\| & =\frac{-\alpha_{e}\|\hat{q}\|}{\sqrt{\hat{\mathcal{E}}}+\mathcal{D}}-\frac{-\alpha_{e}\|q\|}{\sqrt{\mathcal{E}}+\mathcal{D}} \\
& =\frac{-\alpha_{e}\|\hat{q}\|(\sqrt{\mathcal{E}}+\mathcal{D})+\alpha_{e}\|q\|(\sqrt{\hat{\mathcal{E}}}+\mathcal{D})}{(\sqrt{\hat{\mathcal{E}}}+\mathcal{D})(\sqrt{\mathcal{E}}+\mathcal{D})}
\end{aligned}
$$

Thus

$$
\begin{aligned}
& \frac{\hat{t}_{s}\|\hat{q}\|-t_{s}\|q\|}{\hat{t}_{e}\|\hat{q}\|-t_{e}\|q\|} \\
& =\frac{\left(-\alpha_{s}\|q\|\left(\sqrt{\hat{F}}+\frac{\mathcal{A}}{2}\right)+\alpha_{s}\|\hat{q}\|\left(\sqrt{F}+\frac{\mathcal{A}}{2}\right)\right)(\sqrt{\hat{\mathcal{E}}}+\mathcal{D})(\sqrt{\mathcal{E}}+\mathcal{D})}{\left(-\alpha_{e}\|q\|(\sqrt{\hat{\mathcal{E}}}+\mathcal{D})+\alpha_{e}\|\hat{q}\|(\sqrt{\mathcal{E}}+\mathcal{D})\right)\left(\sqrt{\hat{F}}+\frac{\mathcal{A}}{2}\right)\left(\sqrt{F}+\frac{\mathcal{A}}{2}\right)}
\end{aligned}
$$

By the propositions 5.6.2 and 5.6.3 we have the quantity

$$
\frac{(\sqrt{\hat{\mathcal{E}}}+\mathcal{D})(\sqrt{\mathcal{E}}+\mathcal{D})}{\left(\sqrt{\hat{F}}+\frac{\mathcal{A}}{2}\right)\left(\sqrt{F}+\frac{\mathcal{A}}{2}\right)}
$$

is bounded above and below. Next we will show that the quantity

$$
\frac{-\alpha_{s}\|q\|\left(\sqrt{\hat{F}}+\frac{\mathcal{A}}{2}\right)+\alpha_{s}\|\hat{q}\|\left(\sqrt{F}+\frac{\mathcal{A}}{2}\right)}{-\alpha_{e}\|q\|(\sqrt{\hat{\mathcal{E}}}+\mathcal{D})+\alpha_{e}\|\hat{q}\|(\sqrt{\mathcal{E}}+\mathcal{D})}
$$

is bounded above and below. Simplify the numerator of the preceding expression by using a simple trick to simplifying algebraic expressions, collecting like terms and multiplication by the conjugate, we get

$$
\begin{aligned}
& -\alpha_{s}\|q\|\left(\sqrt{\hat{F}}+\frac{\mathcal{A}}{2}\right)+\alpha_{s}\|\hat{q}\|\left(\sqrt{F}+\frac{\mathcal{A}}{2}\right) \\
& =\frac{-\alpha_{s}\left(\|q\|^{2}-\|\hat{q}\|^{2}\right)\left(\frac{\mathcal{A}^{2}}{4}-\alpha_{s} \gamma_{s} \bar{\gamma}_{s}\right)}{\|q\| \sqrt{\hat{F}}+\|\hat{q}\| \sqrt{F}}-\alpha_{s} \frac{\mathcal{A}}{2}(\|q\|-\|\hat{q}\|)
\end{aligned}
$$


Next simplify the denominator

$$
\begin{aligned}
& -\alpha_{e}\|q\|(\sqrt{\hat{\mathcal{E}}}+\mathcal{D})+\alpha_{e}\|\hat{q}\|(\sqrt{\mathcal{E}}+\mathcal{D}) \\
& =\frac{-\alpha_{e}\left(\|q\|^{2}-\|\hat{q}\|^{2}\right)\left(\mathcal{D}^{2}+\mathcal{D} \alpha_{e}\right)}{\|q\| \sqrt{\hat{\mathcal{E}}}+\|\hat{q}\| \sqrt{\mathcal{E}}}-\alpha_{e} \mathcal{D}(\|q\|-\|\hat{q}\|)
\end{aligned}
$$

Now the quantity

$$
\frac{-\alpha_{s} \frac{\mathcal{A}}{2}(\|q\|-\|\hat{q}\|)}{-\alpha_{e} \mathcal{D}(\|q\|-\|\hat{q}\|)}=\frac{-\alpha_{s_{1}} \frac{\mathcal{A}_{1}}{2}}{-\alpha_{e} \mathcal{D}}
$$

is bounded above and below. Moreover by proposition 5.6.2 we have the quantity

$$
\frac{\|q\| \sqrt{\hat{\mathcal{E}}}+\|\hat{q}\| \sqrt{\mathcal{E}}}{\|q\| \sqrt{\hat{F}}+\|\hat{q}\| \sqrt{F}}
$$

is bounded above and below, then

$$
\begin{aligned}
& \frac{-\alpha_{s}\left(\|q\|^{2}-\|\hat{q}\|^{2}\right)\left(\frac{\mathcal{A}^{2}}{4}-\alpha_{s} \gamma_{s} \bar{\gamma}_{s}\right)(\|q\| \sqrt{\hat{\mathcal{E}}}+\|\hat{q}\| \sqrt{\mathcal{E}})}{-\alpha_{e}\left(\|q\|^{2}-\|\hat{q}\|^{2}\right)\left(\mathcal{D}^{2}+\mathcal{D} \alpha_{e}\right)(\|q\| \sqrt{\hat{F}}+\|\hat{q}\| \sqrt{F})} \\
& =\frac{-\alpha_{s}\left(\frac{\mathcal{A}^{2}}{4}-\alpha_{s} \gamma_{s} \bar{\gamma}_{s}\right)(\|q\| \sqrt{\hat{\mathcal{E}}}+\|\hat{q}\| \sqrt{\mathcal{E}})}{-\alpha_{e}\left(\mathcal{D}^{2}+\mathcal{D} \alpha_{e}\right)(\|q\| \sqrt{\hat{F}}+\|\hat{q}\| \sqrt{F})}
\end{aligned}
$$

is bounded above and below. Hence the proof is complete.

The conclusion of the proof of theorem (5.6.1) follows. By lemmas 5.6.4, 5.6.5 and theorem 5.2.1 we can show that the extended sphere metric is equivalent to the extended ellipsoid metric when $\alpha_{s} \neq 0$ and $\alpha_{e} \neq 0$ and the result follows.

\subsection{Equivalence Between An Extended Sphere Metric with $\alpha_{s}=0$ and An Extended El- lipsoid Metric with $\alpha_{e}=0$}

We will show the equivalent between the extended sphere metric with $\alpha_{s}=0$ and the extended ellipsoid metric whit $\alpha_{e}=0$. 
Theorem 5.7.1 Any extended sphere metric with $\alpha_{s}=0$ is equivalent to any extended ellipsoid metric with $\alpha_{e}=0$

Proof We proceed to prove theorem (5.7.1) by the following chain lemmas.

Lemma 5.7.2 Assume

$$
t_{s_{0}}=\frac{-\mathcal{A}_{0}}{\|q\|^{2}+\gamma_{s_{0}} \bar{\gamma}_{s_{0}}} \quad t_{e_{0}}=\frac{-2 \mathcal{D}_{0}}{\|q\|^{2}-\mathcal{D}_{0}}
$$

with $\alpha_{e}=0$ and $\alpha_{s}=0$ then the quantity $\frac{t_{s_{0}}}{t_{e_{0}}}$ bounded above and below.

Proof Compute the limit of $\frac{t_{s_{0}}}{t_{e_{0}}}$ as $\|q\| \longrightarrow \infty$

$$
\begin{aligned}
\lim _{\|q\| \longrightarrow \infty} \frac{t_{s_{0}}}{t_{e_{0}}}= & \lim _{\|q\| \longrightarrow \infty} \frac{\frac{-\mathcal{A}_{0}}{\|q\|^{2}+\gamma_{s_{0}} \bar{\gamma}_{s_{0}}}}{\frac{-2 \mathcal{D}_{0}}{\|q\|^{2}-\mathcal{D}_{0}}}=\lim _{\|q\| \longrightarrow \infty} \frac{-\mathcal{A}_{0}\left(\|q\|^{2}-\mathcal{D}_{0}\right)}{-2 \mathcal{D}_{0}\left(\|q\|^{2}+\gamma_{s_{0}} \bar{\gamma}_{s_{0}}\right)} \\
& =\lim _{\|q\| \longrightarrow \infty} \frac{\mathcal{A}_{0}\left(1-\frac{\mathcal{D}_{0}}{\|q\|^{2}}\right)}{2 \mathcal{D}_{0}\left(1+\frac{\gamma_{s_{0}} \bar{\gamma}_{s_{0}}}{\|q\|^{2}}\right)}=\frac{\mathcal{A}_{0}}{2 \mathcal{D}_{0}}>0
\end{aligned}
$$

By definition of limit we have for all $\epsilon>0$, then there exist $\rho>0$ such that for all $\|q\|>\rho$ we have

$$
\frac{\mathcal{A}_{0}}{2 \mathcal{D}_{0}}-\epsilon \leq \frac{-\mathcal{A}_{0}\left(\|q\|^{2}-\mathcal{D}_{0}\right)}{-2 \mathcal{D}_{0}\left(\|q\|^{2}+\gamma_{s_{0}} \bar{\gamma}_{s_{0}}\right)} \leq \frac{\mathcal{A}_{0}}{2 \mathcal{D}_{0}}+\epsilon
$$

Compute the limit of $\frac{t_{s_{0}}}{t_{e_{0}}}$ as $\|q\| \longrightarrow 0$

$$
\lim _{\|q\| \longrightarrow 0} \frac{\mathcal{A}_{0}\left(\|q\|^{2}-\mathcal{D}_{0}\right)}{2 \mathcal{D}_{0}\left(\|q\|^{2}+\gamma_{s_{0}} \bar{\gamma}_{s_{0}}\right)}=\frac{-\mathcal{A}_{0} \mathcal{D}_{0}}{2 \mathcal{D}_{0} \gamma_{s_{0}} \bar{\gamma}_{s_{0}}}=\frac{-\mathcal{A}_{0}}{2 \gamma_{s_{0}} \bar{\gamma}_{s_{0}}}>0
$$

Given any $\epsilon>0$, then there exist $\rho>0$ such that for all $\|q\|<\rho$ we have

$$
\frac{-\mathcal{A}_{0}}{2 \gamma_{s_{0}} \bar{\gamma}_{s_{0}}}-\epsilon \leq \frac{-\mathcal{A}_{0}\left(\|q\|^{2}-\mathcal{D}_{0}\right)}{-2 \mathcal{D}_{0}\left(\|q\|^{2}+\gamma_{s_{0}} \bar{\gamma}_{s_{0}}\right)} \leq \frac{-\mathcal{A}_{0}}{2 \gamma_{s_{0}} \bar{\gamma}_{s_{0}}}+\epsilon
$$

Moreover, the denominator of $\frac{t_{s_{0}}}{t_{e_{0}}}$ does not equal zero for all $\|q\|$, also, the numerator and the denominator are positive and bounded on $[\hat{\rho}, \tilde{\rho}]$. Hence the quotient is a continuous function of $\|q\|$ on the closed interval $[\hat{\rho}, \tilde{\rho}]$, this implies that the quotient has a positive minimum and maximum on $[\hat{\rho}, \tilde{\rho}]$. The proof is complete. 
Lemma 5.7.3 The ratio

$$
\frac{\left(\hat{t}_{s_{0}}-t_{s_{0}}\right)^{2} \gamma_{s_{0}} \bar{\gamma}_{s_{0}}+\left(t_{s_{0}}\|q\|-\hat{t}_{s_{0}}\|\hat{q}\|\right)^{2}}{\left(\hat{t}_{e_{0}}-t_{e_{0}}\right)^{2} \gamma_{e_{0}} \bar{\gamma}_{e_{0}}+\left(t_{e_{0}}\|q\|-\hat{t}_{e_{0}}\|\hat{q}\|\right)^{2}}
$$

is bounded above and below.

Proof We first determine various expressions for $\hat{t}_{s_{0}}-t_{s_{0}}, \hat{t}_{s_{0}}\|\hat{q}\|-t_{s_{0}}\|q\|$, $\hat{t}_{e_{0}}-t_{e_{0}}$ and $\hat{t}_{e_{0}}\|\hat{q}\|-t_{e_{0}}\|q\|$ that will be shown to be useful in the sequel. When $\alpha_{e}=0$ we have

$$
t_{e_{0}}=\frac{-2 \mathcal{D}_{0}}{\|q\|^{2}-\mathcal{D}_{0}} \quad \hat{t}_{e_{0}}=\frac{-2 \mathcal{D}_{0}}{\|\hat{q}\|^{2}-\mathcal{D}_{0}}
$$

therefore

$$
\begin{aligned}
& \hat{t}_{e_{0}}-t_{e_{0}}=\frac{-2 \mathcal{D}_{0}}{\|\hat{q}\|^{2}-\mathcal{D}_{0}}-\frac{-2 \mathcal{D}_{0}}{\|q\|^{2}-\mathcal{D}_{0}} \\
& =\frac{\left[\|q\|^{2}-\mathcal{D}_{0}\right]\left[-2 \mathcal{D}_{0}\right]-\left[\|\hat{q}\|^{2}-\mathcal{D}_{0}\right]\left[-2 \mathcal{D}_{0}\right]}{\left[\|q\|^{2}-\mathcal{D}_{0}\right]\left[\|\hat{q}\|^{2}-\mathcal{D}_{0}\right]} \\
& =\frac{-2 \mathcal{D}_{0}\left(\|q\|^{2}-\|\hat{q}\|^{2}\right)}{\left[\|q\|^{2}-\mathcal{D}_{0}\right]\left[\|\hat{q}\|^{2}-\mathcal{D}_{0}\right]}
\end{aligned}
$$

and

$$
\begin{aligned}
& \hat{t}_{e_{0}}\|\hat{q}\|-t_{e_{0}}\|q\|=\frac{-2 \mathcal{D}_{0}\|\hat{q}\|}{\|\hat{q}\|^{2}-\mathcal{D}_{0}}-\frac{-2 \mathcal{D}_{0}\|q\|}{\|q\|^{2}-\mathcal{D}_{0}} \\
& =\frac{\left[\|q\|^{2}-\mathcal{D}_{0}\right]\left[-2 \mathcal{D}_{0}\right]\|\hat{q}\|-\left[\|\hat{q}\|^{2}-\mathcal{D}_{0}\right]\left[-2 \mathcal{D}_{0}\right]\|q\|}{\left[\|q\|^{2}-\mathcal{D}_{0}\right]\left[\|\hat{q}\|^{2}-\mathcal{D}_{0}\right]} \\
& =\frac{-2 \mathcal{D}_{0}\|\hat{q}\|\|q\|^{2}+2 \mathcal{D}_{0}^{2}\|\hat{q}\|+2 \mathcal{D}_{0}\|q\|\|\hat{q}\|^{2}-2 \mathcal{D}_{0}^{2}\|q\|}{\left[\|q\|^{2}-\mathcal{D}_{0}\right]\left[\|\hat{q}\|^{2}-\mathcal{D}_{0}\right]} \\
& =\frac{-2 \mathcal{D}_{0}\|\hat{q}\|\|q\|(\|q\|-\|\hat{q}\|)-2 \mathcal{D}_{0}^{2}(\|q\|-\|\hat{q}\|)}{\left[\|q\|^{2}-\mathcal{D}_{0}\right]\left[\|\hat{q}\|^{2}-\mathcal{D}_{0}\right]} \\
& =\frac{-2 \mathcal{D}_{0}(\|q\|-\|\hat{q}\|)\left(\|\hat{q}\|\|q\|+\mathcal{D}_{0}\right)}{\left[\|q\|^{2}-\mathcal{D}_{0}\right]\left[\|\hat{q}\|^{2}-\mathcal{D}_{0}\right]}
\end{aligned}
$$

Also when $\alpha_{s}=0$ we have

$$
t_{s_{0}}=\frac{-\mathcal{A}_{0}}{\|q\|^{2}+\gamma_{s_{0}} \bar{\gamma}_{s_{0}}} \quad \hat{t}_{s_{0}}=\frac{-\mathcal{A}_{0}}{\|\hat{q}\|^{2}+\gamma_{s_{0}} \bar{\gamma}_{s_{0}}}
$$


therefore

$$
\begin{aligned}
& \hat{t}_{s_{0}}-t_{s_{0}}=\frac{-\mathcal{A}_{0}}{\|\hat{q}\|^{2}+\gamma_{s_{0}} \bar{\gamma}_{s_{0}}}-\frac{-\mathcal{A}_{0}}{\|q\|^{2}+\gamma_{s_{0}} \bar{\gamma}_{s_{0}}} \\
& =\frac{\left[\|q\|^{2}+\gamma_{s_{0}} \bar{\gamma}_{s_{0}}\right]\left[-\mathcal{A}_{0}\right]-\left[\|\hat{q}\|^{2}+\gamma_{s_{0}} \bar{\gamma}_{s_{0}}\right]\left[-\mathcal{A}_{0}\right]}{\left[\|q\|^{2}+\gamma_{s_{0}} \bar{\gamma}_{s_{0}}\right]\left[\|\hat{q}\|^{2}+\gamma_{s_{0}} \bar{\gamma}_{s_{0}}\right]} \\
& =\frac{-\mathcal{A}_{0}\left(\|q\|^{2}-\|\hat{q}\|^{2}\right)}{\left[\|q\|^{2}+\gamma_{s_{0}} \bar{\gamma}_{s_{0}}\right]\left[\|\hat{q}\|^{2}+\gamma_{s_{0}} \bar{\gamma}_{s_{0}}\right]} .
\end{aligned}
$$

and

$$
\begin{aligned}
& \hat{t}_{s_{0}}\|\hat{q}\|-t_{s_{0}}\|q\|=\frac{-\mathcal{A}_{0}\|\hat{q}\|}{\|\hat{q}\|^{2}+\gamma_{s_{0}} \bar{\gamma}_{s_{0}}}-\frac{-\mathcal{A}_{0}\|q\|}{\|q\|^{2}+\gamma_{s_{0}} \bar{\gamma}_{s_{0}}} \\
& =\frac{\left[\|q\|^{2}+\gamma_{s_{0}} \bar{\gamma}_{s_{0}}\right]\left[-\mathcal{A}_{0}\right]\|\hat{q}\|-\left[\|\hat{q}\|^{2}+\gamma_{s_{0}} \bar{\gamma}_{s_{0}}\right]\left[-\mathcal{A}_{0}\right]\|q\|}{\left[\|q\|^{2}+\gamma_{s_{0}} \bar{\gamma}_{s_{0}}\right]\left[\|\hat{q}\|^{2}+\gamma_{s_{0}} \bar{\gamma}_{s_{0}}\right]} \\
& =\frac{-\mathcal{A}_{0}\|\hat{q}\|\|q\|^{2}-\mathcal{A}_{0}\|\hat{q}\| \gamma_{s_{0}} \bar{\gamma}_{s_{0}}+\mathcal{A}_{0}\|q\|\|\hat{q}\|^{2}+\mathcal{A}_{0}\|q\| \gamma_{s_{0}} \bar{\gamma}_{s_{0}}}{\left[\|q\|^{2}+\gamma_{s_{0}} \bar{\gamma}_{s_{0}}\right]\left[\|\hat{q}\|^{2}+\gamma_{s_{0}} \bar{\gamma}_{s_{0}}\right]} \\
& =\frac{-\mathcal{A}_{0}\|\hat{q}\|\|q\|(\|q\|-\|\hat{q}\|)+\mathcal{A}_{0} \gamma_{s_{0}} \bar{\gamma}_{s_{0}}(\|q\|-\|\hat{q}\|)}{\left[\|q\|^{2}+\gamma_{s_{0}} \bar{\gamma}_{s_{0}}\right]\left[\|\hat{q}\|^{2}+\gamma_{s_{0}} \bar{\gamma}_{s_{0}}\right]} \\
& =\frac{-\mathcal{A}_{0}(\|q\|-\|\hat{q}\|)\left(\|\hat{q}\|\|q\|-\gamma_{s_{0}} \bar{\gamma}_{s_{0}}\right)}{\left[\|q\|^{2}+\gamma_{s_{0}} \bar{\gamma}_{s_{0}}\right]\left[\|\hat{q}\|^{2}+\gamma_{s_{0}} \bar{\gamma}_{s_{0}}\right]} .
\end{aligned}
$$


Thus

$$
\begin{aligned}
& \frac{\left(\hat{t}_{s_{0}}-t_{s_{0}}\right)^{2} \gamma_{s_{0}} \bar{\gamma}_{s_{0}}+\left(t_{s_{0}}\|q\|-\hat{t}_{s_{0}}\|\hat{q}\|\right)^{2}}{\left(\hat{t}_{e_{0}}-t_{e_{0}}\right)^{2} \gamma_{e_{0}} \bar{\gamma}_{e_{0}}+\left(t_{e_{0}}\|q\|-\hat{t}_{e_{0}}\|\hat{q}\|\right)^{2}} \\
& =\frac{\left(\frac{-\mathcal{A}_{0}\left(\|q\|^{2}-\|\hat{q}\|^{2}\right)}{\left[\|q\|^{2}+\gamma_{s_{0}} \bar{\gamma}_{s_{0}}\right]\left[\|\hat{q}\|^{2}+\gamma_{s_{0}} \bar{\gamma}_{s_{0}}\right]}\right)^{2} \gamma_{s_{0}} \bar{\gamma}_{s_{0}}+\left(\frac{-\mathcal{A}_{0}(\|q\|-\|\hat{q}\|)\left(\|\hat{q}\|\|q\|-\gamma_{s_{0}} \bar{\gamma}_{s_{0}}\right)}{\left[\|q\|^{2}+\gamma_{s_{0}} \bar{\gamma}_{s_{0}}\right]\left[\|\hat{q}\|^{2}+\gamma_{s_{0}} \bar{\gamma}_{s_{0}}\right]}\right)^{2}}{\left(\frac{-2 \mathcal{D}_{0}\left(\|q\|^{2}-\|\hat{q}\|^{2}\right)}{\left[\|q\|^{2}-\mathcal{D}_{0}\right]\left[\|\hat{q}\|^{2}-\mathcal{D}_{0}\right]}\right)^{2} \gamma_{e_{0}} \bar{\gamma}_{e_{0}}+\left(\frac{-2 \mathcal{D}_{0}(\|q\|-\|\hat{q}\|)\left(\|\hat{q}\|\|q\|+\mathcal{D}_{0}\right)}{\left[\|q\|^{2}-\mathcal{D}_{0}\right]\left[\|\hat{q}\|^{2}-\mathcal{D}_{0}\right]}\right)^{2}} \\
& =\frac{\left(\frac{-\mathcal{A}_{0}(\|q\|+\|\hat{q}\|)}{\left[\|q\|^{2}+\gamma_{s_{0}} \bar{\gamma}_{s_{0}}\right]\left[\|\hat{q}\|^{2}+\gamma_{s_{0}} \bar{\gamma}_{s_{0}}\right]}\right)^{2} \gamma_{s_{0}} \bar{\gamma}_{s_{0}}+\left(\frac{-\mathcal{A}_{0}\left(\|\hat{q}\|\|q\|-\gamma_{s_{0}} \bar{\gamma}_{s_{0}}\right)}{\left[\|q\|^{2}+\gamma_{s_{0}} \bar{\gamma}_{s_{0}}\right]\left[\|\hat{q}\|^{2}+\gamma_{s_{0}} \bar{\gamma}_{s_{0}}\right]}\right)^{2}}{\left(\frac{-2 \mathcal{D}_{0}(\|q\|+\|\hat{q}\|)}{\left[\|q\|^{2}-\mathcal{D}_{0}\right]\left[\|\hat{q}\|^{2}-\mathcal{D}_{0}\right]}\right)^{2} \gamma_{e_{0}} \bar{\gamma}_{e_{0}}+\left(\frac{-2 \mathcal{D}_{0}\left(\|\hat{q}\|\|q\|+\mathcal{D}_{0}\right)}{\left[\|q\|^{2}-\mathcal{D}_{0}\right]\left[\|\hat{q}\|^{2}-\mathcal{D}_{0}\right]}\right)^{2}} \\
& =\left(\frac{\left[\|q\|^{2}-\mathcal{D}_{0}\right]\left[\|\hat{q}\|^{2}-\mathcal{D}_{0}\right]}{\left[\|q\|^{2}+\gamma_{s_{0}} \bar{\gamma}_{s_{0}}\right]\left[\|\hat{q}\|^{2}+\gamma_{s_{0}} \bar{\gamma}_{s_{0}}\right]}\right)^{2}\{ \\
& \left.\frac{\left(-\mathcal{A}_{0}(\|q\|+\|\hat{q}\|)\right)^{2} \gamma_{s_{0}} \bar{\gamma}_{s_{0}}+\left(-\mathcal{A}_{0}\left(\|\hat{q}\|\|q\|-\gamma_{s_{0}} \bar{\gamma}_{s_{0}}\right)\right)^{2}}{\left(-2 \mathcal{D}_{0}(\|q\|+\|\hat{q}\|)\right)^{2} \gamma_{e_{0}} \bar{\gamma}_{e_{0}}+\left(-2 \mathcal{D}_{0}\left(\|\hat{q}\|\|q\|+\mathcal{D}_{0}\right)\right)^{2}}\right\}
\end{aligned}
$$

Next we apply the limit when $\|q\| \longrightarrow \infty$ and $\|\hat{q}\| \longrightarrow \infty$,

$$
\begin{aligned}
& \lim _{\substack{\|q\| \rightarrow \infty \\
\|\hat{q}\| \longrightarrow \infty}}\left(\frac{\left[\|q\|^{2}-\mathcal{D}_{0}\right]\left[\|\hat{q}\|^{2}-\mathcal{D}_{0}\right]}{\left[\|q\|^{2}+\gamma_{s_{0}} \bar{\gamma}_{s_{0}}\right]\left[\|\hat{q}\|^{2}+\gamma_{s_{0}} \bar{\gamma}_{s_{0}}\right]}\right)^{2}\{ \\
& \left.\frac{\left(-\mathcal{A}_{0}(\|q\|+\|\hat{q}\|)\right)^{2} \gamma_{s_{0}} \bar{\gamma}_{s_{0}}+\left(-\mathcal{A}_{0}\left(\|\hat{q}\|\|q\|-\gamma_{s_{0}} \bar{\gamma}_{s_{0}}\right)\right)^{2}}{\left(-2 \mathcal{D}_{0}(\|q\|+\|\hat{q}\|)\right)^{2} \gamma_{e_{0}} \bar{\gamma}_{e_{0}}+\left(-2 \mathcal{D}_{0}\left(\|\hat{q}\|\|q\|+\mathcal{D}_{0}\right)\right)^{2}}\right\} \\
& =\frac{\mathcal{A}_{0}^{2}}{4 \mathcal{D}_{0}^{2}}>0
\end{aligned}
$$

Also we apply the limit when $\|q\| \longrightarrow 0$ and $\|\hat{q}\| \longrightarrow 0$,

$$
\begin{aligned}
& \lim _{\substack{\|q\| \rightarrow 0 \\
\|\hat{q}\| \longrightarrow 0}}\left(\frac{\left[\|q\|^{2}-\mathcal{D}_{0}\right]\left[\|\hat{q}\|^{2}-\mathcal{D}_{0}\right]}{\left[\|q\|^{2}+\gamma_{s_{0}} \bar{\gamma}_{s_{0}}\right]\left[\|\hat{q}\|^{2}+\gamma_{s_{0}} \bar{\gamma}_{s_{0}}\right]}\right)^{2}\{ \\
& \left.\frac{\left(-\mathcal{A}_{0}(\|q\|+\|\hat{q}\|)\right)^{2} \gamma_{s_{0}} \bar{\gamma}_{s_{0}}+\left(-\mathcal{A}_{0}\left(\|\hat{q}\|\|q\|-\gamma_{s_{0}} \bar{\gamma}_{s_{0}}\right)\right)^{2}}{\left(-2 \mathcal{D}_{0}(\|q\|+\|\hat{q}\|)\right)^{2} \gamma_{e_{0}} \bar{\gamma}_{e_{0}}+\left(-2 \mathcal{D}_{0}\left(\|\hat{q}\|\|q\|+\mathcal{D}_{0}\right)\right)^{2}}\right\} \\
& =\frac{\mathcal{D}_{0}^{4}}{\left[\gamma_{s_{0}} \bar{\gamma}_{s_{0}}\right]^{2}}\left\{\frac{\left(\mathcal{A}_{0} \gamma_{s_{0}} \bar{\gamma}_{s_{0}}\right)^{2}}{\left(-2 \mathcal{D}_{0}\right)^{4}}\right\}=\frac{\mathcal{D}_{0}^{2} \mathcal{A}_{0}^{2}}{4 \gamma_{s_{0}} \bar{\gamma}_{s_{0}}}>0
\end{aligned}
$$


Moreover, the denominator of

$$
\frac{\left(\hat{t}_{s_{0}}-t_{s_{0}}\right)^{2} \gamma_{s_{0}} \bar{\gamma}_{s_{0}}+\left(t_{s_{0}}\|q\|-\hat{t}_{s_{0}}\|\hat{q}\|\right)^{2}}{\left(\hat{t}_{e_{0}}-t_{e_{0}}\right)^{2} \gamma_{e_{0}} \bar{\gamma}_{e_{0}}+\left(t_{e_{0}}\|q\|-\hat{t}_{e_{0}}\|\hat{q}\|\right)^{2}}
$$

does not equal zero for all $\|q\|$ and $\|\hat{q}\|$, thus it is a continuous function on a closed, bounded set in $\mathbb{R}^{2}$, then it attains an absolute maximum value at some points in this set. The proof is complete.

The conclusion proof of theorem (5.7.1) as following. By lemmas 5.7.2, 5.7.3 and theorem 5.2.2, the result follows.

\subsection{Equivalence Between Two Extended Sphere Metrics when $\alpha_{s} \neq 0$}

For any sphere we know that and

$$
t_{s}=\frac{-\frac{\mathcal{A}}{2}+\sqrt{F}}{\|q\|^{2}+\gamma_{s} \bar{\gamma}_{s}} .
$$

Put for a first sphere

$$
\begin{gathered}
\mathcal{A}_{1}=-2 \gamma_{s_{1}} \bar{\gamma}_{s_{1}}+\gamma_{s_{1}} \bar{\theta}_{s_{1}}+\theta_{s_{1}} \bar{\gamma}_{s_{1}} \\
\alpha_{s_{1}}=\gamma_{s_{1}} \bar{\gamma}_{s_{1}}-\gamma_{s_{1}} \bar{\theta}_{s_{1}}-\theta_{s_{1}} \bar{\gamma}_{s_{1}}+\theta_{s_{1}} \bar{\theta}_{s_{1}}-R^{2}=\left(\gamma_{s_{1}}-\theta_{s_{1}}\right) \overline{\left(\gamma_{s_{1}}-\theta_{s_{1}}\right)}-R^{2} \\
F_{1}=\frac{\mathcal{A}_{1}^{2}}{4}-\left(\|q\|^{2}+\gamma_{s_{1}} \bar{\gamma}_{s_{1}}\right) \alpha_{s_{1}}
\end{gathered}
$$

and

$$
t_{s_{1}}=\frac{-\frac{\mathcal{A}_{1}}{2}+\sqrt{F_{1}}}{\|q\|^{2}+\gamma_{s_{1}} \bar{\gamma}_{s_{1}}} .
$$

also for a second sphere

$$
\begin{gathered}
\mathcal{A}_{2}=-2 \gamma_{s_{2}} \bar{\gamma}_{s_{2}}+\gamma_{s_{2}} \bar{\theta}_{s_{2}}+\theta_{s_{2}} \bar{\gamma}_{s_{2}} \\
\alpha_{s_{2}}=\gamma_{s_{2}} \bar{\gamma}_{s_{2}}-\gamma_{s_{2}} \bar{\theta}_{s_{2}}-\theta_{s_{2}} \bar{\gamma}_{s_{2}}+\theta_{s_{2}} \bar{\theta}_{s_{2}}-R^{2}=\left(\gamma_{s_{2}}-\theta_{s_{2}}\right) \overline{\left(\gamma_{s_{2}}-\theta_{s_{2}}\right)}-R^{2} \\
F_{2}=\frac{\mathcal{A}_{2}^{2}}{4}-\left(\|q\|^{2}+\gamma_{s_{2}} \bar{\gamma}_{s_{2}}\right) \alpha_{s_{2}}
\end{gathered}
$$


and

$$
t_{s_{2}}=\frac{-\frac{\mathcal{A}_{2}}{2}+\sqrt{F_{2}}}{\|q\|^{2}+\gamma_{s_{2}} \bar{\gamma}_{s_{2}}} .
$$

When $\alpha_{s_{1}} \neq 0$ and $\alpha_{s_{2}} \neq 0$ then we have

$$
t_{s_{1}}=\frac{-\alpha_{s_{1}}}{\sqrt{F_{1}}+\frac{\mathcal{A}_{1}}{2}} .
$$

also

$$
t_{s_{2}}=\frac{-\alpha_{s_{2}}}{\sqrt{F_{2}}+\frac{\mathcal{A}_{2}}{2}} .
$$

We first determine various expressions for $\hat{t}_{s_{1}}-t_{s_{1}}$ and $\hat{t}_{s_{1}}\|\hat{q}\|-t_{s_{1}}\|q\|$ that will be shown to be useful in the sequel.

$$
\begin{aligned}
\hat{t}_{s_{1}}-t_{s_{1}}= & \frac{-\alpha_{s_{1}}}{\sqrt{\hat{F}_{1}}+\frac{\mathcal{A}_{1}}{2}}-\frac{-\alpha_{s_{1}}}{\sqrt{F_{1}}+\frac{\mathcal{A}_{1}}{2}}=\frac{-\alpha_{s_{1}}\left(\sqrt{F_{1}}+\frac{\mathcal{A}_{1}}{2}\right)+\alpha_{s_{1}}\left(\sqrt{\hat{F}_{1}}+\frac{\mathcal{A}_{1}}{2}\right)}{\left(\sqrt{\hat{F}_{1}}+\frac{\mathcal{A}_{1}}{2}\right)\left(\sqrt{F_{1}}+\frac{\mathcal{A}_{1}}{2}\right)} \\
= & \frac{-\alpha_{s_{1}} \sqrt{F_{1}}+\alpha_{s_{1}} \sqrt{\hat{F}_{1}}}{\left(\sqrt{\hat{F}_{1}}+\frac{\mathcal{A}_{1}}{2}\right)\left(\sqrt{F_{1}}+\frac{\mathcal{A}_{1}}{2}\right)}=\frac{-\alpha_{s_{1}}\left(\sqrt{F_{1}}-\sqrt{\hat{F}_{1}}\right)}{\left(\sqrt{\hat{F}_{1}}+\frac{\mathcal{A}_{1}}{2}\right)\left(\sqrt{F_{1}}+\frac{\mathcal{A}_{1}}{2}\right)}
\end{aligned}
$$

and

$$
\begin{aligned}
t_{s_{1}}\|q\|-\hat{t}_{s_{1}}\|\hat{q}\| & =\frac{-\alpha_{s_{1}}\|q\|}{\sqrt{F_{1}}+\frac{\mathcal{A}_{1}}{2}}-\frac{-\alpha_{s_{1}}\|\hat{q}\|}{\sqrt{\hat{F}_{1}}+\frac{\mathcal{A}_{1}}{2}} \\
& =\frac{-\alpha_{s_{1}}\|q\|\left(\sqrt{\hat{F}_{1}}+\frac{\mathcal{A}_{1}}{2}\right)+\alpha_{s_{1}}\|\hat{q}\|\left(\sqrt{F_{1}}+\frac{\mathcal{A}_{1}}{2}\right)}{\left(\sqrt{\hat{F}_{1}}+\frac{\mathcal{A}_{1}}{2}\right)\left(\sqrt{F_{1}}+\frac{\mathcal{A}_{1}}{2}\right)}
\end{aligned}
$$

Theorem 5.8.1 Any two distinct extended sphere metrics when $\alpha_{s} \neq 0$ are equivalent.

Proof We proceed to prove theorem (5.8.1) by the following chain lemmas.

Lemma 5.8.2 The quantity

$$
\frac{\sqrt{F_{1}}-\sqrt{\hat{F}_{1}}}{\sqrt{F_{2}}-\sqrt{\hat{F}_{2}}}
$$

is bounded above and below 
Proof For this purpose we multiply the quantity

$$
\frac{\sqrt{F_{1}}-\sqrt{\hat{F}_{1}}}{\sqrt{F_{2}}-\sqrt{\hat{F}_{2}}}
$$

by the conjugate to obtain

$$
\begin{aligned}
& \left(\frac{\sqrt{F_{1}}-\sqrt{\hat{F}_{1}}}{\sqrt{F_{2}}-\sqrt{\hat{F}_{2}}}\right)\left(\frac{\sqrt{F_{1}}+\sqrt{\hat{F}_{1}}}{\sqrt{F_{2}}+\sqrt{\hat{F}_{2}}}\right)\left(\frac{\sqrt{F_{2}}+\sqrt{\hat{F}_{2}}}{\sqrt{F_{1}}+\sqrt{\hat{F}_{1}}}\right) \\
& =\left(\frac{F_{1}-\hat{F}_{1}}{F_{2}-\hat{F}_{2}}\right)\left(\frac{\sqrt{F_{2}}+\sqrt{\hat{F}_{2}}}{\sqrt{F_{1}}+\sqrt{\hat{F}_{1}}}\right)
\end{aligned}
$$

For

$$
\frac{F_{1}-\hat{F}_{1}}{F_{2}-\hat{F}_{2}}
$$

we have

$$
\begin{aligned}
\frac{F_{1}-\hat{F}_{1}}{F_{2}-\hat{F}_{2}} & =\frac{\frac{\mathcal{A}_{1}^{2}}{4}-\left(\|q\|^{2}+\gamma_{s_{1}} \bar{\gamma}_{s_{1}}\right) \alpha_{s_{1}}-\frac{\mathcal{A}_{1}^{2}}{4}+\left(\|\hat{q}\|^{2}+\gamma_{s_{1}} \bar{\gamma}_{s_{1}}\right) \alpha_{s_{1}}}{\frac{\mathcal{A}_{2}^{2}}{4}-\left(\|q\|^{2}+\gamma_{s_{2}} \bar{\gamma}_{s_{2}}\right) \alpha_{s_{2}}-\frac{\mathcal{A}_{2}^{2}}{4}+\left(\|\hat{q}\|^{2}+\gamma_{s_{2}} \bar{\gamma}_{s_{2}}\right) \alpha_{s_{2}}} \\
& =\frac{-\alpha_{s_{1}}\left(\|q\|^{2}-\|\hat{q}\|^{2}\right)}{-\alpha_{s_{2}}\left(\|q\|^{2}-\|\hat{q}\|^{2}\right)}=\frac{\alpha_{s_{1}}}{\alpha_{s_{2}}}
\end{aligned}
$$

which is bounded above and below. Now

$$
\frac{F_{2}}{F_{1}}=\frac{\frac{\mathcal{A}_{2}^{2}}{4}-\left(\|q\|^{2}+\gamma_{s_{2}} \bar{\gamma}_{s_{2}}\right) \alpha_{s_{2}}}{\frac{\mathcal{A}_{1}^{2}}{4}-\left(\|q\|^{2}+\gamma_{s_{1}} \bar{\gamma}_{s_{1}}\right) \alpha_{s_{1}}}=\frac{\frac{\mathcal{A}_{2}^{2}}{4}-\alpha_{s_{2}} \gamma_{s_{2}} \bar{\gamma}_{s_{2}}-\alpha_{s_{2}}\|q\|^{2}}{\frac{\mathcal{A}_{1}^{2}}{4}-\alpha_{s_{1}} \gamma_{s_{1}} \bar{\gamma}_{s_{1}}-\alpha_{s_{1}}\|q\|^{2}}
$$

which is a Möbius transformation thus $\frac{F_{2}}{F_{1}}$ is bounded above and below. Therefore,

$$
0 \neq \mathcal{N}_{14} \leq \frac{\sqrt{F_{2}}}{\sqrt{F_{1}}} \leq \mathcal{M}_{14} \neq \infty
$$

Similar we can get

$$
0 \neq \mathcal{N}_{15} \leq \frac{\sqrt{\hat{F}_{2}}}{\sqrt{\hat{F}_{1}}} \leq \mathcal{M}_{15} \neq \infty
$$

Thus 


$$
\begin{aligned}
\frac{\sqrt{F_{2}}+\sqrt{\hat{F}_{2}}}{\sqrt{F_{1}}+\sqrt{\hat{F}_{1}}} & \leq \frac{\sqrt{F_{1}} \mathcal{M}_{8}+\sqrt{\hat{F}_{1}} \mathcal{M}_{9}}{\sqrt{F_{1}}+\sqrt{\hat{F}_{1}}} \leq \frac{\operatorname{Max}\left\{\mathcal{M}_{8}, \mathcal{M}_{9}\right\}\left(\sqrt{F_{1}}+\sqrt{\hat{F}_{1}}\right)}{\sqrt{F_{1}}+\sqrt{\hat{F}_{1}}} \\
& =\operatorname{Max}\left\{\mathcal{M}_{14}, \mathcal{M}_{15}\right\}
\end{aligned}
$$

and

$$
\begin{aligned}
\frac{\sqrt{F_{2}}+\sqrt{\hat{F}_{2}}}{\sqrt{F_{1}}+\sqrt{\hat{F}_{1}}} & \geq \frac{\sqrt{F_{1}} \mathcal{N}_{8}+\sqrt{\hat{F}_{1}} \mathcal{N}_{9}}{\sqrt{F_{1}}+\sqrt{\hat{F}_{1}}} \geq \frac{\min \left\{\mathcal{N}_{8}, \mathcal{N}_{9}\right\}\left(\sqrt{F_{1}}+\sqrt{\hat{F}_{1}}\right)}{\sqrt{F_{1}}+\sqrt{\hat{F}_{1}}} \\
& =\min \left\{\mathcal{N}_{14}, \mathcal{N}_{15}\right\}
\end{aligned}
$$

Thus that the quantity

$$
\frac{\sqrt{F_{1}}-\sqrt{\hat{F}_{1}}}{\sqrt{F_{2}}-\sqrt{\hat{F}_{2}}}
$$

is bounded above and below. The proof complete.

Lemma 5.8.3 The quantities

$$
\frac{\sqrt{\hat{F}_{2}}+\frac{\mathcal{A}_{2}}{2}}{\sqrt{\hat{F}_{1}}+\frac{\mathcal{A}_{1}}{2}} \text { and } \frac{\sqrt{F_{2}}+\frac{\mathcal{A}_{2}}{2}}{\sqrt{F_{1}}+\frac{\mathcal{A}_{1}}{2}}
$$

are bounded above and below.

Proof For this purpose we multiply the quantity

$$
\frac{\sqrt{\hat{F}_{2}}+\frac{\mathcal{A}_{2}}{2}}{\sqrt{\hat{F}_{1}}+\frac{\mathcal{A}_{1}}{2}}
$$

by the conjugate to obtain

$$
\begin{aligned}
\left(\frac{\hat{F}_{2}+\frac{\mathcal{A}_{2}^{2}}{4}}{\hat{F}_{1}+\frac{\mathcal{A}_{1}^{2}}{4}}\right)\left(\frac{\sqrt{\hat{F}_{1}}-\frac{\mathcal{A}_{1}}{2}}{\sqrt{\hat{F}_{2}}-\frac{\mathcal{A}_{2}}{2}}\right) & =\left(\frac{\frac{\mathcal{A}_{2}^{2}}{4}-\left(\|\hat{q}\|^{2}+\gamma_{s_{2}} \bar{\gamma}_{s_{2}}\right) \alpha_{s_{2}}-\frac{\mathcal{A}_{2}^{2}}{4}}{\frac{\mathcal{A}_{1}^{2}}{4}-\left(\|\hat{q}\|^{2}+\gamma_{s_{1}} \bar{\gamma}_{s_{1}}\right) \alpha_{s_{1}}-\frac{\mathcal{A}_{1}^{2}}{4}}\right)\left(\frac{\sqrt{\hat{F}_{1}}-\frac{\mathcal{A}_{1}}{2}}{\sqrt{\hat{F}_{2}}-\frac{\mathcal{A}_{2}}{2}}\right) \\
& =\left(\frac{-\left(\|\hat{q}\|^{2}+\gamma_{s_{2}} \bar{\gamma}_{s_{2}}\right) \alpha_{s_{2}}}{-\left(\|\hat{q}\|^{2}+\gamma_{s_{1}} \bar{\gamma}_{s_{1}}\right) \alpha_{s_{1}}}\right)\left(\frac{\sqrt{\hat{F}_{1}}-\frac{\mathcal{A}_{1}}{2}}{\sqrt{\hat{F}_{2}}-\frac{\mathcal{A}_{2}}{2}}\right)
\end{aligned}
$$


The quantity

$$
\frac{\left(\|\hat{q}\|^{2}+\gamma_{s_{2}} \bar{\gamma}_{s_{2}}\right) \alpha_{s_{2}}}{\left(\|\hat{q}\|^{2}+\gamma_{s_{1}} \bar{\gamma}_{s_{1}}\right) \alpha_{s_{1}}}
$$

is a Möbius transformation, consequently, it is bounded above and below. From (5.8.1) we know that

$$
\frac{\sqrt{\hat{F}_{1}}}{\sqrt{\hat{F}_{2}}}
$$

is bounded above and below. Also, $\frac{\mathcal{A}_{1}}{\mathcal{A}_{2}}$ is bounded above and below. Hence the result follows.

Lemma 5.8.4 Assume

$$
t_{s_{1}}=\frac{-\alpha_{s_{1}}}{\sqrt{F_{1}}+\frac{\mathcal{A}_{1}}{2}} \quad, \hat{t}_{s_{1}}=\frac{-\alpha_{s_{1}}}{\sqrt{\hat{F}_{1}}+\frac{\mathcal{A}_{1}}{2}}
$$

and

$$
t_{s 2}=\frac{-\alpha_{s_{2}}}{\sqrt{F_{2}}+\frac{\mathcal{A}_{2}}{2}} \quad, \hat{t}_{s_{2}}=\frac{-\alpha_{s_{2}}}{\sqrt{\hat{F}_{2}+\frac{\mathcal{A}_{2}}{2}}}
$$

then the quantity

$$
\left|\frac{\hat{t}_{s_{1}}-t_{s_{1}}}{\hat{t}_{s_{2}}-t_{s_{2}}}\right|
$$

is bounded above and below.

\section{Proof}

$$
\begin{aligned}
\frac{\hat{t}_{s_{1}}-t_{s_{1}}}{\hat{t}_{s_{2}}-t_{s_{2}}}= & \frac{\frac{-\alpha_{s_{1}}\left(\sqrt{F_{1}}-\sqrt{\hat{F}_{1}}\right)}{\left(\sqrt{\hat{F}_{1}}+\frac{\mathcal{A}_{1}}{2}\right)\left(\sqrt{F_{1}}+\frac{\mathcal{A}_{1}}{2}\right)}}{\frac{-\alpha_{s_{2}}\left(\sqrt{F_{2}}-\sqrt{\hat{F}_{2}}\right)}{\left(\sqrt{\hat{F}_{2}}+\frac{\mathcal{A}_{2}}{2}\right)\left(\sqrt{F_{2}}+\frac{\mathcal{A}_{2}}{2}\right)}} \\
= & \frac{-\alpha_{s_{1}}\left(\sqrt{F_{1}}-\sqrt{\hat{F}_{1}}\right)\left(\sqrt{\hat{F}_{2}}+\frac{\mathcal{A}_{2}}{2}\right)\left(\sqrt{F_{2}}+\frac{\mathcal{A}_{2}}{2}\right)}{-\alpha_{s_{2}}\left(\sqrt{F_{2}}-\sqrt{\hat{F}_{2}}\right)\left(\sqrt{\hat{F}_{1}}+\frac{\mathcal{A}_{1}}{2}\right)\left(\sqrt{F_{1}}+\frac{\mathcal{A}_{1}}{2}\right)}
\end{aligned}
$$

By lemmas 5.8.2 and 5.8.3, the result follows. 
Lemma 5.8.5 Assume

$$
t_{s_{1}}=\frac{-\alpha_{s_{1}}}{\sqrt{F_{1}}+\frac{\mathcal{A}_{1}}{2}} \quad, \hat{t}_{s_{1}}=\frac{-\alpha_{s_{1}}}{\sqrt{\hat{F}_{1}}+\frac{\mathcal{A}_{1}}{2}}
$$

and

$$
t_{s_{2}}=\frac{-\alpha_{s_{2}}}{\sqrt{F_{2}}+\frac{\mathcal{A}_{2}}{2}} \quad, \hat{t}_{s_{2}}=\frac{-\alpha_{s_{2}}}{\sqrt{\hat{F}_{2}}+\frac{\mathcal{A}_{2}}{2}}
$$

then the quantity

$$
\frac{\hat{t}_{s_{1}}\|\hat{q}\|-t_{s_{1}}\|q\|}{\hat{t}_{s_{2}}\|\hat{q}\|-t_{s_{2}}\|q\|}
$$

is bounded, namely

$$
0<\mathcal{N}_{12} \leq \frac{\hat{t}_{s_{1}}\|\hat{q}\|-t_{s_{1}}\|q\|}{\hat{t}_{s_{2}}\|\hat{q}\|-t_{s_{2}}\|q\|} \leq \mathcal{M}_{12}<\infty
$$

\section{Proof}

$$
\begin{aligned}
& \frac{\hat{t}_{s_{1}}\|\hat{q}\|-t_{s_{1}}\|q\|}{\hat{t}_{s_{2}}\|\hat{q}\|-t_{s_{2}}\|q\|} \\
& =\frac{\left(-\alpha_{s_{1}}\|q\|\left(\sqrt{\hat{F}_{1}}+\frac{\mathcal{A}_{1}}{2}\right)+\alpha_{s_{1}}\|\hat{q}\|\left(\sqrt{F_{1}}+\frac{\mathcal{A}_{1}}{2}\right)\right)\left(\sqrt{\hat{F}_{2}}+\frac{\mathcal{A}_{2}}{2}\right)\left(\sqrt{F_{2}}+\frac{\mathcal{A}_{2}}{2}\right)}{\left(-\alpha_{s_{2}}\|q\|\left(\sqrt{\hat{F}_{2}}+\frac{\mathcal{A}_{2}}{2}\right)+\alpha_{s_{2}}\|\hat{q}\|\left(\sqrt{F_{2}}+\frac{\mathcal{A}_{2}}{2}\right)\right)\left(\sqrt{\hat{F}_{1}}+\frac{\mathcal{A}_{1}}{2}\right)\left(\sqrt{F_{1}}+\frac{\mathcal{A}_{1}}{2}\right)}
\end{aligned}
$$

From the preceding proposition we know that the quantity

$$
\frac{\left(\sqrt{\hat{F}_{2}}+\frac{\mathcal{A}_{2}}{2}\right)\left(\sqrt{F_{2}}+\frac{\mathcal{A}_{2}}{2}\right)}{\left(\sqrt{\hat{F}_{1}}+\frac{\mathcal{A}_{1}}{2}\right)\left(\sqrt{F_{1}}+\frac{\mathcal{A}_{1}}{2}\right)}
$$

is bounded. Next we will show that the quantity

$$
\frac{-\alpha_{s_{1}}\|q\|\left(\sqrt{\hat{F}_{1}}+\frac{\mathcal{A}_{1}}{2}\right)+\alpha_{s_{1}}\|\hat{q}\|\left(\sqrt{F_{1}}+\frac{\mathcal{A}_{1}}{2}\right)}{-\alpha_{s_{2}}\|q\|\left(\sqrt{\hat{F}_{2}}+\frac{\mathcal{A}_{2}}{2}\right)+\alpha_{s_{2}}\|\hat{q}\|\left(\sqrt{F_{2}}+\frac{\mathcal{A}_{2}}{2}\right)}
$$

is bounded. To reach our goal some simplification is needed, so to simplify the preceding expression we use a simple trick worth remembering we multiplying out brackets, simplifying algebraic expressions, collecting like terms 
and multiplication by the conjugate. First we simplify the numerator

$$
\begin{aligned}
& -\alpha_{s_{1}}\|q\|\left(\sqrt{\hat{F}_{1}}+\frac{\mathcal{A}_{1}}{2}\right)+\alpha_{s_{1}}\|\hat{q}\|\left(\sqrt{F_{1}}+\frac{\mathcal{A}_{1}}{2}\right) \\
& =-\alpha_{s_{1}}\|q\| \sqrt{\hat{F}_{1}}-\alpha_{s_{1}}\|q\| \frac{\mathcal{A}_{1}}{2}+\alpha_{s_{1}}\|\hat{q}\| \sqrt{F_{1}}+\alpha_{s_{1}}\|\hat{q}\| \frac{\mathcal{A}_{1}}{2} \\
& =-\alpha_{s_{1}}\left(\|q\| \sqrt{\hat{F}_{1}}-\|\hat{q}\| \sqrt{F_{1}}\right)-\alpha_{s_{1}} \frac{\mathcal{A}_{1}}{2}(\|q\|-\|\hat{q}\|) \\
& =\frac{-\alpha_{s_{1}}\left(\|q\| \sqrt{\hat{F}_{1}}-\|\hat{q}\| \sqrt{F_{1}}\right)\left(\|q\| \sqrt{\hat{F}_{1}}+\|\hat{q}\| \sqrt{F_{1}}\right)}{\|q\| \sqrt{\hat{F}_{1}}+\|\hat{q}\| \sqrt{F_{1}}}-\alpha_{s_{1}} \frac{\mathcal{A}_{1}}{2}(\|q\|-\|\hat{q}\|) \\
& =\frac{-\alpha_{s_{1}}\left(\|q\|^{2}\left(\frac{\mathcal{A}_{1}^{2}}{4}-\alpha_{s_{1}}\left(\|\hat{q}\|^{2}+\gamma_{s_{1}} \bar{\gamma}_{s_{1}}\right)\right)-\|\hat{q}\|^{2}\left(\frac{\mathcal{A}_{1}^{2}}{4}-\alpha_{s_{1}}\left(\|q\|^{2}+\gamma_{s_{1}} \bar{\gamma}_{s_{1}}\right)\right)\right)}{\|q\| \sqrt{\hat{F}_{1}}+\|\hat{q}\| \sqrt{F_{1}}} \\
& -\alpha_{s_{1}} \frac{\mathcal{A}_{1}}{2}(\|q\|-\|\hat{q}\|) \\
& =\frac{-\alpha_{s_{1}}\left(\|q\|^{2}\left(\frac{\mathcal{A}_{1}^{2}}{4}-\alpha_{s_{1}} \gamma_{s_{1}} \bar{\gamma}_{s_{1}}\right)-\|\hat{q}\|^{2}\left(\frac{\mathcal{A}_{1}^{2}}{4}-\alpha_{s_{1}} \gamma_{s_{1}} \bar{\gamma}_{s_{1}}\right)\right)}{\|q\| \sqrt{\hat{F}_{1}}+\|\hat{q}\| \sqrt{F_{1}}} \\
& -\alpha_{s_{1}} \frac{\mathcal{A}_{1}}{2}(\|q\|-\|\hat{q}\|) \\
& =\frac{-\alpha_{s_{1}}\left(\|q\|^{2}-\|\hat{q}\|^{2}\right)\left(\frac{\mathcal{A}_{1}^{2}}{4}-\alpha_{s_{1}} \gamma_{s_{1}} \bar{\gamma}_{s_{1}}\right)}{\|q\| \sqrt{\hat{F}_{1}}+\|\hat{q}\| \sqrt{F_{1}}}-\alpha_{s_{1}} \frac{\mathcal{A}_{1}}{2}(\|q\|-\|\hat{q}\|)
\end{aligned}
$$


Second we simplify the denominator

$$
\begin{aligned}
- & \alpha_{s_{2}}\|q\|\left(\sqrt{\hat{F}_{2}}+\frac{\mathcal{A}_{2}}{2}\right)+\alpha_{s_{2}}\|\hat{q}\|\left(\sqrt{F_{2}}+\frac{\mathcal{A}_{2}}{2}\right) \\
= & -\alpha_{s_{2}}\|q\| \sqrt{\hat{F}_{2}}-\alpha_{s_{2}}\|q\| \frac{\mathcal{A}_{2}}{2}+\alpha_{s_{2}}\|\hat{q}\| \sqrt{F_{2}}+\alpha_{s_{2}}\|\hat{q}\| \frac{\mathcal{A}_{2}}{2} \\
= & -\alpha_{s_{2}}\left(\|q\| \sqrt{\hat{F}_{2}}-\|\hat{q}\| \sqrt{F_{2}}\right)-\alpha_{s_{2}} \frac{\mathcal{A}_{2}}{2}(\|q\|-\|\hat{q}\|) \\
= & \frac{-\alpha_{s_{2}}\left(\|q\| \sqrt{\hat{F}_{2}}-\|\hat{q}\| \sqrt{F_{2}}\right)\left(\|q\| \sqrt{\hat{F}_{2}}+\|\hat{q}\| \sqrt{F_{2}}\right)}{\|q\| \sqrt{\hat{F}_{2}}+\|\hat{q}\| \sqrt{F_{2}}}-\alpha_{s_{2}} \frac{\mathcal{A}_{2}}{2}(\|q\|-\|\hat{q}\|) \\
= & \frac{-\alpha_{s_{2}}\left(\|q\|^{2}\left(\frac{\mathcal{A}_{2}^{2}}{4}-\alpha_{s_{2}}\left(\|\hat{q}\|^{2}+\gamma_{s_{2}} \bar{\gamma}_{s_{2}}\right)\right)-\|\hat{q}\|^{2}\left(\frac{\mathcal{A}_{2}^{2}}{4}-\alpha_{s_{2}}\left(\|q\|^{2}+\gamma_{s_{2}} \bar{\gamma}_{s_{2}}\right)\right)\right)}{\|q\| \sqrt{\hat{F}_{2}}+\|\hat{q}\| \sqrt{F_{2}}} \\
- & \alpha_{s_{2}} \frac{\mathcal{A}_{2}}{2}(\|q\|-\|\hat{q}\|) \\
= & \frac{-\alpha_{s_{2}}\left(\|q\|^{2}\left(\frac{\mathcal{A}_{2}^{2}}{4}-\alpha_{s_{2}} \gamma_{s_{2}} \bar{\gamma}_{s_{2}}\right)-\|\hat{q}\|^{2}\left(\frac{\mathcal{A}_{2}^{2}}{4}-\alpha_{s_{2}} \gamma_{s_{2}} \bar{\gamma}_{s_{2}}\right)\right)}{\|q\| \sqrt{\hat{F}_{2}}+\|\hat{q}\| \sqrt{F_{2}}} \\
- & \alpha_{s_{2}} \frac{\mathcal{A}_{2}}{2}(\|q\|-\|\hat{q}\|) \\
= & \frac{-\alpha_{s_{2}}\left(\|q\|^{2}-\|\hat{q}\|^{2}\right)\left(\frac{\mathcal{A}_{2}^{2}}{4}-\alpha_{s_{2}} \gamma_{s_{2}} \bar{\gamma}_{s_{2}}\right)}{\|q\| \sqrt{\hat{F_{2}}}+\|\hat{q}\| \sqrt{F_{2}}}-\alpha_{s_{2}} \frac{\mathcal{A}_{2}}{2}(\|q\|-\|\hat{q}\|) \\
= &
\end{aligned}
$$

Since

$$
\frac{-\alpha_{s_{1}} \frac{\mathcal{A}_{1}}{2}(\|q\|-\|\hat{q}\|)}{-\alpha_{s_{2}} \frac{\mathcal{A}_{2}}{2}(\|q\|-\|\hat{q}\|)}=\frac{-\alpha_{s_{1}} \frac{\mathcal{A}_{1}}{2}}{-\alpha_{s_{2}} \frac{\mathcal{A}_{2}}{2}}
$$

is bounded. Now we will show that

$$
\frac{\frac{-\alpha_{s_{1}}\left(\|q\|^{2}-\|\hat{q}\|^{2}\right)\left(\frac{\mathcal{A}_{1}^{2}}{4}-\alpha_{s_{1}} \gamma_{s_{1}} \bar{\gamma}_{s_{1}}\right)}{\|q\| \sqrt{\hat{F}_{1}}+\|\hat{q}\| \sqrt{F_{1}}}}{\frac{-\alpha_{s_{2}}\left(\|q\|^{2}-\|\hat{q}\|^{2}\right)\left(\frac{\mathcal{A}_{2}^{2}}{4}-\alpha_{s_{2}} \gamma_{s_{2}} \bar{\gamma}_{s_{2}}\right)}{\|q\| \sqrt{\hat{F}_{2}}+\|\hat{q}\| \sqrt{F_{2}}}}
$$


is bounded above and below,

$$
\begin{gathered}
\frac{-\alpha_{s_{1}}\left(\|q\|^{2}-\|\hat{q}\|^{2}\right)\left(\frac{\mathcal{A}_{1}^{2}}{4}-\alpha_{s_{1}} \gamma_{s_{1}} \bar{\gamma}_{s_{1}}\right)\left(\|q\| \sqrt{\hat{F}_{2}}+\|\hat{q}\| \sqrt{F_{2}}\right)}{-\alpha_{s_{2}}\left(\|q\|^{2}-\|\hat{q}\|^{2}\right)\left(\frac{\mathcal{A}_{2}^{2}}{4}-\alpha_{s_{2}} \gamma_{s_{2}} \bar{\gamma}_{s_{2}}\right)\left(\|q\| \sqrt{\hat{F}_{1}}+\|\hat{q}\| \sqrt{F_{1}}\right)} \\
=\frac{-\alpha_{s_{1}}(\|q\|+\|\hat{q}\|)\left(\frac{\mathcal{A}_{1}^{2}}{4}-\alpha_{s_{1}} \gamma_{s_{1}} \bar{\gamma}_{s_{1}}\right)\left(\|q\| \sqrt{\hat{F}_{2}}+\|\hat{q}\| \sqrt{F_{2}}\right)}{-\alpha_{s_{2}}(\|q\|+\|\hat{q}\|)\left(\frac{\mathcal{A}_{2}^{2}}{4}-\alpha_{s_{2}} \gamma_{s_{2}} \bar{\gamma}_{s_{2}}\right)\left(\|q\| \sqrt{\hat{F}_{1}}+\|\hat{q}\| \sqrt{F_{1}}\right)} \\
-\alpha_{s_{1}}\|q\|\left(\sqrt{\hat{F}_{1}}+\frac{\mathcal{A}_{1}}{2}\right)+\alpha_{s_{1}}\|\hat{q}\|\left(\sqrt{F_{1}}+\frac{\mathcal{A}_{1}}{2}\right) \\
-\alpha_{s_{2}}\|q\|\left(\sqrt{\hat{F}_{2}}+\frac{\mathcal{A}_{2}}{2}\right)+\alpha_{s_{2}}\|\hat{q}\|\left(\sqrt{F_{2}}+\frac{\mathcal{A}_{2}}{2}\right)
\end{gathered}
$$

The result follows.

The conclusion proof of theorem (5.8.1) as following. By lemmas 5.8.4, 5.8.5 and theorem 5.2.1 we can show that The spherically metrics when $\alpha_{s}=0$ and the ellipsoid metrics when $\alpha_{e}=0$ are equivalence, the result follows.

Corollary 5.8.6 Any two distinct extended ellipsoid metric when $\alpha_{e} \neq 0$ are equivalent.

\subsection{Equivalence Between Two Extended Sphere Metrics when $\alpha_{s}=0$}

We will show the equivalence two between sphere when $\alpha_{s}=0$ metric. We start with the following proposition.

Proposition 5.9.1 Assume $\alpha_{s}=0$ with
1. $t_{s_{0} 1}=\frac{-\mathcal{A}_{01}}{\|q\|^{2}+\gamma_{s_{01}} \bar{\gamma}_{s_{01}}}$
2. $t_{s_{0} 2}=\frac{-\mathcal{A}_{02}}{\|q\|^{2}+\gamma_{s_{02}} \bar{\gamma}_{s_{02}}}$

then the quantity $\frac{t_{s_{01}}}{t_{s_{02}}}$ is bounded above and below.

Proof Compute $\frac{t_{s_{01}}}{t_{s_{02}}}$

$$
\frac{t_{s_{01}}}{t_{s_{02}}}=\lim _{\|q\| \longrightarrow \infty} \frac{\frac{-\mathcal{A}_{01}}{\|q\|^{2}+\gamma_{s_{01}} \bar{\gamma}_{s_{01}}}}{\frac{-\mathcal{A}_{02}}{\|q\|^{2}+\gamma_{s_{02}} \bar{\gamma}_{s_{02}}}}=\frac{-\mathcal{A}_{01}\left(\|q\|^{2}+\gamma_{s_{02}} \bar{\gamma}_{s_{02}}\right)}{-\mathcal{A}_{02}\left(\|q\|^{2}+\gamma_{s_{01}} \bar{\gamma}_{s_{01}}\right)}
$$


which is a Möbius transformation, consequently, it is bounded above and below. The proof is complete.

Lemma 5.9.2 Assume
1. $t_{s_{01}}=\frac{-\mathcal{A}_{01}}{\|q\|^{2}+\gamma_{s_{01}} \bar{\gamma}_{s_{01}}}$
3. $t_{s_{02}}=\frac{-\mathcal{A}_{02}}{\|q\|^{2}+\gamma_{s_{02}} \bar{\gamma}_{s_{02}}}$
2. $\hat{t}_{s_{01}}=\frac{-\mathcal{A}_{01}}{\|\hat{q}\|^{2}+\gamma_{s_{01}} \bar{\gamma}_{s_{01}}}$
4. $\hat{t}_{s_{02}}=\frac{-\mathcal{A}_{02}}{\|\hat{q}\|^{2}+\gamma_{s_{02}} \bar{\gamma}_{s_{02}}}$
with $\alpha_{s}=0$, then the ratio

$$
\frac{\left(\hat{t}_{s_{01}}-t_{s_{01}}\right)^{2} \gamma_{s_{01}} \bar{\gamma}_{s_{01}}+\left(t_{s_{01}}\|q\|-\hat{t}_{s_{01}}\|\hat{q}\|\right)^{2}}{\left(\hat{t}_{s_{02}}-t_{s_{02}}\right)^{2} \gamma_{s_{02}} \bar{\gamma}_{s_{02}}+\left(t_{s_{02}}\|q\|-\hat{t}_{s_{02}}\|\hat{q}\|\right)^{2}}
$$

is bounded for all $\|q\|$ and $\|\hat{q}\|$. Namely for some real positive $\mathcal{K}_{1}, \mathcal{K}_{2}$ we have

$$
0<\mathcal{K}_{1} \leq \frac{\left(\hat{t}_{s_{01}}-t_{s_{01}}\right)^{2} \gamma_{s_{01}} \bar{\gamma}_{s_{01}}+\left(t_{s_{01}}\|q\|-\hat{t}_{s_{01}}\|\hat{q}\|\right)^{2}}{\left(\hat{t}_{s_{02}}-t_{s_{02}}\right)^{2} \gamma_{s_{02}} \bar{\gamma}_{s_{02}}+\left(t_{s_{02}}\|q\|-\hat{t}_{s_{02}}\|\hat{q}\|\right)^{2}} \leq \mathcal{K}_{2}
$$

Proof We first determine various expressions for $\hat{t}_{s_{01}}-t_{s_{01}}, t_{s_{01}}\|q\|-\hat{t}_{s_{01}}\|\hat{q}\|$, $\hat{t}_{s_{02}}-t_{s_{02}}$ and $t_{s_{02}}\|q\|-\hat{t}_{s_{02}}\|\hat{q}\|$ that will be shown to be useful in the sequel. therefore

$$
\begin{aligned}
& t_{s_{01}}-\hat{t}_{s_{01}}=\frac{-\mathcal{A}_{01}}{\|\hat{q}\|^{2}+\gamma_{s_{01}} \bar{\gamma}_{s_{01}}}-\frac{-\mathcal{A}_{01}}{\|q\|^{2}+\gamma_{s_{01}} \bar{\gamma}_{s_{01}}} \\
& =\frac{\left[\|q\|^{2}+\gamma_{s_{01}} \bar{\gamma}_{s_{01}}\right]\left[-\mathcal{A}_{01}\right]-\left[\|\hat{q}\|^{2}+\gamma_{s_{01}} \bar{\gamma}_{s_{01}}\right]\left[-\mathcal{A}_{01}\right]}{\left[\|q\|^{2}+\gamma_{s_{01}} \bar{\gamma}_{s_{01}}\right]\left[\|\hat{q}\|^{2}+\gamma_{s_{01}} \bar{\gamma}_{s_{01}}\right]} \\
& =\frac{-\mathcal{A}_{01}\left(\|q\|^{2}-\|\hat{q}\|^{2}\right)}{\left[\|q\|^{2}+\gamma_{s_{01}} \bar{\gamma}_{s_{01}}\right]\left[\|\hat{q}\|^{2}+\gamma_{s_{01}} \bar{\gamma}_{s_{01}}\right]}
\end{aligned}
$$


and

$$
\begin{aligned}
& t_{s_{01}}\|q\|-\hat{t}_{s_{01}}\|\hat{q}\|=\frac{-\mathcal{A}_{01}\|\hat{q}\|}{\|\hat{q}\|^{2}+\gamma_{s_{01}} \bar{\gamma}_{s_{01}}}-\frac{-\mathcal{A}_{01}\|q\|}{\|q\|^{2}+\gamma_{s_{01}} \bar{\gamma}_{s_{01}}} \\
& =\frac{\left[\|q\|^{2}+\gamma_{s_{01}} \bar{\gamma}_{s_{01}}\right]\left[-\mathcal{A}_{01}\right]\|\hat{q}\|-\left[\|\hat{q}\|^{2}+\gamma_{s_{01}} \bar{\gamma}_{s_{01}}\right]\left[-\mathcal{A}_{01}\right]\|q\|}{\left[\|q\|^{2}+\gamma_{s_{01}} \bar{\gamma}_{s_{01}}\right]\left[\|\hat{q}\|^{2}+\gamma_{s_{01}} \bar{\gamma}_{s_{01}}\right]} \\
& =\frac{-\mathcal{A}_{01}\|\hat{q}\|\|q\|^{2}-\mathcal{A}_{01}\|\hat{q}\| \gamma_{s_{01}} \bar{\gamma}_{s_{01}}+\mathcal{A}_{01}\|q\|\|\hat{q}\|^{2}+\mathcal{A}_{01}\|q\| \gamma_{s_{01}} \bar{\gamma}_{s_{01}}}{\left[\|q\|^{2}+\gamma_{s_{01}} \bar{\gamma}_{s_{01}}\right]\left[\|\hat{q}\|^{2}+\gamma_{s_{01}} \bar{\gamma}_{s_{01}}\right]} \\
& =\frac{-\mathcal{A}_{01}\|\hat{q}\|\|q\|(\|q\|-\|\hat{q}\|)+\mathcal{A}_{01} \gamma_{s_{01}} \bar{\gamma}_{s_{01}}(\|q\|-\|\hat{q}\|)}{\left[\|q\|^{2}+\gamma_{s_{01}} \bar{\gamma}_{s_{01}}\right]\left[\|\hat{q}\|^{2}+\gamma_{s_{01}} \bar{\gamma}_{s_{01}}\right]} \\
& =\frac{-\mathcal{A}_{01}(\|q\|-\|\hat{q}\|)\left(\|\hat{q}\|\|q\|-\gamma_{s_{01}} \bar{\gamma}_{s_{01}}\right)}{\left[\|q\|^{2}+\gamma_{s_{01}} \bar{\gamma}_{s_{01}}\right]\left[\|\hat{q}\|^{2}+\gamma_{s_{01}} \bar{\gamma}_{s_{01}}\right]} .
\end{aligned}
$$

Also

$$
\begin{aligned}
& t_{s_{02}}-\hat{t}_{s_{02}}=\frac{-\mathcal{A}_{02}}{\|\hat{q}\|^{2}+\gamma_{s_{02}} \bar{\gamma}_{s_{02}}}-\frac{-\mathcal{A}_{02}}{\|q\|^{2}+\gamma_{s_{02}} \bar{\gamma}_{s_{02}}} \\
& =\frac{\left[\|q\|^{2}+\gamma_{s_{02}} \bar{\gamma}_{s_{02} 2}\right]\left[-\mathcal{A}_{02}\right]-\left[\|\hat{q}\|^{2}+\gamma_{s_{02}} \bar{\gamma}_{s_{02}}\right]\left[-\mathcal{A}_{02}\right]}{\left[\|q\|^{2}+\gamma_{s_{02}} \bar{\gamma}_{s_{02}}\right]\left[\|\hat{q}\|^{2}+\gamma_{s_{02}} \bar{\gamma}_{s_{02}}\right]} \\
& =\frac{-\mathcal{A}_{02}\left(\|q\|^{2}-\|\hat{q}\|^{2}\right)}{\left[\|q\|^{2}+\gamma_{s_{02}} \bar{\gamma}_{s_{02}}\right]\left[\|\hat{q}\|^{2}+\gamma_{s_{02}} \bar{\gamma}_{s_{02}}\right]} .
\end{aligned}
$$

and

$$
\begin{aligned}
& t_{s_{02}}\|q\|-\hat{t}_{s_{02}}\|\hat{q}\|=\frac{-\mathcal{A}_{02}\|\hat{q}\|}{\|\hat{q}\|^{2}+\gamma_{s_{02}} \bar{\gamma}_{s_{02}}}-\frac{-\mathcal{A}_{02}\|q\|}{\|q\|^{2}+\gamma_{s_{02}} \bar{\gamma}_{s_{02}}} \\
& =\frac{\left[\|q\|^{2}+\gamma_{s_{02}} \bar{\gamma}_{s_{02}}\right]\left[-\mathcal{A}_{02}\right]\|\hat{q}\|-\left[\|\hat{q}\|^{2}+\gamma_{s_{02}} \bar{\gamma}_{s_{02}}\right]\left[-\mathcal{A}_{02}\right]\|q\|}{\left[\|q\|^{2}+\gamma_{s_{02}} \bar{\gamma}_{s_{02}}\right]\left[\|\hat{q}\|^{2}+\gamma_{s_{02}} \bar{\gamma}_{s_{02}}\right]} \\
& =\frac{-\mathcal{A}_{02}\|\hat{q}\|\|q\|^{2}-\mathcal{A}_{02}\|\hat{q}\| \gamma_{s_{02}} \bar{\gamma}_{s_{02}}+\mathcal{A}_{02}\|q\|\|\hat{q}\|^{2}+\mathcal{A}_{02}\|q\| \gamma_{s_{02}} \bar{\gamma}_{s_{02}}}{\left[\|q\|^{2}+\gamma_{s_{02}} \bar{\gamma}_{s_{02}}\right]\left[\|\hat{q}\|^{2}+\gamma_{s_{02}} \bar{\gamma}_{s_{02}}\right]} \\
& =\frac{-\mathcal{A}_{02}\|\hat{q}\|\|q\|(\|q\|-\|\hat{q}\|)+\mathcal{A}_{02} \gamma_{s_{02}} \bar{\gamma}_{s_{02}}(\|q\|-\|\hat{q}\|)}{\left[\|q\|^{2}+\gamma_{s_{02}} \bar{\gamma}_{s_{02}}\right]\left[\|\hat{q}\|^{2}+\gamma_{s_{02}} \bar{\gamma}_{s_{02}}\right]} \\
& =\frac{-\mathcal{A}_{02}(\|q\|-\|\hat{q}\|)\left(\|\hat{q}\|\|q\|-\gamma_{s_{02}} \bar{\gamma}_{s_{02}}\right)}{\left[\|q\|^{2}+\gamma_{s_{02}} \bar{\gamma}_{s_{02}}\right]\left[\|\hat{q}\|^{2}+\gamma_{s_{02}} \bar{\gamma}_{s_{02}}\right]}
\end{aligned}
$$


Thus

$$
\begin{aligned}
& \frac{\left(\hat{t}_{s_{01}}-t_{s_{01}}\right)^{2} \gamma_{s_{01}} \bar{\gamma}_{s_{01}}+\left(t_{s_{01}}\|q\|-\hat{t}_{s_{01}}\|\hat{q}\|\right)^{2}}{\left(\hat{t}_{s_{02}}-t_{s_{02}}\right)^{2} \gamma_{s_{02}} \bar{\gamma}_{s_{02}}+\left(t_{s_{02}}\|q\|-\hat{t}_{s_{02}}\|\hat{q}\|\right)^{2}} \\
& =\frac{\left(\frac{-\mathcal{A}_{01}\left(\|q\|^{2}-\|\hat{q}\|^{2}\right)}{\left[\|q\|^{2}+\gamma_{s_{01}} \bar{\gamma}_{s_{01}}\right]\left[\|\hat{q}\|^{2}+\gamma_{s_{01}} \bar{\gamma}_{s_{01}}\right]}\right)^{2} \gamma_{s_{01}} \bar{\gamma}_{s_{01}}+\left(\frac{-\mathcal{A}_{01}(\|q\|-\|\hat{q}\|)\left(\|\hat{q}\|\|q\|-\gamma_{s_{01}} \bar{\gamma}_{s_{01}}\right)}{\left[\|q\|^{2}+\gamma_{s_{01}} \bar{\gamma}_{s_{01}}\right]\left[\|\hat{q}\|^{2}+\gamma_{s_{01}} \bar{\gamma}_{s_{01}}\right]}\right)^{2}}{\left(\frac{-\mathcal{A}_{02}\left(\|q\|^{2}-\|\hat{q}\|^{2}\right)}{\left[\|q\|^{2}+\gamma_{s_{02}} \bar{\gamma}_{s_{02}}\right]\left[\|\hat{q}\|^{2}+\gamma_{s_{02}} \bar{\gamma}_{s_{02}}\right]}\right)^{2} \gamma_{s_{02}} \bar{\gamma}_{s_{02}}+\left(\frac{-\mathcal{A}_{02}(\|q\|-\|\hat{q}\|)\left(\|\hat{q}\|\|q\|-\gamma_{s_{02} 2} \bar{s}_{s_{02}}\right)}{\left[\|q\|^{2}+\gamma_{s_{02}} \bar{\gamma}_{s_{02}}\right]\left[\|\hat{q}\|^{2}+\gamma_{s_{02}} \bar{\gamma}_{s_{02}}\right]}\right)^{2}} \\
& =\frac{\left(\frac{-\mathcal{A}_{01}(\|q\|+\|\hat{q}\|)}{\left[\|q\|^{2}+\gamma_{s_{01}} \bar{\gamma}_{s_{01}}\right]\left[\|\hat{q}\|^{2}+\gamma_{s_{01}} \bar{\gamma}_{s_{01}}\right]}\right)^{2} \gamma_{s_{01}} \bar{\gamma}_{s_{01}}+\left(\frac{-\mathcal{A}_{01}\left(\|\hat{q}\|\|q\|-\gamma_{s_{01}} \bar{\gamma}_{s_{01}}\right)}{\left[\|q\|^{2}+\gamma_{s_{01}} \bar{\gamma}_{s_{01}}\right]\left[\|\hat{q}\|^{2}+\gamma_{s_{01}} \bar{\gamma}_{s_{01}}\right]}\right)^{2}}{\left(\frac{-\mathcal{A}_{02}(\|q\|+\|\hat{q}\|)}{\left[\|q\|^{2}+\gamma_{s_{02}} \bar{\gamma}_{s_{02}}\right]\left[\|\hat{q}\|^{2}+\gamma_{s_{02}} \bar{\gamma}_{s_{02}}\right]}\right)^{2} \gamma_{s_{02}} \bar{\gamma}_{s_{02}}+\left(\frac{-\mathcal{A}_{02}\left(\|\hat{q}\|\|q\|-\gamma_{s_{02}} \bar{\gamma}_{s_{02}}\right)}{\left[\|q\|^{2}+\gamma_{s_{02}} \bar{\gamma}_{s_{02}}\right]\left[\|\hat{q}\|^{2}+\gamma_{s_{02}} \bar{\gamma}_{s_{02}}\right]}\right)^{2}} \\
& =\frac{\left([ \| q \| ^ { 2 } + \gamma _ { s _ { 0 2 } } \overline { \gamma } _ { s _ { 0 2 } } ] \left[\|\hat{q}\|^{2}+\gamma_{s_{02}} \bar{\gamma}_{\left.\left.s_{02}\right]\right)^{2}}\{\right.\right.}{\left(\left[\|q\|^{2}+\gamma_{s_{01}} \bar{\gamma}_{\left.s_{01}\right]}\right]\left[\|\hat{q}\|^{2}+\gamma_{s_{01}} \bar{\gamma}_{s_{01}}\right]\right)^{2}}\{ \\
& \frac{\left(-\mathcal{A}_{01}(\|q\|+\|\hat{q}\|)\right)^{2} \gamma_{s_{01}} \bar{\gamma}_{s_{01}}+\left(-\mathcal{A}_{01}\left(\|\hat{q}\|\|q\|-\gamma_{s_{01}} \bar{\gamma}_{s_{01}}\right)\right)^{2}}{\left(-\mathcal{A}_{02}(\|q\|+\|\hat{q}\|)\right)^{2} \gamma_{s_{02}} \bar{\gamma}_{s_{02}}+\left(-\mathcal{A}_{02}\left(\|\hat{q}\|\|q\|-\gamma_{s_{02} 2} \bar{\gamma}_{s_{02}}\right)\right)^{2}}
\end{aligned}
$$

Next we apply the limit when $\|q\| \longrightarrow \infty$ and $\|\hat{q}\| \longrightarrow \infty$,

$$
\begin{aligned}
& \lim _{\substack{\|q\| \longrightarrow \infty \\
\|\hat{q}\| \longrightarrow \infty}} \frac{\left(\left[\|q\|^{2}+\gamma_{s_{02}} \bar{\gamma}_{s_{02}}\right]\left[\|\hat{q}\|^{2}+\gamma_{s_{02}} \bar{\gamma}_{s_{02}}\right]\right)^{2}}{\left(\left[\|q\|^{2}+\gamma_{s_{01}} \bar{\gamma}_{s_{01}}\right]\left[\|\hat{q}\|^{2}+\gamma_{s_{01}} \bar{\gamma}_{s_{01}}\right]\right)^{2}}\{ \\
& \left.\frac{\left(-\mathcal{A}_{01}(\|q\|+\|\hat{q}\|)\right)^{2} \gamma_{s_{01}} \bar{\gamma}_{s_{01}}+\left(-\mathcal{A}_{01}\left(\|\hat{q}\|\|q\|-\gamma_{s_{01}} \bar{\gamma}_{s_{01}}\right)\right)^{2}}{\left(-\mathcal{A}_{02}(\|q\|+\|\hat{q}\|)\right)^{2} \gamma_{s_{02}} \bar{\gamma}_{s_{02}}+\left(-\mathcal{A}_{02}\left(\|\hat{q}\|\|q\|-\gamma_{s_{02}} \bar{\gamma}_{s_{02}}\right)\right)^{2}}\right\} \\
& =\frac{\mathcal{A}_{0}^{2}}{4 \mathcal{D}_{0}^{2}}>0
\end{aligned}
$$


Also we apply the limit when $\|q\| \longrightarrow 0$ and $\|\hat{q}\| \longrightarrow 0$,

$$
\begin{aligned}
& \lim _{\substack{\|q\| \rightarrow 0 \\
\|\hat{q}\| \longrightarrow 0}} \frac{\left(\left[\|q\|^{2}+\gamma_{s_{02}} \bar{\gamma}_{s_{02}}\right]\left[\|\hat{q}\|^{2}+\gamma_{s_{02}} \bar{\gamma}_{s_{02}}\right]\right)^{2}}{\left(\left[\|q\|^{2}+\gamma_{s_{01}} \bar{\gamma}_{s_{01}}\right]\left[\|\hat{q}\|^{2}+\gamma_{s_{01}} \bar{\gamma}_{s_{01}}\right]\right)^{2}}\{ \\
& \left.\frac{\left(-\mathcal{A}_{01}(\|q\|+\|\hat{q}\|)\right)^{2} \gamma_{s_{01}} \bar{\gamma}_{s_{01}}+\left(-\mathcal{A}_{01}\left(\|\hat{q}\|\|q\|-\gamma_{s_{01}} \bar{\gamma}_{s_{01}}\right)\right)^{2}}{\left(-\mathcal{A}_{02}(\|q\|+\|\hat{q}\|)\right)^{2} \gamma_{s_{02}} \bar{\gamma}_{s_{02}}+\left(-\mathcal{A}_{02}\left(\|\hat{q}\|\|q\|-\gamma_{s_{02}} \bar{\gamma}_{s_{02}}\right)\right)^{2}}\right\} \\
& =\frac{\left(\gamma_{s_{02}} \bar{\gamma}_{s_{02}}\right)^{4}}{\left(\gamma_{s_{01}} \bar{\gamma}_{s_{01}}\right)^{4}}\left\{\frac{\left(-\mathcal{A}_{01} \gamma_{s_{01}} \bar{\gamma}_{s_{01}}\right)^{2}}{\left(-\mathcal{A}_{02} \gamma_{s_{02}} \bar{\gamma}_{s_{02}}\right)^{2}}\right\}=\frac{\left(\mathcal{A}_{01} \gamma_{s_{02}} \bar{\gamma}_{s_{02}}\right)^{2}}{\left(\mathcal{A}_{02} \gamma_{s_{01}} \bar{\gamma}_{s_{02}}\right)^{2}}>0
\end{aligned}
$$

Moreover, the denominator of

$$
\frac{\left(\hat{t}_{s_{01}}-t_{s_{01}}\right)^{2} \gamma_{s_{01}} \bar{\gamma}_{s_{01}}+\left(t_{s_{01}}\|q\|-\hat{t}_{s_{01}}\|\hat{q}\|\right)^{2}}{\left(\hat{t}_{s_{02}}-t_{s_{02}}\right)^{2} \gamma_{s_{02}} \bar{\gamma}_{s_{02}}+\left(t_{s_{02}}\|q\|-\hat{t}_{s_{02}}\|\hat{q}\|\right)^{2}}
$$

does not equal zero for all $\|q\|$ and $\|\hat{q}\|$, hence the quantity is a continuous function on a closed, bounded set in $\mathbb{R}^{2}$, then it attains an absolute maximum value at some points in this set. The proof is complete.

Theorem 5.9.3 any two extended sphere metric with $\alpha_{s}=0$ are equivalent.

Proof By proposition 5.9.1, lemma 5.9.2 and theorem 5.2.2, the result follow.

Corollary 5.9.4 Any two distinct extended ellipsoid metrics when $\alpha_{e}=0$ are equivalent.

\subsection{Equivalence Between Extended Sphere Metric when $\alpha_{s} \neq 0$ And An Extended Cone Metric}

As another example of equivalence we will show the equivalence between an extended cone metric and an extended sphere metric with $\alpha_{s} \neq 0$. We start with the following proposition.

Proposition 5.10.1 Let $t_{s}$ and $t_{c}$ subject to (3.5.17) and (3.7.16) respectively, then we have the quantity $\frac{t_{s}}{t_{c}}$ is bounded above and below. 
Proof Compute the ratio between $t_{s}$ and $t_{c}$

$$
\begin{aligned}
\frac{t_{s}}{t_{c}}= & \frac{\frac{-\alpha_{s}}{\sqrt{F}+\frac{\mathcal{A}}{2}}}{\frac{\alpha_{c}}{\|q\|+\frac{\left|\gamma_{c}\right|}{m}}}=\frac{-\alpha_{s}\left(\|q\|+\frac{\left|\gamma_{c}\right|}{m}\right)}{\alpha_{c}\left(\frac{\mathcal{A}}{2}+\sqrt{\frac{\mathcal{A}^{2}}{4}-\left(\|q\|^{2}+\gamma_{s} \bar{\gamma}_{s}\right) \alpha_{s}}\right)} \\
= & \frac{-\alpha_{s}\|q\|\left(1+\frac{\left|\gamma_{c}\right|}{m\|q\|}\right)}{\alpha_{c}\|q\|\left(\frac{\mathcal{A}}{2\|q\|}+\sqrt{\frac{\mathcal{A}^{2}}{4\|q\|^{2}}-\left(1+\frac{\gamma_{s} \bar{\gamma}_{s}}{\|q\|^{2}}\right) \alpha_{s}}\right)} \\
= & \frac{-\alpha_{s}\left(1+\frac{\left|\gamma_{c}\right|}{m\|q\|}\right)}{\alpha_{c}\left(\frac{\mathcal{A}}{2\|q\|}+\sqrt{\frac{\mathcal{A}^{2}}{4\|q\|^{2}}-\left(1+\frac{\gamma_{s} \bar{\gamma}_{s}}{\|q\|^{2}}\right) \alpha_{s}}\right)}
\end{aligned}
$$

Compute the limit of $\frac{t_{s}}{t_{c}}$ as $\|q\| \longrightarrow 0$

$$
\begin{aligned}
\lim _{\|q\| \longrightarrow 0} \frac{t_{s}}{t_{c}} & =\lim _{\|q\| \rightarrow 0} \frac{-\alpha_{s}\left(\|q\|+\frac{\left|\gamma_{c}\right|}{m}\right)}{\alpha_{c}\left(\frac{\mathcal{A}}{2}+\sqrt{\frac{\mathcal{A}^{2}}{4}-\left(\|q\|^{2}+\gamma_{s} \bar{\gamma}_{s}\right) \alpha_{s}}\right)} \\
& =\frac{-\alpha_{s} \frac{\left|\gamma_{c}\right|}{m}}{\alpha_{c}\left(\frac{\mathcal{A}}{2}+\sqrt{\frac{\mathcal{A}^{2}}{4}-\gamma_{s} \bar{\gamma}_{s} \alpha_{s}}\right)} \neq 0
\end{aligned}
$$

And compute the limit of $\frac{t_{s}}{t_{c}}$ as $\|q\| \longrightarrow \infty$

$$
\begin{aligned}
\lim _{\|q\| \rightarrow \infty} \frac{t_{s}}{t_{c}} & =\lim _{\|q\| \rightarrow \infty} \frac{-\alpha_{s}\left(1+\frac{\left|\gamma_{c}\right|}{m\|q\|}\right)}{\alpha_{c}\left(\frac{\mathcal{A}}{2\|q\|}+\sqrt{\frac{\mathcal{A}^{2}}{4\|q\|^{2}}-\left(1+\frac{\gamma_{s} \bar{\gamma}_{s}}{\|q\|^{2}}\right) \alpha_{s}}\right)} \\
& =\frac{-\alpha_{s}}{\alpha_{c} \sqrt{-\alpha_{s}}}=\frac{\sqrt{-\alpha_{s}}}{\alpha_{c}} \neq 0
\end{aligned}
$$

Moreover, the denominator of $\frac{t_{s}}{t_{c}}$ does not equal zero for all $\|q\|$, also, the numerator and the denominator are positive and bounded on $[\hat{\rho}, \tilde{\rho}]$. Hence the quotient is a continuous function of $\|q\|$ on the closed interval $[\hat{\rho}, \tilde{\rho}]$, this implies that the quotient has a positive minimum and maximum on $[\hat{\rho}, \tilde{\rho}]$. The proof is complete. 
Theorem 5.10.2 Any extended sphere metric with $\alpha_{s} \neq 0$ is equivalent to any extended cone metric.

Proof We first determine various expressions for $\hat{t}_{s}-t_{s}$ and $\hat{t}_{s}\|\hat{q}\|-t_{s}\|q\|$ that will be shown to be useful in the sequel.

$$
\begin{aligned}
\hat{t}_{s}-t_{s}= & \frac{-\alpha_{s}}{\sqrt{\hat{F}}+\frac{\mathcal{A}}{2}}-\frac{-\alpha_{s}}{\sqrt{F}+\frac{\mathcal{A}}{2}}=\frac{-\alpha_{s}\left(\sqrt{F}+\frac{\mathcal{A}}{2}\right)+\alpha_{s}\left(\sqrt{\hat{F}}+\frac{\mathcal{A}}{2}\right)}{\left(\sqrt{\hat{F}}+\frac{\mathcal{A}}{2}\right)\left(\sqrt{F}+\frac{\mathcal{A}}{2}\right)} \\
& =\frac{-\alpha_{s} \sqrt{F}+\alpha_{s} \sqrt{\hat{F}}}{\left(\sqrt{\hat{F}}+\frac{\mathcal{A}}{2}\right)\left(\sqrt{F}+\frac{\mathcal{A}}{2}\right)}=\frac{-\alpha_{s}(\sqrt{F}-\sqrt{\hat{F}})}{\left(\sqrt{\hat{F}}+\frac{\mathcal{A}}{2}\right)\left(\sqrt{F}+\frac{\mathcal{A}}{2}\right)} \\
& =\frac{-\alpha_{s}(F-\hat{F})}{\left(\sqrt{\hat{F}}+\frac{\mathcal{A}}{2}\right)\left(\sqrt{F}+\frac{\mathcal{A}}{2}\right)(\sqrt{F}+\sqrt{\hat{F}})} \\
& =\frac{\alpha_{s}^{2}\left(\|q\|^{2}-\|\hat{q}\|^{2}\right)}{\left(\sqrt{\hat{F}}+\frac{\mathcal{A}}{2}\right)\left(\sqrt{F}+\frac{\mathcal{A}}{2}\right)(\sqrt{F}+\sqrt{\hat{F}})}
\end{aligned}
$$

Also

$$
\begin{aligned}
& t_{s}\|q\|-\hat{t}_{s}\|\hat{q}\|=\frac{-\alpha_{s}\|q\|}{\sqrt{F}+\frac{\mathcal{A}}{2}}-\frac{-\alpha_{s}\|\hat{q}\|}{\sqrt{\hat{F}}+\frac{\mathcal{A}}{2}} \\
&=\frac{-\alpha_{s}\|q\|\left(\sqrt{\hat{F}}+\frac{\mathcal{A}}{2}\right)+\alpha_{s}\|\hat{q}\|\left(\sqrt{F}+\frac{\mathcal{A}}{2}\right)}{\left(\sqrt{\hat{F}}+\frac{\mathcal{A}}{2}\right)\left(\sqrt{F}+\frac{\mathcal{A}}{2}\right)} \\
&=\frac{-\alpha_{s} \frac{\mathcal{A}}{2}(\|q\|-\|\hat{q}\|)+\left(-\alpha_{s}(\|q\| \sqrt{\hat{F}}-\|\hat{q}\| \sqrt{F})\right)}{\left(\sqrt{\hat{F}}+\frac{\mathcal{A}}{2}\right)\left(\sqrt{F}+\frac{\mathcal{A}}{2}\right)} \\
&= \frac{-\alpha_{s} \frac{\mathcal{A}}{2}(\|q\|-\|\hat{q}\|)(\|q\| \sqrt{\hat{F}}+\|\hat{q}\| \sqrt{F})+\left(-\alpha_{s}\left(\|q\|^{2} \hat{F}-\|\hat{q}\|^{2} F\right)\right)}{\left(\sqrt{\hat{F}}+\frac{\mathcal{A}}{2}\right)\left(\sqrt{F}+\frac{\mathcal{A}}{2}\right)(\|q\| \sqrt{\hat{F}}+\|\hat{q}\| \sqrt{F})} \\
&=\frac{-\alpha_{s} \frac{\mathcal{A}}{2}(\|q\|-\|\hat{q}\|)(\|q\| \sqrt{\hat{F}}+\|\hat{q}\| \sqrt{F})+\left(-\alpha_{s}\left(\frac{\mathcal{A}^{2}}{4}-\gamma_{s} \bar{\gamma}_{s} \alpha_{s}\right)\left(\|q\|^{2}-\|\hat{q}\|^{2}\right)\right)}{\left(\sqrt{\hat{F}}+\frac{\mathcal{A}}{2}\right)\left(\sqrt{F}+\frac{\mathcal{A}}{2}\right)(\|q\| \sqrt{\hat{F}}+\|\hat{q}\| \sqrt{F})}
\end{aligned}
$$




\section{Therefore}

$$
\begin{aligned}
& \frac{\left(\hat{t}_{s}-t_{s}\right)^{2} \gamma_{s} \bar{\gamma}_{s}+\left(t_{s}\|q\|-\hat{t}_{s}\|\hat{q}\|\right)^{2}}{\left(\hat{t}_{c}-t_{c}\right)^{2} \gamma_{c} \bar{\gamma}_{c}+\left(t_{c}\|q\|-\hat{t}_{c}\|\hat{q}\|\right)^{2}}=\frac{\left(\hat{t}_{s}-t_{s}\right)^{2} \gamma_{s} \bar{\gamma}_{s}+\left(t_{s}\|q\|-\hat{t}_{s}\|\hat{q}\|\right)^{2}}{\left(\hat{t}_{c}-t_{c}\right)^{2} \gamma_{c} \bar{\gamma}_{c}+\frac{\left|\gamma_{c}\right|^{2}}{m^{2}}\left(\hat{t}_{c}-t_{c}\right)^{2}} \\
& =\frac{\left(\hat{t}_{s}-t_{s}\right)^{2} \gamma_{s} \bar{\gamma}_{s}+\left(t_{s}\|q\|-\hat{t}_{s}\|\hat{q}\|\right)^{2}}{\left(\hat{t}_{c}-t_{c}\right)^{2}\left(\gamma_{c} \bar{\gamma}_{c}+\frac{\left|\gamma_{c}\right|^{2}}{m^{2}}\right)}=\frac{\left(\hat{t}_{s}-t_{s}\right)^{2} \gamma_{s} \bar{\gamma}_{s}+\left(t_{s}\|q\|-\hat{t}_{s}\|\hat{q}\|\right)^{2}}{\left(\frac{\alpha_{c}(\|q\|-\|\hat{q}\|)}{\left(\|\hat{q}\|+\frac{|\gamma c|}{m}\right)\left(\|q\|+\frac{\left|\gamma_{c}\right|}{m}\right)}\right)^{2}\left(\gamma_{c} \bar{\gamma}_{c}+\frac{\mid \gamma_{c}{ }^{2}}{m^{2}}\right)} \\
& =\frac{\left(\frac{\alpha_{s}^{2}\left(\|q\|^{2}-\|\hat{q}\|^{2}\right.}{\left(\sqrt{\hat{F}}+\frac{\mathcal{A}}{2}\right)\left(\sqrt{F}+\frac{\mathcal{A}}{2}\right)(\sqrt{F}+\sqrt{\hat{F}})}\right)^{2} \gamma_{s} \bar{\gamma}_{s}}{\left(\frac{\alpha_{c}(\|q\|-\|\hat{q}\|)}{\left(\|\hat{q}\|+\frac{\left.\mid \gamma_{c}\right)}{m}\right)\left(\|q\|+\frac{\left|\gamma_{c}\right|}{m}\right)}\right)^{2}\left(\gamma_{c} \bar{\gamma}_{c}+\frac{\left|\gamma_{c}\right|^{2}}{m^{2}}\right)} \\
& +\frac{\left(\frac{-\alpha_{s} \frac{\mathcal{A}}{2}(\|q\|-\|\hat{q}\|)(\|q\| \sqrt{\hat{F}}+\|\hat{q}\| \sqrt{F})+\left(-\alpha_{s}\left(\frac{\mathcal{A}^{2}}{4}-\gamma_{s} \bar{\gamma}_{s} \alpha_{s}\right)\left(\|q\|^{2}-\|\hat{q}\|^{2}\right)\right)}{\left(\sqrt{\hat{F}}+\frac{\mathcal{A}}{2}\right)\left(\sqrt{F}+\frac{\mathcal{A}}{2}\right)(\|q\| \sqrt{\hat{F}}+\|\hat{q}\| \sqrt{F})}\right)^{2}}{\left(\frac{\alpha_{c}(\|q\|-\|\hat{q}\|)}{\left(\|\hat{q}\|+\frac{\gamma_{c} c}{m}\right)\left(\|q\|+\frac{\left|\gamma_{c}\right|}{m}\right)}\right)^{2}\left(\gamma_{c} \bar{\gamma}_{c}+\frac{\left|\gamma_{c}\right|^{2}}{m^{2}}\right)} \\
& =\frac{\left(\frac{\alpha_{s}^{2}(\|q\|+\|\hat{q}\|)}{\left(\sqrt{\hat{F}}+\frac{\mathcal{A}}{2}\right)\left(\sqrt{F}+\frac{\mathcal{A}}{2}\right)(\sqrt{F}+\sqrt{\hat{F}})}\right)^{2} \gamma_{s} \bar{\gamma}_{s}}{\left(\frac{\alpha_{c}}{\left(\|\hat{q}\|+\frac{\left|\gamma_{c}\right|}{m}\right)\left(\|q\|+\frac{\left|\gamma_{c}\right|}{m}\right)}\right)^{2}\left(\gamma_{c} \bar{\gamma}_{c}+\frac{\left|\gamma_{c}\right|^{2}}{m^{2}}\right)} \\
& +\frac{\left(\frac{-\alpha_{s} \frac{\mathcal{A}}{2}(\|q\| \sqrt{\hat{F}}+\|\hat{q}\| \sqrt{F})+\left(-\alpha_{s}\left(\frac{\mathcal{A}^{2}}{4}-\gamma_{s} \bar{\gamma}_{s} \alpha_{s}\right)(\|q\|+\|\hat{q}\|)\right)}{\left(\sqrt{\hat{F}}+\frac{\mathcal{A}}{2}\right)\left(\sqrt{F}+\frac{\mathcal{A}}{2}\right)(\|q\| \sqrt{\hat{F}}+\|\hat{q}\| \sqrt{F})}\right)^{2}}{\left(\frac{\alpha_{c}}{\left(\|\hat{q}\|+\frac{\left|\gamma_{c}\right|}{m}\right)\left(\|q\|+\frac{\left|\gamma_{c}\right|}{m}\right)}\right)^{2}\left(\gamma_{c} \bar{\gamma}_{c}+\frac{\left|\gamma_{c}\right|^{2}}{m^{2}}\right)}
\end{aligned}
$$




$$
\begin{aligned}
& =\frac{\left(\|\hat{q}\|+\frac{\left|\gamma_{c}\right|}{m}\right)^{2}\left(\|q\|+\frac{\left|\gamma_{c}\right|}{m}\right)^{2}\left(\alpha_{s}^{2}(\|q\|+\|\hat{q}\|)\right)^{2} \gamma_{s} \bar{\gamma}_{s}}{\left(\alpha_{c}\left(\sqrt{\hat{F}}+\frac{\mathcal{A}}{2}\right)\left(\sqrt{F}+\frac{\mathcal{A}}{2}\right)(\sqrt{F}+\sqrt{\hat{F}})\right)^{2}\left(\gamma_{c} \bar{\gamma}_{c}+\frac{\left|\gamma_{c}\right|^{2}}{m^{2}}\right)} \\
& +\frac{\left(\|\hat{q}\|+\frac{\left|\gamma_{c}\right|}{m}\right)^{2}\left(\|q\|+\frac{\left|\gamma_{c}\right|}{m}\right)^{2}}{\left(\alpha_{c}\left(\sqrt{\hat{F}}+\frac{\mathcal{A}}{2}\right)\left(\sqrt{F}+\frac{\mathcal{A}}{2}\right)\right)^{2}\left(\gamma_{c} \bar{\gamma}_{c}+\frac{\left|\gamma_{c}\right|^{2}}{m^{2}}\right)}\{ \\
& \left.\frac{\left(-\alpha_{s} \frac{\mathcal{A}}{2}(\|q\| \sqrt{\hat{F}}+\|\hat{q}\| \sqrt{F})+\left(-\alpha_{s}\left(\frac{\mathcal{A}^{2}}{4}-\gamma_{s} \bar{\gamma}_{s} \alpha_{s}\right)(\|q\|+\|\hat{q}\|)\right)\right)^{2}}{(\|q\| \sqrt{\hat{F}}+\|\hat{q}\| \sqrt{F})^{2}}\right\}
\end{aligned}
$$

By lemmas 5.2.3, 5.2.4 we have the quantity

$$
\frac{\left(\|\hat{q}\|+\frac{\left|\gamma_{c}\right|}{m}\right)^{2}\left(\|q\|+\frac{\left|\gamma_{c}\right|}{m}\right)^{2}\left(\alpha_{s}^{2}(\|q\|+\|\hat{q}\|)\right)^{2} \gamma_{s} \bar{\gamma}_{s}}{\left(\alpha_{c}\left(\sqrt{\hat{F}}+\frac{\mathcal{A}}{2}\right)\left(\sqrt{F}+\frac{\mathcal{A}}{2}\right)(\sqrt{F}+\sqrt{\hat{F}})\right)^{2}\left(\gamma_{c} \bar{\gamma}_{c}+\frac{\left|\gamma_{c}\right|^{2}}{m^{2}}\right)}
$$

is bounded above and below. And by 5.2.6, the quantity

$$
\begin{aligned}
& +\frac{\left(\|\hat{q}\|+\frac{\left|\gamma_{c}\right|}{m}\right)^{2}\left(\|q\|+\frac{\left|\gamma_{c}\right|}{m}\right)^{2}}{\left(\alpha_{c}\left(\sqrt{\hat{F}}+\frac{\mathcal{A}}{2}\right)\left(\sqrt{F}+\frac{\mathcal{A}}{2}\right)\right)^{2}\left(\gamma_{c} \bar{\gamma}_{c}+\frac{\left|\gamma_{c}\right|^{2}}{m^{2}}\right)}\{ \\
& \left.\frac{\left(-\alpha_{s} \frac{\mathcal{A}}{2}(\|q\| \sqrt{\hat{F}}+\|\hat{q}\| \sqrt{F})+\left(-\alpha_{s}\left(\frac{\mathcal{A}^{2}}{4}-\gamma_{s} \bar{\gamma}_{s} \alpha_{s}\right)(\|q\|+\|\hat{q}\|)\right)\right)^{2}}{(\|q\| \sqrt{\hat{F}}+\|\hat{q}\| \sqrt{F})^{2}}\right\}
\end{aligned}
$$

is bounded above but not below, note that $F=\frac{\mathcal{A}^{2}}{4}-\left(\|q\|^{2}+\gamma_{s} \bar{\gamma}_{s}\right) \alpha_{s}$, $\hat{F}=\frac{\mathcal{A}^{2}}{4}-\left(\|\hat{q}\|^{2}+\gamma_{s} \bar{\gamma}_{s}\right) \alpha_{s} \delta_{1}=\frac{\left|\gamma_{c}\right|}{m}>0, \delta_{2}=\frac{\mathcal{A}}{2}, \delta_{3}=\gamma_{s} \bar{\gamma}_{s}, \delta_{4}=-\alpha_{s}>0$, $\delta_{5}=-\alpha_{s}\left(\frac{\mathcal{A}^{2}}{4}-\gamma_{s} \bar{\gamma}_{s} \alpha_{s}\right)>0$ and $\delta_{6}=-\alpha_{s} \frac{\mathcal{A}}{2}<0$. Also $\delta_{2}^{2}+\delta_{3} \delta_{4}>0$ and $\delta_{6}+\frac{\delta_{5}}{\sqrt{\delta_{2}^{2}+\delta_{3} \delta_{4}}} \geq 0$. The result follow.

Corollary 5.10.3 Any extended ellipsoid metric with $\alpha_{e} \neq 0$ is equivalent to any extended cone metric. 


\subsection{Nonequivalence Between An Extended Sphere Metric when $\alpha_{s}=0$ and An Ex- tended Cone Metric}

Theorem 5.11.1 Any extended sphere metric with $\alpha_{s}=0$ is nonequivalent to any extended cone metric.

Proof We compute the quotient between an extended sphere metric with $\alpha_{s}=0$ and an extended cone metric as

$$
\frac{\left(\hat{t}_{s_{0}}(\|\hat{q}\|)-t_{s_{0}}(\|q\|)\right)^{2} \gamma_{s_{0}} \bar{\gamma}_{s_{0}}+\left\|t_{s_{0}}(\|q\|) q-\hat{t}_{s_{0}}(\|\hat{q}\|) \hat{q}\right\|^{2}}{\left(\hat{t}_{c}(\|\hat{q}\|)-t_{c}(\|q\|)\right)^{2} \gamma_{c} \bar{\gamma}_{c}+\left\|t_{c}(\|q\|) q-\hat{t}_{c}(\|\hat{q}\|) \hat{q}\right\|^{2}}
$$

Let $\|q\| \longrightarrow \infty$ and $0<\|\hat{q}\|<\infty$ then we have

$$
t_{s_{0}}(\|q\|) \longrightarrow 0 \text { and } t_{c}(\|q\|) \longrightarrow 0
$$

Then we are left with the quotient

$$
\frac{\left(\hat{t}_{s_{0}}(\|\hat{q}\|)\right)^{2} \gamma_{s_{0}} \bar{\gamma}_{s_{0}}+\left\|\hat{t}_{s_{0}}(\|\hat{q}\|) \hat{q}\right\|^{2}}{\left(\hat{t}_{c}(\|\hat{q}\|)\right)^{2} \gamma_{c} \bar{\gamma}_{c}+\left\|\hat{t}_{c}(\|\hat{q}\|) \hat{q}\right\|^{2}}=\frac{\left(\hat{t}_{s_{0}}(\|\hat{q}\|)\right)^{2}\left(\gamma_{s_{0}} \bar{\gamma}_{s_{0}}+\|\hat{q}\|^{2}\right)}{\left(\hat{t}_{c}(\|\hat{q}\|)\right)^{2}\left(\gamma_{c} \bar{\gamma}_{c}+\|\hat{q}\|^{2}\right)}
$$

The quantity

$$
\frac{\gamma_{s_{0}} \bar{\gamma}_{s_{0}}+\|\hat{q}\|^{2}}{\gamma_{c} \bar{\gamma}_{c}+\|\hat{q}\|^{2}}
$$

is a Möbius transformation if we put $z=\|\hat{q}\|^{2}$. Consequently, it is bounded above and below. Now let $\|\hat{q}\| \longrightarrow \infty$ then we have

$$
\frac{\left(\hat{t}_{s_{0}}(\|\hat{q}\|)\right)^{2}}{\left(\hat{t}_{c}(\|\hat{q}\|)\right)^{2}} \longrightarrow 0
$$

Thus the extended sphere metric with $\alpha_{s}=0$ and the extended cone metric cannot be equivalent.

Corollary 5.11.2 any extended ellipsoid metric with $\alpha_{e}=0$ is nonequivalent to any extended cone metric. 


\section{Chapter 6}

\section{Equivalence and \\ Nonequivalence Between An \\ Extended Metric and A Projection Metric}

In this chapter we will introduce important theorems about the relation between an extended metric and a projection metric. It is important to determine whether or not a general metric extended is equivalent to its projection. this is the subject of our next theorems. It is intriguing to discover that such metrics are not necessarily equivalent.

\subsection{General Theorems}

Theorem 6.1.1 Assume

$$
\left|\frac{\hat{t}_{g}-t_{g}}{t_{g}\|q\|-\hat{t}_{g}\|\hat{q}\|}\right| \leq \mathcal{M}<\infty
$$

where $\mathcal{M}$ is a positive real number, then an extended general metric is equivalent to its projection metric.

Proof Consider an extended general metric

$$
\begin{aligned}
& D_{g}^{2}\{(\overrightarrow{0}, q),(\overrightarrow{0}, \hat{q})\} \\
& =\left(\hat{t}_{g}-t_{g}\right)^{2} \gamma_{g} \bar{\gamma}_{g}+\left(t_{g}\|q\|-\hat{t}_{g}\|\hat{q}\|\right)^{2}+\hat{t}_{g} t_{g}(2\|q\|\|\hat{q}\|-\langle\hat{q}, q\rangle-\langle q, \hat{q}\rangle)
\end{aligned}
$$


with it is a projection metric

$$
D_{g}^{2}\{(\overrightarrow{0}, q),(\overrightarrow{0}, \hat{q})\}=\left(t_{g}\|q\|-\hat{t}_{g}\|\hat{q}\|\right)^{2}+\hat{t}_{g} t_{g}(2\|q\|\|\hat{q}\|-\langle\hat{q}, q\rangle-\langle q, \hat{q}\rangle)
$$

then

$$
\frac{\left(\hat{t}_{g}-t_{g}\right)^{2} \gamma_{g} \bar{\gamma}_{g}+\left(t_{g}\|q\|-\hat{t}_{g}\|\hat{q}\|\right)^{2}+\hat{t}_{g} t_{g}(2\|q\|\|\hat{q}\|-\langle\hat{q}, q\rangle-\langle q, \hat{q}\rangle)}{\left(t_{g}\|q\|-\hat{t}_{g}\|\hat{q}\|\right)^{2}+\hat{t}_{g} t_{g}(2\|q\|\|\hat{q}\|-\langle\hat{q}, q\rangle-\langle q, \hat{q}\rangle)}
$$

Clearly the quantity in (6.1.2) is greater than one thus it is bounded below. By our assumption (6.1.1) we have

$$
\begin{aligned}
& \frac{\left(\hat{t}_{g}-t_{g}\right)^{2} \gamma_{g} \bar{\gamma}_{g}+\left(t_{g}\|q\|-\hat{t}_{g}\|\hat{q}\|\right)^{2}+\hat{t}_{g} t_{g}(2\|q\|\|\hat{q}\|-\langle\hat{q}, q\rangle-\langle q, \hat{q}\rangle)}{\left(t_{g}\|q\|-\hat{t}_{g}\|\hat{q}\|\right)^{2}+\hat{t}_{g} t_{g}(2\|q\|\|\hat{q}\|-\langle\hat{q}, q\rangle-\langle q, \hat{q}\rangle)} \leq \\
& \frac{\mathcal{M}^{2}\left(t_{g}\|q\|-\hat{t}_{g}\|\hat{q}\|\right)^{2} \gamma_{g} \bar{\gamma}_{g}+\left(t_{g}\|q\|-\hat{t}_{g}\|\hat{q}\|\right)^{2}+\hat{t}_{g} t_{g}(2\|q\|\|\hat{q}\|-\langle\hat{q}, q\rangle-\langle q, \hat{q}\rangle)}{\left(t_{g}\|q\|-\hat{t}_{g}\|\hat{q}\|\right)^{2}+\hat{t}_{g} t_{g}(2\|q\|\|\hat{q}\|-\langle\hat{q}, q\rangle-\langle q, \hat{q}\rangle)} \\
& =\frac{\left(\mathcal{M}^{2} \gamma_{g} \bar{\gamma}_{g}+1\right)^{2}\left(t_{g}\|q\|-\hat{t}_{g}\|\hat{q}\|\right)^{2}+\hat{t}_{g} t_{g}(2\|q\|\|\hat{q}\|-\langle\hat{q}, q\rangle-\langle q, \hat{q}\rangle)}{\left(t_{g}\|q\|-\hat{t}_{g}\|\hat{q}\|\right)^{2}+\hat{t}_{g} t_{g}(2\|q\|\|\hat{q}\|-\langle\hat{q}, q\rangle-\langle q, \hat{q}\rangle)} \\
& \leq \frac{\left(\mathcal{M}^{2} \gamma_{g} \bar{\gamma}_{g}+1\right)^{2}\left(\left(t_{g}\|q\|-\hat{t}_{g}\|\hat{q}\|\right)^{2}+\hat{t}_{g} t_{g}(2\|q\|\|\hat{q}\|-\langle\hat{q}, q\rangle-\langle q, \hat{q}\rangle)\right)}{\left(t_{g}\|q\|-\hat{t}_{g}\|\hat{q}\|\right)^{2}+\hat{t}_{g} t_{g}(2\|q\|\|\hat{q}\|-\langle\hat{q}, q\rangle-\langle q, \hat{q}\rangle)} \\
& =\left(\mathcal{M}^{2} \gamma_{g} \bar{\gamma}_{g}+1\right)^{2}
\end{aligned}
$$

therefore the quantity in (6.1.2) is bounded above. The proof is complete.

Theorem 6.1.2 Assume

$$
\left|\frac{\hat{t}_{g}-t_{g}}{t_{g}\|q\|-\hat{t}_{g}\|\hat{q}\|}\right| \longrightarrow \infty
$$

when

$$
2\|q\|\|\hat{q}\|-\langle\hat{q}, q\rangle-\langle q, \hat{q}\rangle=0
$$

as $\|q\|$ tends to a certain value. Then an extended general metric is nonequivalent to its projection metric. 
Proof Assume $\hat{q}=w q$ where $w>0 w \neq 1$ then

$$
\begin{aligned}
& 2\|q\|\|\hat{q}\|-\langle\hat{q}, q\rangle-\langle q, \hat{q}\rangle=2\|q\|\|w q\|-\langle w q, q\rangle-\langle q, w q\rangle \\
& =2 w\|q\|^{2}-2 w\langle q, q\rangle=2 w\|q\|^{2}-2 w\|q\|^{2}=0
\end{aligned}
$$

therefore

$$
\begin{aligned}
& \frac{\left(\hat{t}_{g}-t_{g}\right)^{2} \gamma_{g} \bar{\gamma}_{g}+\left(t_{g}\|q\|-\hat{t}_{g}\|\hat{q}\|\right)^{2}+\hat{t}_{g} t_{g}(2\|q\|\|\hat{q}\|-\langle\hat{q}, q\rangle-\langle q, \hat{q}\rangle)}{\left(t_{g}\|q\|-\hat{t}_{g}\|\hat{q}\|\right)^{2}+\hat{t}_{g} t_{g}(2\|q\|\|\hat{q}\|-\langle\hat{q}, q\rangle-\langle q, \hat{q}\rangle)} \\
& =\frac{\left(\hat{t}_{g}-t_{g}\right)^{2} \gamma_{g} \bar{\gamma}_{g}+\left(t_{g}\|q\|-\hat{t}_{g}\|\hat{q}\|\right)^{2}}{\left(t_{g}\|q\|-\hat{t}_{g}\|\hat{q}\|\right)^{2}}=\frac{\left(\hat{t}_{g}-t_{g}\right)^{2} \gamma_{g} \bar{\gamma}_{g}}{\left(t_{g}\|q\|-\hat{t}_{g}\|\hat{q}\|\right)^{2}}+1
\end{aligned}
$$

By our assumption the extended general metric is not equivalent to its projection metric. The proof is complete.

\subsection{Equivalence Among Any Extended and Any Projection Cone Metric , Any Paraboloid Metric and Any Hyperboloid Metric.}

Theorem 6.2.1 Any extended cone metric is equivalent to its projection metric.

Proof Since the quantity

$$
\frac{\hat{t}_{c}-t_{c}}{t_{c}\|q\|-\hat{t}_{c}\|\hat{q}\|}=\frac{\hat{t}_{c}-t_{c}}{\frac{\left|\gamma_{c}\right|}{m}\left(\hat{t}_{c}-t_{c}\right)}=\frac{1}{\frac{\left|\gamma_{c}\right|}{m}}=\frac{m}{\left|\gamma_{c}\right|}
$$

is bounded above and below, therefore by theorem 6.1.1 the extended cone metric is equivalent to its a projection metric. The proof is complete.

Theorem 6.2.2 Any projection paraboloid metric is equivalent to any projection cone metric. Moreover any extended paraboloid metric is equivalent to its projection metric.

Proof We have shown before that

$$
\begin{aligned}
& \frac{\hat{t}_{p}\|\hat{q}\|-t_{p}\|q\|}{\hat{t}_{c}\|\hat{q}\|-t_{c}\|q\|} \\
& =\frac{-2 \alpha_{p}(\|q\| \sqrt{\hat{G}}+\|\hat{q}\| \sqrt{G}+\|q\|+\|\hat{q}\|)\left(\|q\|+\frac{\left|\gamma_{c}\right|}{m}\right)\left(\|\hat{q}\|+\frac{\left|\gamma_{c}\right|}{m}\right)}{\frac{\mathcal{B}\left|\gamma_{c}\right| \alpha_{c}}{m}(1+\sqrt{G})(1+\sqrt{\hat{G}})(\|q\| \sqrt{\hat{G}}+\|\hat{q}\| \sqrt{G})}
\end{aligned}
$$


then by lemma 5.2.5 we have the projection paraboloid metric is equivalent to the projection cone metric. Moreover by theorem 6.2.1 we get the extended paraboloid metric is equivalent to its a projection metric. The proof is complete.

Theorem 6.2.3 Any projection hyperboloid metric is equivalent to any projection cone metric. Moreover any extended hyperboloid metric is equivalent to its projection metric.

Proof We have shown before that

$$
\begin{aligned}
& \frac{\hat{t}_{h}\|\hat{q}\|-t_{h}\|q\|}{\hat{t_{c}}\|\hat{q}\|-t_{c}\|q\|} \\
& =\frac{-\alpha_{h}\left(\mathcal{C}(\|q\|-\|\hat{q}\|)(\|q\| \sqrt{\hat{E}}+\|\hat{q}\| \sqrt{E})+\left(\mathcal{C}^{2}+\mathcal{C} \alpha_{h}\right)\left(\|q\|^{2}-\|\hat{q}\|^{2}\right)\right)}{(\mathcal{C}+\sqrt{E})(\mathcal{C}+\sqrt{\hat{E}})(\|q\| \sqrt{\hat{E}}+\|\hat{q}\| \sqrt{E})}
\end{aligned}
$$

By lemma 5.2.5 we have the projection hyperboloid metric is equivalent to the projection cone metric. Moreover by theorem 6.2.1 we get the extended hyperboloid metric is equivalent to its a projection metric. The proof is complete.

\subsection{Nonequivalence Between A Projection Sphere Metric with $\alpha_{s} \neq 0$ and A Projection Cone Metric}

In this section let us observe that nonequivalence between a projection sphere metric with $\alpha_{s} \neq 0$ and a projection cone metric.

Theorem 6.3.1 Any projection sphere metric with $\alpha_{s} \neq 0$ is not equivalent to any projection cone metric.

Proof Recall that $\frac{t_{s}}{t_{c}}$ is bounded above and below by proposition 5.10.1. Now we need to show that the quantity

$$
\frac{\left(t_{s}\|q\|-\hat{t}_{s}\|\hat{q}\|\right)^{2}}{\left(t_{c}\|q\|-\hat{t}_{c}\|\hat{q}\|\right)^{2}}
$$




$$
\begin{aligned}
& \frac{\left(t_{s}\|q\|-\hat{t}_{s}\|\hat{q}\|\right)^{2}}{\left(t_{c}\|q\|-\hat{t}_{c}\|\hat{q}\|\right)^{2}}=\frac{\left(\|\hat{q}\|+\frac{\left|\gamma_{c}\right|}{m}\right)^{2}\left(\|q\|+\frac{\left|\gamma_{c}\right|}{m}\right)^{2}}{\left(\alpha_{c}\left(\sqrt{\hat{F}}+\frac{\mathcal{A}}{2}\right)\left(\sqrt{F}+\frac{\mathcal{A}}{2}\right)\right)^{2} \frac{\left|\gamma_{c}\right|^{2}}{m^{2}}}\{ \\
& \left.\frac{\left(-\alpha_{s} \frac{\mathcal{A}}{2}(\|q\| \sqrt{\hat{F}}+\|\hat{q}\| \sqrt{F})+\left(-\alpha_{s}\left(\frac{\mathcal{A}^{2}}{4}-\gamma_{s} \bar{\gamma}_{s} \alpha_{s}\right)(\|q\|+\|\hat{q}\|)\right)\right)^{2}}{(\|q\| \sqrt{\hat{F}}+\|\hat{q}\| \sqrt{F})^{2}}\right\}
\end{aligned}
$$

We now apply lemmas 5.2.3, 5.2.4 and 5.2.6, since $F=\frac{\mathcal{A}^{2}}{4}-\left(\|q\|^{2}+\gamma_{s} \bar{\gamma}_{s}\right) \alpha_{s}$ and $\hat{F}=\frac{\mathcal{A}^{2}}{4}-\left(\|\hat{q}\|^{2}+\gamma_{s} \bar{\gamma}_{s}\right) \alpha_{s}$, note that $\delta_{1}=\frac{\left|\gamma_{c}\right|}{m}>0, \delta_{2}=\frac{\mathcal{A}}{2}, \delta_{3}=\gamma_{s} \bar{\gamma}_{s}$, $\delta_{4}=-\alpha_{s}>0, \delta_{5}=-\alpha_{s}\left(\frac{\mathcal{A}^{2}}{4}-\gamma_{s} \bar{\gamma}_{s} \alpha_{s}\right)>0$ and $\delta_{6}=-\alpha_{s} \frac{\mathcal{A}}{2}<0$. Also we can note that $\delta_{2}^{2}+\delta_{3} \delta_{4}>0$ and $\delta_{6}+\frac{\delta_{5}}{\sqrt{\delta_{2}^{2}+\delta_{3} \delta_{4}}} \geq 0$. The the result follow.

Corollary 6.3.2 Any projection ellipsoid metric with $\alpha_{e} \neq 0$ is not equivalent to any projection cone metric.

\subsection{Nonequivalence Between A Projection Sphere Metric with $\alpha_{s}=0$ and A Projection El- lipsoid Metric with $\alpha_{e}=0$}

Theorem 6.4.1 Any projection sphere metric with $\alpha_{s}=0$ is not equivalent to any projection ellipsoid metric with $\alpha_{e}=0$.

Proof Recall that $\frac{t_{s_{0}}}{t_{e_{0}}}$ is bounded above and below by lemma 5.7.2. Now we need to show that the quantity

$$
\frac{\left(t_{s_{0}}\|q\|-\hat{t}_{s_{0}}\|\hat{q}\|\right)^{2}}{\left(t_{e_{0}}\|q\|-\hat{t}_{e_{0}}\|\hat{q}\|\right)^{2}}
$$

is not bounded.

$$
\begin{aligned}
& \frac{\left(t_{s_{0}}\|q\|-\hat{t}_{s_{0}}\|\hat{q}\|\right)^{2}}{\left(t_{e_{0}}\|q\|-\hat{t}_{e_{0}}\|\hat{q}\|\right)^{2}} \\
& =\frac{-\mathcal{A}_{0}(\|q\|-\|\hat{q}\|)\left(\|\hat{q}\|\|q\|-\gamma_{s_{0}} \bar{\gamma}_{s_{0}}\right)\left[\|q\|^{2}-\mathcal{D}_{0}\right]\left[\|\hat{q}\|^{2}-\mathcal{D}_{0}\right]}{-2 \mathcal{D}_{0}(\|q\|-\|\hat{q}\|)\left(\|\hat{q}\|\|q\|+\mathcal{D}_{0}\right)\left[\|q\|^{2}+\gamma_{s_{0}} \bar{\gamma}_{s_{0}}\right]\left[\|\hat{q}\|^{2}+\gamma_{s_{0}} \bar{\gamma}_{s_{0}}\right]} \\
& =\frac{-\mathcal{A}_{0}\left(\|\hat{q}\|\|q\|-\gamma_{s_{0}} \bar{\gamma}_{s_{0}}\right)\left[\|q\|^{2}-\mathcal{D}_{0}\right]\left[\|\hat{q}\|^{2}-\mathcal{D}_{0}\right]}{-2 \mathcal{D}_{0}\left(\|\hat{q}\|\|q\|+\mathcal{D}_{0}\right)\left[\|q\|^{2}+\gamma_{s_{0}} \bar{\gamma}_{s_{0}}\right]\left[\|\hat{q}\|^{2}+\gamma_{s_{0}} \bar{\gamma}_{s_{0}}\right]}
\end{aligned}
$$


Notice that when $\alpha_{s}=0$ then $\mathcal{A}_{0} \neq 0$, and when $\alpha_{e} \neq 0$ then $\mathcal{D}_{0} \neq 0$. We proceed to show that

$$
\frac{\hat{t}_{s}\|\hat{q}\|-t_{s}\|q\|}{\hat{t}_{e_{0}}\|\hat{q}\|-t_{e_{0}}\|q\|}=0
$$

for some value of $\|q\|$ and $\|\hat{q}\|$, in the set $(0, \infty) \times(0, \infty)$. Assume $q=w \hat{q}$ with $w>0$ and $w \neq 1$, therefore when $\|q\|=\sqrt{\frac{\gamma_{s_{0}} \bar{\gamma}_{s_{0}}}{w}}$ the quantity

$$
\frac{\hat{t}_{s}\|\hat{q}\|-t_{s}\|q\|}{\hat{t}_{e_{0}}\|\hat{q}\|-t_{e_{0}}\|q\|}
$$

is equal to zero. Moreover,If $\|q\| \longrightarrow \sqrt{\frac{-\mathcal{D}_{0}}{w}}$ then

$$
\left|\frac{\hat{t}_{s}\|\hat{q}\|-t_{s}\|q\|}{\hat{t}_{e_{0}}\|\hat{q}\|-t_{e_{0}}\|q\|}\right| \longrightarrow \infty
$$

The result follow.

\subsection{Nonequivalence Between A Projection Sphere Metric with $\alpha_{s}=0$ and A Projection Cone Metric}

Theorem 6.5.1 Any projection sphere metric with $\alpha_{s}=0$ is not equivalent to any projection cone metric.

Proof We compute

$$
\frac{\left(t_{s_{0}}\|q\|-\hat{t}_{s_{0}}\|\hat{q}\|\right)^{2}}{\left(t_{c}\|q\|-\hat{t}_{c}\|\hat{q}\|\right)^{2}}
$$

to get

$$
\begin{aligned}
\frac{\left(t_{s_{0}}\|q\|-\hat{t}_{s_{0}}\|\hat{q}\|\right)^{2}}{\left(t_{c}\|q\|-\hat{t}_{c}\|\hat{q}\|\right)^{2}} & =\frac{-\mathcal{A}_{0}(\|q\|-\|\hat{q}\|)\left(\|\hat{q}\|\|q\|-\gamma_{s_{0}} \bar{\gamma}_{s_{0}}\right)\left(\|\hat{q}\|+\frac{\left|\gamma_{c}\right|}{m}\right)\left(\|q\|+\frac{\left|\gamma_{c}\right|}{m}\right)}{\alpha_{c}(\|q\|-\|\hat{q}\|)\left[\|q\|^{2}+\gamma_{s_{0}} \bar{\gamma}_{s_{0}}\right]\left[\|\hat{q}\|^{2}+\gamma_{s_{0}} \bar{\gamma}_{s_{0}}\right]} \\
& =\frac{-\mathcal{A}_{0}\left(\|\hat{q}\|\|q\|-\gamma_{s_{0}} \bar{\gamma}_{s_{0}}\right)\left(\|\hat{q}\|+\frac{\left|\gamma_{c}\right|}{m}\right)\left(\|q\|+\frac{\left|\gamma_{c}\right|}{m}\right)}{\alpha_{c}\left[\|q\|^{2}+\gamma_{s_{0}} \bar{\gamma}_{s_{0}}\right]\left[\|\hat{q}\|^{2}+\gamma_{s_{0}} \bar{\gamma}_{s_{0}}\right]}
\end{aligned}
$$


Notice that

$$
\frac{\left(\hat{t}_{s_{0}}(\|\hat{q}\|)\right)^{2}}{\left(\hat{t}_{c}(\|\hat{q}\|)\right)^{2}} \longrightarrow 0
$$

as $\|\hat{q}\| \longrightarrow \infty$. We proceed to show that

$$
\frac{\left(t_{s_{0}}\|q\|-\hat{t}_{s_{0}}\|\hat{q}\|\right)^{2}}{\left(t_{c}\|q\|-\hat{t}_{c}\|\hat{q}\|\right)^{2}}=0
$$

for some value of $\|q\|$ and $\|\hat{q}\|$, in the set $(0, \infty) \times(0, \infty)$. Assume $q=w \hat{q}$ with $w>0$ and $w \neq 1$, therefore when $\|q\|=\sqrt{\frac{\gamma_{s_{0}} \bar{\gamma}_{s_{0}}}{w}}$ the quantity

$$
\frac{\left(t_{s_{0}}\|q\|-\hat{t}_{s_{0}}\|\hat{q}\|\right)^{2}}{\left(t_{c}\|q\|-\hat{t}_{c}\|\hat{q}\|\right)^{2}}
$$

is equal to zero, and the the result follow.

\subsection{Nonequivalence Between A Projection El- lipsoid Metric with $\alpha_{e}=0$ and A Projec- tion Cone Metric}

Theorem 6.6.1 Any projection ellipsoid metric with $\alpha_{e}=0$ is not equivalent to any projection cone metric.

Proof We compute

$$
\frac{\left(t_{e_{0}}\|q\|-\hat{t}_{e_{0}}\|\hat{q}\|\right)^{2}}{\left(t_{c}\|q\|-\hat{t}_{c}\|\hat{q}\|\right)^{2}}
$$

to get

$$
\begin{aligned}
\frac{\left(t_{e_{0}}\|q\|-\hat{t}_{e_{0}}\|\hat{q}\|\right)^{2}}{\left(t_{c}\|q\|-\hat{t}_{c}\|\hat{q}\|\right)^{2}} & =\frac{-2 \mathcal{D}_{0}(\|q\|-\|\hat{q}\|)\left(\|\hat{q}\|\|q\|+\mathcal{D}_{0}\right)\left(\|\hat{q}\|+\frac{\left|\gamma_{c}\right|}{m}\right)\left(\|q\|+\frac{\left|\gamma_{c}\right|}{m}\right)}{\alpha_{c}(\|q\|-\|\hat{q}\|)\left[\|q\|^{2}-\mathcal{D}_{0}\right]\left[\|\hat{q}\|^{2}-\mathcal{D}_{0}\right]} \\
& =\frac{-2 \mathcal{D}_{0}\left(\|\hat{q}\|\|q\|+\mathcal{D}_{0}\right)\left(\|\hat{q}\|+\frac{\left|\gamma_{c}\right|}{m}\right)\left(\|q\|+\frac{\left|\gamma_{c}\right|}{m}\right)}{\alpha_{c}\left[\|q\|^{2}-\mathcal{D}_{0}\right]\left[\|\hat{q}\|^{2}-\mathcal{D}_{0}\right]}
\end{aligned}
$$

Notice that

$$
\frac{\left(\hat{t}_{e_{0}}(\|\hat{q}\|)\right)^{2}}{\left(\hat{t}_{c}(\|\hat{q}\|)\right)^{2}} \longrightarrow 0
$$


as $\|\hat{q}\| \longrightarrow \infty$. We proceed to show that

$$
\frac{\left(t_{e_{0}}\|q\|-\hat{t}_{e_{0}}\|\hat{q}\|\right)^{2}}{\left(t_{c}\|q\|-\hat{t}_{c}\|\hat{q}\|\right)^{2}}=0
$$

for some value of $\|q\|$ and $\|\hat{q}\|$, in the set $(0, \infty) \times(0, \infty)$. Assume $q=w \hat{q}$ with $w>0$ and $w \neq 1$, therefore when $\|q\|=\sqrt{\frac{\mathcal{D}_{0}}{w}}$ the quantity

$$
\frac{\left(t_{e_{0}}\|q\|-\hat{t}_{e_{0}}\|\hat{q}\|\right)^{2}}{\left(t_{c}\|q\|-\hat{t}_{c}\|\hat{q}\|\right)^{2}}
$$

is equal to zero, and the the result follow.

\subsection{Nonequivalence Between The Projection Sphere Metrics with $\alpha_{s} \neq 0$ and The Pro- jection Sphere Metrics with $\alpha_{s}=0$}

In this section let us observe that nonequivalence between a projection sphere metric with $\alpha_{s} \neq 0$ and a projection sphere metric when $\alpha_{s}=0$. We have shown before that $t_{s}$ when $\alpha_{s}=0$ as following

$$
t_{s_{0}}=\frac{-\mathcal{A}_{0}}{\|q\|^{2}+\gamma_{s_{0}} \bar{\gamma}_{s_{0}}}
$$

and

$$
\hat{t}_{s_{0}}=\frac{-\mathcal{A}_{0}}{\|\hat{q}\|^{2}+\gamma_{s_{0}} \bar{\gamma}_{s_{0}}}
$$


thus

$$
\begin{aligned}
& \hat{t}_{s_{0}}\|\hat{q}\|-t_{s_{0}}\|q\|=\frac{-\mathcal{A}_{0}\|\hat{q}\|}{\|\hat{q}\|^{2}+\gamma_{s_{0}} \bar{\gamma}_{s_{0}}}-\frac{-\mathcal{A}_{0}\|q\|}{\|q\|^{2}+\gamma_{s_{0}} \bar{\gamma}_{s_{0}}} \\
& =\frac{\left[\|q\|^{2}+\gamma_{s_{0}} \bar{\gamma}_{s_{0}}\right]\left[-\mathcal{A}_{0}\right]\|\hat{q}\|-\left[\|\hat{q}\|^{2}+\gamma_{s_{0}} \bar{\gamma}_{s_{0}}\right]\left[-\mathcal{A}_{0}\right]\|q\|}{\left[\|q\|^{2}+\gamma_{s_{0}} \bar{\gamma}_{s_{0}}\right]\left[\|\hat{q}\|^{2}+\gamma_{s_{0}} \bar{\gamma}_{s_{0}}\right]} \\
& =\frac{-\mathcal{A}_{0}\|\hat{q}\|\|q\|^{2}-\mathcal{A}_{0}\|\hat{q}\| \gamma_{s_{0}} \bar{\gamma}_{s_{0}}+\mathcal{A}_{0}\|q\|\|\hat{q}\|^{2}+\mathcal{A}_{0}\|q\| \gamma_{s_{0}} \bar{\gamma}_{s_{0}}}{\left[\|q\|^{2}+\gamma_{s_{0}} \bar{\gamma}_{s_{0}}\right]\left[\|\hat{q}\|^{2}+\gamma_{s_{0}} \bar{\gamma}_{s_{0}}\right]} \\
& =\frac{-\mathcal{A}_{0}\|\hat{q}\|\|q\|(\|q\|-\|\hat{q}\|)+\mathcal{A}_{0} \gamma_{s_{0}} \bar{\gamma}_{s_{0}}(\|q\|-\|\hat{q}\|)}{\left[\|q\|^{2}+\gamma_{s_{0}} \bar{\gamma}_{s_{0}}\right]\left[\|\hat{q}\|^{2}+\gamma_{s_{0}} \bar{\gamma}_{s_{0}}\right]} \\
& =\frac{-\mathcal{A}_{0}(\|q\|-\|\hat{q}\|)\left(\|\hat{q}\|\|q\|-\gamma_{s_{0}} \bar{\gamma}_{s_{0}}\right)}{\left[\|q\|^{2}+\gamma_{s_{0}} \bar{\gamma}_{s_{0}}\right]\left[\|\hat{q}\|^{2}+\gamma_{s_{0}} \bar{\gamma}_{s_{0}}\right]} .
\end{aligned}
$$

Proposition 6.7.1 Any a projection sphere metric with $\alpha_{s} \neq 0$ is nonequivalent to any projection sphere metric with $\alpha_{s}=0$.

Proof We first determine various expressions for $\hat{t}_{s}\|\hat{q}\|-t_{s}\|q\|$ when $\alpha_{s} \neq 0$ that will be shown to be useful in the sequel. Since

$$
\begin{aligned}
t_{s}= & \frac{-\frac{\mathcal{A}}{2}+\sqrt{F}}{\|q\|^{2}+\gamma_{s} \bar{\gamma}_{s}}=\frac{-\frac{\mathcal{A}}{2}+\sqrt{F}}{\|q\|^{2}+\gamma_{s} \bar{\gamma}_{s}}\left(\frac{\sqrt{F}+\frac{\mathcal{A}}{2}}{\sqrt{F}+\frac{\mathcal{A}}{2}}\right) \\
& =\frac{F-\frac{\mathcal{A}^{2}}{4}}{\left(\|q\|^{2}+\gamma_{s} \bar{\gamma}_{s}\right)\left(\sqrt{F}+\frac{\mathcal{A}}{2}\right)}=\frac{\frac{\mathcal{A}^{2}}{4}-\left(\|q\|^{2}+\gamma_{s} \bar{\gamma}_{s}\right) \alpha_{s}-\frac{\mathcal{A}^{2}}{4}}{\left(\|q\|^{2}+\gamma_{s} \bar{\gamma}_{s}\right)\left(\sqrt{F}+\frac{\mathcal{A}}{2}\right)} \\
& =\frac{-\alpha_{s}}{\sqrt{F}+\frac{\mathcal{A}}{2}}
\end{aligned}
$$

also

$$
\hat{t}_{s}=\frac{-\alpha_{s}}{\sqrt{\hat{F}}+\frac{\mathcal{A}}{2}}
$$


thus we have

$$
\begin{aligned}
& \hat{t}_{s}\|\hat{q}\|-t_{s}\|q\|=\frac{-\alpha_{s}\|\hat{q}\|}{\sqrt{\hat{F}}+\frac{\mathcal{A}}{2}}-\frac{-\alpha_{s}\|q\|}{\sqrt{F}+\frac{\mathcal{A}}{2}} \\
& =\frac{-\alpha_{s}\|\hat{q}\|\left(\sqrt{F}+\frac{\mathcal{A}}{2}\right)-\left(-\alpha_{s}\|q\|\right)\left(\sqrt{\hat{F}}+\frac{\mathcal{A}}{2}\right)}{\left(\sqrt{\hat{F}}+\frac{\mathcal{A}}{2}\right)\left(\sqrt{F}+\frac{\mathcal{A}}{2}\right)} \\
& =\frac{\alpha_{s}(\|q\| \sqrt{\hat{F}}-\|\hat{q}\| \sqrt{F})+\alpha_{s} \frac{\mathcal{A}}{2}(\|q\|-\|\hat{q}\|)}{\left(\sqrt{\hat{F}}+\frac{\mathcal{A}}{2}\right)\left(\sqrt{F}+\frac{\mathcal{A}}{2}\right)} .
\end{aligned}
$$

We are going to elaborate on two quantities, and we use a simple trick multiply by the conjugate and factoring some terms.

$$
\begin{aligned}
& \sqrt{\hat{F}}\|q\|-\sqrt{F}\|\hat{q}\| \\
= & \frac{\left.\left(\frac{\mathcal{A}^{2}}{4}-\alpha_{s}\left[\|\hat{q}\|^{2}+\gamma_{s} \bar{\gamma}_{s}\right]\right)\|q\|^{2}-\left(\frac{\mathcal{A}^{2}}{4}-\alpha_{s}\left[\|q\|^{2}+\gamma_{s} \bar{\gamma}_{s}\right]\right)\|\hat{q}\|^{2}\right)}{(\sqrt{\hat{F}}\|q\|+\sqrt{F}\|\hat{q}\|)} \\
= & \frac{\frac{\mathcal{A}^{2}}{4}\|q\|^{2}-\alpha_{s}\|\hat{q}\|^{2}\|q\|^{2}-\alpha_{s} \gamma_{s} \bar{\gamma}_{s}\|q\|^{2}-\frac{\mathcal{A}^{2}}{4}\|\hat{q}\|^{2}+\alpha_{s}\|q\|^{2}\|\hat{q}\|^{2}+\alpha_{s} \gamma_{s} \bar{\gamma}_{s}\|\hat{q}\|^{2}}{(\sqrt{\hat{F}}\|q\|+\sqrt{F}\|\hat{q}\|)} \\
= & \frac{\frac{\mathcal{A}^{2}}{4}\|q\|^{2}-\alpha_{s} \gamma_{s} \bar{\gamma}_{s}\|q\|^{2}-\frac{\mathcal{A}^{2}}{4}\|\hat{q}\|^{2}+\alpha_{s} \gamma_{s} \bar{\gamma}_{s}\|\hat{q}\|^{2}}{(\sqrt{\hat{F}}\|q\|+\sqrt{F}\|\hat{q}\|)} \\
= & \frac{\left(\frac{\mathcal{A}^{2}}{4}-\alpha_{s} \gamma_{s} \bar{\gamma}_{s}\right)\left(\|q\|^{2}-\|\hat{q}\|^{2}\right)}{(\sqrt{\hat{F}}\|q\|+\sqrt{F}\|\hat{q}\|)} \\
= & (\|q\|-\|\hat{q}\|)(\|q\|+\|\hat{q}\|) \frac{\left(\frac{\mathcal{A}^{2}}{4}-\alpha_{s} \gamma_{s} \bar{\gamma}_{s}\right)}{(\sqrt{\hat{F}}\|q\|+\sqrt{F}\|\hat{q}\|)}
\end{aligned}
$$

The quantity

$$
\frac{\left(\frac{\mathcal{A}^{2}}{4}-\alpha_{s} \gamma_{s} \bar{\gamma}_{s}\right)}{(\sqrt{\hat{F}}\|q\|+\sqrt{F}\|\hat{q}\|)}>0,
$$

is expected to be a continuous function in both variables $\|q\|,\|\hat{q}\|$ in $(0, \infty) \times$ 
$(0, \infty)$. We are ready now to calculate the ratio

$$
\begin{aligned}
\frac{\hat{t}_{s_{0}}\|\hat{q}\|-t_{s_{0}}\|q\|}{\hat{t}_{s}\|\hat{q}\|-t_{s}\|q\|}=\frac{\frac{-\mathcal{A}_{0}(\|q\|-\|\hat{q}\|)\left(\|\hat{q}\|\|q\|-\gamma_{s_{0}} \bar{\gamma}_{s_{0}}\right)}{\left[\|q\|^{2}+\gamma_{s_{0}} \bar{\gamma}_{s_{0}}\right]\left[\|\hat{q}\|^{2}+\gamma_{s_{0}} \bar{s}_{s_{0}}\right]}}{\left(\hat{t} t_{s}\|\hat{q}\|-t_{e}\|q\|\right)} \\
=\frac{-\mathcal{A}_{0}(\|q\|-\|\hat{q}\|)\left(\|\hat{q}\|\|q\|-\gamma_{s_{0}} \bar{\gamma}_{s_{0}}\right)}{\left(\|q\|^{2}+\gamma_{s_{0}} \bar{\gamma}_{s_{0}}\right)\left(\|\hat{q}\|^{2}+\gamma_{s_{0}} \bar{\gamma}_{s_{0}}\right)\left(\hat{t}_{s}\|\hat{q}\|-t_{s}\|q\|\right)} \\
=\frac{-\mathcal{A}_{0}(\|q\|-\|\hat{q}\|)\left(\|\hat{q}\|\|q\|-\gamma_{s_{0}} \bar{\gamma}_{s_{0}}\right)\left(\sqrt{\hat{F}}+\frac{\mathcal{A}}{2}\right)\left(\sqrt{F}+\frac{\mathcal{A}}{2}\right)}{\left(\|q\|^{2}+\gamma_{s_{0}} \bar{\gamma}_{s_{0}}\right)\left(\|\hat{q}\|^{2}+\gamma_{s_{0}} \bar{\gamma}_{s_{0}}\right)\left(\alpha_{s}(\|q\| \sqrt{\hat{F}}-\|\hat{q}\| \sqrt{F})+\alpha_{s} \frac{\mathcal{A}}{2}(\|q\|-\|\hat{q}\|)\right)} \\
=\frac{-\mathcal{A}_{0}(\|q\|-\|\hat{q}\|)\left(\|\hat{q}\|\|q\|-\gamma_{s_{0}} \bar{\gamma}_{s_{0}}\right)\left(\sqrt{\hat{F}}+\frac{\mathcal{A}}{2}\right)\left(\sqrt{F}+\frac{\mathcal{A}}{2}\right)}{\alpha_{s}\left(\|q\|^{2}+\gamma_{s_{0}} \bar{\gamma}_{s_{0}}\right)\left(\|\hat{q}\|^{2}+\gamma_{s_{0}} \bar{\gamma}_{s_{0}}\right)\left((\|q\|-\|\hat{q}\|)(\|q\|+\|\hat{q}\|) \frac{\left(\frac{\mathcal{A}^{2}}{4}-\alpha_{s} \gamma_{s} \bar{\gamma}_{s}\right)}{(\sqrt{\hat{F}}\|q\|+\sqrt{F}\|\hat{q}\|)}\right)} \\
+\frac{\mathcal{A}_{0}(\|q\|-\|\hat{q}\|)\left(\|\hat{q}\|\|q\|-\gamma_{s_{0}} \bar{\gamma}_{s_{0}}\right)\left(\sqrt{\hat{F}}+\frac{\mathcal{A}}{2}\right)\left(\sqrt{F}+\frac{\mathcal{A}}{2}\right)}{\alpha_{s}\left(\|q\|^{2}+\gamma_{s_{0}} \bar{\gamma}_{s_{0}}\right)\left(\|\hat{q}\|^{2}+\gamma_{s_{0}} \bar{\gamma}_{s_{0}}\right)\left(\frac{\mathcal{A}}{2}(\|q\|-\|\hat{q}\|)\right)} \\
\quad-\mathcal{A}_{0}\left(\|\hat{q}\|\|q\|-\gamma_{s_{0}} \bar{\gamma}_{s_{0}}\right)\left(\sqrt{\hat{F}}+\frac{\mathcal{A}}{2}\right)\left(\sqrt{F}+\frac{\mathcal{A}}{2}\right) \\
=\frac{\alpha_{s}\left(\|q\|^{2}+\gamma_{s_{0}} \bar{\gamma}_{s_{0}}\right)\left(\|\hat{q}\|^{2}+\gamma_{s_{0}} \bar{\gamma}_{s_{0}}\right)\left(\frac{\mathcal{A}}{2}+(\|q\|+\|\hat{q}\|) \frac{\frac{\mathcal{A}^{2}}{4}-\alpha_{s} \gamma_{s} \bar{\gamma}_{s}}{\sqrt{\hat{F}}\|q\|+\sqrt{F}\|\hat{q}\|}\right)}{(6.7 .4)}
\end{aligned}
$$

We proceed to show that

$$
\frac{\hat{t}_{s}\|\hat{q}\|-t_{s}\|q\|}{\hat{t}_{c}\|\hat{q}\|-t_{c}\|q\|}=0
$$

for some value of $\|q\|$ and $\|\hat{q}\|$, in the set $(0, \infty) \times(0, \infty)$. Assume $q=w \hat{q}$ with $w>0$ and $w \neq 1$, therefore when $\|q\|=\sqrt{\frac{\gamma_{s_{0}} \bar{\gamma}_{s_{0}}}{w}}$ the quantity

$$
\frac{\hat{t}_{s}\|\hat{q}\|-t_{s}\|q\|}{\hat{t}_{c}\|\hat{q}\|-t_{c}\|q\|}
$$

is equal to zero, and the the result follow. 


\subsection{The Projection of Ellipsoid Metrics is Not Equivalent to The Extended Ellipsoid Met- $\operatorname{rics} \alpha_{e} \neq 0$}

Theorem 6.8.1 Any an extended ellipsoid metrics $\alpha_{e} \neq 0$ is not equivalent to its a projection metric.

Proof We proceed to show that

$$
\left|\frac{\hat{t}_{e}-t_{e}}{t_{e}\|q\|-\hat{t}_{e}\|\hat{q}\|}\right|=0
$$

for some value of $\|q\|$ and $\|\hat{q}\|$, in the set $(0, \infty) \times(0, \infty)$.

$$
\begin{aligned}
& \frac{\hat{t}_{e}-t_{e}}{t_{e}\|q\|-\hat{t}_{e}\|\hat{q}\|}=\frac{\frac{\alpha_{e}^{2}\left(\|q\|^{2}-\|\hat{q}\|^{2}\right)}{(\sqrt{\hat{\mathcal{E}}+\mathcal{D}})(\sqrt{\mathcal{E}}+\mathcal{D})(\sqrt{\mathcal{E}}+\sqrt{\hat{\mathcal{E}}})}}{\frac{-\alpha_{e} \mathcal{D}(\|q\|-\|\hat{q}\|)(\|q\| \sqrt{\hat{\mathcal{E}}}+\|\hat{q}\| \sqrt{\mathcal{E}})+\left(-\alpha_{e}\left(\mathcal{D}^{2}+\mathcal{D} \alpha_{e}\right)\left(\|q\|^{2}-\|\hat{q}\|^{2}\right)\right)}{(\sqrt{\hat{\mathcal{E}}}+\mathcal{D})(\sqrt{\mathcal{E}}+\mathcal{D})(\|q\| \sqrt{\hat{\mathcal{E}}}+\|\hat{q}\| \sqrt{\mathcal{E}})}} \\
& =\frac{\alpha_{e}^{2}(\|q\|+\|\hat{q}\|)}{\sqrt{\mathcal{E}}+\sqrt{\hat{\mathcal{E}}}}\{ \\
& \left.\frac{-\alpha_{e} \mathcal{D}(\|q\| \sqrt{\hat{\mathcal{E}}}+\|\hat{q}\| \sqrt{\mathcal{E}})+\left(-\alpha_{e}\left(\mathcal{D}^{2}+\mathcal{D} \alpha_{e}\right)(\|q\|+\|\hat{q}\|)\right)}{\|q\| \sqrt{\hat{\mathcal{E}}}+\|\hat{q}\| \sqrt{\mathcal{E}}}\right\} \\
& =\frac{\alpha_{e}^{2}(\|q\|+\|\hat{q}\|)}{\sqrt{\mathcal{E}}+\sqrt{\hat{\mathcal{E}}}}\left(\frac{1}{-\alpha_{e} \mathcal{D}+\frac{-\alpha_{e}\left(\mathcal{D}^{2}+\mathcal{D} \alpha_{e}\right)(\|q\|+\|\hat{q}\|)}{\|q\| \sqrt{\hat{\mathcal{E}}}+\|\hat{q}\| \sqrt{\mathcal{E}}}}\right)
\end{aligned}
$$

Consider

$$
J=\frac{1}{-\alpha_{e} \mathcal{D}+\frac{-\alpha_{e}\left(\mathcal{D}^{2}+\mathcal{D} \alpha_{e}\right)(\|q\|+\|\hat{q}\|)}{\|q\| \sqrt{\hat{\mathcal{E}}}+\|\hat{q}\| \sqrt{\mathcal{E}}}}
$$

Assume $\hat{q}=w q$ with $w>0$ and $w \neq 1$, we obtain

$$
\begin{aligned}
J & =\frac{1}{-\alpha_{e} \mathcal{D}+\frac{-\alpha_{e}\left(\mathcal{D}^{2}+\mathcal{D} \alpha_{e}\right)\|q\|(1+w)}{\|q\| \sqrt{\mathcal{D}^{2}+\mathcal{D} \alpha_{e}-\alpha_{e} w\|q\|^{2}}+w\|q\| \sqrt{\mathcal{D}^{2}+\mathcal{D} \alpha_{e}-\alpha_{e}\|q\|^{2}}}} \\
& =\frac{1}{-\alpha_{e} \mathcal{D}+\frac{-\alpha_{e}\left(\mathcal{D}^{2}+\mathcal{D} \alpha_{e}\right)(1+w)}{\sqrt{\mathcal{D}^{2}+\mathcal{D} \alpha_{e}-\alpha_{e} w\|q\|^{2}}+w \sqrt{\mathcal{D}^{2}+\mathcal{D} \alpha_{e}-\alpha_{e}\|q\|^{2}}}}
\end{aligned}
$$


Compute the limit of $J$ as $\|q\| \longrightarrow \infty$ and as $\|\hat{q}\| \longrightarrow \infty$ we get

$$
\lim _{\substack{\|q\| \rightarrow \infty \\\|\hat{q}\| \rightarrow \infty}} J=\frac{1}{-\alpha_{e} \mathcal{D}}<0
$$

Compute the limit of $J$ as $\|q\| \longrightarrow 0$ and as $\|\hat{q}\| \longrightarrow 0$ we get

$$
\begin{aligned}
\lim _{\substack{\|q\| \rightarrow 0 \\
\|\hat{q}\| \rightarrow 0}} J & =\lim _{\substack{\|q\| \longrightarrow 0 \\
\|\hat{q}\| \longrightarrow 0}} \frac{1}{-\alpha_{e} \mathcal{D}+\frac{-\alpha_{e}\left(\mathcal{D}^{2}+\mathcal{D} \alpha_{e}\right)(1+w)}{\sqrt{\mathcal{D}^{2}+\mathcal{D} \alpha_{e}-\alpha_{e} w\|q\|^{2}}+w \sqrt{\mathcal{D}^{2}+\mathcal{D} \alpha_{e}-\alpha_{e}\|q\|^{2}}}} \\
& =\frac{1}{-\alpha_{e} \mathcal{D}+\frac{-\alpha_{e}\left(\mathcal{D}^{2}+\mathcal{D} \alpha_{e}\right)(1+w)}{\sqrt{\mathcal{D}^{2}+\mathcal{D} \alpha_{e}+w \sqrt{\mathcal{D}^{2}+\mathcal{D} \alpha_{e}}}}}=\frac{-1}{-\alpha_{e} \mathcal{D}+\frac{-\alpha_{e}\left(\mathcal{D}^{2}+\mathcal{D} \alpha_{e}\right)}{\sqrt{\mathcal{D}^{2}+\mathcal{D} \alpha_{e}}}} \\
& =\frac{-1}{\alpha_{e} \mathcal{D}\left(1+\frac{-\alpha_{e}\left(\mathcal{D}+\alpha_{e}\right)}{\sqrt{\mathcal{D}^{2}+\mathcal{D} \alpha_{e}}}\right)}=\frac{-1}{\alpha_{e} \mathcal{D}\left(1+\frac{-\left(-\mathcal{D}-\alpha_{e}\right)}{\left.\sqrt{-\mathcal{D} \sqrt{-\mathcal{D}-\alpha_{e}}}\right)}\right.} \\
& =\frac{-1}{\alpha_{e} \mathcal{D}\left(1+\frac{-\alpha_{e}\left(\mathcal{D}+\alpha_{e}\right)}{\sqrt{\mathcal{D}^{2}+\mathcal{D} \alpha_{e}}}\right)}=\frac{-1}{\alpha_{e} \mathcal{D}\left(1-\frac{\sqrt{-\mathcal{D}-\alpha_{e}}}{\sqrt{-\mathcal{D}}}\right)} \\
& =\frac{-1}{\alpha_{e} \mathcal{D}\left(1-\sqrt{1+\frac{\alpha_{e}}{\mathcal{D}}}\right)}>0
\end{aligned}
$$

Therefore, bu (6.8.1) and (6.8.2) the quantity

$$
\left|\frac{\hat{t}_{g}-t_{g}}{t_{g}\|q\|-\hat{t}_{g}\|\hat{q}\|}\right|
$$

is equal to zero for some value of $\|q\|$ and $\|\hat{q}\|$, in the set $(0, \infty) \times(0, \infty)$, and the the result follow.

\subsection{The Projection Sphere Metric with $\alpha_{s}=0$ is Not Equivalent to Its Extended Metric}

Lemma 6.9.1 Any projection sphere metric with $\alpha_{s}=0$ is not equivalent to its extended metric.

Proof Sine

$$
\hat{t}_{s_{0}}-t_{s_{0}}=\frac{-\mathcal{A}_{0}\left(\|q\|^{2}-\|\hat{q}\|^{2}\right)}{\left[\|q\|^{2}+\gamma_{s_{0}} \bar{\gamma}_{s_{0}}\right]\left[\|\hat{q}\|^{2}+\gamma_{s_{0}} \bar{\gamma}_{s_{0}}\right]} .
$$




$$
\begin{gathered}
\hat{t}_{s_{0}}\|\hat{q}\|-t_{s_{0}}\|q\|=\frac{-\mathcal{A}_{0}(\|q\|-\|\hat{q}\|)\left(\|\hat{q}\|\|q\|-\gamma_{s_{0}} \bar{\gamma}_{s_{0}}\right)}{\left[\|q\|^{2}+\gamma_{s_{0}} \bar{\gamma}_{s_{0}}\right]\left[\|\hat{q}\|^{2}+\gamma_{s_{0}} \bar{\gamma}_{s_{0}}\right]} . \\
\frac{\hat{t}_{s_{0}}-t_{s_{0}}}{\hat{t}_{s_{0}}\|\hat{q}\|-t_{s_{0}}\|q\|}=\frac{\frac{-\mathcal{A}_{0}\left(\|q\|^{2}-\|\hat{q}\|^{2}\right)}{\frac{\left[\|q\|^{2}+\gamma_{s_{0}} \bar{\gamma}_{s_{0}}\right]\left[\|\hat{q}\|^{2}+\gamma_{s_{0}} \bar{\gamma}_{s_{0}}\right]}{-\mathcal{A}_{0}(\|q\|-\|\hat{q}\|)\left(\|\hat{q}\|\|q\|-\gamma_{s_{0}} \bar{\gamma}_{s_{0}}\right)}}}{\left[\|q\|^{2}+\gamma_{s_{0}} \bar{\gamma}_{s_{0}}\right]\left[\|\hat{q}\|^{2}+\gamma_{s_{0}} \bar{\gamma}_{s_{0}}\right]} \\
=\frac{\|q\|^{2}-\|\hat{q}\|^{2}}{(\|q\|-\|\hat{q}\|)\left(\|\hat{q}\|\|q\|-\gamma_{s_{0}} \bar{\gamma}_{s_{0}}\right)}=\frac{\|q\|+\|\hat{q}\|}{\|\hat{q}\|\|q\|-\gamma_{s_{0}} \bar{\gamma}_{s_{0}}}
\end{gathered}
$$

Choose $q=w \hat{q}$ with $w>0$ and $w \neq 1$, then

$$
2\|q\|\|\hat{q}\|-\langle\hat{q}, q\rangle-\langle q, \hat{q}\rangle=0
$$

Therefore we are left with the quotient

$$
\frac{\|q\|+\|\hat{q}\|}{\|\hat{q}\|\|q\|+\gamma_{s_{0}} \bar{\gamma}_{s_{0}}}=\frac{w\|\hat{q}\|+\|\hat{q}\|}{w\|\hat{q}\|^{2}+\gamma_{s_{0}} \bar{\gamma}_{s_{0}}}=\frac{(w+1)\|\hat{q}\|}{w\|\hat{q}\|^{2}+\gamma_{s_{0}} \bar{\gamma}_{s_{0}}}
$$

Let $\|q\| \longrightarrow \sqrt{\frac{\gamma_{s_{0}} \bar{\gamma}_{s_{0}}}{w}}$ then we have

$$
\left|\frac{\hat{t}_{g}-t_{g}}{t_{g}\|q\|-\hat{t}_{g}\|\hat{q}\|}\right| \longrightarrow \infty
$$

By theorem 6.1.2 the result follow

\subsection{The projection Ellipsoid Metric with $\alpha_{e}=$ 0 is Not Equivalent to Its Extended Met- ric}

Lemma 6.10.1 Any projection ellipsoid metric with $\alpha_{e}=0$ is not equivalent to its extended metric.

Proof

$$
\begin{gathered}
\hat{t}_{e_{0}}-t_{e_{0}}=\frac{-2 \mathcal{D}_{0}\left(\|q\|^{2}-\|\hat{q}\|^{2}\right)}{\left[\|q\|^{2}-\mathcal{D}_{0}\right]\left[\|\hat{q}\|^{2}-\mathcal{D}_{0}\right]} \\
\hat{t}_{e_{0}}\|\hat{q}\|-t_{e_{0}}\|q\|=\frac{-2 \mathcal{D}_{0}(\|q\|-\|\hat{q}\|)\left(\|\hat{q}\|\|q\|+\mathcal{D}_{0}\right)}{\left[\|q\|^{2}-\mathcal{D}_{0}\right]\left[\|\hat{q}\|^{2}-\mathcal{D}_{0}\right]}
\end{gathered}
$$




$$
\begin{aligned}
\frac{\hat{t}_{e_{0}}-t_{e_{0}}}{\hat{t}_{e_{0}}\|\hat{q}\|-t_{e_{0}}\|q\|} & =\frac{\frac{-2 \mathcal{D}_{0}\left(\|q\|^{2}-\|\hat{q}\|^{2}\right)}{\left[\|q\|^{2}-\mathcal{D}_{0}\right]\left[\|\hat{q}\|^{2}-\mathcal{D}_{0}\right]}}{\frac{-2 \mathcal{D}_{0}(\|q\|-\|\hat{q}\|)\left(\|\hat{q}\|\|q\|+\mathcal{D}_{0}\right)}{\left[\|q\|^{2}-\mathcal{D}_{0}\right]\left[\|\hat{q}\|^{2}-\mathcal{D}_{0}\right]}} \\
& =\frac{\|q\|^{2}-\|\hat{q}\|^{2}}{(\|q\|-\|\hat{q}\|)\left(\|\hat{q}\|\|q\|+\mathcal{D}_{0}\right)}=\frac{\|q\|+\|\hat{q}\|}{\|\hat{q}\|\|q\|+\mathcal{D}_{0}}
\end{aligned}
$$

Choose $q=w \hat{q}$ with $w>0$ and $w \neq 1$, then

$$
2\|q\|\|\hat{q}\|-\langle\hat{q}, q\rangle-\langle q, \hat{q}\rangle=0 .
$$

Therefore we are left with the quotient

$$
\frac{\|q\|+\|\hat{q}\|}{\|\hat{q}\|\|q\|+\mathcal{D}_{0}}=\frac{w\|\hat{q}\|+\|\hat{q}\|}{w\|\hat{q}\|^{2}+\mathcal{D}_{0}}=\frac{(w+1)\|\hat{q}\|}{w\|\hat{q}\|^{2}+\mathcal{D}_{0}}
$$

Let $\|q\| \longrightarrow \sqrt{\frac{-\mathcal{D}_{0}}{w}}$ then we have

$$
\left|\frac{\hat{t}_{e_{0}}-t_{e_{0}}}{t_{e_{0}}\|q\|-\hat{t}_{e_{0}}\|\hat{q}\|}\right| \longrightarrow \infty
$$

By theorem 6.1.2 the result follow 


\section{Chapter 7}

\section{Conformal Mapping and Triangles Similarity in Sphere Boundization}

This chapter raises additional questions related to Ptolemy's work about conformity under nonlinear projections. Does it hold true that if the measure of all angles under a nonlinear projection of $\mathbb{H}$ on a general surface in $\mathbb{R}^{n}$ is preserved, must then the surface be a sphere? Does it hold true that if $P Q \hat{Q}$ and $P \hat{Z} Z$ generated by a nonlinear projection on a general surface in $\mathbb{R}^{n}$ preserves their similarity then the general surface must be a sphere? Can the latter two questions be answered in $\mathbb{C}^{n}$ or in a Complex Hilbert space in the affirmative? Answers to these questions will also constitute a generalization and extension in several new directions to the work of E. Kasner and J. De Cicco [34]. This work indicates that the answers to these questions should be yes. Our main result in this chapter is concerned with the construction of a multi parameter family of nonlinear "spherical" projections of a complex Hilbert space $\mathbb{H}$ on bounded "bowl" shaped subsets of spheres in an extended complex Hilbert space $\mathbb{H}_{N}$. It focuses on the properties of the images of angles under these nonlinear projections. We consider in a complex Hilbert space a family of nonlinear projections that depend on two complex valued parameters and on one real parameter. Two complex parameters $\theta_{s}, \gamma_{s}$ help define the center of the sphere and the fixed "projection point" from which projection takes place. The real parameter $R \geq 0$ denotes the radius of a sphere. Under the "spherical" projections, each point $Q$ in a complex Hilbert space $\mathbb{H}$ is matched to a point $Z$ in an "extended" complex Hilbert 
space $\mathbb{H}_{N}$ on a sphere.

Definition 7.0.1 Let $\mathcal{X}$ be an arbitrary angle in $\mathbb{H}$ and let $\mathcal{Y}$ be its image under the projection on the sphere. We say that the projection mapping is conformal if $\forall \mathcal{X}, \mathcal{Y}=\mathcal{X}$.

In order to formulate the main result of this chapter we first need to recall a result of Y. I. Gingold and H. Gingold [26] for $\mathbb{R}^{2}$ that provides the converse to a well known theorem in complex analysis. It states the following. Let $Q$ and $\hat{Q}$ be any two distinct points in $\mathbb{R}^{2}$ and let $Z$ and $\hat{Z}$ be the images of $Q$ and $\hat{Q}$ respectively under a spherical projection with fixed projection point $P$. Then, the triangles $P Q \hat{Q}$ and $P \hat{Z} Z$ are similar iff the spherical projection is the stereographic projection. In the sequel we allow $Q$ and $\hat{Q}$ to be any two distinct points in a complex Hilbert space and we let $Z$ and $\hat{Z}$ be the images of $Q$ and $\hat{Q}$, respectively, under a "spherical projection" with fixed projection point $P$. The main result of this chapter is formulated below.

Theorem 7.0.2 The following conditions are equivalent in a complex Hilbert space. i) A spherical projection is the stereographic projection. ii) The spherical projection is conformal. iii) Any two triangles $P Q \hat{Q}$ and $P \hat{Z} Z$ are similar under the spherical projection.

\subsection{The Angle Measure Between The Images of Two Curves}

Our next task is to determine the measure of the image of an angle $\mathcal{X}$ under a spherical projection $\operatorname{SPM}\left(\theta_{s}, R, \gamma_{s}\right)$. Let $\mathcal{V}$ and $\hat{\mathcal{V}}$ be two unit vectors in $\mathbb{H}$ such that $\|\mathcal{V}\|=\|\hat{\mathcal{V}}\|=1$. Then $(\overrightarrow{0}, \mathcal{V})$ and $(\overrightarrow{0,}, \mathcal{V})$ are unit vectors in $\mathbb{S}_{N}$ according to the definition $\left\langle Z_{1}, Z_{2}\right\rangle_{H_{N}}=\left\langle z_{1}, z_{2}\right\rangle+c_{1} \bar{c}_{2}$ in (2.2.2). Let $\mathcal{X}$ be the angle between $\mathcal{V}$ and $\hat{\mathcal{V}}$ in $\mathbb{H}$ as defined in (1.1.2). Then it is easily verified that

$$
\cos (\mathcal{X})=\frac{\langle\mathcal{V}, \hat{\mathcal{V}}\rangle+\langle\hat{\mathcal{V}}, \mathcal{V}\rangle}{2}=\frac{\langle(\overrightarrow{0}, \mathcal{V}),(\overrightarrow{0} \cdot \hat{\mathcal{V}})+\langle(\overrightarrow{0}, \hat{\mathcal{V}}),(\overrightarrow{0}, \mathcal{V})\rangle}{2}
$$

Namely, the measure of the angle between $\mathcal{V}$ and $\hat{\mathcal{V}}$ in $\mathbb{H}$ is the same as the measure of the angle between $(\overrightarrow{0}, \mathcal{V})$ and $(\overrightarrow{0,} \mathcal{V})$ in $S_{N}$. Let $\mathcal{W}(q)$ and $\hat{\mathcal{W}}(q)$ be two curves in $\mathbb{H}$ and let $\mathcal{H}(\delta)=q_{0}+\delta \mathcal{V}$ and $\hat{\mathcal{H}}(\delta)=q_{0}+\delta \hat{\mathcal{V}}$ be 
their tangent lines at $q_{0}$ respectively, such that $\mathcal{H}(0)=\hat{\mathcal{H}}(0)=q_{0}$ where $0 \leq \delta<\infty$. The three vectors $q_{0}, \mathcal{V}$ and $\hat{\mathcal{V}}$ create a configuration where the arrows representing $q_{0}, \mathcal{V}$ and $\hat{\mathcal{V}}$ respectively emanate from the head of the arrow representing the vector $q_{0}$. In this configuration we have three angles . The angle $\mathcal{X}$ between arrows representing $\mathcal{V}$ and $\hat{\mathcal{V}}$, the angle $\eta$ between arrows representing $q_{0}$ and $\mathcal{V}$ and the angle $\hat{\eta}$ between arrows representing $q_{0}$ and $\mathcal{V}$. We put for $\left\|q_{0}\right\| \neq 0$

$$
\begin{aligned}
\cos (\eta) & :=\frac{\left\langle\left\|q_{0}\right\|^{-1} q_{0}, \mathcal{V}\right\rangle+\left\langle\mathcal{V},\left\|q_{0}\right\|^{-1} q_{0}\right\rangle}{2}, \\
\cos (\hat{\eta}): & =\frac{\left\langle\left\|q_{0}\right\|^{-1} q_{0}, \hat{\mathcal{V}}\right\rangle+\left\langle\hat{\mathcal{V}},\left\|q_{0}\right\|^{-1} q_{0}\right\rangle}{2},\left\|q_{0}\right\| \neq 0 .
\end{aligned}
$$

Evidently, the angle between a zero vector $q_{0}$ and another vector is not well defined. However, with $U$ a unit vector in $\mathbb{H}$ and with $q_{0}=\rho U, \rho>0$ the radial limits

$$
\lim _{q_{0} \rightarrow 0 \hat{0}}\left\|q_{0}\right\|^{-1} q_{0}=\lim _{\rho \rightarrow 0^{+}} U=U
$$

do exist.

$\|\mathcal{H}(\delta)\|$ is actually a function of $q_{0} \in \mathbb{H}$ and $\delta, \delta \geq 0$. We need information about the existence and continuity of the derivative $\frac{\partial\|\mathcal{H}(\delta)\|}{\partial \delta}$ as a function of $q_{0} \in \mathbb{H}$ and $\delta, \delta \geq 0$. The derivative $\frac{\partial\|\mathcal{H}(\delta)\|}{\partial \delta}$ evaluated at $\delta=0$ is denoted by $\left.\frac{\partial\|\mathcal{H}(\delta)\|}{\partial \delta}\right|_{\delta=0}$. We also need information about the limit of the quotient $\frac{\|\mathcal{H}(\delta)\|-\|\mathcal{H}(0)\|}{\delta}$. This is given in the next Lemma.

Lemma 7.1.1 i) The derivative $\frac{\partial\|\mathcal{H}(\delta)\|}{\partial \delta}$ exists for each fixed $q_{0} \in \mathbb{H}$ and it is a continuous function of $q_{0}$ and $\delta$ for every fixed $q_{0} \neq \overrightarrow{0}, \delta \geq 0$ and $\delta$ small enough and

$$
\lim _{\delta \rightarrow 0^{+}} \frac{\|\mathcal{H}(\delta)\|-\|\mathcal{H}(0)\|}{\delta}=\lim _{\delta \rightarrow 0^{+}} \frac{\partial\|\mathcal{H}(\delta)\|}{\partial \delta}=\frac{\left\langle q_{0}, \mathcal{V}\right\rangle+\left\langle\mathcal{V}, q_{0}\right\rangle}{2 \sqrt{\left\langle q_{0}, q_{0}\right\rangle}}=\cos (\eta)
$$

ii) For $q_{0}=\overrightarrow{0}$ we have

$$
\lim _{\delta \rightarrow 0^{+}} \frac{\|\mathcal{H}(\delta)\|-\|\mathcal{H}(0)\|}{\delta}=1=\frac{\partial\|\mathcal{H}(\delta)\|}{\partial \delta} .
$$

Proof By definition we have for $q_{0} \neq \overrightarrow{0}$ 


$$
\begin{gathered}
\lim _{\delta \rightarrow 0^{+}} \frac{\|\mathcal{H}(\delta)\|-\|\mathcal{H}(0)\|}{\delta}=\lim _{\delta \rightarrow 0^{+}} \frac{\sqrt{\left\langle q_{0}+\delta V, q_{0}+\delta \mathcal{V}\right\rangle}-\sqrt{\left\langle q_{0}, q_{0}\right\rangle}}{\delta}= \\
\lim _{\delta \rightarrow 0^{+}} \frac{\left\langle q_{0}+\delta \mathcal{V}, q_{0}+\delta \mathcal{V}\right\rangle-\left\langle q_{0}, q_{0}\right\rangle}{\delta\left[\sqrt{\left\langle q_{0}+\delta \mathcal{V}, q_{0}+\delta \mathcal{V}\right\rangle}+\sqrt{\left\langle q_{0}, q_{0}\right\rangle}\right]} \\
=\lim _{\delta \rightarrow 0^{+}} \frac{\left\langle q_{0}+\delta \mathcal{V}, q_{0}+\delta \mathcal{V}\right\rangle-\left\langle q_{0}, q_{0}\right\rangle}{\delta\left[\sqrt{\left\langle q_{0}+\delta \mathcal{V}, q_{0}+\delta \mathcal{V}\right\rangle}+\sqrt{\left\langle q_{0}, q_{0}\right\rangle}\right]} \\
=\lim _{\delta \rightarrow 0^{+}} \frac{\left\langle q_{0}, \delta \mathcal{V}\right\rangle+\left\langle\delta \mathcal{V}, q_{0}\right\rangle+\langle\delta \mathcal{V}, \delta \mathcal{V}\rangle}{\delta\left[\sqrt{\left\langle q_{0}+\delta \mathcal{V}, q_{0}+\delta \mathcal{V}\right\rangle}+\sqrt{\left\langle q_{0}, q_{0}\right\rangle}\right]}=\frac{\left\langle q_{0}, \mathcal{V}\right\rangle+\left\langle\mathcal{V}, q_{0}\right\rangle}{2 \sqrt{\left\langle q_{0}, q_{0}\right\rangle}}=\cos (\eta)
\end{gathered}
$$

On the other hand for $\delta$ positive and $\delta$ small enough we have

$$
\begin{gathered}
\frac{\partial\|\mathcal{H}(\delta)\|}{\partial \delta}=\frac{\partial \sqrt{\left\langle q_{0}+\delta \mathcal{V}, q_{0}+\delta \mathcal{V}\right\rangle}}{\partial \delta}= \\
=\frac{1}{2 \sqrt{\left\langle q_{0}+\delta \mathcal{V}, q_{0}+\delta \mathcal{V}\right\rangle}} \frac{\partial\left\langle q_{0}+\delta \mathcal{V}, q_{0}+\delta \mathcal{V}\right\rangle}{\partial \delta}= \\
\frac{\left\langle q_{0}+\delta \mathcal{V}, \mathcal{V}\right\rangle+\left\langle\mathcal{V}, q_{0}+\delta \mathcal{V}\right\rangle}{2\left\|q_{0}+\delta \mathcal{V}\right\|}=\frac{\left\langle q_{0}, \mathcal{V}\right\rangle+\langle\delta \mathcal{V}, \mathcal{V}\rangle+\left\langle\mathcal{V}, q_{0}\right\rangle+\langle\mathcal{V}, \delta \mathcal{V}\rangle}{2\left\|q_{0}+\delta \mathcal{V}\right\|}= \\
=\frac{2 \delta+\left\langle q_{0}, \mathcal{V}\right\rangle+\left\langle\mathcal{V}, q_{0}\right\rangle}{2\left\|q_{0}+\delta \mathcal{V}\right\|} .
\end{gathered}
$$

Notice that the denominator $\left\langle q_{0}+\delta \mathcal{V}, q_{0}+\delta \mathcal{V}\right\rangle^{\frac{1}{2}} \neq 0$ for $0<\left\|q_{0}\right\|<\infty$ and $q_{0}$ fixed and for $\delta$ positive and $\delta$ small enough. This is so because then

$$
\begin{aligned}
\left\langle q_{0}+\delta \mathcal{V}, q_{0}+\delta \mathcal{V}\right\rangle & =\left|\left\|q_{0}\right\|^{2}+\left\langle q_{0}, \delta \mathcal{V}\right\rangle+\left\langle\delta \mathcal{V}, q_{0}\right\rangle+\langle\delta \mathcal{V}, \delta \mathcal{V}\rangle\right| \\
& \geq\left\|q_{0}\right\|^{2}-\delta\left[2\left|\left\langle q_{0}, \delta \mathcal{V}\right\rangle\right|+\delta\right]>0
\end{aligned}
$$

Thus, Evidently, $\frac{\partial\|\mathcal{H}(\delta)\|}{\partial \delta}$ is a continuous function of $q_{0} \neq \overrightarrow{0}$ and the conclusion for $q_{0} \neq \overrightarrow{0}$ follows from the comparison of (7.1.6) with (7.1.5).

In case that $q_{0}=\hat{0}$ the derivative $\frac{\partial\|\mathcal{H}(\delta)\|}{\partial \delta}=\frac{\partial \sqrt{\langle\delta \mathcal{V}, \delta \mathcal{V}\rangle}}{\partial \delta}=\frac{\partial \delta}{\partial \delta} \equiv 1$ exists . It is also consistent with the definition of the quotient $\lim _{\delta \rightarrow 0^{+}} \frac{\sqrt{\langle\delta \mathcal{V}, \delta \mathcal{V}\rangle}-0}{\delta}=1$ and

(7.1.4) follows. However, it is easily seen that the value 1 is not a continuous limit of $\frac{\left\langle q_{0}, \mathcal{V}\right\rangle+\left\langle\mathcal{V}, q_{0}\right\rangle}{2 \sqrt{\left\langle q_{0}, q_{0}\right\rangle}}$ as $q_{0} \rightarrow \overrightarrow{0}$. 
We proceed to determine $\mathcal{Y}$, the image of the angle $\mathcal{X}$ on the sphere under the spherical projection $\operatorname{SPM}\left(\theta_{s}, R, \gamma_{s}\right)$. It is given in the next Theorem.

\section{Theorem 7.1.2 Let}

$$
Z(\delta)=\left(\left(1-t_{s}(\delta)\right) \gamma_{s} u, t_{s}(\delta) \mathcal{H}(\delta)\right), \hat{Z}(\delta)=\left(\left(1-\hat{t}_{s}(\delta)\right) \gamma_{s} u, \hat{t}_{s}(\delta) \hat{\mathcal{H}}(\delta)\right)
$$

be the images of $(0, \mathcal{H}(\delta))$ and $(0, \hat{\mathcal{H}}(\delta))$ under $\operatorname{SPM}\left(\theta_{s}, R, \gamma_{s}\right)$ respectively.

Then, the angle $\mathcal{Y}$ between the curves $Z(\delta)$ and $\hat{Z}(\delta)$ that meet at $Z(0)=$ $\hat{Z}(0)$ on the sphere $S P\left(\theta_{s}, R\right)=\left\{Z \in H_{N} \mid\|Z-O\|_{\mathbb{H}_{N}}=R\right\}$ is given by

$$
\cos (\mathcal{Y})=\frac{\cos (\mathcal{X})+\mathcal{J}}{\sqrt{1+\mathcal{J}^{2}+\Lambda}}
$$

$\operatorname{Cos}(\mathcal{X})$ is the cosine of the angle between $\mathcal{H}(0)$ and $\hat{\mathcal{H}}(0), \mathcal{J}$ is given by

$$
\mathcal{J}:=\frac{\alpha}{4 \mathcal{F}(0)}\left[\left\langle q_{0}, \mathcal{V}\right\rangle+\left\langle\mathcal{V}, q_{0}\right\rangle\right]\left[\left\langle q_{0}, \hat{\mathcal{V}}\right\rangle+\left\langle\hat{\mathcal{V}}, q_{0}\right\rangle\right]=\frac{\alpha\left\|q_{0}\right\|^{2}}{\mathcal{F}(0)} \cos (\eta) \cos (\hat{\eta})
$$

and $\Lambda$ is given by

$$
\begin{aligned}
\Lambda & :=\frac{\alpha}{4 \mathcal{F}(0)}\left(\left[\left\langle q_{0}, \hat{\mathcal{V}}\right\rangle+\left\langle\hat{\mathcal{V}}, q_{0}\right\rangle\right]^{2}+\left[\left\langle q_{0}, \mathcal{V}\right\rangle+\left\langle\mathcal{V}, q_{0}\right\rangle\right]^{2}\right) \\
& =\frac{\alpha\left\|q_{0}\right\|^{2}}{\mathcal{F}(0)}\left[\cos ^{2}(\eta)+\cos ^{2}(\hat{\eta})\right] .
\end{aligned}
$$

Proof We first elaborate on the notation needed for the images (7.1.8) of the rays $\left(0, q_{0}+\delta \mathcal{V}\right)$ and $\left(0, q_{0}+\delta \hat{\mathcal{V}}\right)$ in $\mathbb{S}_{N}$ on the sphere $S P\left(\theta_{s}, R\right):=\{Z \in$ $\left.\mathbb{H}_{N}:\|Z-O\|_{H_{N}}=R\right\}$ according to section 2. Analogous to (3.5.17) we have

$$
t_{s}(\delta)=\frac{\frac{-\mathcal{A}}{2}+\sqrt{\mathcal{F}(\delta)}}{\|\mathcal{H}(\delta)\|^{2}+\gamma_{s} \bar{\gamma}_{s}}, \quad \hat{t}_{s}(\delta)=\frac{\frac{-\mathcal{A}}{2}+\sqrt{\hat{\mathcal{F}}(\delta)}}{\|\hat{\mathcal{H}}(\delta)\|^{2}+\gamma_{s} \bar{\gamma}_{s}}
$$

where

$$
\mathcal{F}(\delta)=\frac{\mathcal{A}^{2}}{4}-\alpha\left(\|\mathcal{H}(\delta)\|^{2}+\gamma_{s} \bar{\gamma}_{s}\right), \hat{\mathcal{F}}(\delta)=\frac{\mathcal{A}^{2}}{4}-\alpha\left(\|\hat{\mathcal{H}}(\delta)\|^{2}+\gamma_{s} \bar{\gamma}_{s}\right) .
$$


For $\delta=0$ we have

$$
\mathcal{F}(0)=\frac{\mathcal{A}^{2}}{4}-\alpha\left(\left\|q_{0}\right\|^{2}+\gamma_{s} \bar{\gamma}_{s}\right)=\hat{\mathcal{F}}(0) \Rightarrow t_{s}(0)=\frac{\frac{-\mathcal{A}}{2}+\sqrt{\mathcal{F}(0)}}{\left\|q_{0}\right\|^{2}+\gamma_{s} \bar{\gamma}_{s}}=\hat{t}_{s}(0)
$$

Next we evaluate the inner products of the tangent vectors to $Z(\delta)$ and $\hat{Z}(\delta)$ expressed by

$$
\left\langle\frac{\partial Z(\delta)}{\partial \delta}, \frac{\partial \hat{Z}(\delta)}{\partial \delta}\right\rangle_{H_{N}} \text { and }\left\langle\frac{\partial \hat{Z}(\delta)}{\partial \delta}, \frac{\partial Z(\delta)}{\partial \delta}\right\rangle_{H_{N}}
$$

To find the derivative of $Z(\delta)$ and $\hat{Z}(\delta)$, we must first find the derivatives of $t_{s}(\delta)$ and $\hat{t}_{s}(\delta)$

$$
\begin{aligned}
\frac{\partial t_{s}(\delta)}{\partial \delta} & =\frac{\left(\|\mathcal{H}(\delta)\|^{2}+\gamma_{s} \bar{\gamma}_{s}\right) \frac{-\alpha\|\mathcal{H}(\delta)\| \frac{\partial\|\mathcal{H}(\delta)\|}{\partial \delta}}{\sqrt{\mathcal{F}(\delta)}}}{\left(\|\mathcal{H}(\delta)\|^{2}+\gamma_{s} \bar{\gamma}_{s}\right)^{2}} \\
- & \frac{\left(\frac{-\mathcal{A}}{2}+\sqrt{\mathcal{F}(\delta)}\right)\left(2\|\mathcal{H}(\delta)\| \frac{\partial\|\mathcal{H}(\delta)\|}{\partial \delta}\right)}{\left(\|\mathcal{H}(\delta)\|^{2}+\gamma_{s} \bar{\gamma}_{s}\right)^{2}} \\
\frac{\partial \hat{t}_{s}(\delta)}{\partial \delta}= & \frac{\left(\|\hat{\mathcal{H}}(\delta)\|^{2}+\gamma_{s} \bar{\gamma}_{s}\right) \frac{-\alpha\|\hat{\mathcal{H}}(\delta)\| \frac{\partial\|\hat{\mathcal{H}}(\delta)\|}{\partial \delta}}{\sqrt{\hat{\mathcal{F}}(\delta)}}}{\left(\|\hat{\mathcal{H}}(\delta)\|^{2}+\gamma_{s} \bar{\gamma}_{s}\right)^{2}} \\
- & \frac{\left(\frac{-\mathcal{A}}{2}+\sqrt{\hat{\mathcal{F}}(\delta)}\right)\left(2\|\hat{\mathcal{H}}(\delta)\| \frac{\partial\|\hat{\mathcal{H}}(\delta)\|}{\partial \delta}\right)}{\left(\|\hat{\mathcal{H}}(\delta)\|^{2}+\gamma_{s} \bar{\gamma}_{s}\right)^{2}}
\end{aligned}
$$

where $\frac{\partial\|\mathcal{H}(\delta)\|}{\partial \delta}$ and $\frac{\partial\|\hat{\mathcal{H}}(\delta)\|}{\partial \delta}$ are given by (7.1.3).

Factoring $\|\mathcal{H}(\delta)\| \frac{\partial\|\mathcal{H}(\delta)\|}{\partial \delta}$ from equation (7.1.16) and $\|\hat{\mathcal{H}}(\delta)\| \frac{\partial\|\hat{\mathcal{H}}(\delta)\|}{\partial \delta}$ from equation (7.1.17) gives us

$$
\begin{aligned}
& \frac{\partial t_{s}(\delta)}{\partial \delta}= \\
& -\|\mathcal{H}(\delta)\| \frac{\partial\|\mathcal{H}(\delta)\|}{\partial \delta}\left[\frac{2\left(\frac{-\mathcal{A}}{2}+\sqrt{\mathcal{F}(\delta)}\right)}{\left(\|\mathcal{H}(\delta)\|^{2}+\gamma_{s} \bar{\gamma}_{s}\right)^{2}}+\frac{\alpha}{\sqrt{\mathcal{F}}(\delta)\left(\|\mathcal{H}(\delta)\|^{2}+\gamma_{s} \bar{\gamma}_{s}\right)}\right]
\end{aligned}
$$




$$
\begin{aligned}
& \frac{\partial \hat{t}_{s}(\delta)}{\partial \delta}= \\
& -\|\hat{\mathcal{H}}(\delta)\| \frac{\partial\|\hat{\mathcal{H}}(\delta)\|}{\partial \delta}\left[\frac{2\left(\frac{-\mathcal{A}}{2}+\sqrt{\hat{\mathcal{F}}(\delta)}\right)}{\left(\|\hat{\mathcal{H}}(\delta)\|^{2}+\gamma_{s} \bar{\gamma}_{s}\right)^{2}}+\frac{\alpha}{\sqrt{\hat{\mathcal{F}}(\delta)}\left(\|\hat{\mathcal{H}}(\delta)\|^{2}+\gamma_{s} \bar{\gamma}_{s}\right)}\right] .
\end{aligned}
$$

We create the common denominator $\sqrt{\mathcal{F}}(\delta)\left(\|\mathcal{H}(\delta)\|^{2}+\gamma_{s} \bar{\gamma}_{s}\right)^{2}$ in (7.1.18) to yield

$$
\frac{\partial t_{s}(\delta)}{\partial \delta}=-\|\mathcal{H}(\delta)\| \frac{\partial\|\mathcal{H}(\delta)\|}{\partial \delta}\left[\frac{-\mathcal{A} \sqrt{\mathcal{F}(\delta)}+2 \mathcal{F}(\delta)+\alpha\left(\|\mathcal{H}(\delta)\|^{2}+\gamma_{s} \bar{\gamma}_{s}\right)}{\sqrt{\mathcal{F}(\delta)}\left(\|\mathcal{H}(\delta)\|^{2}+\gamma_{s} \bar{\gamma}_{s}\right)^{2}}\right] .
$$

Note that

$$
\alpha\left(\|\mathcal{H}(\delta)\|^{2}+\gamma_{s} \bar{\gamma}_{s}\right)=\frac{\mathcal{A}^{2}}{4}-\mathcal{F}(\delta) .
$$

Substituting the expression in equation (7.1.21) into equation (7.1.20) leaves us with

$$
\begin{aligned}
\frac{\partial t_{s}(\delta)}{\partial \delta} & =-\|\mathcal{H}(\delta)\| \frac{\partial\|\mathcal{H}(\delta)\|}{\partial \delta}\left[\frac{-\mathcal{A} \sqrt{\mathcal{F}(\delta)}+2 \mathcal{F}(\delta)+\frac{\mathcal{A}^{2}}{4}-\mathcal{F}(\delta)}{\sqrt{\mathcal{F}(\delta)}\left(\|\mathcal{H}(\delta)\|^{2}+\gamma_{s} \bar{\gamma}_{s}\right)^{2}}\right] \\
& =-\|\mathcal{H}(\delta)\| \frac{\partial\|\mathcal{H}(\delta)\|}{\partial \delta}\left[\frac{\frac{\mathcal{A}^{2}}{4}-\mathcal{A} \sqrt{\mathcal{F}(\delta)}+\mathcal{F}(\delta)}{\sqrt{\mathcal{F}(\delta)}\left(\|\mathcal{H}(\delta)\|^{2}+\gamma_{s} \bar{\gamma}_{s}\right)^{2}}\right] .
\end{aligned}
$$

Noticing the perfect square in the numerator of equation (7.1.22), we obtain

$$
\begin{aligned}
\frac{\partial t_{s}(\delta)}{\partial \delta} & =-\|\mathcal{H}(\delta)\| \frac{\partial\|\mathcal{H}(\delta)\|}{\partial \delta}\left[\frac{\left(\frac{-\mathcal{A}}{2}+\sqrt{\mathcal{F}(\delta)}\right)^{2}}{\sqrt{\mathcal{F}(\delta)}\left(\|\mathcal{H}(\delta)\|^{2}+\gamma_{s} \bar{\gamma}_{s}\right)^{2}}\right] \\
& =-\|\mathcal{H}(\delta)\| \frac{\partial\|\mathcal{H}(\delta)\|}{\partial \delta} \frac{\left(t_{s}(\delta)\right)^{2}}{\sqrt{\mathcal{F}(\delta)}} .
\end{aligned}
$$

Returning to the derivative of $Z(\delta)$, we see that

$$
\begin{aligned}
\frac{\partial Z(\delta)}{\partial \delta} & =\left(-\gamma_{s} \frac{\partial t_{s}(\delta)}{\partial \delta} u, \frac{\partial t_{s}(\delta)}{\partial \delta} \mathcal{H}(\delta)+\frac{\partial \mathcal{H}(\delta)}{\partial \delta} t_{s}(\delta)\right) \\
& =\left(-\gamma_{s} \frac{\partial t_{s}(\delta)}{\partial \delta} u, \frac{\partial t_{s}(\delta)}{\partial \delta}\left(q_{0}+\delta \mathcal{V}\right)+\mathcal{V} t_{s}(\delta)\right)
\end{aligned}
$$


Evaluate $\frac{\partial Z(\delta)}{\partial \delta}$ at $\delta=0$ and get

$$
\left.\frac{\partial Z(\delta)}{\partial \delta}\right|_{\delta=0}=\left(-\left.\gamma_{s} \frac{\partial t_{s}(\delta)}{\partial \delta}\right|_{\delta=0} u,\left.\frac{\partial t_{s}(\delta)}{\partial \delta}\right|_{\delta=0} q_{0}+\mathcal{V} t_{s}(0)\right)
$$

where

$$
\left.\frac{\partial t_{s}(\delta)}{\partial \delta}\right|_{\delta=0}=-\left.\|\mathcal{H}(0)\| \frac{\partial\|\mathcal{H}(\delta)\|}{\partial \delta}\right|_{\delta=0} \frac{\left(t_{s}(0)\right)^{2}}{\sqrt{\mathcal{F}(0)}}
$$

with

$$
\left.\frac{\partial\|\mathcal{H}(\delta)\|}{\partial \delta}\right|_{\delta=0}=\frac{\left\langle q_{0}, \mathcal{V}\right\rangle+\left\langle\mathcal{V}, q_{0}\right\rangle}{2\left\|q_{0}\right\|} \quad \text { and }\|\mathcal{H}(0)\|=\left\|q_{0}\right\| .
$$

Therefore

$$
\begin{aligned}
& \left.\frac{\partial Z(\delta)}{\partial \delta}\right|_{\delta=0}= \\
& \left(\gamma_{s} \frac{\left\langle q_{0}, \mathcal{V}\right\rangle+\left\langle\mathcal{V}, q_{0}\right\rangle}{2} \frac{\left(t_{s}(0)\right)^{2}}{\sqrt{\mathcal{F}(0)}} u,-\frac{\left\langle q_{0}, \mathcal{V}\right\rangle+\left\langle\mathcal{V}, q_{0}\right\rangle}{2} \frac{\left(t_{s}(0)\right)^{2}}{\sqrt{\mathcal{F}(0)}} q_{0}+\mathcal{V} t_{s}(0)\right)
\end{aligned}
$$

Similarly,

$$
\begin{aligned}
& \left.\frac{\partial \hat{Z}(\delta)}{\partial \delta}\right|_{\delta=0}= \\
& \left(\gamma_{s} \frac{\left\langle q_{0}, \hat{\mathcal{V}}\right\rangle+\left\langle\hat{\mathcal{V}}, q_{0}\right\rangle}{2} \frac{\left(\hat{t}_{s}(0)\right)^{2}}{\sqrt{\mathcal{F}(0)}} u,-\frac{\left\langle q_{0}, \hat{\mathcal{V}}\right\rangle+\left\langle\hat{\mathcal{V}}, q_{0}\right\rangle}{2} \frac{\left(\hat{t}_{s}(0)\right)^{2}}{\sqrt{\mathcal{F}(0)}} q_{0}+\hat{\mathcal{V}} \hat{t}_{s}(0)\right)
\end{aligned}
$$

Note that

$$
\left.\frac{\partial \hat{t}_{s}(\delta)}{\partial \delta}\right|_{\delta=0}=-\left.\|\hat{\mathcal{H}}(\delta)\| \frac{\partial\|\hat{\mathcal{H}}(\delta)\|}{\partial \delta}\right|_{\delta=0}\left[\frac{\left(\hat{t}_{s}(0)\right)^{2}}{\sqrt{\mathcal{F}(0)}}\right]
$$

with

$$
\left.\frac{\partial \hat{\mathcal{H}}(\delta)}{\partial \delta}\right|_{\delta=0}=\frac{\left\langle q_{0}, \hat{\mathcal{V}}\right\rangle+\left\langle\hat{\mathcal{V}}, q_{0}\right\rangle}{2\left\|q_{0}\right\|} \quad \text { and }\|\hat{\mathcal{H}}(0)\|=\left\|q_{0}\right\| .
$$

In order to determine the image of the angle $\mathcal{X}$ we calculate

$$
\cos (\mathcal{Y})=\left.\frac{\left\langle\frac{\partial Z(\delta)}{\partial \delta}, \frac{\partial \hat{Z}(\delta)}{\partial \delta}\right\rangle_{H_{N}}+\left\langle\frac{\partial \hat{Z}(\delta)}{\partial \delta}, \frac{\partial Z(\delta)}{\partial \delta}\right\rangle_{H_{N}}}{2\left\|\frac{\partial Z(\delta)}{\partial \delta}\right\|_{H_{N}}\left\|\frac{\partial \hat{Z}(\delta)}{\partial \delta}\right\|_{H_{N}}}\right|_{\delta=0} .
$$


To this end we first calculate the numerator of (7.1.26) that requires the evaluation of $\left.\frac{\partial Z(\delta)}{\partial \delta}\right|_{\delta=0}$ and $\left.\frac{\partial \hat{Z}(\delta)}{\partial \delta}\right|_{\delta=0}$ given by equations (7.1.24) and (7.1.25). Therefore we have

$$
\begin{aligned}
& \left.\left\langle\frac{\partial Z(\delta)}{\partial \delta}, \frac{\partial \hat{Z}(\delta)}{\partial \delta}\right\rangle_{H_{N}}\right|_{\delta=0} \\
& =\left\langle\gamma_{s} \frac{\left\langle q_{0}, \mathcal{V}\right\rangle+\left\langle\mathcal{V}, q_{0}\right\rangle}{2} \frac{\left(t_{s}(0)\right)^{2}}{\sqrt{\mathcal{F}(0)}} u, \gamma_{s} \frac{\left\langle q_{0}, \hat{\mathcal{V}}\right\rangle+\left\langle\hat{\mathcal{V}}, q_{0}\right\rangle}{2} \frac{\left(\hat{t}_{s}(0)\right)^{2}}{\sqrt{\mathcal{F}(0)}} u\right\rangle+ \\
& \left\langle\frac{\left\langle q_{0}, \mathcal{V}\right\rangle+\left\langle\mathcal{V}, q_{0}\right\rangle}{2} \frac{\left(t_{s}(0)\right)^{2}}{\sqrt{\mathcal{F}(0)}} q_{0}+\mathcal{V} t_{s}(0), \frac{\left\langle q_{0}, \hat{\mathcal{V}}\right\rangle+\left\langle\hat{\mathcal{V}}, q_{0}\right\rangle}{2} \frac{\left(\hat{t}_{s}(0)\right)^{2}}{\sqrt{\mathcal{F}(0)}} q_{0}+\hat{\mathcal{V}} \hat{t}_{s}(0)\right\rangle \\
& =\gamma_{s} \bar{\gamma}_{s}\left(t_{s}(0)\right)^{2}\left(\hat{t}_{s}(0)\right)^{2} \frac{\left[\left\langle q_{0}, \mathcal{V}\right\rangle+\left\langle\mathcal{V}, q_{0}\right\rangle\right]\left[\overline{\left\langle q_{0}, \hat{\mathcal{V}}\right\rangle}+\overline{\left\langle\hat{\mathcal{V}}, q_{0}\right\rangle}\right]}{4 \mathcal{F}(0)}\langle u, u\rangle \\
& +\left\langle-\frac{\left\langle q_{0}, \mathcal{V}\right\rangle+\left\langle\mathcal{V}, q_{0}\right\rangle}{2} \frac{\left(t_{s}(0)\right)^{2}}{\sqrt{\mathcal{F}(0)}} q_{0},-\frac{\left\langle q_{0}, \hat{\mathcal{V}}\right\rangle+\left\langle\hat{\mathcal{V}}, q_{0}\right\rangle}{2} \frac{\left(\hat{t}_{s}(0)\right)^{2}}{\sqrt{\mathcal{F}(0)}} q_{0}\right\rangle \\
& +\left\langle-\frac{\left\langle q_{0}, \mathcal{V}\right\rangle+\left\langle\mathcal{V}, q_{0}\right\rangle}{2} \frac{\left(t_{s}(0)\right)^{2}}{\sqrt{\mathcal{F}(0)}} q_{0}, \hat{\mathcal{V}} \hat{t}_{s}(0)\right\rangle \\
& +\left\langle\mathcal{V} t_{s}(0),-\frac{\left\langle q_{0}, \hat{\mathcal{V}}\right\rangle+\left\langle\hat{\mathcal{V}}, q_{0}\right\rangle}{2} \frac{\left(\hat{t}_{s}(0)\right)^{2}}{\sqrt{\mathcal{F}(0)}} q_{0}\right\rangle+\left\langle\mathcal{V} t_{s}(0), \hat{\mathcal{V}} \hat{t}_{s}(0)\right\rangle \\
& =\gamma_{s} \bar{\gamma}_{s}\left(t_{s}(0)\right)^{2}\left(\hat{t}_{s}(0)\right)^{2} \frac{\left[\left\langle q_{0}, \mathcal{V}\right\rangle+\left\langle\mathcal{V}, q_{0}\right\rangle\right]\left[\overline{\left\langle q_{0}, \hat{\mathcal{V}}\right\rangle}+\overline{\left\langle\hat{\mathcal{V}}, q_{0}\right\rangle}\right]}{4 \mathcal{F}(0)} \\
& +\left(t_{s}(0)\right)^{2}\left(\hat{t}_{s}(0)\right)^{2} \frac{\left[\left\langle q_{0}, \mathcal{V}\right\rangle+\left\langle\mathcal{V}, q_{0}\right\rangle\right]\left[\overline{\left\langle q_{0}, \hat{\mathcal{V}}\right\rangle}+\overline{\left\langle\hat{\mathcal{V}}, q_{0}\right\rangle}\right]}{4 \mathcal{F}(0)}\left\langle q_{0}, q_{0}\right\rangle \\
& -\left(t_{s}(0)\right)^{2} \hat{t}_{s}(0) \frac{\left\langle q_{0}, \mathcal{V}\right\rangle+\left\langle\mathcal{V}, q_{0}\right\rangle}{2 \sqrt{\mathcal{F}(0)}}\left\langle q_{0}, \hat{\mathcal{V}}\right\rangle \\
& -t_{s}(0)\left(\hat{t}_{s}(0)\right)^{2} \frac{\overline{\left\langle q_{0}, \hat{\mathcal{V}}\right\rangle}+\overline{\left\langle\hat{\mathcal{V}}, q_{0}\right\rangle}}{2 \sqrt{\mathcal{F}(0)}}\left\langle\mathcal{V}, q_{0}\right\rangle+t_{s}(0) \hat{t}_{s}(0)\langle\mathcal{V}, \hat{\mathcal{V}}\rangle=
\end{aligned}
$$




$$
\begin{aligned}
& =\gamma_{s} \bar{\gamma}_{s}\left(t_{s}(0)\right)^{4} \frac{\left[\left\langle q_{0}, \mathcal{V}\right\rangle+\left\langle\mathcal{V}, q_{0}\right\rangle\right]\left[\overline{\left\langle q_{0}, \hat{\mathcal{V}}\right\rangle}+\overline{\left\langle\hat{\mathcal{V}}, q_{0}\right\rangle}\right]}{4 \mathcal{F}(0)} \\
& +\left(t_{s}(0)\right)^{4} \frac{\left[\left\langle q_{0}, \mathcal{V}\right\rangle+\left\langle\mathcal{V}, q_{0}\right\rangle\right]\left[\overline{\left\langle q_{0}, \hat{\mathcal{V}}\right\rangle}+\overline{\left\langle\hat{\mathcal{V}}, q_{0}\right\rangle}\right]}{4 \mathcal{F}(0)}\left\langle q_{0}, q_{0}\right\rangle \\
& -\left(t_{s}(0)\right)^{3} \frac{\left\langle q_{0}, \mathcal{V}\right\rangle+\left\langle\mathcal{V}, q_{0}\right\rangle}{2 \sqrt{\mathcal{F}(0)}}\left\langle q_{0}, \hat{\mathcal{V}}\right\rangle \\
& -\left(t_{s}(0)\right)^{3} \frac{\overline{\left\langle q_{0}, \hat{\mathcal{V}}\right\rangle}+\overline{\left\langle\hat{\mathcal{V}}, q_{0}\right\rangle}}{2 \sqrt{\mathcal{F}(0)}}\left\langle\mathcal{V}, q_{0}\right\rangle+\left(t_{s}(0)\right)^{2}\langle\mathcal{V}, \hat{\mathcal{V}}\rangle . \\
& \left.\left\langle\frac{\partial Z(\delta)}{\partial \delta}, \frac{\partial \hat{Z}(\delta)}{\partial \delta}\right\rangle_{H_{N}}\right|_{\delta=0} \\
& =\left(t_{s}(0)\right)^{4} \frac{\left[\left\langle q_{0}, \mathcal{V}\right\rangle+\left\langle\mathcal{V}, q_{0}\right\rangle\right]\left[\overline{\left\langle q_{0}, \hat{\mathcal{V}}\right\rangle}+\overline{\left\langle\hat{\mathcal{V}}, q_{0}\right\rangle}\right]}{4 \mathcal{F}(0)}\left(\gamma_{s} \bar{\gamma}_{s}+\left\|q_{0}\right\|\right) \\
& -\left(t_{s}(0)\right)^{3} \frac{\left\langle q_{0}, \mathcal{V}\right\rangle+\left\langle\mathcal{V}, q_{0}\right\rangle}{2 \sqrt{\mathcal{F}(0)}}\left\langle q_{0}, \hat{\mathcal{V}}\right\rangle \\
& -\left(t_{s}(0)\right)^{3} \frac{\overline{\left\langle q_{0}, \hat{\mathcal{V}}\right\rangle}+\overline{\left\langle\hat{\mathcal{V}}, q_{0}\right\rangle}}{2 \sqrt{\mathcal{F}(0)}}\left\langle\mathcal{V}, q_{0}\right\rangle+\left(t_{s}(0)\right)^{2}\langle\mathcal{V}, \hat{\mathcal{V}}\rangle .
\end{aligned}
$$

Similarly,

$$
\begin{aligned}
& \left.\left\langle\frac{\partial \hat{Z}(\delta)}{\partial \delta}, \frac{\partial Z(\delta)}{\partial \delta}\right\rangle_{H_{N}}\right|_{\delta=0} \\
& =\left(t_{s}(0)\right)^{4} \frac{\left[\left\langle q_{0}, \hat{\mathcal{V}}\right\rangle+\left\langle\hat{\mathcal{V}}, q_{0}\right\rangle\right]\left[\overline{\left\langle q_{0}, \mathcal{V}\right\rangle}+\overline{\left\langle\mathcal{V}, q_{0}\right\rangle}\right]}{4 \mathcal{F}(0)}\left(\gamma_{s} \bar{\gamma}_{s}+\left\|q_{0}\right\|\right) \\
& -\left(t_{s}(0)\right)^{3} \frac{\left\langle q_{0}, \hat{\mathcal{V}}\right\rangle+\left\langle\hat{\mathcal{V}}, q_{0}\right\rangle}{2 \sqrt{\mathcal{F}(0)}}\left\langle q_{0}, \mathcal{V}\right\rangle \\
& -\left(t_{s}(0)\right)^{3} \frac{\frac{\left\langle q_{0}, \mathcal{V}\right\rangle}{\left\langle\sqrt{\left.\mathcal{V}, q_{0}\right\rangle}\right.}}{2 \sqrt{\mathcal{F}(0)}}\left\langle\hat{\mathcal{V}}, q_{0}\right\rangle+\left(t_{s}(0)\right)^{2}\langle\hat{\mathcal{V}}, \mathcal{V}\rangle .
\end{aligned}
$$

Add equations (7.1.27) and (7.1.28) to get 


$$
\begin{aligned}
& \left.\left\langle\frac{\partial Z(\delta)}{\partial \delta}, \frac{\partial \hat{Z}(\delta)}{\partial \delta}\right\rangle_{H_{N}}\right|_{\delta=0}+\left.\left\langle\frac{\partial \hat{Z}(\delta)}{\partial \delta}, \frac{\partial Z(\delta)}{\partial \delta}\right\rangle_{H_{N}}\right|_{\delta=0} \\
& =\left(t_{s}(0)\right)^{4} \frac{\left[\left\langle q_{0}, \mathcal{V}\right\rangle+\left\langle\mathcal{V}, q_{0}\right\rangle\right]\left[\overline{\left\langle q_{0}, \hat{\mathcal{V}}\right\rangle}+\overline{\left\langle\hat{\mathcal{V}}, q_{0}\right\rangle}\right]}{4 \mathcal{F}(0)}\left(\gamma_{s} \bar{\gamma}_{s}+\left\|q_{0}\right\|\right) \\
& +\left(t_{s}(0)\right)^{4} \frac{\left[\left\langle q_{0}, \hat{\mathcal{V}}\right\rangle+\left\langle\hat{\mathcal{V}}, q_{0}\right\rangle\right]\left[\overline{\left\langle q_{0}, \mathcal{V}\right\rangle}+\overline{\left\langle\mathcal{V}, q_{0}\right\rangle}\right]}{4 \mathcal{F}(0)}\left(\gamma_{s} \bar{\gamma}_{s}+\left\|q_{0}\right\|\right) \\
& -\left(t_{s}(0)\right)^{3} \frac{\left\langle q_{0}, \mathcal{V}\right\rangle+\left\langle\mathcal{V}, q_{0}\right\rangle}{2 \sqrt{\mathcal{F}(0)}}\left\langle q_{0}, \hat{\mathcal{V}}\right\rangle-\left(t_{s}(0)\right)^{3} \frac{\overline{\left\langle q_{0}, \mathcal{V}\right\rangle}+\overline{\left\langle\mathcal{V}, q_{0}\right\rangle}}{2 \sqrt{\mathcal{F}(0)}}\left\langle\hat{\mathcal{V}}, q_{0}\right\rangle \\
& -\left(t_{s}(0)\right)^{3} \frac{\overline{\left\langle q_{0}, \hat{\mathcal{V}}\right\rangle}+\overline{\left\langle\hat{\mathcal{V}}, q_{0}\right\rangle}}{2 \sqrt{\mathcal{F}(0)}}\left\langle\mathcal{V}, q_{0}\right\rangle-\left(t_{s}(0)\right)^{3} \frac{\left\langle q_{0}, \hat{\mathcal{V}}\right\rangle+\left\langle\hat{\mathcal{V}}, q_{0}\right\rangle}{2 \sqrt{\mathcal{F}(0)}}\left\langle q_{0}, \mathcal{V}\right\rangle \\
& +\left(t_{s}(0)\right)^{2}\langle\mathcal{V}, \hat{\mathcal{V}}\rangle+\left(t_{s}(0)\right)^{2}\langle\hat{\mathcal{V}}, \mathcal{V}\rangle \\
& =\left(t_{s}(0)\right)^{4} \frac{\left[\left\langle q_{0}, \mathcal{V}\right\rangle+\left\langle\mathcal{V}, q_{0}\right\rangle\right]\left[\overline{\left\langle q_{0}, \hat{\mathcal{V}}\right\rangle}+\overline{\left\langle\hat{\mathcal{V}}, q_{0}\right\rangle}\right]}{4 \mathcal{F}(0)}\left(\gamma_{s} \bar{\gamma}_{s}+\left\|q_{0}\right\|\right) \\
& +\left(t_{s}(0)\right)^{4} \frac{\left[\left\langle q_{0}, \hat{\mathcal{V}}\right\rangle+\left\langle\hat{\mathcal{V}}, q_{0}\right\rangle\right]\left[\overline{\left\langle q_{0}, \mathcal{V}\right\rangle}+\overline{\left\langle\mathcal{V}, q_{0}\right\rangle}\right]}{4 \mathcal{F}(0)}\left(\gamma_{s} \bar{\gamma}_{s}+\left\|q_{0}\right\|\right) \\
& -\left(t_{s}(0)\right)^{3} \frac{\overline{\left\langle q_{0}, \mathcal{V}\right\rangle}+\overline{\left\langle\mathcal{V}, q_{0}\right\rangle}}{2 \sqrt{\mathcal{F}(0)}}\left\langle q_{0}, \hat{\mathcal{V}}\right\rangle-\left(t_{s}(0)\right)^{3} \frac{\overline{\left\langle q_{0}, \mathcal{V}\right\rangle}+\overline{\left\langle\mathcal{V}, q_{0}\right\rangle}}{2 \sqrt{\mathcal{F}(0)}}\left\langle\hat{\mathcal{V}}, q_{0}\right\rangle \\
& -\left(t_{s}(0)\right)^{3} \frac{\overline{\left\langle q_{0}, \hat{\mathcal{V}}\right\rangle}+\overline{\left\langle\hat{\mathcal{V}}, q_{0}\right\rangle}}{2 \sqrt{\mathcal{F}(0)}}\left\langle\mathcal{V}, q_{0}\right\rangle-\left(t_{s}(0)\right)^{3} \frac{\overline{\left\langle q_{0}, \hat{\mathcal{V}}\right\rangle}+\overline{\left\langle\hat{\mathcal{V}}, q_{0}\right\rangle}}{2 \sqrt{\mathcal{F}(0)}}\left\langle q_{0}, \mathcal{V}\right\rangle \\
& +\left(t_{s}(0)\right)^{2}\langle\mathcal{V}, \hat{\mathcal{V}}\rangle+\left(t_{s}(0)\right)^{2}\langle\hat{\mathcal{V}}, \mathcal{V}\rangle
\end{aligned}
$$




$$
\begin{aligned}
& =\left(t_{s}(0)\right)^{4} \frac{\left[\left\langle q_{0}, \mathcal{V}\right\rangle+\left\langle\mathcal{V}, q_{0}\right\rangle\right]\left[\overline{\left\langle q_{0}, \hat{\mathcal{V}}\right\rangle}+\overline{\left\langle\hat{\mathcal{V}}, q_{0}\right\rangle}\right]}{4 \mathcal{F}(0)}\left(\gamma_{s} \bar{\gamma}_{s}+\left\|q_{0}\right\|\right) \\
& +\left(t_{s}(0)\right)^{4} \frac{\left[\left\langle q_{0}, \hat{\mathcal{V}}\right\rangle+\left\langle\hat{\mathcal{V}}, q_{0}\right\rangle\right]\left[\overline{\left\langle q_{0}, \mathcal{V}\right\rangle}+\overline{\left\langle\mathcal{V}, q_{0}\right\rangle}\right]}{4 \mathcal{F}(0)}\left(\gamma_{s} \bar{\gamma}_{s}+\left\|q_{0}\right\|\right) \\
& -\frac{\left(t_{s}(0)\right)^{3}}{2 \sqrt{\mathcal{F}(0)}}\left[\left\langle q_{0}, \hat{\mathcal{V}}\right\rangle+\left\langle\hat{\mathcal{V}}, q_{0}\right\rangle\right]\left[\overline{\left\langle q_{0}, \mathcal{V}\right\rangle}+\overline{\left\langle\mathcal{V}, q_{0}\right\rangle}\right] \\
& -\frac{\left(t_{s}(0)\right)^{3}}{2 \sqrt{\mathcal{F}(0)}}\left[\left\langle q_{0}, \mathcal{V}\right\rangle+\left\langle\mathcal{V}, q_{0}\right\rangle\right]\left[\overline{\left\langle q_{0}, \hat{\mathcal{V}}\right\rangle}+\overline{\left\langle\hat{\mathcal{V}}, q_{0}\right\rangle}\right] \\
& +\left(t_{s}(0)\right)^{2}\langle\mathcal{V}, \hat{\mathcal{V}}\rangle+\left(t_{s}(0)\right)^{2}\langle\hat{\mathcal{V}}, \mathcal{V}\rangle .
\end{aligned}
$$

Note that

$$
\begin{aligned}
& {\left[\left\langle q_{0}, \mathcal{V}\right\rangle+\left\langle\mathcal{V}, q_{0}\right\rangle\right]\left[\overline{\left\langle q_{0}, \hat{\mathcal{V}}\right\rangle}+\overline{\left\langle\hat{\mathcal{V}}, q_{0}\right\rangle}\right]+\left[\left\langle q_{0}, \hat{\mathcal{V}}\right\rangle+\left\langle\hat{\mathcal{V}}, q_{0}\right\rangle\right]\left[\overline{\left\langle q_{0}, \mathcal{V}\right\rangle}+\overline{\left\langle\mathcal{V}, q_{0}\right\rangle}\right]} \\
& = \\
& {\left[\left\langle q_{0}, \mathcal{V}\right\rangle+\left\langle\mathcal{V}, q_{0}\right\rangle\right]\left[\left\langle q_{0}, \hat{\mathcal{V}}\right\rangle+\left\langle\hat{\mathcal{V}}, q_{0}\right\rangle\right]+\left[\left\langle q_{0}, \hat{\mathcal{V}}\right\rangle+\left\langle\hat{\mathcal{V}}, q_{0}\right\rangle\right]\left[\left\langle q_{0}, \mathcal{V}\right\rangle+\left\langle\mathcal{V}, q_{0}\right\rangle\right]} \\
& =2\left[\left\langle q_{0}, \mathcal{V}\right\rangle+\left\langle\mathcal{V}, q_{0}\right\rangle\right]\left[\left\langle q_{0}, \hat{\mathcal{V}}\right\rangle+\left\langle\hat{\mathcal{V}}, q_{0}\right\rangle\right] .
\end{aligned}
$$

Thus

$$
\begin{aligned}
& \left.\left\langle\frac{\partial Z(\delta)}{\partial \delta}, \frac{\partial \hat{Z}(\delta)}{\partial \delta}\right\rangle_{H_{N}}\right|_{\delta=0}+\left.\left\langle\frac{\partial \hat{Z}(\delta)}{\partial \delta}, \frac{\partial Z(\delta)}{\partial \delta}\right\rangle_{H_{N}}\right|_{\delta=0} \\
& =\frac{\left(t_{s}(0)\right)^{4}}{2 \mathcal{F}(0)}\left(\left\|q_{0}\right\|+\gamma_{s} \bar{\gamma}_{s}\right)\left[\left\langle q_{0}, \mathcal{V}\right\rangle+\left\langle\mathcal{V}, q_{0}\right\rangle\right]\left[\left\langle q_{0}, \hat{\mathcal{V}}\right\rangle+\left\langle\hat{\mathcal{V}}, q_{0}\right\rangle\right] \\
& -\frac{\left(t_{s}(0)\right)^{3}}{\sqrt{\mathcal{F}(0)}}\left[\left\langle q_{0}, \mathcal{V}\right\rangle+\left\langle\mathcal{V}, q_{0}\right\rangle\right]\left[\left\langle q_{0}, \hat{\mathcal{V}}\right\rangle+\left\langle\hat{\mathcal{V}}, q_{0}\right\rangle\right]+\left(t_{s}(0)\right)^{2}[\langle\mathcal{V}, \hat{\mathcal{V}}\rangle+\langle\hat{\mathcal{V}}, \mathcal{V}\rangle]
\end{aligned}
$$

Factoring $\left(t_{s}(0)\right)^{2}\left[\left\langle q_{0}, \mathcal{V}\right\rangle+\left\langle\mathcal{V}, q_{0}\right\rangle\right]\left[\left\langle q_{0}, \hat{\mathcal{V}}\right\rangle+\left\langle\hat{\mathcal{V}}, q_{0}\right\rangle\right]$ from first and second 
terms in the equation (7.1.29) leaves us with

$$
\begin{aligned}
& \left.\left\langle\frac{\partial Z(\delta)}{\partial \delta}, \frac{\partial \hat{Z}(\delta)}{\partial \delta}\right\rangle_{H_{N}}\right|_{\delta=0}+\left.\left\langle\frac{\partial \hat{Z}(\delta)}{\partial \delta}, \frac{\partial Z(\delta)}{\partial \delta}\right\rangle_{H_{N}}\right|_{\delta=0}=\left(t_{s}(0)\right)^{2}\{ \\
& \left.\frac{\left[\left\langle q_{0}, \mathcal{V}\right\rangle+\left\langle\mathcal{V}, q_{0}\right\rangle\right]\left[\left\langle q_{0}, \hat{\mathcal{V}}\right\rangle+\left\langle\hat{\mathcal{V}}, q_{0}\right\rangle\right]}{\sqrt{\mathcal{F}(0)}}\left[\frac{\left(t_{s}(0)\right)^{2}}{2 \sqrt{\mathcal{F}(0)}}\left(\left\|q_{0}\right\|^{2}+\gamma_{s} \bar{\gamma}_{s}\right)-t_{s}(0)\right]\right\} \\
& +\left(t_{s}(0)\right)^{2}[\langle\mathcal{V}, \hat{\mathcal{V}}\rangle+\langle\hat{\mathcal{V}}, \mathcal{V}\rangle] .
\end{aligned}
$$

We examine $\frac{\left(t_{s}(0)\right)^{2}}{2 \sqrt{\mathcal{F}(0)}}\left(\left\|q_{0}\right\|^{2}+\gamma_{s} \bar{\gamma}_{s}\right)-t_{s}(0)$. Expanding $\left(t_{s}(0)\right)^{2}$ we have

$$
\frac{\left(t_{s}(0)\right)^{2}}{2 \sqrt{\mathcal{F}(0)}}\left(\left\|q_{0}\right\|^{2}+\gamma_{s} \bar{\gamma}_{s}\right)-t_{s}(0)=\frac{t_{s}(0)\left(\left\|q_{0}\right\|^{2}+\gamma_{s} \bar{\gamma}_{s}\right)}{2 \sqrt{\mathcal{F}(0)}} t_{s}(0)-t_{s}(0)
$$

Recalling the definition of $t_{s}(0)$ from equation (7.2.6), we are left with

$$
\begin{aligned}
\frac{t_{s}(0)\left(\left\|q_{0}\right\|^{2}+\gamma_{s} \bar{\gamma}_{s}\right)}{\sqrt{\mathcal{F}(0)}} t_{s}(0)-t_{s}(0) & =\frac{\frac{-\mathcal{A}}{2}+\sqrt{\mathcal{F}(0)}}{2 \sqrt{\mathcal{F}(0)}} t_{s}(0)-t_{s}(0) \\
& =t_{s}(0)\left(\frac{\frac{-\mathcal{A}}{2}+\sqrt{\mathcal{F}(0)}}{2 \sqrt{\mathcal{F}(0)}}-1\right)
\end{aligned}
$$

Setting a common denominator in equation(7.1.31) gives us

$$
\begin{aligned}
t_{s}(0)\left(\frac{\frac{-\mathcal{A}}{2}+\sqrt{\mathcal{F}(0)}}{2 \sqrt{\mathcal{F}(0)}}-1\right) & =t_{s}(0)\left(\frac{\frac{-\mathcal{A}}{2}+\sqrt{\mathcal{F}(0)}-2 \sqrt{\mathcal{F}(0)}}{2 \sqrt{\mathcal{F}(0)}}\right) \\
& =t_{s}(0)\left(\frac{\frac{-\mathcal{A}}{2}-\sqrt{\mathcal{F}(0)}}{2 \sqrt{\mathcal{F}(0)}}\right)
\end{aligned}
$$

Substituting $t_{s}(0)$ as expressed in equation (7.1.14) into equation (7.1.32), we have

$$
\begin{aligned}
t_{s}(0)\left(\frac{\frac{-\mathcal{A}}{2}-\sqrt{\mathcal{F}(0)}}{2 \sqrt{\mathcal{F}(0)}}\right) & =\frac{\frac{-\mathcal{A}}{2}+\sqrt{\mathcal{F}(0)}}{\left\|q_{0}\right\|^{2}+\gamma_{s} \bar{\gamma}_{s}}\left(\frac{\frac{-\mathcal{A}}{2}-\sqrt{\mathcal{F}(0)}}{2 \sqrt{\mathcal{F}(0)}}\right) \\
& =\frac{\frac{\mathcal{A}^{2}}{4}-\mathcal{F}(0)}{2 \sqrt{\mathcal{F}(0)}\left(\left\|q_{0}\right\|^{2}+\gamma_{s} \bar{\gamma}_{s}\right)}
\end{aligned}
$$


Into equation (7.1.33) we substitute the expression for given in equation (7.1.14) to obtain

$$
\frac{\frac{\mathcal{A}^{2}}{4}-\mathcal{F}(0)}{2 \sqrt{\mathcal{F}(0)}\left(\left\|q_{0}\right\|^{2}+\gamma_{s} \bar{\gamma}_{s}\right)}=\frac{\frac{\mathcal{A}^{2}}{4}-\left(\frac{\mathcal{A}^{2}}{4}-\alpha\left(\left\|q_{0}\right\|^{2}+\gamma_{s} \bar{\gamma}_{s}\right)\right)}{2 \sqrt{\mathcal{F}(0)}\left(\left\|q_{0}\right\|^{2}+\gamma_{s} \bar{\gamma}_{s}\right)}=\frac{\alpha}{2 \sqrt{\mathcal{F}(0)}}
$$

therefore

$$
\frac{\left(t_{s}(0)\right)^{2}}{2 \sqrt{\mathcal{F}(0)}}\left(\left\|q_{0}\right\|^{2}+\gamma_{s} \bar{\gamma}_{s}\right)-t_{s}(0)=\frac{\alpha}{2 \sqrt{\mathcal{F}(0)}}
$$

Substituting the expression given in equation (7.1.34) into (7.1.30)

$$
\begin{aligned}
& \left.\left\langle\frac{\partial Z(\delta)}{\partial \delta}, \frac{\partial \hat{Z}(\delta)}{\partial \delta}\right\rangle_{H_{N}}\right|_{\delta=0}+\left.\left\langle\frac{\partial \hat{Z}(\delta)}{\partial \delta}, \frac{\partial Z(\delta)}{\partial \delta}\right\rangle_{H_{N}}\right|_{\delta=0} \\
& =\left(t_{s}(0)\right)^{2} \frac{\left[\left\langle q_{0}, \mathcal{V}\right\rangle+\left\langle\mathcal{V}, q_{0}\right\rangle\right]\left[\left\langle q_{0}, \hat{\mathcal{V}}\right\rangle+\left\langle\hat{\mathcal{V}}, q_{0}\right\rangle\right]}{\sqrt{\mathcal{F}(0)}}\left[\frac{\alpha}{2 \sqrt{\mathcal{F}(0)}}\right] \\
& +\left(t_{s}(0)\right)^{2}[\langle\mathcal{V}, \hat{\mathcal{V}}\rangle+\langle\hat{\mathcal{V}}, \mathcal{V}\rangle] \\
& =\frac{\left(t_{s}(0)\right)^{2}}{2 \mathcal{F}(0)} \alpha\left[\left\langle q_{0}, \mathcal{V}\right\rangle+\left\langle\mathcal{V}, q_{0}\right\rangle\right]\left[\left\langle q_{0}, \hat{\mathcal{V}}\right\rangle+\left\langle\hat{\mathcal{V}}, q_{0}\right\rangle\right]+\left(t_{s}(0)\right)^{2}[\langle\mathcal{V}, \hat{\mathcal{V}}\rangle+\langle\hat{\mathcal{V}}, \mathcal{V}\rangle]
\end{aligned}
$$

Factoring $\left(t_{s}(0)\right)^{2}$ from equation (7.1.35) and then substituting $\cos (\mathcal{X})$ and $\mathcal{J}$ expressed in equations (1.1.2) and (7.1.10) respectively, we see that

$$
\left.\left\langle\frac{\partial Z(\delta)}{\partial \delta}, \frac{\partial \hat{Z}(\delta)}{\partial \delta}\right\rangle_{H_{N}}\right|_{\delta=0}+\left.\left\langle\frac{\partial \hat{Z}(\delta)}{\partial \delta}, \frac{\partial Z(\delta)}{\partial \delta}\right\rangle_{H_{N}}\right|_{\delta=0}=2\left(t_{s}(0)\right)^{2}(\cos (\mathcal{X})+\mathcal{J})
$$

We now return to the denominator of $\cos (\mathcal{Y})$ in equation (7.1.26). Expanding 
the squared quantities and substituting as in equation (7.1.29), we have

$$
\begin{aligned}
& \left\|\frac{\partial Z(\delta)}{\partial \delta}\right\|_{\delta=0}^{2} \\
& =\left\langle\gamma_{s} \frac{\left\langle q_{0}, \mathcal{V}\right\rangle+\left\langle\mathcal{V}, q_{0}\right\rangle}{2} \frac{\left(t_{s}(0)\right)^{2}}{\sqrt{\mathcal{F}(0)}} u, \gamma_{s} \frac{\left\langle q_{0}, \mathcal{V}\right\rangle+\left\langle\mathcal{V}, q_{0}\right\rangle}{2} \frac{\left(t_{s}(0)\right)^{2}}{\sqrt{\mathcal{F}(0)}} u\right\rangle \\
& +\left\langle-\frac{\left\langle q_{0}, \mathcal{V}\right\rangle+\left\langle\mathcal{V}, q_{0}\right\rangle}{2} \frac{\left(t_{s}(0)\right)^{2}}{\sqrt{\mathcal{F}(0)}} q_{0}+\mathcal{V} t_{s}(0),-\frac{\left\langle q_{0}, \mathcal{V}\right\rangle+\left\langle\mathcal{V}, q_{0}\right\rangle}{2} \frac{\left(t_{s}(0)\right)^{2}}{\sqrt{\mathcal{F}(0)}} q_{0}\right. \\
& \left.+\mathcal{V} t_{s}(0)\right\rangle \\
& =\gamma_{s} \bar{\gamma}_{s} \frac{\left(t_{s}(0)\right)^{4}}{4 \mathcal{F}(0)}\left[\left\langle q_{0}, \mathcal{V}\right\rangle+\left\langle\mathcal{V}, q_{0}\right\rangle\right]\left[\overline{\left\langle q_{0}, \mathcal{V}\right\rangle}+\overline{\left\langle\mathcal{V}, q_{0}\right\rangle}\right]\langle u, u\rangle \\
& +\left\langle-\frac{\left\langle q_{0}, \mathcal{V}\right\rangle+\left\langle\mathcal{V}, q_{0}\right\rangle}{2} \frac{\left(t_{s}(0)\right)^{2}}{\sqrt{\mathcal{F}(0)}} q_{0},-\frac{\left\langle q_{0}, \mathcal{V}\right\rangle+\left\langle\mathcal{V}, q_{0}\right\rangle}{2} \frac{\left(t_{s}(0)\right)^{2}}{\sqrt{\mathcal{F}(0)}} q_{0}\right\rangle \\
& +\left\langle-\frac{\left\langle q_{0}, \mathcal{V}\right\rangle+\left\langle\mathcal{V}, q_{0}\right\rangle}{2} \frac{\left(t_{s}(0)\right)^{2}}{\sqrt{\mathcal{F}(0)}} q_{0}, \mathcal{V} t_{s}(0)\right\rangle \\
& +\left\langle\mathcal{V} t_{s}(0),-\frac{\left\langle q_{0}, \mathcal{V}\right\rangle+\left\langle\mathcal{V}, q_{0}\right\rangle}{2} \frac{\left(t_{s}(0)\right)^{2}}{\sqrt{\mathcal{F}(0)}} q_{0}\right\rangle+\left\langle\mathcal{V} t_{s}(0), \mathcal{V} t_{s}(0)\right\rangle \\
& =\gamma_{s} \bar{\gamma}_{s} \frac{\left(t_{s}(0)\right)^{4}}{4 \mathcal{F}(0)}\left[\left\langle q_{0}, \mathcal{V}\right\rangle+\left\langle\mathcal{V}, q_{0}\right\rangle\right]\left[\overline{\left\langle q_{0}, \mathcal{V}\right\rangle}+\overline{\left\langle\mathcal{V}, q_{0}\right\rangle}\right] \\
& +\frac{\left(t_{s}(0)\right)^{4}}{4 \mathcal{F}(0)}\left[\left\langle q_{0}, \mathcal{V}\right\rangle+\left\langle\mathcal{V}, q_{0}\right\rangle\right]\left[\overline{\left\langle q_{0}, \mathcal{V}\right\rangle}+\overline{\left\langle\mathcal{V}, q_{0}\right\rangle}\right]\left\langle q_{0}, q_{0}\right\rangle \\
& -\frac{\left(t_{s}(0)\right)^{3}}{\sqrt{\mathcal{F}(0)}} \frac{\left\langle q_{0}, \mathcal{V}\right\rangle+\left\langle\mathcal{V}, q_{0}\right\rangle}{2}\left\langle q_{0}, \mathcal{V}\right\rangle \\
& -\frac{\left(t_{s}(0)\right)^{3}}{\sqrt{\mathcal{F}(0)}} \frac{\overline{\left\langle q_{0}, \mathcal{V}\right\rangle}+\overline{\left\langle\mathcal{V}, q_{0}\right\rangle}}{2}\left\langle\mathcal{V}, q_{0}\right\rangle+\left(t_{s}(0)\right)^{2}\langle\mathcal{V}, \mathcal{V}\rangle
\end{aligned}
$$


Next we get

$$
\begin{aligned}
& \left\|\frac{\partial Z(\delta)}{\partial \delta}\right\|_{\delta=0}^{2} \\
& =\frac{\left(t_{s}(0)\right)^{4}}{4 \mathcal{F}(0)}\left[\left\langle q_{0}, \mathcal{V}\right\rangle+\left\langle\mathcal{V}, q_{0}\right\rangle\right]\left[\overline{\left\langle q_{0}, \mathcal{V}\right\rangle}+\overline{\left\langle\mathcal{V}, q_{0}\right\rangle}\right]\left(\left\|q_{0}\right\|^{2}+\gamma_{s} \bar{\gamma}_{s}\right) \\
& -\frac{\left(t_{s}(0)\right)^{3}}{\sqrt{\mathcal{F}(0)}} \frac{\overline{\left\langle q_{0}, \mathcal{V}\right\rangle}+\overline{\left\langle\mathcal{V}, q_{0}\right\rangle}}{2}\left\langle q_{0}, \mathcal{V}\right\rangle \\
& -\frac{\left(t_{s}(0)\right)^{3}}{\sqrt{\mathcal{F}(0)}} \frac{\overline{\left\langle q_{0}, \mathcal{V}\right\rangle}+\overline{\left\langle\mathcal{V}, q_{0}\right\rangle}}{2}\left\langle\mathcal{V}, q_{0}\right\rangle+\left(t_{s}(0)\right)^{2}\langle\mathcal{V}, \mathcal{V}\rangle \\
& =\frac{\left(t_{s}(0)\right)^{4}}{4 \mathcal{F}(0)}\left[\left\langle q_{0}, \mathcal{V}\right\rangle+\left\langle\mathcal{V}, q_{0}\right\rangle\right]\left[\left\langle q_{0}, \mathcal{V}\right\rangle+\left\langle\mathcal{V}, q_{0}\right\rangle\right]\left(\left\|q_{0}\right\|^{2}+\gamma_{s} \bar{\gamma}_{s}\right) \\
& -\frac{\left(t_{s}(0)\right)^{3}}{2 \sqrt{\mathcal{F}(0)}}\left[\left\langle q_{0}, \mathcal{V}\right\rangle+\left\langle\mathcal{V}, q_{0}\right\rangle\right]\left[\left\langle q_{0}, \mathcal{V}\right\rangle+\left\langle\mathcal{V}, q_{0}\right\rangle\right]+\left(t_{s}(0)\right)^{2}\langle\mathcal{V}, \mathcal{V}\rangle \\
& =\frac{\left(t_{s}(0)\right)^{4}}{4 \mathcal{F}(0)}\left[\left\langle q_{0}, \mathcal{V}\right\rangle+\left\langle\mathcal{V}, q_{0}\right\rangle\right]^{2}\left(\left\|q_{0}\right\|^{2}+\gamma_{s} \bar{\gamma}_{s}\right) \\
& -\frac{\left(t_{s}(0)\right)^{3}}{2 \sqrt{\mathcal{F}(0)}}\left[\left\langle q_{0}, \mathcal{V}\right\rangle+\left\langle\mathcal{V}, q_{0}\right\rangle\right]^{2}+\left(t_{s}(0)\right)^{2}\langle\mathcal{V}, \mathcal{V}\rangle
\end{aligned}
$$

Thus we have

$$
\begin{aligned}
& \left\|\frac{\partial Z(\delta)}{\partial \delta}\right\|_{\delta=0}^{2}= \\
& \frac{\left(t_{s}(0)\right)^{2}}{2 \sqrt{\mathcal{F}(0)}}\left[\left\langle q_{0}, \mathcal{V}\right\rangle+\left\langle\mathcal{V}, q_{0}\right\rangle\right]^{2}\left(\frac{\left(t_{s}(0)\right)^{2}}{2 \sqrt{\mathcal{F}(0)}}\left(\left\|q_{0}\right\|^{2}+\gamma_{s} \bar{\gamma}_{s}\right)-t_{s}(0)\right) \\
& +\left(t_{s}(0)\right)^{2}\langle\mathcal{V}, \mathcal{V}\rangle .
\end{aligned}
$$

Substituting the expression given in equation (7.1.34) into (7.1.37) yields

$$
\begin{aligned}
& \left\|\frac{\partial Z(\delta)}{\partial \delta}\right\|_{\delta=0}^{2} \\
& =\frac{\left(t_{s}(0)\right)^{2}}{2 \sqrt{\mathcal{F}(0)}}\left[\left\langle q_{0}, \mathcal{V}\right\rangle+\left\langle\mathcal{V}, q_{0}\right\rangle\right]^{2} \frac{\alpha}{2 \sqrt{\mathcal{F}(0)}}+\left(t_{s}(0)\right)^{2} \\
& =\frac{\alpha\left(t_{s}(0)\right)^{2}}{4 \mathcal{F}(0)}\left[\left\langle q_{0}, \mathcal{V}\right\rangle+\left\langle\mathcal{V}, q_{0}\right\rangle\right]^{2}+\left(t_{s}(0)\right)^{2}
\end{aligned}
$$


Similarly,

$$
\begin{aligned}
& \left\|\frac{\partial \hat{Z}(\delta)}{\partial \delta}\right\|_{\delta=0}^{2} \\
& =\frac{\alpha\left(t_{s}(0)\right)^{2}}{4 \mathcal{F}(0)}\left[\left\langle q_{0}, \hat{\mathcal{V}}\right\rangle+\left\langle\hat{\mathcal{V}}, q_{0}\right\rangle\right]^{2}+\left(t_{s}(0)\right)^{2}
\end{aligned}
$$

Next we calculate the product

$$
\left\|\frac{\partial Z(\delta)}{\partial \delta}\right\|_{\delta=0}^{2} \cdot\left\|\frac{\partial \hat{Z}(\delta)}{\partial \delta}\right\|_{\delta=0}^{2} .
$$

We have

$$
\begin{aligned}
& \left\|\frac{\partial Z(\delta)}{\partial \delta}\right\|_{\delta=0}^{2} .\left\|\frac{\partial \hat{Z}(\delta)}{\partial \delta}\right\|_{\delta=0}^{2} \\
& =\left[\left(t_{s}(0)\right)^{2}+\frac{\alpha\left(t_{s}(0)\right)^{2}}{4 \mathcal{F}(0)}\left[\left\langle q_{0}, \mathcal{V}\right\rangle+\left\langle\mathcal{V}, q_{0}\right\rangle\right]^{2}\right] \\
& =\left[\left(t_{s}(0)\right)^{2}+\frac{\alpha\left(t_{s}(0)\right)^{2}}{4 \mathcal{F}(0)}\left[\left\langle q_{0}, \hat{\mathcal{V}}\right\rangle+\left\langle\hat{\mathcal{V}}, q_{0}\right\rangle\right]^{2}\right] \\
& +\frac{\alpha\left(t_{s}(0)\right)^{4}}{4 \mathcal{F}(0)}\left[\left\langle q_{0}, \mathcal{V}\right\rangle+\left\langle\mathcal{V}, q_{0}\right\rangle\right]^{2} \\
& \left.\left.+\left(\frac{\alpha\left(t_{s}(0)\right)^{4}}{4 \mathcal{F}(0))^{2}}\right)^{2}\left[\left\langle q_{0}, \mathcal{V}\right\rangle+\langle\mathcal{V}\rangle+\left\langle\hat{\mathcal{V}}, q_{0}\right\rangle\right]^{2}\right\rangle\right]^{2}\left[\left\langle q_{0}, \hat{\mathcal{V}}\right\rangle+\left\langle\hat{\mathcal{V}}, q_{0}\right\rangle\right]^{2}
\end{aligned}
$$

Thus

$$
\begin{aligned}
& \left\|\frac{\partial Z(\delta)}{\partial \delta}\right\|_{\delta=0}^{2} \cdot\left\|\frac{\partial \hat{Z}(\delta)}{\partial \delta}\right\|_{\delta=0}^{2} \\
& =\left(t_{s}(0)\right)^{4}+\frac{\alpha\left(t_{s}(0)\right)^{4}}{4 \mathcal{F}(0)}\left(\left[\left\langle q_{0}, \hat{\mathcal{V}}\right\rangle+\left\langle\hat{\mathcal{V}}, q_{0}\right\rangle\right]^{2}+\left[\left\langle q_{0}, \mathcal{V}\right\rangle+\left\langle\mathcal{V}, q_{0}\right\rangle\right]^{2}\right) \\
& +\left(\frac{\alpha\left(t_{s}(0)\right)^{2}}{4 \mathcal{F}(0)}\right)^{2}\left[\left\langle q_{0}, \mathcal{V}\right\rangle+\left\langle\mathcal{V}, q_{0}\right\rangle\right]^{2}\left[\left\langle q_{0}, \hat{\mathcal{V}}\right\rangle+\left\langle\hat{\mathcal{V}}, q_{0}\right\rangle\right]^{2}
\end{aligned}
$$


Factoring $\left(t_{s}(0)\right)^{4}$ from equation (7.1.41) and substituting $\mathcal{J}$ and $\Lambda$ expressed in equations (7.1.10) and (7.1.11), respectively, we see that

$$
\left\|\frac{\partial Z(\delta)}{\partial \delta}\right\|_{\delta=0}^{2} .\left\|\frac{\partial \hat{Z}(\delta)}{\partial \delta}\right\|_{\delta=0}^{2}=\left(t_{s}(0)\right)^{4}\left(1+\mathcal{J}^{2}+\Lambda\right)
$$

Recall that $\cos (\mathcal{Y})$ is the quotient of

$\left.\left\langle\frac{\partial Z(\delta)}{\partial \delta}, \frac{\partial \hat{Z}(\delta)}{\partial \delta}\right\rangle_{H_{N}}\right|_{\delta=0}+\left.\left\langle\frac{\partial \hat{Z}(\delta)}{\partial \delta}, \frac{\partial Z(\delta)}{\partial \delta}\right\rangle_{H_{N}}\right|_{\delta=0}$ and $2\left\|\frac{\partial Z(\delta)}{\partial \delta}\right\|_{\delta=0} \cdot\left\|\frac{\partial \hat{Z}(\delta)}{\partial \delta}\right\|_{\delta=0}$. Therefore, the division of (7.1.36) by 2 times the square root of the right hand side of equation (7.1.42) yields the desired formula (7.1.9).

\subsection{Similarity of Triangles}

Prior to stating the main result of this section we note the following. Let $Q=(\overrightarrow{0}, q) \in \mathbb{S}_{N}$ and let $\hat{Q}=(\overrightarrow{0}, \hat{q}) \in \mathbb{S}_{n}$ be another arbitrary point in $\mathbb{S}_{N}$ and let $Z, \hat{Z}$ be the projected points of $Q, \hat{Q}$ on the sphere respectively, such that in analogy to $((3.5 .11))$ and $((3.5 .10))$ we have

$$
\operatorname{SPM}\left(\theta_{s}, R, \gamma_{s}\right)[(0, \hat{q})]=\left(\left(1-\hat{t}_{s}\right) \gamma_{s} u, \hat{t}_{s} \hat{q}\right)
$$

with

$$
\hat{t}_{s}=\frac{-\frac{\mathcal{A}}{2}+\sqrt{\hat{F}}}{\|\hat{q}\|^{2}+\gamma_{s} \bar{\gamma}_{s}}, \hat{F}:=\frac{\mathcal{A}^{2}}{4}-\left(\|\hat{q}\|^{2}+\gamma_{s} \bar{\gamma}_{s}\right) \alpha .
$$

We are now ready to prove

Lemma 7.2.1 i) $\forall Q \in S_{N}, \forall \hat{Q} \in S_{N}, P Q \hat{Q} \approx P \hat{Z} Z \Longleftrightarrow$

$$
I:=-\alpha\left(\|\hat{q}\|^{2}-\|q\|^{2}\right)\left[1+\frac{-\mathcal{A}}{\sqrt{F}+\sqrt{\hat{F}}}\right]=0 .
$$

ii) Moreover, if $\alpha=0$ then $I=0 \forall Q \in \mathbb{S}_{N}, \forall \hat{Q} \in S_{N}$ so $P Q \hat{Q} \approx P \hat{Z} Z$. If $\alpha<0$ then $P Q \hat{Q} \approx P \hat{Z} Z$ iff

$$
\|\hat{q}\|=\|q\| \text {. }
$$

Proof We observe that $P Q \hat{Q} \approx P \hat{Z} Z$ iff

$$
\frac{\|\overrightarrow{P Z}\|_{H_{N}}^{2}}{\|\overrightarrow{P Q}\|_{H_{N}}^{2}}=\frac{\|\overrightarrow{P Z}\|_{H_{N}}^{2}}{\|\overrightarrow{P Q}\|_{H_{N}}^{2}} \Longleftrightarrow
$$




$$
I=\|\overrightarrow{P Z}\|_{H_{N}}^{2}\|\overrightarrow{P Q}\|_{H_{N}}^{2}-\|\overrightarrow{P Z}\|_{H_{N}}^{2}\|\overrightarrow{P Q}\|_{H_{N}}^{2}=0 .
$$

We now proceed to calculate $I$ in $((7.2 .5))$ by calculating separately each term in $((7.2 .5))$ as follows. We have

$$
\begin{gathered}
\|\overrightarrow{P Q}\|_{H_{N}}^{2}=\langle Q-P, Q-P\rangle_{H_{N}}=\|q\|^{2}+\gamma_{s} \bar{\gamma}_{s} . \\
\|\overrightarrow{P Q}\|_{H_{N}}^{2}=\langle\hat{Q}-P,-\hat{Q} P\rangle_{H_{N}}=\|\hat{q}\|^{2}+\gamma_{s} \bar{\gamma}_{s} \\
\|\overrightarrow{P Z}\|_{H_{N}}^{2}=\langle Z-P, Z-P\rangle_{H_{N}}=\|z\|^{2}+\left(c-\gamma_{s}\right) \overline{\left(c-\gamma_{s}\right)} \\
\|\overrightarrow{P Z}\|_{H_{N}}^{2}=\langle\hat{Z}-P, \hat{Z}-P\rangle_{H_{N}}=\|\hat{z}\|^{2}+\left(\hat{c}-\gamma_{s}\right) \overline{\left(\hat{c}-\gamma_{s}\right)}
\end{gathered}
$$

since $c-\gamma_{s}=\left(1-t_{s}\right) \gamma_{s}-\gamma_{s}=-t_{s} \gamma_{s}$ and since $\hat{c}-\gamma_{s}=-\hat{t}_{s} \gamma_{s}$ then

$$
\|\overrightarrow{P Z}\|_{H_{N}}^{2}=t_{s}^{2}\left(\|q\|^{2}+\gamma_{s} \bar{\gamma}_{s}\right), \quad\|\overrightarrow{P \hat{Z}}\|_{H_{N}}^{2}=\hat{t}_{s}^{2}\left(\|\hat{q}\|^{2}+\gamma_{s} \bar{\gamma}_{s}\right) .
$$

Substituting the expressions for $\|\overrightarrow{P Q}\|_{H_{N}}^{2},\|\overrightarrow{P Q}\|_{H_{N}}^{2},\|\overrightarrow{P Z}\|_{H_{N}}^{2}$ and $\|\overrightarrow{P Z}\|_{H_{N}}^{2}$ into the expression for $I$ defined by $((7.2 .5))$

we obtain

$$
\begin{aligned}
I & =\left[\hat{t}_{s}^{2}\|\hat{q}\|^{2}+\hat{t}_{s}^{2} \gamma_{s} \bar{\gamma}_{s}\right]\left[\|\hat{q}\|^{2}+\gamma_{s} \bar{\gamma}_{s}\right]-\left[t_{s}^{2}\|q\|^{2}+t_{s}^{2} \gamma_{s} \bar{\gamma}_{s}\right]\left[\|q\|^{2}+\gamma_{s} \bar{\gamma}_{s}\right] \\
& =\hat{t}_{s}^{2}\left[\|\hat{q}\|^{2}+\gamma_{s} \bar{\gamma}_{s}\right]^{2}-t_{s}^{2}\left[\|q\|^{2}+\gamma_{s} \bar{\gamma}_{s}\right]^{2} \\
& =\left(-\frac{\mathcal{A}}{2}+\sqrt{\hat{F}}\right)^{2}-\left(-\frac{\mathcal{A}}{2}+\sqrt{F}\right)^{2} \\
& =\left[\left(-\frac{\mathcal{A}}{2}+\sqrt{\hat{F}}\right)-\left(-\frac{\mathcal{A}}{2}+\sqrt{F}\right)\right]\left[\left(-\frac{\mathcal{A}}{2}+\sqrt{\hat{F}}\right)+\left(-\frac{\mathcal{A}}{2}+\sqrt{F}\right)\right] \\
& =[\sqrt{\hat{F}}-\sqrt{F}][-\mathcal{A}+\sqrt{F}+\sqrt{\hat{F}}]=\frac{\hat{F}-F}{\sqrt{F}+\sqrt{\hat{F}}}[-\mathcal{A}+\sqrt{F}+\sqrt{\hat{F}}] \\
& =(\hat{F}-F)\left[1+\frac{-\mathcal{A}}{\sqrt{F}+\sqrt{\hat{F}}}\right] .
\end{aligned}
$$

Substitute in the above the relation $(\hat{F}-F)=-\alpha\left(\|\hat{q}\|^{2}-\|q\|^{2}\right)$ to obtain $((7.2 .3))$. In order to prove ii) notice that

$$
\frac{-\mathcal{A}}{\sqrt{F}+\sqrt{\hat{F}}} \geq 0 \Longrightarrow\left[1+\frac{-\mathcal{A}}{\sqrt{F}+\sqrt{\hat{F}}}\right] \geq 1,
$$


and the conclusions follow.

Remark The points $P, Q, \hat{Q}, Z, \hat{Z}$ all lie in one plane. The points $Q, \hat{Q}, Z, \hat{Z}$ lie on one circle with radius $\sqrt{\|\overrightarrow{P Z}\|_{H_{N}}\|\overrightarrow{P Q}\|_{H_{N}}}$ and the quantity $\|\overrightarrow{P Z}\|_{H_{N}}\|\overrightarrow{P Q}\|_{H_{N}}$ may be defined in analogy to Euclidean geometry as the power $P O W E R$ of the points $Q, Z$, with respect to the point $P$ so that $P O W E R(Q, Z, P):=$ $\|\overrightarrow{P Z}\|_{H_{N}}\|\overrightarrow{P Q}\|_{H_{N}}$. Recall from $((7.2 .6))$ and $((7.2 .7))$ that we have in a complex Hilbert space

$$
\operatorname{POWER}(Q, Z, P)=t_{s}\left[\|q\|^{2}+\gamma_{s} \bar{\gamma}_{s}\right]=\left[-\frac{\mathcal{A}}{2}+\sqrt{\frac{\mathcal{A}^{2}}{4}-\left(\|q\|^{2}+\gamma_{s} \bar{\gamma}_{s}\right) \alpha}\right]
$$

Thus we have proven

Proposition 7.2.2 The POWER(Q,Z,P) of the points $Q, Z$ under a projection is a sole functions of $\|q\|$. It is a constant independent of $\|q\|$ iff the pojection is the stereographic one.

\subsection{Reformulation of The Main Theorem and Its Proof}

We are ready to reformulate the main theorem of this chapter that is announced in the introduction section and to prove it utilizing the notations and the lemmas of the previous sections.

Theorem 7.3.1 The following conditions are equivalent. i) $\alpha=0$. ii) $\forall \mathcal{X}, \mathcal{Y}=$ $\mathcal{X}$. iii) $\forall Q \forall \hat{Q}, P Q \hat{Q} \approx P \hat{Z} Z$.

Let $\alpha=0$. Then by (7.1.9) we have for all choices of sphere parameters $0 \neq \gamma_{s}, \theta_{s}, R$ subject to assumptions III) and IV) and for any $\cos (\mathcal{X}), \cos (\eta), \cos (\hat{\eta})$ and $\left\|q_{0}\right\|$ we have $\mathcal{J}=\Lambda=0$ and by (7.1.9) we have $\cos (\mathcal{Y})=\cos (\mathcal{X}) \Longrightarrow \mathcal{Y}=\mathcal{X}$.

It remains to show that if $\alpha \neq 0$ there exist angles $\mathcal{X}$ such that $\cos (\mathcal{Y}) \neq$ $\cos (\mathcal{X})$. We prove this by contradiction. Recall (7.1.2) and fix $U_{0}:=\left\|q_{0}\right\|^{-1} q_{0}$ but allow $\left\|q_{0}\right\|>0$ to be a free variable and let

$$
\cos (\eta)=\frac{\left\langle U_{0}, \mathcal{V}\right\rangle+\left\langle\mathcal{V}, U_{0}\right\rangle}{2}, \cos (\hat{\eta})=\frac{\left\langle U_{0}, \hat{\mathcal{V}}\right\rangle+\left\langle\hat{\mathcal{V}}, U_{0}\right\rangle}{2}
$$


Now choose $\mathcal{V}, \hat{\mathcal{V}}$ such that $-\frac{1}{4} \leq \cos (\mathcal{X})=\frac{\langle\mathcal{V}, \hat{\mathcal{V}}\rangle+\langle\hat{\mathcal{V}}, \mathcal{V}\rangle}{2} \leq \frac{1}{4}$. Next choose $U_{0}$ such that $\cos (\eta) \cos (\hat{\eta}) \neq 0$ and observe that thanks to $\alpha<0$ that

$$
\begin{gathered}
\lim _{\left\|q_{0}\right\| \rightarrow \infty}|\mathcal{J}|=\infty \Longrightarrow \lim _{\left\|q_{0}\right\| \rightarrow \infty} \frac{\Lambda}{\left|\mathcal{J}^{2}\right|}=0 \Longrightarrow \\
\lim _{\left\|q_{0}\right\| \rightarrow \infty} \frac{\mathcal{J}}{\left.\sqrt{1+\mathcal{J}^{2}+\Lambda}-1\right\}}=\lim _{\left\|q_{0}\right\| \rightarrow \infty} \frac{\mathcal{J}}{|\mathcal{J}|}=\operatorname{sgn}(\alpha \cos (\eta) \cos (\hat{\eta})) .
\end{gathered}
$$

Notice that if $\forall \mathcal{X}, \mathcal{Y}=\mathcal{X} \Longrightarrow \cos (\mathcal{Y})=\cos (\mathcal{X})$ and then by (7.1.9) we have

$$
\sqrt{1+\mathcal{J}^{2}+\Lambda} \cos (\mathcal{X})=\cos (\mathcal{X})+\mathcal{J} \Longrightarrow \cos (\mathcal{X})=\frac{\mathcal{J}}{\sqrt{1+\mathcal{J}^{2}+\Lambda}-1},
$$

Now let $\left\|q_{0}\right\|$ to be large enough to make $|\cos (\mathcal{X})|=\left|\frac{\mathcal{J}}{\sqrt{1+\mathcal{J}^{2}+\Lambda}-1}\right|>\frac{1}{4}$ and the contradiction follows.

It is noteworthy that even if $\alpha \neq 0$, certain special angles $\mathcal{X}$ are preserved under all spherical boundizations. Most conspicuous are all the angles with vertex at the origin, namely those where $q_{0}=0$. Then, it is easily observed that $\mathcal{J}=\Lambda=0$ and by (7.1.9) we have $\cos (\mathcal{Y})=\cos (\mathcal{X}) \Longrightarrow \mathcal{Y}=\mathcal{X}$. Next we observe that with $\left\|q_{0}\right\| \neq 0$ all the angles with $\mathcal{V}, \hat{\mathcal{V}}$ such that

$$
\left\langle q_{0}, \mathcal{V}\right\rangle=\left\langle\mathcal{V}, q_{0}\right\rangle=\left\langle q_{0}, \hat{\mathcal{V}}\right\rangle=\left\langle\hat{\mathcal{V}}, q_{0}\right\rangle=0
$$

we have again $\mathcal{J}=\Lambda=0 \Longrightarrow \mathcal{Y}=\mathcal{X}$.

\subsection{Examples and Utility of The Multi Pa- rameter Family}

The advantages of having a multi-parameter family of projections rather than one "canonical" form are several. It provides an efficient unified framework. If $\theta_{s}=0$ and $\gamma_{s}=1=R$, we obtain the stereographic projection for $H=\mathbb{R}^{2}$ employed in L. V. Ahlfors [2] (chapter one, pages 19-20). If $\theta_{s}=\frac{1}{2}=R$ and $\gamma_{s}=1$ we obtain for $H=\mathbb{R}^{2}$ the stereographic projection obtained in E. Hille [31] (part one, chapter two, pages 42-43). If $\gamma_{s}=\theta_{s}=\frac{1}{2}=R$ then we obtain 
for $\mathbb{H}=\mathbb{R}^{2}$ the Poincare "compactification". All three derivations follow from the one set of formulas given in here. Notice that in both derivations of the stereographic projections we compute and find $\alpha=0$ which is consistent with our generalized definition of a stereographic projection in a complex Hilbert space.

It goes without saying that a multi parameter family of projections provides mathematical means to view objects in different perspectives. It is noteworthy though that if the projection point $\left(\gamma_{s} u, 0\right)$ is chosen to coincide with the coordinate center $\left(\gamma_{s} u, 0\right)=(0,0)$, then by II) we also have then that $\theta_{s}=0$, and a degeneracy and catastrophe besets the nonlinear projection.

Degeneracy of a nonlinear projection.

The relevant quantities become

$$
\begin{aligned}
& \mathcal{A}=0, \alpha=-R^{2}, F=R^{2}\|q\|^{2}, t_{s}=\frac{R}{\|q\|}, \\
& z=t_{s} q=\frac{R}{\|q\|} q \text { and } c u=0 \text { if }\|q\| \neq 0 .
\end{aligned}
$$

It is readily observed that $t_{s}$ becomes an unbounded quantity as $\|q\| \rightarrow 0$ and $z$ becomes a discontinuous mapping as $\|q\| \rightarrow 0$. Moreover, all the points $(\overrightarrow{0}, q) \in \mathbb{H}_{N}, q \in \mathbb{H}$ that lie on the fixed "ray" $U=\frac{1}{\|q\|} q=$ constant, map onto one point $(\overrightarrow{0, z}) \in \mathbb{H}_{N}, z=R U \in \mathbb{H}$. This mathematical aspect could serve as a guidance to those who need to locate a spherical lens in a proper location to obtain low distortion. Images are going to be highly distorted if the projection point is placed too close to the projected plane $\mathbb{R}^{2}$.

The several parameters on which the family of boundizations depends satisfies additional purposes. It brings out the fact that the celebrated stereographic projection is a degenerate member of a family of non linear projections that preserve all directions at infinity. This can easily be seen in $\mathbb{R}^{3}$ in [26]. For $\alpha=0$ all directions arrows $\infty(\cos (\theta), \sin (\theta))$ in $\mathbb{R}^{2}$ are mapped on the north pole (or the south pole) and shrink to a point. This is in contrast to the case $\alpha<0$ where all directions $\infty(\cos (\theta), \sin (\theta))$ in $\mathbb{R}^{2}$ are preserved and $\mathbb{R}^{2}$ is mapped onto a subset of a sphere that is "bowl shaped". 
The rich family of boundizations shows also the special place that Poincare's compactification fits within our larger family.

In the following examples we compute the measure of angles between vectors in various specialized Hilbert spaces and the measure of their images for the purpose of making the scope of this chapter more tangible. Recall that $\mathcal{A}=-2 \gamma_{s} \bar{\gamma}_{s}+\gamma_{s} \bar{\theta}_{s}+\theta_{s} \bar{\gamma}_{s}, \alpha=\left|\gamma_{s}-\theta_{s}\right|^{2}-R^{2}$ and that

$$
\cos (\mathcal{X})=\frac{\langle\mathcal{V}, \hat{\mathcal{V}}\rangle+\langle\hat{\mathcal{V}}, \mathcal{V}\rangle}{2}, \cos (\mathcal{Y})=\frac{\cos (\mathcal{X})+\mathcal{J}}{\sqrt{1+\mathcal{J}^{2}+\Lambda}} .
$$

Example The space of all continuous complex-valued functions on $[a, b]$ with inner product defined by $\langle f, g\rangle=\int_{a}^{b} f(s) \bar{g}(s) d s$ where $f, g \in C[a, b]$ and $a, b$ are real numbers. Assume

$$
\left.\left[\int_{a}^{b} f(s) \bar{f}(s) d s\right]>0 \int_{a}^{b} g(s) \bar{g}(s) d s\right]^{-\frac{1}{2}}>0 .
$$

Then,

$$
\begin{gathered}
\left.\mathcal{V}=\left[\int_{a}^{b} f(s) \bar{f}(s) d s\right]^{-\frac{1}{2}} f(s), \hat{\mathcal{V}}=\int_{a}^{b} g(s) \bar{g}(s) d s\right]^{-\frac{1}{2}} g(s), \\
\|\mathcal{V}\|^{2}=1, \quad\|\hat{\mathcal{V}}\|^{2}=1 .
\end{gathered}
$$

and

$$
\begin{aligned}
\mathcal{F}(0) & =\frac{\mathcal{A}^{2}}{4}-\alpha\left(\left\|q_{0}\right\|^{2}+\gamma_{s} \bar{\gamma}_{s}\right) \\
& =\frac{\mathcal{A}^{2}}{4}-\alpha\left[\int_{a}^{b} q_{0}(s) \bar{q}_{0}(s) d s+\gamma_{s} \bar{\gamma}_{s}\right]=\hat{\mathcal{F}}(0)
\end{aligned}
$$

Also we have

$$
\begin{aligned}
& \mathcal{J}=\frac{\alpha}{4 \mathcal{F}(0)}\{ \\
& \left.\left[\int_{a}^{b} q_{0}(s) \overline{\mathcal{V}}(s) d s+\int_{a}^{b} \mathcal{V}(s) \bar{q}_{0}(s) d s\right]\left[\int_{a}^{b} q_{0}(s) \overline{\hat{\mathcal{V}}}(s) d s+\int_{a}^{b} \hat{\mathcal{V}}(s) \bar{q}_{0}(s) d s\right]\right\}
\end{aligned}
$$

and 


$$
\begin{aligned}
& \Lambda=\frac{\alpha}{4 \mathcal{F}(0)}\left\{\left[\int_{a}^{b} q_{0}(s) \overline{\mathcal{V}}(s) d s+\int_{a}^{b} \mathcal{V}(s) \bar{q}_{0}(s) d s\right]^{2}\right. \\
& \left.+\left[\int_{a}^{b} q_{0}(s) \overline{\hat{\mathcal{V}}}(s) d s+\int_{a}^{b} \hat{\mathcal{V}}(s) \bar{q}_{0}(s) d s\right]^{2}\right\}
\end{aligned}
$$

Thus,

$$
\begin{aligned}
\cos (\mathcal{X}) & =\frac{\langle\mathcal{V}, \hat{\mathcal{V}}\rangle+\langle\hat{\mathcal{V}}, \mathcal{V}\rangle}{2}=\operatorname{Re}\langle\mathcal{V}, \hat{\mathcal{V}}\rangle=\operatorname{Re} \int_{a}^{b} \mathcal{V}(s) \overline{\hat{\mathcal{V}}}(s) d s \\
& =\left[\int_{a}^{b} f(s) \bar{f}(s) d s\right]^{-\frac{1}{2}}\left[\int_{a}^{b} g(s) \bar{g}(s) d s\right]^{-\frac{1}{2}} \operatorname{Re} \int_{a}^{b} f(s) \bar{g}(s) d s
\end{aligned}
$$

and

$$
\cos (\mathcal{Y})=\frac{\left[\int_{a}^{b} f(s) \bar{f}(s) d s\right]^{-\frac{1}{2}}\left[\int_{a}^{b} g(s) \bar{g}(s) d s\right]^{-\frac{1}{2}} R e \int_{a}^{b} f(s) \bar{g}(s) d s+\mathcal{J}}{\sqrt{1+\mathcal{J}^{2}+\Lambda}}
$$

with $\mathcal{J}$ and $\Lambda$ subject to (7.4.3) and (7.4.4) respectively.

Next we have

Example The space of all square matrices $M^{n x n} A, B \in M^{n x n}$ with inner product defined by $\left\langle A, B>:=\operatorname{Trace}\left(A^{*} B\right)\right.$. Assume $\operatorname{Trace}\left(A^{*} A\right)>0$ and $\operatorname{Trace}\left(B^{*} B\right)>0$ then we have $\mathcal{V}=\left(\operatorname{Trace}\left(A^{*} A\right)\right)^{-\frac{1}{2}} A$, and $\hat{\mathcal{V}}=$ $\left(\operatorname{Trace}\left(B^{*} B\right)\right)^{-\frac{1}{2}} B,\|\mathcal{V}\|^{2}=\|\hat{\mathcal{V}}\|^{2}=1$. and

$$
\mathcal{F}(0)=\frac{\mathcal{A}^{2}}{4}-\alpha\left(\left\|q_{0}\right\|^{2}+\gamma_{s} \bar{\gamma}_{s}\right)=\frac{\mathcal{A}^{2}}{4}-\alpha\left[\operatorname{Trace}\left(q_{0}^{*} q_{0}\right)+\gamma_{s} \bar{\gamma}_{s}\right]=\hat{\mathcal{F}}(0)
$$

Also we have

$$
\mathcal{J}=\frac{\alpha}{4 \mathcal{F}(0)}\left[\operatorname{Trace}\left(q_{0}^{*} \mathcal{V}\right)+\operatorname{Trace}\left(\mathcal{V}^{*} q_{0}\right)\right]\left[\operatorname{Trace}\left(q_{0}^{*} \hat{\mathcal{V}}\right)+\operatorname{Trace}\left(\hat{\mathcal{V}}^{*} q_{0}\right)\right]
$$

and

$$
\Lambda=\frac{\alpha}{4 \mathcal{F}(0)}\left\{\left[\operatorname{Trace}\left(q_{0}^{*} \hat{\mathcal{V}}\right)+\operatorname{Trace}\left(\hat{\mathcal{V}}^{*} q_{0}\right)\right]^{2}+\left[\operatorname{Trace}\left(q_{0}^{*} \mathcal{V}\right)+\operatorname{Trace}\left(\mathcal{V}^{*} q_{0}\right)\right]^{2}\right\}
$$


Thus,

$$
\cos (\mathcal{X})=\left(\operatorname{Trace}\left(A^{*} A\right)\right)^{-\frac{1}{2}}\left(\operatorname{Trace}\left(B^{*} B\right)\right)^{-\frac{1}{2}} \operatorname{Trace}\left(A^{*} B\right)
$$

and

$$
\cos (\mathcal{Y})=\frac{\left(\operatorname{Trace}\left(A^{*} A\right)\right)^{-\frac{1}{2}}\left(\operatorname{Trace}\left(B^{*} B\right)\right)^{-\frac{1}{2}} \operatorname{Trace}\left(A^{*} B\right)+\mathcal{J}}{\sqrt{1+\mathcal{J}^{2}+\Lambda}}
$$

with $\mathcal{J}$ and $\Lambda$ subject to (7.4.5) and (7.4.6) respectively .

Next we have

Example Take $\mathbb{H}=\mathbb{C}^{n}$ as a Hilbert space over $\mathbb{C}$ and define

$$
\langle q, \hat{q}\rangle=x_{1} \overline{\hat{x}}_{1}+\cdots+x_{n} \overline{\hat{x}}_{n}
$$

where $q=\left(x_{1}, \ldots, x_{n}\right)$ and $\hat{q}=\left(\hat{x}_{1}, \ldots \hat{x}_{n}\right)$. Assume $q_{0}=\left(x_{0_{1}}, \ldots, x_{0_{n}}\right)$, $\left(x_{1} \bar{x}_{1}+\cdots+x_{n} \bar{x}_{n}\right)>0$ and $\left(\hat{x}_{1} \overline{\hat{x}}_{1}+\cdots+\hat{x}_{n} \overline{\hat{x}}_{n}\right)>0$, then we have $\mathcal{V}=$ $\left(x_{1} \bar{x}_{1}+\cdots+x_{n} \bar{x}_{n}\right)^{-\frac{1}{2}}\left(x_{1} \ldots x_{n}\right)$ and $\hat{\mathcal{V}}=\left(\hat{x}_{1} \overline{\hat{x}}_{1}+\cdots+\hat{x}_{n} \overline{\hat{x}}_{n}\right)^{-\frac{1}{2}}\left(\hat{x}_{1} \ldots \hat{x}_{n}\right)$, $\|\mathcal{V}\|^{2}=\|\hat{\mathcal{V}}\|^{2}=1$. Thus we have $\mathcal{A}=-2 \gamma_{s} \bar{\gamma}_{s}+\gamma_{s} \bar{\theta}_{s}+\theta_{s} \bar{\gamma}_{s}, \alpha=\gamma_{s} \bar{\gamma}_{s}-$ $\gamma_{s} \bar{\theta}_{s}-\theta_{s} \bar{\gamma}_{s}+\theta_{s} \bar{\theta}_{s}-R^{2}$ and

$$
\begin{aligned}
\mathcal{F}(0) & =\frac{\mathcal{A}^{2}}{4}-\alpha\left(\left\|q_{0}\right\|^{2}+\gamma_{s} \bar{\gamma}_{s}\right) \\
& =\frac{\mathcal{A}^{2}}{4}-\alpha\left[\left(x_{0_{1}} \bar{x}_{0_{1}}+\cdots+x_{0_{n}} \bar{x}_{0_{n}}\right)+\gamma_{s} \bar{\gamma}_{s}\right]=\hat{\mathcal{F}}(0)
\end{aligned}
$$

Also we have

$$
\mathcal{J}=\frac{\alpha}{4 \mathcal{F}(0)}\left[\left\langle q_{0}, \mathcal{V}\right\rangle+\left\langle\mathcal{V}, q_{0}\right\rangle\right]\left[\left\langle q_{0}, \hat{\mathcal{V}}\right\rangle+\left\langle\hat{\mathcal{V}}, q_{0}\right\rangle\right]
$$

and

$$
\Lambda=\frac{\alpha}{4 \mathcal{F}(0)}\left(\left[\left\langle q_{0}, \hat{\mathcal{V}}\right\rangle+\left\langle\hat{\mathcal{V}}, q_{0}\right\rangle\right]^{2}+\left[\left\langle q_{0}, \mathcal{V}\right\rangle+\left\langle\mathcal{V}, q_{0}\right\rangle\right]^{2}\right)
$$

where

$$
\begin{aligned}
& \left\langle q_{0}, \mathcal{V}\right\rangle+\left\langle\mathcal{V}, q_{0}\right\rangle=\left(x_{0_{1}} \bar{v}_{1}+\cdots+x_{0_{n}} \bar{v}_{n}\right)+\left(v_{1} \bar{x}_{0_{1}}+\cdots+v_{n} \bar{x}_{0_{n}}\right) \\
& \left\langle q_{0}, \hat{\mathcal{V}}\right\rangle+\left\langle\hat{\mathcal{V}}, q_{0}\right\rangle=\left(x_{0_{1}} \overline{\hat{v}}_{1}+\cdots+x_{0_{n}} \overline{\hat{v}}_{n}\right)+\left(\hat{v}_{1} \bar{x}_{0_{1}}+\cdots+\hat{v}_{n} \bar{x}_{0_{n}}\right)
\end{aligned}
$$


Thus,

$$
\cos (\mathcal{X})=\left(x_{1} \bar{x}_{1}+\cdots+x_{n} \bar{x}_{n}\right)^{-\frac{1}{2}}\left(\hat{x}_{1} \overline{\hat{x}}_{1}+\cdots+\hat{x}_{n} \overline{\hat{x}}_{n}\right)^{-\frac{1}{2}}\left(x_{1} \overline{\hat{x}}_{1}+\cdots+x_{n} \overline{\hat{x}}_{n}\right)
$$

and

$$
\begin{aligned}
& \cos (\mathcal{Y}) \\
& =\frac{\left(x_{1} \bar{x}_{1}+\cdots+x_{n} \bar{x}_{n}\right)^{-\frac{1}{2}}\left(\hat{x}_{1} \overline{\hat{x}}_{1}+\cdots+\hat{x}_{n} \overline{\hat{x}}_{n}\right)^{-\frac{1}{2}}\left(x_{1} \overline{\hat{x}}_{1}+\cdots+x_{n} \overline{\hat{x}}_{n}\right)+\mathcal{J}}{\sqrt{1+\mathcal{J}^{2}+\Lambda}}
\end{aligned}
$$

with $\mathcal{J}$ and $\Lambda$ subject to (7.4.7) and (7.4.8) respectively.

Next we have

Example Take $\mathbb{H}=\mathbb{C}^{n}$ as a Hilbert space over $\mathbb{R}$ and define

$$
\langle q, \hat{q}\rangle=x_{1} \overline{\hat{x}}_{1}+\cdots+x_{n} \overline{\hat{x}}_{n}
$$

where $q=\left(x_{1}, \ldots, x_{n}\right)$ and $\hat{q}=\left(\hat{x}_{1}, \ldots \hat{x}_{n}\right)$.

Assume $q_{0}=\left(x_{0_{1}}, \ldots, x_{0_{n}}\right),\left(x_{1} \bar{x}_{1}, \ldots, x_{n} \bar{x}_{n}\right)>0$ and $\left(\hat{x}_{1} \overline{\hat{x}}_{1}, \ldots \hat{x}_{n} \overline{\hat{x}}_{n}\right)>0$ $\mathcal{V}=\left(x_{1} \bar{x}_{1}, \ldots, x_{n} \bar{x}_{n}\right)^{-\frac{1}{2}}\left(x_{1}, \ldots, x_{n}\right)$ and $\hat{\mathcal{V}}=\left(\hat{x}_{1} \overline{\hat{x}}_{1}, \ldots \hat{x}_{n} \overline{\hat{x}}_{n}\right)^{-\frac{1}{2}}\left(\hat{x}_{1}, \ldots \hat{x}_{n}\right)$, $\|\mathcal{V}\|^{2}=\|\hat{\mathcal{V}}\|^{2}=1$. Thus we have $\mathcal{A}=-2 \gamma_{s}^{2}+2 \gamma_{s} \theta_{s}, \alpha=\gamma_{s}^{2}-2 \gamma_{s} \theta_{s}+\theta_{s}^{2}-$ $R^{2}$ and

$$
\begin{aligned}
\mathcal{F}(0) & =\frac{\mathcal{A}^{2}}{4}-\alpha\left(\left\|q_{0}\right\|^{2}+\gamma_{s}^{2}\right) \\
& =\frac{\mathcal{A}^{2}}{4}-\alpha\left[\left(x_{0_{1}} \bar{x}_{0_{1}}+\cdots+x_{0_{n}} \bar{x}_{0_{n}}\right)+\gamma_{s}^{2}\right]=\hat{\mathcal{F}}(0)
\end{aligned}
$$

Also we have

$$
\mathcal{J}=\frac{\alpha}{4 \mathcal{F}(0)}\left[\left\langle q_{0}, \mathcal{V}\right\rangle+\left\langle\mathcal{V}, q_{0}\right\rangle\right]\left[\left\langle q_{0}, \hat{\mathcal{V}}\right\rangle+\left\langle\hat{\mathcal{V}}, q_{0}\right\rangle\right]
$$

and

$$
\Lambda=\frac{\alpha}{4 \mathcal{F}(0)}\left(\left[\left\langle q_{0}, \hat{\mathcal{V}}\right\rangle+\left\langle\hat{\mathcal{V}}, q_{0}\right\rangle\right]^{2}+\left[\left\langle q_{0}, \mathcal{V}\right\rangle+\left\langle\mathcal{V}, q_{0}\right\rangle\right]^{2}\right)
$$

where

$$
\left\langle q_{0}, \mathcal{V}\right\rangle+\left\langle\mathcal{V}, q_{0}\right\rangle=\left(x_{0_{1}} \bar{v}_{1}+\cdots+x_{0_{n}} \bar{v}_{n}\right)+\left(v_{1} \bar{x}_{0_{1}}+\cdots+v_{n} \bar{x}_{0_{n}}\right)
$$




$$
\left\langle q_{0}, \hat{\mathcal{V}}\right\rangle+\left\langle\hat{\mathcal{V}}, q_{0}\right\rangle=\left(x_{0_{1}} \overline{\hat{v}}_{1}+\cdots+x_{0_{n}} \overline{\hat{v}}_{n}\right)+\left(\hat{v}_{1} \bar{x}_{0_{1}}+\cdots+\hat{v}_{n} \bar{x}_{0_{n}}\right)
$$

Thus,

$$
\cos (\mathcal{X})=\left(x_{1} \bar{x}_{1}+\cdots+x_{n} \bar{x}_{n}\right)^{-\frac{1}{2}}\left(\hat{x}_{1} \overline{\hat{x}}_{1}+\cdots+\hat{x}_{n} \overline{\hat{x}}_{n}\right)^{-\frac{1}{2}}\left(x_{1} \overline{\hat{x}}_{1}+\cdots+x_{n} \overline{\hat{x}}_{n}\right)
$$

and

$$
\begin{aligned}
& \cos (\mathcal{Y}) \\
& =\frac{\left(x_{1} \bar{x}_{1}+\cdots+x_{n} \bar{x}_{n}\right)^{-\frac{1}{2}}\left(\hat{x}_{1} \overline{\hat{x}}_{1}+\cdots+\hat{x}_{n} \overline{\hat{x}}_{n}\right)^{-\frac{1}{2}}\left(x_{1} \overline{\hat{x}}_{1}+\cdots+x_{n} \overline{\hat{x}}_{n}\right)+\mathcal{J}}{\sqrt{1+\mathcal{J}^{2}+\Lambda}}
\end{aligned}
$$

with $\mathcal{J}$ and $\Lambda$ subject to (7.4.9) and (7.4.10) respectively.

Next we have

Example Take $\mathbb{H}=\mathbb{R}^{n}$ as a Hilbert space over $\mathbb{C}$ and define

$$
\langle q, \hat{q}\rangle=x_{1} \hat{x}_{1}+\cdots+x_{n} \hat{x}_{n}
$$

where $q=\left(x_{1}, \ldots, x_{n}\right)$ and $\hat{q}=\left(\hat{x}_{1}, \ldots \hat{x}_{n}\right)$. Assume $q_{0}=\left(x_{0_{1}}, \ldots, x_{0_{n}}\right)$, $\left(x_{1}^{2}+\cdots+x_{n}^{2}\right)>0$ and $\left(\hat{x}_{1}^{2}+\cdots+\hat{x}_{n}^{2}\right)>0$ then $\mathcal{V}=\left(x_{1}^{2}+\cdots+x_{n}^{2}\right)^{-\frac{1}{2}}\left(x_{1}, \ldots, x_{n}\right)$ and $\hat{\mathcal{V}}=\left(\hat{x}_{1}^{2}+\cdots+\hat{x}_{n}^{2}\right)^{-\frac{1}{2}}\left(\hat{x}_{1}, \ldots \hat{x}_{n}\right),\|\mathcal{V}\|^{2}=\|\hat{\mathcal{V}}\|^{2}=1$. Thus we have $\mathcal{A}=-2 \gamma_{s} \bar{\gamma}_{s}+\gamma_{s} \bar{\theta}_{s}+\theta_{s} \bar{\gamma}_{s}$ and $\alpha=\gamma_{s} \bar{\gamma}_{s}-\gamma_{s} \bar{\theta}_{s}-\theta_{s} \bar{\gamma}_{s}+\theta_{s} \bar{\theta}_{s}-R^{2}$ and

$$
\begin{aligned}
\mathcal{F}(0) & =\frac{\mathcal{A}^{2}}{4}-\alpha\left(\left\|q_{0}\right\|^{2}+\gamma_{s} \bar{\gamma}_{s}\right) \\
& =\frac{\mathcal{A}^{2}}{4}-\alpha\left[\left(x_{0_{1}}^{2}+\cdots+x_{0_{n}}^{2}\right)+\gamma_{s} \bar{\gamma}_{s}\right]=\hat{\mathcal{F}}(0)
\end{aligned}
$$

Also we have

$$
\begin{aligned}
\mathcal{J} & =\frac{\alpha}{4 \mathcal{F}(0)}\left[\left\langle q_{0}, \mathcal{V}\right\rangle+\left\langle\mathcal{V}, q_{0}\right\rangle\right]\left[\left\langle q_{0}, \hat{\mathcal{V}}\right\rangle+\left\langle\hat{\mathcal{V}}, q_{0}\right\rangle\right] \\
& =\frac{\alpha}{4 \mathcal{F}(0)}\left[2\left(x_{0_{1}} v_{1}+\cdots+x_{0_{n}} v_{n}\right)\right]\left[2\left(x_{0_{1}} \hat{v}_{1}+\cdots+x_{0_{n}} \hat{v}_{n}\right)\right] \\
& =\frac{\alpha}{\mathcal{F}(0)}\left[x_{0_{1}} v_{1}+\cdots+x_{0_{n}} v_{n}\right]\left[x_{0_{1}} \hat{v}_{1}+\cdots+x_{0_{n}} \hat{v}_{n}\right]
\end{aligned}
$$


and

$$
\begin{aligned}
\Lambda= & \frac{\alpha}{4 \mathcal{F}(0)}\left(\left[\left\langle q_{0}, \hat{\mathcal{V}}\right\rangle+\left\langle\hat{\mathcal{V}}, q_{0}\right\rangle\right]^{2}+\left[\left\langle q_{0}, \mathcal{V}\right\rangle+\left\langle\mathcal{V}, q_{0}\right\rangle\right]^{2}\right) \\
& =\frac{\alpha}{4 \mathcal{F}(0)}\left(\left[2\left(x_{0_{1}} \hat{v}_{1}+\cdots+x_{0_{n}} \hat{v}_{n}\right)\right]^{2}+\left[2\left(x_{0_{1}} v_{1}+\cdots+x_{0_{n}} v_{n}\right)\right]^{2}\right) \\
& =\frac{\alpha}{4 \mathcal{F}(0)} 4\left(\left[\left(x_{0_{1}} \hat{v}_{1}+\cdots+x_{0_{n}} \hat{v}_{n}\right)\right]^{2}+\left[\left(x_{0_{1}} v_{1}+\cdots+x_{0_{n}} v_{n}\right)\right]^{2}\right) \\
& =\frac{\alpha}{\mathcal{F}(0)}\left(\left[\left(x_{0_{1}} \hat{v}_{1}+\cdots+x_{0_{n}} \hat{v}_{n}\right)\right]^{2}+\left[\left(x_{0_{1}} v_{1}+\cdots+x_{0_{n}} v_{n}\right)\right]^{2}\right)
\end{aligned}
$$

Thus,

$$
\cos (\mathcal{X})=\left(x_{1}^{2}+\cdots+x_{n}^{2}\right)^{-\frac{1}{2}}\left(\hat{x}_{1}^{2}+\cdots+\hat{x}_{n}^{2}\right)^{-\frac{1}{2}}\left(x_{1} \hat{x}_{1}+\cdots+x_{n} \hat{x}_{n}\right)
$$

and

$$
\cos (\mathcal{Y})=\frac{\left(x_{1}^{2}+\cdots+x_{n}^{2}\right)^{-\frac{1}{2}}\left(\hat{x}_{1}^{2}+\cdots+\hat{x}_{n}^{2}\right)^{-\frac{1}{2}}\left(x_{1} \hat{x}_{1}+\cdots+x_{n} \hat{x}_{n}\right)+\mathcal{J}}{\sqrt{1+\mathcal{J}^{2}+\Lambda}}
$$

with $\mathcal{J}$ and $\Lambda$ subject to (7.4.11) and (7.4.12) respectively.

Next we have

Example Take $\mathbb{H}=\mathbb{R}^{n}$ as a Hilbert space over $\mathbb{R}$ and define

$$
\langle q, \hat{q}\rangle=x_{1} \hat{x}_{1}+\cdots+x_{n} \hat{x}_{n}
$$

where $q=\left(x_{1}, \ldots, x_{n}\right)$ and $\hat{q}=\left(\hat{x}_{1}, \ldots \hat{x}_{n}\right)$. Assume $q_{0}=\left(x_{0_{1}}, \ldots, x_{0_{n}}\right)$, $\left(x_{1}^{2}+\cdots+x_{n}^{2}\right)>0$ and $\left(\hat{x}_{1}^{2}+\cdots+\hat{x}_{n}^{2}\right)$ then $\mathcal{V}=\left(x_{1}^{2}+\cdots+x_{n}^{2}\right)^{-\frac{1}{2}}\left(x_{1}, \ldots, x_{n}\right)$ and $\hat{\mathcal{V}}=\left(\hat{x}_{1}^{2}+\cdots+\hat{x}_{n}^{2}\right)^{-\frac{1}{2}}\left(\hat{x}_{1}, \ldots \hat{x}_{n}\right),\|\mathcal{V}\|^{2}=\|\hat{\mathcal{V}}\|^{2}=1$. Thus we have $\mathcal{A}=-2 \gamma_{s} \bar{\gamma}_{s}+2 \gamma_{s} \theta_{s}, \alpha=\gamma_{s}^{2}-2 \gamma_{s} \theta_{s}+\theta_{s}^{2}-R^{2}$ and

$$
\begin{aligned}
\mathcal{F}(0) & =\frac{\mathcal{A}^{2}}{4}-\alpha\left(\left\|q_{0}\right\|^{2}+\gamma_{s}^{2}\right) \\
& =\frac{\mathcal{A}^{2}}{4}-\alpha\left[\left(x_{0_{1}}^{2}+\cdots+x_{0_{n}}^{2}\right)+\gamma_{s}^{2}\right]=\hat{\mathcal{F}}(0)
\end{aligned}
$$


Since $\mathbb{H}=\mathbb{R}^{n}$ over $\mathbb{R}$ then $\left\langle q_{0}, \mathcal{V}\right\rangle=\left\langle\mathcal{V}, q_{0}\right\rangle$ and $\left\langle q_{0}, \hat{\mathcal{V}}\right\rangle=\left\langle\hat{\mathcal{V}}, q_{0}\right\rangle$ therefore we have

$$
\begin{aligned}
\mathcal{J} & =\frac{\alpha}{4 \mathcal{F}(0)}\left[\left\langle q_{0}, \mathcal{V}\right\rangle+\left\langle\mathcal{V}, q_{0}\right\rangle\right]\left[\left\langle q_{0}, \hat{\mathcal{V}}\right\rangle+\left\langle\hat{\mathcal{V}}, q_{0}\right\rangle\right] \\
& =\frac{\alpha}{4 \mathcal{F}(0)}\left[2\left\langle q_{0}, \mathcal{V}\right\rangle\right]\left[2\left\langle q_{0}, \hat{\mathcal{V}}\right\rangle\right] \\
& =\frac{\alpha}{4 \mathcal{F}(0)}\left[2\left(x_{0_{1}} v_{1}+\cdots+x_{0_{n}} v_{n}\right)\right]\left[2\left(x_{0_{1}} \hat{v}_{1}+\cdots+x_{0_{n}} \hat{v}_{n}\right)\right] \\
& =\frac{\alpha}{\mathcal{F}(0)}\left[x_{0_{1}} v_{1}+\cdots+x_{0_{n}} v_{n}\right]\left[x_{0_{1}} \hat{v}_{1}+\cdots+x_{0_{n}} \hat{v}_{n}\right]
\end{aligned}
$$

and

$$
\begin{aligned}
\Lambda= & \frac{\alpha}{4 \mathcal{F}(0)}\left(\left[\left\langle q_{0}, \hat{\mathcal{V}}\right\rangle+\left\langle\hat{\mathcal{V}}, q_{0}\right\rangle\right]^{2}+\left[\left\langle q_{0}, \mathcal{V}\right\rangle+\left\langle\mathcal{V}, q_{0}\right\rangle\right]^{2}\right) \\
& =\frac{\alpha}{4 \mathcal{F}(0)}\left(\left[2\left(x_{0_{1}} \hat{v}_{1}+\cdots+x_{0_{n}} \hat{v}_{n}\right)\right]^{2}+\left[2\left(x_{0_{1}} v_{1}+\cdots+x_{0_{n}} v_{n}\right)\right]^{2}\right) \\
& =\frac{\alpha}{4 \mathcal{F}(0)} 4\left(\left[\left(x_{0_{1}} \hat{v}_{1}+\cdots+x_{0_{n}} \hat{v}_{n}\right)\right]^{2}+\left[\left(x_{0_{1}} v_{1}+\cdots+x_{0_{n}} v_{n}\right)\right]^{2}\right) \\
& =\frac{\alpha}{\mathcal{F}(0)}\left(\left[\left(x_{0_{1}} \hat{v}_{1}+\cdots+x_{0_{n}} \hat{v}_{n}\right)\right]^{2}+\left[\left(x_{0_{1}} v_{1}+\cdots+x_{0_{n}} v_{n}\right)\right]^{2}\right)
\end{aligned}
$$

Thus,

$$
\cos (\mathcal{X})=\left(x_{1}^{2}+\cdots+x_{n}^{2}\right)^{-\frac{1}{2}}\left(\hat{x}_{1}^{2}+\cdots+\hat{x}_{n}^{2}\right)^{-\frac{1}{2}}\left(x_{1} \hat{x}_{1}+\cdots+x_{n} \hat{x}_{n}\right)
$$

and

$$
\cos (\mathcal{Y})=\frac{\left(x_{1}^{2}+\cdots+x_{n}^{2}\right)^{-\frac{1}{2}}\left(\hat{x}_{1}^{2}+\cdots+\hat{x}_{n}^{2}\right)^{-\frac{1}{2}}\left(x_{1} \hat{x}_{1}+\cdots+x_{n} \hat{x}_{n}\right)+\mathcal{J}}{\sqrt{1+\mathcal{J}^{2}+\Lambda}}
$$

with $\mathcal{J}$ and $\Lambda$ subject to (7.4.13) and (7.4.14) respectively.

Next we have

Example We will show that Y.I. Gingold and H. Gingold [21] family of projections is a special case of our methodology. Take $\mathbb{H}=\mathbb{R}^{2}$ as a Hilbert space over $\mathbb{R}$ and define

$$
\langle q, \hat{q}\rangle=x \hat{x}+y \hat{y}
$$


where $q=(x, y)$ and $\hat{q}=(\hat{x}, \hat{y})$. Consider $\theta=0$ and $R=1$, and assume $q_{0}=\left(x_{0}, y_{0}\right),\left(x_{1}^{2}+x_{2}^{2}\right)>0$ and $\left(\hat{x}_{1}^{2}+\hat{x}_{2}^{2}\right)>0$ then $\mathcal{V}=\left(x_{1}^{2}+x_{2}^{2}\right)^{-\frac{1}{2}}\left(x_{1}, x_{2}\right)$ $\hat{\mathcal{V}}=\left(\hat{x}_{1}^{2}+\hat{x}_{2}^{2}\right)^{-\frac{1}{2}}\left(\hat{x}_{1}, \hat{x}_{2}\right),\|\mathcal{V}\|^{2}=\|\hat{\mathcal{V}}\|^{2}=1$. Thus we have $\mathcal{A}=-2 \gamma^{2}$, $\alpha=\gamma^{2}-1$ and

$$
\begin{aligned}
\mathcal{F}(0) & =\frac{\mathcal{A}^{2}}{4}-\alpha\left(\left\|q_{0}\right\|^{2}+\gamma_{s}^{2}\right)=\gamma_{s}^{4}-\left(\gamma_{s}^{2}-1\right)\left(\left\|q_{0}\right\|^{2}+\gamma_{s}^{2}\right) \\
& =\gamma_{s}^{4}-\left(\gamma_{s}^{2}-1\right)\left\|q_{0}\right\|^{2}-\left(\gamma_{s}^{2}-1\right) \gamma_{s}^{2}=\gamma_{s}^{4}-\left(\gamma_{s}^{2}-1\right)\left\|q_{0}\right\|^{2}-\gamma_{s}^{4}+\gamma_{s}^{2} \\
& =\gamma_{s}^{2}-\left(\gamma_{s}^{2}-1\right)\left\|q_{0}\right\|^{2}=\gamma_{s}^{2}+\left(1-\gamma_{s}^{2}\right)\left\|q_{0}\right\|^{2} \\
& =\gamma_{s}^{2}+\left(1-\gamma_{s}^{2}\right)\left(x_{0}^{2}+y_{0}^{2}\right)=\hat{\mathcal{F}}(0)
\end{aligned}
$$

Since $\mathbb{H}=\mathbb{R}^{2}$ over $\mathbb{R}$ then $\left\langle q_{0}, \mathcal{V}\right\rangle=\left\langle\mathcal{V}, q_{0}\right\rangle$ and $\left\langle q_{0}, \hat{\mathcal{V}}\right\rangle=\left\langle\hat{\mathcal{V}}, q_{0}\right\rangle$ therefore we have

$$
\begin{aligned}
\mathcal{J}= & \frac{\alpha}{4 \mathcal{F}(0)}\left[\left\langle q_{0}, \mathcal{V}\right\rangle+\left\langle\mathcal{V}, q_{0}\right\rangle\right]\left[\left\langle q_{0}, \hat{\mathcal{V}}\right\rangle+\left\langle\hat{\mathcal{V}}, q_{0}\right\rangle\right]=\frac{\alpha}{4 \mathcal{F}(0)}\left[2\left\langle q_{0}, \mathcal{V}\right\rangle\right]\left[2\left\langle q_{0}, \hat{\mathcal{V}}\right\rangle\right] \\
& =\frac{\alpha}{4 \mathcal{F}(0)} 4\left\langle q_{0}, \mathcal{V}\right\rangle\left\langle q_{0}, \hat{\mathcal{V}}\right\rangle=\frac{\alpha}{\mathcal{F}(0)}\left\langle q_{0}, \mathcal{V}\right\rangle\left\langle q_{0}, \hat{\mathcal{V}}\right\rangle \\
& =\frac{\alpha}{\mathcal{F}(0)}\left(x_{0} v_{1}+y_{0} v_{2}\right)\left(x_{0} \hat{v}_{1}+y_{0} \hat{v}_{2}\right) \\
& =\frac{-\left(1-\gamma_{s}^{2}\right)}{\gamma_{s}^{2}+\left(1-\gamma_{s}^{2}\right)\left(x_{0}^{2}+y_{0}^{2}\right)}\left(x_{0} v_{1}+y_{0} v_{2}\right)\left(x_{0} \hat{v}_{1}+y_{0} \hat{v}_{2}\right)
\end{aligned}
$$

and

$$
\begin{aligned}
\Lambda= & \frac{\alpha}{4 \mathcal{F}(0)}\left(\left[\left\langle q_{0}, \hat{\mathcal{V}}\right\rangle+\left\langle\hat{\mathcal{V}}, q_{0}\right\rangle\right]^{2}+\left[\left\langle q_{0}, \mathcal{V}\right\rangle+\left\langle\mathcal{V}, q_{0}\right\rangle\right]^{2}\right) \\
& =\frac{\alpha}{4 \mathcal{F}(0)}\left(\left[2\left\langle q_{0}, \hat{\mathcal{V}}\right\rangle\right]^{2}+\left[2\left\langle q_{0}, \mathcal{V}\right\rangle\right]^{2}\right) \\
& =\frac{\alpha}{4 \mathcal{F}(0)} 4\left(\left[\left\langle q_{0}, \hat{\mathcal{V}}\right\rangle\right]^{2}+\left[\left\langle q_{0}, \mathcal{V}\right\rangle\right]^{2}\right) \\
& =\frac{\alpha}{\mathcal{F}(0)}\left(\left[x_{0} \hat{v}_{1}+y_{0} \hat{v}_{2}\right]^{2}+\left[x_{0} v_{1}+y_{0} v_{2}\right]^{2}\right) \\
& =\frac{-\left(1-\gamma_{s}^{2}\right)}{\gamma_{s}^{2}+\left(1-\gamma_{s}^{2}\right)\left(x_{0}^{2}+y_{0}^{2}\right)}\left(\left[x_{0} \hat{v}_{1}+y_{0} \hat{v}_{2}\right]^{2}+\left[x_{0} v_{1}+y_{0} v_{2}\right]^{2}\right)
\end{aligned}
$$

Thus,

$$
\cos (\mathcal{X})=\left(x_{1}^{2}+x_{2}^{2}\right)^{-\frac{1}{2}}\left(\hat{x}_{1}^{2}+\hat{x}_{2}^{2}\right)^{-\frac{1}{2}}\left(x_{1} \hat{x}_{1}+x_{2} \hat{x}_{2}\right)
$$


and

$$
\cos (\mathcal{Y})=\frac{\left(x_{1}^{2}+x_{2}^{2}\right)^{-\frac{1}{2}}\left(\hat{x}_{1}^{2}+\hat{x}_{2}^{2}\right)^{-\frac{1}{2}}\left(x_{1} \hat{x}_{1}+x_{2} \hat{x}_{2}\right)+\mathcal{J}}{\sqrt{1+\mathcal{J}^{2}+\Lambda}}
$$

with $\mathcal{J}$ and $\Lambda$ subject to (7.4.15) and (7.4.16) respectively.

\subsection{The Angle Measure Between The Images of Two Curves in Case $q_{0}=\infty U$}

To obtain the values for $\mathcal{J}$ and $\Lambda$ given in equations (7.1.10) and (7.1.11), respectively, in the case $q_{0}=\infty U$ where we begin by observing that

$$
\begin{aligned}
\mathcal{F}(0) & =\frac{\mathcal{A}^{2}}{4}-\alpha\left(\left\|q_{0}\right\|^{2}+\gamma_{s} \bar{\gamma}_{s}\right) \\
& =-\alpha\left\|q_{0}\right\|^{2}\left(1-\frac{\frac{\mathcal{A}^{2}}{4}-\alpha \gamma_{s} \bar{\gamma}_{s}}{\alpha\left\|q_{0}\right\|^{2}}\right) \\
& \sim-\alpha\left\|q_{0}\right\|^{2} \text { as }\left\|q_{0}\right\| \longrightarrow \infty
\end{aligned}
$$

Consequently, we arrive at the natural dentition of $\mathcal{J}$ for $q_{0}=\infty U$ by observing the asymptotic behavior

$$
\begin{aligned}
\mathcal{J} & =\frac{\alpha}{4 \mathcal{F}(0)}\left[\left\langle q_{0}, \mathcal{V}\right\rangle+\left\langle\mathcal{V}, q_{0}\right\rangle\right]\left[\left\langle q_{0}, \hat{\mathcal{V}}\right\rangle+\left\langle\hat{\mathcal{V}}, q_{0}\right\rangle\right] \\
& \sim \frac{-1}{4\left\|q_{0}\right\|^{2}}\left[\left\langle q_{0}, \mathcal{V}\right\rangle+\left\langle\mathcal{V}, q_{0}\right\rangle\right]\left[\left\langle q_{0}, \hat{\mathcal{V}}\right\rangle+\left\langle\hat{\mathcal{V}}, q_{0}\right\rangle\right] \text { as }\left\|q_{0}\right\| \longrightarrow \infty \\
& =\frac{-1}{4} \frac{\left[\left\langle q_{0}, \mathcal{V}\right\rangle+\left\langle\mathcal{V}, q_{0}\right\rangle\right]}{\left\|q_{0}\right\|} \frac{\left[\left\langle q_{0}, \hat{\mathcal{V}}\right\rangle+\left\langle\hat{\mathcal{V}}, q_{0}\right\rangle\right]}{\left\|q_{0}\right\|} \text { as }\left\|q_{0}\right\| \longrightarrow \infty \\
& =\frac{-1}{4}\left[\left\langle\frac{q_{0}}{\left\|q_{0}\right\|}, \mathcal{V}\right\rangle+\left\langle\mathcal{V}, \frac{q_{0}}{\left\|q_{0}\right\|}\right\rangle\right]\left[\left\langle\frac{q_{0}}{\left\|q_{0}\right\|}, \hat{\mathcal{V}}\right\rangle+\left\langle\hat{\mathcal{V}}, \frac{q_{0}}{\left\|q_{0}\right\|}\right\rangle\right] \text { as }\left\|q_{0}\right\| \longrightarrow \infty \\
& =\frac{-1}{4}[\langle U, \mathcal{V}\rangle+\langle\mathcal{V}, U\rangle][\langle U, \hat{\mathcal{V}}\rangle+\langle\hat{\mathcal{V}}, U\rangle] \text { as }\left\|q_{0}\right\| \longrightarrow \infty
\end{aligned}
$$

Hence, we put

$$
\mathcal{J}=\frac{-1}{4}[\langle U, \mathcal{V}\rangle+\langle\mathcal{V}, U\rangle][\langle U, \hat{\mathcal{V}}\rangle+\langle\hat{\mathcal{V}}, U\rangle]
$$


The reader can easily verify that for $q_{0}=\infty U, \Lambda$ asymptotically becomes zero

$$
\begin{aligned}
& \lim _{\left\|q_{0}\right\| \rightarrow \infty} \Lambda \\
& =\lim _{\left\|q_{0}\right\| \longrightarrow \infty}\left(\frac{\alpha}{4 \mathcal{F}(0)}\right)^{2}\left(\left[\left\langle q_{0}, \hat{\mathcal{V}}\right\rangle+\left\langle\hat{\mathcal{V}}, q_{0}\right\rangle\right]^{2}+\left[\left\langle q_{0}, \mathcal{V}\right\rangle+\left\langle\mathcal{V}, q_{0}\right\rangle\right]^{2}\right) \\
& =\lim _{\left\|q_{0}\right\| \longrightarrow \infty}\left(\frac{\alpha}{4\left(\frac{\mathcal{A}^{2}}{4}-\alpha\left(\left\|q_{0}\right\|^{2}+\gamma_{s} \bar{\gamma}_{s}\right)\right)}\right)^{2} \\
& \left(\left[\left\langle q_{0}, \hat{\mathcal{V}}\right\rangle+\left\langle\hat{\mathcal{V}}, q_{0}\right\rangle\right]^{2}+\left[\left\langle q_{0}, \mathcal{V}\right\rangle+\left\langle\mathcal{V}, q_{0}\right\rangle\right]^{2}\right) \\
& =\lim _{\left\|q_{0}\right\| \longrightarrow \infty}\left(\frac{\alpha}{4\left(\frac{\mathcal{A}^{2}}{4}-\alpha\left(\left\|q_{0}\right\|^{2}+\gamma_{s} \bar{\gamma}_{s}\right)\right)}\right)^{2} \\
& q_{0}^{2}\left([\langle 1, \hat{\mathcal{V}}\rangle+\langle\hat{\mathcal{V}}, 1\rangle]^{2}+[\langle 1, \mathcal{V}\rangle+\langle\mathcal{V}, 1\rangle]^{2}\right) \\
& =\lim _{\left\|q_{0}\right\| \rightarrow \infty} \frac{q_{0}^{2}}{\left\|q_{0}\right\|^{4}}\left(\frac{\alpha}{\frac{4\left(\frac{\mathcal{A}^{2}}{4}-\alpha \gamma_{s} \bar{\gamma}_{s}\right)}{\left\|q_{0}\right\|^{2}}-\alpha}\right)^{2} \\
& \left([\langle 1, \hat{\mathcal{V}}\rangle+\langle\hat{\mathcal{V}}, 1\rangle]^{2}+[\langle 1, \mathcal{V}\rangle+\langle\mathcal{V}, 1\rangle]^{2}\right)=0
\end{aligned}
$$

\section{Glossary}

$\mathbb{B}$ be a complex Banach space.

$\mathbb{H}$ be a complex Hilbert space.

$\mathbb{R}$ be a set of real numbers.

$\mathbb{C}$ be a set of complex numbers.

$q, \hat{q}, z$ and $\hat{z} \in \mathbb{H}$.

$<,>$ be an inner product in a given complex Hilbert space $\mathbb{H}$.

$\|$ || be a norm on $\mathbb{H}$.

$d(h, \hat{h})$ a metric of a given metric space.

$c$ and $\hat{c}$ are complex numbers.

$U \neq \overrightarrow{0}, U_{1} \neq \overrightarrow{0}, U_{2} \neq \overrightarrow{0}$ and $u \neq \overrightarrow{0}$ are unit elements of $\mathbb{H}$.

$\mathbb{K}$ an one dimensional subspace namely $\mathbb{K}=\{c u: u \in \mathbb{H},\|u\|=1, c \in \mathbb{C}\}$.

$\mathbb{H}_{N}$ be an extended complex Hilbert space namely $\mathbb{H}_{N}=\mathbb{K} \times \mathbb{H}$. 
$<,>_{H_{N}}$ be a new inner product in $\mathbb{H}_{N}$.

\|\|$_{H_{N}}$ be a norm on $\mathbb{H}_{N}$

a be a real number

$Q=(\overrightarrow{0}, q), \hat{Q}=(\overrightarrow{0}, \hat{q}), Z=(c u, z), \hat{Z}=(c u, \hat{z}), O=\left(\theta_{s} u, \overrightarrow{0}\right), C=(c u, \overrightarrow{0})$ and $V=\left(\theta_{c} u, \overrightarrow{0}\right)$ are points in an extended complex Hilbert space $\mathbb{H}_{N}$.

$P$ be a fixed projection point in an extended complex Hilbert space $\mathbb{H}_{N}$

$$
p= \begin{cases}\left(\gamma_{s} u, \overrightarrow{0}\right) & \text { if a surface is a sphere } \\ \left(\gamma_{p} u, \overrightarrow{0}\right) & \text { if a surface is a paraboloid } \\ \left(\gamma_{c} u, \overrightarrow{0}\right) & \text { if a surface is a cone } \\ \left(\gamma_{h} u, \overrightarrow{0}\right) & \text { if a surface is a hyperboloid } \\ \left(\gamma_{h} u, \overrightarrow{0}\right) & \text { if a surface is a ellipsoid }\end{cases}
$$

$\mathbb{S}_{N}$ be a subset from $\mathbb{H}_{N}$ namely $\mathbb{S}_{N}=\{\overrightarrow{0}\} \times \mathbb{B}$.

$I D$ be a continuum of ideal points $I D=\{\infty U: U \in \mathbb{B}$ and $\|U\|=1\}$.

$\mathbb{U E \mathbb { B }}$ the Ultra Extended Banach space $\mathbb{B}$ namely $\mathbb{U E \mathbb { B }}=\mathbb{S}_{N} \cup I D$.

$\mathbb{U E H}$ the Ultra Extended Hilbert space $\mathbb{H}$ namely $\mathbb{U E H}=\mathbb{S}_{N} \cup I D$.

$\gamma_{g}, \gamma_{s}, \gamma_{c}, \gamma_{p}, \gamma_{h}$ and $\gamma_{e}$ are complex valued parameters.

$\theta_{s}, \theta_{c}, \theta_{p}, \theta_{h}$ and $\theta_{e}$ are complex valued parameters.

$R, r, \rho, \hat{\rho}, \rho_{1}, \rho_{2}, b$ and $d$ are positive real numbers.

$\varphi$ be a mapping form $\mathbb{H}_{N}$ to $\mathbb{R}$, namely $\varphi(Z)=r \quad Z \in \mathbb{H}_{N}$.

$\mathcal{A}=-2 \gamma_{s} \bar{\gamma}_{s}+\gamma_{s} \bar{\theta}_{s}+\theta_{s} \bar{\gamma}_{s}$.

$\alpha_{s}=\gamma_{s} \bar{\gamma}_{s}-\gamma_{s} \bar{\theta}_{s}-\theta_{s} \bar{\gamma}_{s}+\theta_{s} \bar{\theta}_{s}-R^{2}$.

$F=\frac{\mathcal{A}^{2}}{4}-\left(\|q\|^{2}+\gamma_{s} \bar{\gamma}_{s}\right) \alpha_{s}$.

$\hat{F}=\frac{\mathcal{A}^{2}}{4}-\left(\|\hat{q}\|^{2}+\gamma_{s} \bar{\gamma}_{s}\right) \alpha_{s}$.

$t_{s}=\frac{-\frac{A}{2}+\sqrt{F}}{\|q\|^{2}+\gamma_{s} \bar{\gamma}_{s}}$

$\hat{t}_{s}=\frac{-\frac{\mathcal{A}}{2}+\sqrt{\hat{F}}}{\|q\|^{2}+\gamma_{s} \bar{\gamma}_{s}}$

$\operatorname{SPM}\left(\theta_{s}, R, \gamma_{s}\right)$ is a bijection from $\mathbb{S}_{N}$ onto a subset of the sphere $\operatorname{SP}\left(\theta_{s}, R\right):=$ $\left\{Z \in \mathbb{H}_{N} \mid\|Z-O\|_{H_{N}}=R\right\}$.

$y$ be a complex number.

$L=\left(2 y-\theta_{p}\right) u$

$\mathcal{B}=2\left(\bar{\gamma}_{p}\left(\theta_{p}-y\right)+\gamma_{p} \overline{\left(\theta_{p}-y\right)}\right)$

$\alpha_{p}=-\mathcal{B}-4 y \bar{y}+2\left(y \bar{\theta}_{p}+\theta_{p} \bar{y}\right)$

$G=1-\frac{4\|q\|^{2} \alpha_{p}}{\mathcal{B}^{2}}$

$\hat{G}=1-\frac{4\|\hat{\hat{q}}\|^{2} \alpha_{p}}{\mathcal{B}^{2}}$ 


$$
\begin{aligned}
& t_{p}=\frac{-2 \alpha_{p}}{\mathcal{B}+\mathcal{B} \sqrt{G}} \\
& \hat{t}_{p}=\frac{-2 \alpha_{p}}{\mathcal{B}+\mathcal{B} \sqrt{\hat{G}}} \\
& \mathcal{N}_{0}=\frac{-2 \alpha_{p}}{\mathcal{B}+\mathcal{B} \sqrt{1-\frac{4 \rho_{1}^{2} \alpha_{p}}{\mathcal{B}^{2}}}} \\
& \tan \phi=\frac{\|\overrightarrow{C Z}\|_{H_{N}}}{\|\overrightarrow{C V}\|_{H_{N}}} \\
& m=\cot \phi \\
& \alpha_{c}=\frac{\left|\gamma_{c}\right|-\left|\theta_{c}\right|}{m} \\
& t_{c}=\frac{\alpha_{c}}{\|q\|+\frac{\left|\gamma_{c}\right|}{m}} \\
& \hat{t}_{c}=\frac{\alpha_{c}}{\|\hat{q}\|+\frac{\left|\gamma_{c}\right|}{m}} \\
& \mathcal{C}=\left(\frac{\operatorname{Re}\left(\theta_{h} \bar{\gamma}_{h}\right)}{b}\right)^{2}-\gamma_{h} \bar{\gamma}_{h} \\
& \alpha_{h}=-\mathcal{C}+\theta_{h} \bar{\theta}_{h}-b^{2} \\
& E=\mathcal{C}^{2}-\left(\|q\|^{2}-\mathcal{C}\right) \alpha_{h} \\
& \hat{E}=\mathcal{C}^{2}-\left(\|\hat{q}\|^{2}-\mathcal{C}\right) \alpha_{h} \\
& t_{h}=\frac{-\alpha_{h}}{\mathcal{C}+\sqrt{E}} \\
& \hat{t}_{h}=\frac{-\alpha_{h}}{\mathcal{C}+\sqrt{\hat{E}}} \\
& \mathcal{D}=\frac{\left(\operatorname{Re}\left(\theta_{e} \bar{\gamma}_{e}\right)\right)^{2}}{d}-\gamma_{e} \bar{\gamma}_{e} \\
& \alpha_{e}=-\mathcal{D}+\theta_{e} \bar{\theta}_{e}-d^{2} \\
& \mathcal{E}=\mathcal{D}^{2}-\left(\|q\|^{2}-\mathcal{D}\right) \alpha_{e} \\
& \hat{\mathcal{E}}=\mathcal{D}^{2}-\left(\|\hat{q}\|^{2}-\mathcal{D}\right) \alpha_{e} \\
& t_{e}=\frac{-\mathcal{D}+\sqrt{\mathcal{E}}}{\|q\|^{2}-\mathcal{D}} \\
& \hat{t}_{e}=\frac{-\mathcal{D}+\sqrt{\hat{\mathcal{E}}}}{\|q\|^{2}-\mathcal{D}}
\end{aligned}
$$

$\alpha_{g}$ is a negative real numbers.

$\mathcal{G}_{g}$ the a bijection mapping matches each point $\left(\overrightarrow{0}, q_{n}\right) \in \mathbb{U} \mathbb{E} \mathbb{H}$ with a point $Z$ on any surface

$$
\mathcal{G}_{g}\left(\left(\overrightarrow{0}, q_{n}\right)\right)= \begin{cases}\left(c_{n} u, t_{g n} q_{n}\right)=\left(\left(1-t_{g n}\right) \gamma_{g} u, t_{g n} q_{n}\right) & \text { if } q_{n} \in \mathbb{H} \\ \left(\gamma_{g} u, \sqrt{-\alpha_{g}} U\right) & \text { if } q_{n}=\infty U\end{cases}
$$

$D_{g}\{Z, \hat{Z}\}=\|Z-\hat{Z}\|_{\mathbb{H}_{N}}$ the distance between $Z$ and $\hat{Z}$ in $\mathbb{U} \mathbb{E} \mathbb{H}$.

$$
\begin{aligned}
& D_{g}^{2}\{(\overrightarrow{0}, q),(\overrightarrow{0}, \hat{q})\} \\
& =\left(\hat{t}_{g}-t_{g}\right)^{2} \gamma_{g} \bar{\gamma}_{g}+\left(t_{g}\|q\|-\hat{t}_{g}\|\hat{q}\|\right)^{2}+\hat{t}_{g} t_{g}(2\|q\|\|\hat{q}\|-\langle\hat{q}, q\rangle-\langle q, \hat{q}\rangle)
\end{aligned}
$$


$D_{g}^{2}\{(\overrightarrow{0}, q),(\overrightarrow{0}, \infty U)\}=t_{g}^{2} \gamma_{g} \bar{\gamma}_{g}+\left(t_{g}\|q\|-\sqrt{-\alpha_{g}}\right)^{2}+t_{g} \sqrt{-\alpha_{g}}(2\|q\|-\langle U, q\rangle-\langle q, U\rangle)$

$D_{g}^{2}\left\{\left(\overrightarrow{0}, \infty U_{1}\right),\left(\overrightarrow{0}, \infty U_{2}\right)\right\}=-\alpha_{g}\left\|U_{1}-U_{2}\right\|^{2}$

$D_{s}\{Z, \hat{Z}\}=\|Z-\hat{Z}\|_{H_{N}}$ the distance between $Z$ and $\hat{Z}$ in $\mathbb{U E H}$.

$\mathcal{G}_{s}\left(\left(\overrightarrow{0}, q_{n}\right)\right)$ a mapping from $\left\{\left(\overrightarrow{0}, q_{n}\right): q_{n} \in \mathbb{U} \mathbb{E} \mathbb{H}\right\}$ into the sphere

$$
\begin{aligned}
& \mathcal{G}_{s}\left(\left(\overrightarrow{0}, q_{n}\right)\right)= \begin{cases}\left(c_{n} u, t_{s n} q_{n}\right)=\left(\left(1-t_{s n}\right) \gamma_{s} u, t_{s n} q_{n}\right) & \text { if } q_{n} \in \mathbb{H} \\
\left(\gamma_{s} u, \sqrt{-\alpha_{s}} U\right) & \text { if } q_{n}=\infty U\end{cases} \\
& D_{s}^{2}\{(\overrightarrow{0}, q),(\overrightarrow{0}, \hat{q})\} \\
& =\left(\hat{t}_{s}-t_{s}\right)^{2} \gamma_{s} \bar{\gamma}_{s}+\left(t_{s}\|q\|-\hat{t}_{s}\|\hat{q}\|\right)^{2}+\hat{t}_{s} t_{s}(2\|q\|\|\hat{q}\|-\langle\hat{q}, q\rangle-\langle q, \hat{q}\rangle) \\
& D_{s}^{2}\{(\overrightarrow{0}, q),(\overrightarrow{0}, \infty U)\} \\
& =t_{s}^{2} \gamma_{s} \bar{\gamma}_{s}+\left(t_{s}\|q\|-\sqrt{-\alpha_{s}}\right)^{2}+t_{s} \sqrt{-\alpha_{s}}(2\|q\|-\langle U, q\rangle-\langle q, U\rangle)
\end{aligned}
$$

$\ell^{2}$ is the space of square summable sequences of complex number $L^{2}[0,1]$ the space of all continuous complex-valued functions on $[0,1]$ $\mathcal{L}(\mathbb{H}, \mathbb{H})$ be the set of all a linear transformation $T: \mathbb{H} \longrightarrow \mathbb{H}$ $\mathcal{G}_{p}\left(\left(\overrightarrow{0}, q_{n}\right)\right)$ the mapping from $\left\{\left(\overrightarrow{0}, q_{n}\right): q_{n} \in \mathbb{U} \mathbb{E} \mathbb{H}\right\}$ into the parabolic

$$
\mathcal{G}_{p}\left(\left(0, q_{n}\right)\right)= \begin{cases}\left(c_{n} u, t_{p n} q_{n}\right)=\left(\left(1-t_{p n}\right) \gamma_{p} u, t_{p n} q_{n}\right) & \text { if } q_{n} \in \mathbb{H} \\ \left(\gamma_{p} u, \sqrt{-\alpha_{p}} U\right) & \text { if } q_{n}=\infty U\end{cases}
$$

$D_{p}\{Z, \hat{Z}\}=\|Z-\hat{Z}\|_{H_{N}}$ the distance between $Z$ and $\hat{Z}$ in $\mathbb{U E H}$.

$$
\begin{aligned}
& D_{p}^{2}\{(\overrightarrow{0}, q),(\overrightarrow{0}, \hat{q})\} \\
& =\left(\hat{t}_{p}-t_{p}\right)^{2} \gamma_{p} \bar{\gamma}_{p}+\left(t_{p}\|q\|-\hat{t}_{p}\|\hat{q}\|\right)^{2}+\hat{t}_{p} t_{p}(2\|q\|\|\hat{q}\|-\langle\hat{q}, q\rangle-\langle q, \hat{q}\rangle) \\
& \quad D_{p}^{2}\{(\overrightarrow{0}, q),(\overrightarrow{0}, \infty U)\} \\
& \quad=t_{p}^{2} \gamma_{p} \bar{\gamma}_{p}+\left(t_{p}\|q\|-\sqrt{-\alpha_{p}}\right)^{2}+\sqrt{-\alpha_{p}}(2\|q\|-\langle U, q\rangle-\langle q, U\rangle) \\
& D_{p}^{2}\left\{\left(\overrightarrow{0}, \infty U_{1}\right),\left(\overrightarrow{0}, \infty U_{2}\right)\right\}=-\alpha_{p}\left\|U_{1}-U_{2}\right\|^{2} \\
& \mathcal{G}_{c}\left(\left(\overrightarrow{0}, q_{n}\right)\right) \text { the mapping from }\left\{\left(\overrightarrow{0}, q_{n}\right): q_{n} \in \mathbb{U E} \mathbb{H}\right\} \text { into the cone }
\end{aligned}
$$

$$
\mathcal{G}_{c}\left(\left(\overrightarrow{0}, q_{n}\right)\right)= \begin{cases}\left(c_{n} u, t_{c n} q_{n}\right)=\left(\left(1-t_{c n}\right) \gamma_{s} u, t_{c n} q_{n}\right) & \text { if } q_{n} \in \mathbb{U} \mathbb{E} \mathbb{H} \\ \left(\gamma_{c} u, \alpha_{c} U\right) & \text { if } q_{n}=\infty U\end{cases}
$$


$D_{c}\{Z, \hat{Z}\}=\|Z-\hat{Z}\|_{H_{N}}$ the distance between $Z$ and $\hat{Z}$ in $\mathbb{U E H}$.

$$
\begin{gathered}
D_{c}^{2}\{(\overrightarrow{0}, q),(\overrightarrow{0}, \hat{q})\} \\
=\left(\hat{t}_{c}-t_{c}\right)^{2} \gamma_{c} \bar{\gamma}_{c}+\left(t_{c}\|q\|-\hat{t}_{c}\|\hat{q}\|\right)^{2}+\hat{t}_{c} t_{c}(2\|q\|\|\hat{q}\|-\langle\hat{q}, q\rangle-\langle q, \hat{q}\rangle) \\
\quad D_{c}^{2}\left\{(\overrightarrow{0}, q),\left(\overrightarrow{0}, \infty U_{2}\right)\right\} \\
\quad=t_{c}^{2} \gamma_{c} \bar{\gamma}_{c}+\left(t_{c}\|q\|-\alpha_{c}\right)^{2}+\alpha_{c} t_{c}\left(2\|q\|-\left\langle U_{2}, q\right\rangle-\left\langle q, U_{2}\right\rangle\right) \\
D_{c}^{2}\left\{\left(\overrightarrow{0}, \infty U_{1}\right),\left(\overrightarrow{0}, \infty U_{2}\right)\right\}=\alpha_{c}^{2}\left\|U_{1}-U_{2}\right\|^{2}
\end{gathered}
$$

$\mathcal{G}_{h}\left(\left(\overrightarrow{0}, q_{n}\right)\right)$ the mapping from $\left\{\left(\overrightarrow{0}, q_{n}\right): q_{n} \in \mathbb{U} \mathbb{E} \mathbb{H}\right\}$ into the hyperboloid

$$
\mathcal{G}_{h}\left(\left(\overrightarrow{0}, q_{n}\right)\right)= \begin{cases}\left(c_{n} u, t_{h n} q_{n}\right)=\left(\left(1-t_{h n}\right) \gamma_{h} u, t_{h n} q_{n}\right) & \text { if } q_{n} \in \mathbb{H} \\ \left(\gamma_{p} u, \sqrt{-\alpha_{h}} U\right) & \text { if } q_{n}=\infty U\end{cases}
$$

$D_{h}\{Z, \hat{Z}\}=\|Z-\hat{Z}\|_{\mathbb{H}_{N}}$ the distance between $Z$ and $\hat{Z}$ in $\mathbb{U E} \mathbb{H}$.

$$
\begin{aligned}
& \quad D_{h}^{2}\{(\overrightarrow{0}, q),(\overrightarrow{0}, \hat{q})\} \\
& =\left(\hat{t}_{h}-t_{h}\right)^{2} \gamma_{h} \bar{\gamma}_{h}+\left(t_{h}\|q\|-\hat{t}_{h}\|\hat{q}\|\right)^{2}+\hat{t}_{h} t_{h}(2\|q\|\|\hat{q}\|-\langle\hat{q}, q\rangle-\langle q, \hat{q}\rangle) \\
& \quad D_{h}^{2}\{(\overrightarrow{0}, q),(\overrightarrow{0}, \infty U)\} \\
& \quad=t_{h}^{2} \gamma_{h} \bar{\gamma}_{h}+\left(t_{h}\|q\|-\sqrt{-\alpha_{h}}\right)^{2}+\sqrt{-\alpha_{h}}(2\|q\|-\langle U, q\rangle-\langle q, U\rangle) \\
& D_{h}^{2}\left\{\left(\overrightarrow{0}, \infty U_{1}\right),\left(\overrightarrow{0}, \infty U_{2}\right)\right\}=-\alpha_{h}\left\|U_{1}-U_{2}\right\|^{2} \\
& \mathcal{G}_{e}\left(\left(\overrightarrow{0}, q_{n}\right)\right) \text { the mapping from }\left\{\left(\overrightarrow{0}, q_{n}\right): q_{n} \in \mathbb{U E} \mathbb{H}\right\} \text { into the ellipsoid }
\end{aligned}
$$

$$
\mathcal{G}_{e}\left(\left(\overrightarrow{0}, q_{n}\right)\right)= \begin{cases}\left(c_{n} u, t_{e n} q_{n}\right)=\left(\left(1-t_{e n}\right) \gamma_{e} u, t_{e n} q_{n}\right) & \text { if } q_{n} \in \mathbb{H} \\ \left(\gamma_{e} u, \sqrt{-\alpha_{e}} U\right) & \text { if } q_{n}=\infty U\end{cases}
$$

$D_{e}\{Z, \hat{Z}\}=\|Z-\hat{Z}\|_{\mathbb{H}_{N}}$ the distance between $Z$ and $\hat{Z}$ in $\mathbb{U E H}$.

$$
\begin{aligned}
& D_{e}^{2}\{(\overrightarrow{0}, q),(\overrightarrow{0}, \hat{q})\} \\
& =\left(\hat{t}_{e}-t_{e}\right)^{2} \gamma_{e} \bar{\gamma}_{e}+\left(t_{e}\|q\|-\hat{t}_{e}\|\hat{q}\|\right)^{2}+\hat{t}_{e} t_{e}(2\|q\|\|\hat{q}\|-\langle\hat{q}, q\rangle-\langle q, \hat{q}\rangle)
\end{aligned}
$$




$$
\begin{aligned}
& \quad D_{e}^{2}\left\{(\overrightarrow{0}, q),\left(\overrightarrow{0}, \infty U_{2}\right)\right\} \\
& \quad=t_{e}^{2} \gamma_{e} \bar{\gamma}_{e}+\left(t_{e}\|q\|-\sqrt{-\alpha_{e}}\right)^{2}+t_{e} \sqrt{-\alpha_{e}}\left(2\|q\|-\left\langle U_{2}, q\right\rangle-\left\langle q, U_{2}\right\rangle\right) \\
& D_{e}^{2}\left\{\left(\overrightarrow{0}, \infty U_{1}\right),\left(\overrightarrow{0}, \infty U_{2}\right)\right\}=-\alpha_{e}\left\|U_{1}-U_{2}\right\|^{2} \\
& \mathcal{V}, \hat{\mathcal{V}} \text { be two unit vectors in a given complex Hilbert space } \mathbb{H} . \\
& \mathcal{X} \text { be a angle between any two arrows representing } \mathcal{V}, \hat{\mathcal{V}} . \\
& \mathcal{Y} \text { be an image of } \mathcal{X} \text { under the projection on the sphere. }
\end{aligned}
$$




\section{Bibliography}

[1] M. Agrawala, D. Zorin, and T. Munzner. Artistic multiprojection rendering. In Rendering Techniques 2000, pp. 125-136. Springer, Vienna, 2000 .

[2] L. V. Ahlfors, Complex Analysis, An Introduction To The Theory Of Analytic Functions Of One Complex Variable, Third Edition, McGrawHill Inc, 1979.

[3] K. Andersen, The Geometry Of An Art: The History Of The Mathematical Theory Of Perspective From Alberti To Monge. Springer, 2007.

[4] A. A. Andronov, E. A. Leontovich, I. I. Gordon, and A. G. Maier, Qualitative Theory Of Second-Order Dynamic Systems, John Wiley and Sons 1973.

[5] S. Axler Linear algebra Done Right. Third Edition. Springer International Publishing 2015.

[6] V. Balestro, H. Martini, and R. Teixeira, Geometric Constants For Quantifying The Difference Between Orthogonality, Annals of Functional Analysis, Vol. 7, NO. 4 (2016), 656-671. https://projecteuclid.org/euclid.afa/1475685112

[7] V. Balestro, H. Martini, and R. Teixeira. Geometric Properties Of A Sine Function Extendable To Arbitrary Normed Planes, Monatshefte für Mathematik, (2017), Vol. 182 Issue 4, 781-800.

https://arxiv.org/pdf/1601.06287.pdf

[8] I. Bendixon, Sur les courbes définies par des équations differérentielles, Acta Mathematica, Vol. 24, No. 1, (1901), pp. 1-88.

doi:10.1007/BF02403068 
[9] D. Bouwmeester, A. Ekert and A. Zeilinger, (Eds.), The Physics of Quantum Information, Spring-Verlag, Berlin Heidelberg,2000.

[10] R. Carroll, M. Agrawala, and A. Agarwala, Optimizing contentpreserving projections for wide-angle images. ACM Trans. Graph., 28(3), pp.43-1. 2009.

[11] C. Chicone and J. Sotomayor, On A Class Of Complete Polynomial Vector Fields In The Plane, Journal of Differential Equations Vol. 61, No. 3 (1986), 398-418. doi:10.1016/0022-0396(86)90113-0

[12] A. Cima and J. Llibre, Bounded Polynomial Vector Fields, Transactions of the American Mathematical Society, Vol. 318. No. 2. (1990), 557-579. doi:10.1090/S0002-9947-1990-0998352-5

[13] H. Damisch, The Origin Of Perspective, Massachusetts Institute Of Technology, 1994.

[14] C. F. Dunkl and Yuan Xu. Orthogonal Polynomials of Several Variables.Cambridge University Press 2001.

[15] U. Elias and H. Gingold, Critical Points At Infinity And Blow Up Of Solutions Of Autonomous Polynomial Differential Systems Via Compactification, Journal of Mathematical Analysis and Applications, Vol. 318, No. 1, 2006, pp. 305-322. https://doi.org/10.1016/j.jmaa.2005.06.002

[16] G. Freud. Orthogonal Polynomials. Pergamon Press(Aust.) 2011.

[17] A. Galantai and Cs. J. Hegedus, Jordan's Principal Angles In Complex Vector Spaces Numerical linear Algebra With Applications. 13 (2006), 589-598. DOI: $10.1002 /$ nla.491

[18] W. Gautschi. Orthogonal Polynomials Computation and Approximation. Oxford University Press 2004.

[19] H. Gingold, Compactification Applied To A discrete Competing System Model, International Journal of Pure and Applied Mathematics Vol. 74 No. 2 (2012), 147-170

[20] H. Gingold, Divergence Of Solutions Of Polynomials Finite Difference Equations, Proceedings A of the Royal Society of Edinburgh, Vol. 142, No. 4, (2012), pp. 787-804. doi:10.1017/S0308210510000077 
[21] H. Gingold, Compactification And Divergence Of Solutions Of Polynomial Finite Difference Systems Of Equations, Dynamics Of Continuous, Discrete and Impulsive Systems Series B: Applied Algorithms, Vol. 18, No. 3, (2011), pp. 315-335

[22] H. Gingold and D. Solomon, On Completeness Of Quadratic Systems, Nonlinear Analysis, Vol. 74, No. 12, (2011), pp. 4234-4240.

[23] H. Gingold and D. Solomon, The Lorenz System Has A global Repeller At Infinity, Journal of Nonlinear Mathematical Physics, Vol. 18, No. 2, (2011), 183-189. https://doi.org/10.1142/S1402925111001489

[24] H. Gingold, Approximation Of Unbounded Functions Via Compactification, Journal of Approximation Theory, Vol.131 No. 2 (2004), 284-305. https://doi.org/10.1016/j.jat.2004.08.001

[25] H. Gingold, Y. Gingold and S. Hamad. A spherical projection of a complex Hilbert space is conformal iff it is the stereographic projection. Differential Geometry - Dynamical Systems, Vol.20, 2018, pp. 38-71.

[26] Y.I. Gingold and H. Gingold, Geometrical Properties Of A Family Of Compactifications. Balkan Journal of Geometry and Its Applications, Vol.12, No.1, (2007), pp. 44-55.

[27] Y. I. Gingold and H. Gingold, Simulation Of Perspective By Nonlinear Transformations, Mathematical Modelling and Analysis, Vol. 18 Issue 3 , (2013), PP 346-357. https://doi.org/10.3846/13926292.2013.804887

[28] K. T. Hahn, Trigonometry On The Unit Ball of a Complex H Space Bulletin of The American mathematical Society Vol. 81, No. 1, 1975.

[29] S. T. Heath A history Of Greek Mathematics, Vol.II, Oxford at the Clarendon Press, 1921.

[30] J. Hell, Conley Index At Infinity, der Freien Universitat Berlin 2010 Tag der Disputation: 9. November 2009.

[31] E. Hille, Analytic Function Theory, Volumes 1, 2, Chelsea Publishing Company, New York, 1982.

[32] D. Hockney. Secret knowledge: Rediscovering the lost techniques of the old masters. Penguin Putnam, 2001. 
[33] D.W. Jordan and P. Smith, Nonlinear Ordinary Differential Equations An Introduction For Scientists And Engineers, 4th edition, Oxford University Press Inc., New York, 2007.

[34] E. Kasner and J. De Cicco, Converse of Ptolemy's Theorem On Stereographic Projection, Proceedings of the National Academy of Sciences of the United States of America, Vol. 31, No. 10 (1945), pp. 338-342

[35] E. Kreyszig, Introductory Functional Analysis With Applications, John Wiley and Sons. Inc. 1978.

[36] Y. Kubo, Z. Jie and K. Hirota, A method For Transformation Of 3D Space Into Ukiyo-e composition , ACM SIGGRAPH Asia Art Paper, (2008) PP 29-35. https://doi.org/10.1145/1504229.1504250

[37] J-w. Lee, C. H. Kim and E. K. Lee, J. Kim and S. Lee, Qubit Geometry And Conformal Mapping, Quantum Information Processing Vol. 1, No. 1/2, (2002), 129-134. https://arxiv.org/pdf/quant-ph/0201014.

[38] S. Lefschetz, Differential Equations: Geometric Theory, Dover, New York, 1977.

[39] H. Martini, On Anglar Bisectors In Normed Linear Spaces, Noti di Mathematica Vol. 30, No. 1 , (2010), PP 107-109 doi:10.1285/i15900932v30n1p107

[40] James R. Munkres Topology second edition Prentice Hall Ine (2000).

[41] G. Najarbashi ,B. Seifi, and S. Mirzaei, Two and Three-Qubits Geometry, Quaternionic and Octonionic Conformal Maps, and Intertwining Stereographic Projection July/21/ 2018 arXiv:1501.06013v2 [quant-ph] 12 Nov 2015

[42] G. Najarbashi and B. Seifi, Relation Between Stereographic Projection and Concurrence Measure in Bipartite Pure States July/ 9/ 2018 arXiv:1512.07006v2 [quant-ph] 16 Jun 2016

[43] C. W. O'Hara and D. R. Ward, An Introduction To Projective Geometry, Oxford Clarendon Press, 1937.

[44] F. W. J. Olver Introduction to Asymptotics and Special Functions. Academic Press, INC, (1974). 
[45] L. Perko, Differential Equations And Dynamical Systems, 3rd edition, Springer-Verlag, New York. Inc., 2001.

[46] D. Peterson. How to Turn a Smartphone Panorama Into a 'Tiny Planet' Photo. urlhttps://petapixel.com/2013/08/17/how-to-turn-asmartphone-panorama-into-a-tiny-planet-photo/. August 17, 2013. Accessed December 20, 2017.

[47] H. Poincaré, Memoire sur les courbes definies par une equation differentielle, Jour. de Math. pure et appl., (1881), 375-422.

[48] R. T. Rockafellar. Convex Analysis. Princeton University Press 1970.

[49] K. A. Ross Elementary Analysis the Theory of Calculus. 1980 Springer Science+Business Media, Inc.

[50] H. L. Royden and P. M. Fitzpatrick, Real Analysis Fourth Edition , Pearson Education, Inc. 2010.

[51] G. Sansone and R. Conti, Non-linear Differential Equations, Pergamon Press. Oxford, 1964,

[52] K. Scharnhorst. Angles In Complex Vector Spaces. Acta Applicandae Mathematica, Vol. 69, Issue 1,(2001), 95-103.

DOI: $10.1023 / \mathrm{A}: 1012692601098$

[53] B. Smus. Three approaches to VR lens distortion. 2016. Accessed 201212-20. urlhttp://smus.com/vr-lens-distortion/

[54] A. Takayasu, K. Matsue, T. Sasaki, K. Tanaka, M. Mizuguchi and S. Oishi, Numerical Validation of Blow-up Solutions Of Ordinary Differential Equations, Journal of Computational and Applied Mathematics Vol. 314, (2017), PP 10-29. https://doi.org/10.1016/j.cam.2016.10.013

[55] V. W. Thurey. The Complex Angle In Normed Spaces Revue Roumaine de Mathématiques Pures et Appliqués Vol. 60 No. 2 (2015) PP 177-197. doi:10.1088/1742-6596/490/1/012038

[56] W. F. Trench Introduction to Real Analysis Free Edition 1.07, September, 2011. This book was published previously by pearson education. 
[57] J. E. Valentine and S. G. Wayment, Wilson Angles In Linear Normed Spaces. Pacific journal of mathematics Vol. 36, No. 1, 1971.

[58] Vishwanath, D., Girshick, A.R. and Banks, M.S., Why pictures look right when viewed from the wrong place. Nature neuroscience, $8(10)$, pp.1401-1410. 2005.

[59] Wikipedia contributors. Distortion (optics). Wikipedia, The Free Encyclopedia, 31 Oct. 2017. Web. 20 Dec. 2017.

[60] M. Zannoli and M. S. Banks. The Perceptual Consequences of Curved Screens. ACM Trans. Appl. Percept. 15, 1, Article 6. 2017. DOI: https://doi.org/10.1145/3106012

[61] D. Zorin and A. H. Barr, Correction of Geometric Perceptual Distortions In Pictures, the ACM SIGGRAPH Conference on Computer Graphics Publisher (1995) 257-264. 